\title{
Mono- and Bis-Cyclometalated Palladium Complexes: Synthesis, Characterization and Catalytic Activity
}

\author{
Sebastian Molitor, Christopher Schwarz, Viktoria H. Gessner \\ Institut für Anorganische Chemie, Julius-Maximilians-Universität Würzburg, Am Hubland, \\ 97074 Würzburg, Germany.
}

\section{Index}

1. Experimental Details $\quad$ S2

2. NMR spectra of the isolated compounds S5

3. Crystal Structure Determination $\quad$ S47

3.1 Crystal Structure Determination of 1a S52

3.2 Crystal Structure Determination of $\mathbf{1 b} \quad$ S54

3.3 Crystal Structure Determination of 1d $\quad$ S57

3.4 Crystal Structure Determination of 1e S59

3.5 Crystal Structure Determination of $\mathbf{1 f} \quad$ S61

3.6 Crystal Structure Determination of 2a $\quad$ S64

3.7 Crystal Structure Determination of $\mathbf{2 b} \quad$ S68

3.8 Crystal Structure Determination of 2c S72

3.9 Crystal Structure Determination of 2d $\quad$ S75

3.10 Crystal Structure Determination of 2e S78

3.11 Crystal Structure Determination of $2 \mathbf{f} \quad$ S81

3.12 Crystal Structure Determination of $\mathbf{3}$ S85

3.13 Crystal Structure Determination of $5 \quad$ S91

4. Transition states for the formation of $6 \quad$ S94

5. HRMS (ESI, APCI) of complexes 2a-6 S95

6. References $\quad$ S100 


\section{Experimental Details}

General Methods. All experiments were carried out under a dry, oxygen-free argon atmosphere using standard Schlenk techniques. Involved solvents were dried over sodium or potassium and distilled prior to use. $\mathrm{H}_{2} \mathrm{O}$ is distilled water. ${ }^{1} \mathrm{H},{ }^{13} \mathrm{C}\left\{{ }^{1} \mathrm{H}\right\},{ }^{29} \mathrm{Si}\left\{{ }^{1} \mathrm{H}\right\}$ and ${ }^{31} \mathrm{P}\left\{{ }^{1} \mathrm{H}\right\}$ NMR spectra were recorded on Avance-500,0 Avance- 400 or Avance-300 spectrometers at $22{ }^{\circ} \mathrm{C}$ if not stated otherwise. All values of the chemical shift are in ppm regarding the $\delta$-scale. All spin-spin coupling constants $(\mathrm{J})$ are printed in Hertz $(\mathrm{Hz})$. To display multiplicities and signal forms correctly the following abbreviations were used: $\mathrm{s}=$ singlet, $\mathrm{d}=$ doublet, $\mathrm{t}=$ triplet, $\mathrm{m}=$ multiplet, $\mathrm{br}=$ broad signal. Signal assignment was supported by DEPT and HMQC experiments. Elemental analyses were performed on an Elementarvario MICRO-cube elemental analyzer. High-resolution mass spectra (ESI, APCI) were recorded on an Exactive $^{\mathrm{TM}}$ Plus Orbitrap Mass Spectrometer from Thermo Scientific. For a better ionization in the cases of $\mathbf{2 c}, \mathbf{2 d}$ and $\mathbf{6}$ acetonitrile was added to the solutions of the complexes. All reagents were purchased from Sigma-Aldrich, ABCR, Rockwood Lithium or Acros Organics and used without further purification. The compounds $1 \mathbf{a}$ and $\mathbf{1 c}$ and their protonated precursors have been prepared according to literature procedures. ${ }^{[1]}$

Synthesis of diprotonated precursor of 1e. Diphenylmethylphosphine sulfide ( $705 \mathrm{mg}, 3.03 \mathrm{mmol}$ )

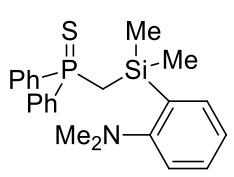
was dissolved in THF $(10 \mathrm{~mL})$ and cooled to $-78^{\circ} \mathrm{C}$. At this temperature 1.2 equivalents of $n \mathrm{BuLi}(2.35 \mathrm{~mL}, 3.64 \mathrm{mmol}, 1.55 \mathrm{M}$ in hexane) were added and the resultant yellow mixture stirred for $18 \mathrm{~h}$ at low temperature. In a second Schlenk flask, 1.2 equivalents of $o$-(Chlorodimethylsilyl)- $N, N$-dimethylaniline (779 $\mathrm{mg}, 3.64$ $\mathrm{mmol}$ ) were dissolved in THF (35 $\mathrm{mL})$ and cooled to $-78{ }^{\circ} \mathrm{C}$. The lithiated phosphine sulfide was added dropwise by cannula transfer and slowly warmed to ambient temperature after completion of the addition. After stirring for $18 \mathrm{~h}$, distilled water $(25 \mathrm{~mL})$ was added and the mixture extracted with diethyl ether. Drying over sodium sulfate and removal of the solvent afforded a yellowish oil, which was purified by flash chromatography on silica gel (diethyl ether/pentane $=1: 3$ ) giving the product as colorless oil (800 mg, $1.95 \mathrm{mmol}, 65 \%)$. ${ }^{1} \mathrm{H} \mathrm{NMR}\left(\mathrm{CDCl}_{3}, 400.1 \mathrm{MHz}\right): \delta=0.33$ (s, $\left.6 \mathrm{H} ; \mathrm{Si}\left(\mathrm{CH}_{3}\right)_{2}\right)$, $2.41\left(\mathrm{~s}, 6 \mathrm{H} ; \mathrm{N}\left(\mathrm{CH}_{3}\right)_{2}\right), 7.17\left(\mathrm{td},{ }^{3} J_{\mathrm{HH}}=7.3 \mathrm{~Hz},{ }^{4} J_{\mathrm{HH}}=1.2 \mathrm{~Hz}, 1 \mathrm{H} ; \mathrm{C} H_{\text {Aniline }}\right), 7.31\left(\mathrm{~m}, 1 \mathrm{H} ; \mathrm{CH}_{\text {Aniline }}\right)$,

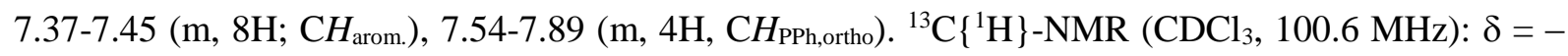
$0.2\left(\mathrm{Si}\left(\mathrm{CH}_{3}\right)_{2}\right), 20.0\left(\mathrm{~d},{ }^{1} J_{\mathrm{CP}}=46.3 \mathrm{~Hz} ; \mathrm{CH}_{2}\right), 47.3\left(\mathrm{~N}\left(\mathrm{CH}_{3}\right)_{2}\right), 122.1\left(\mathrm{CH}_{\text {Aniline }}\right), 125.5\left(\mathrm{CH}_{\text {Aniline }}\right), 128.4$ $\left(\mathrm{d},{ }^{3} J_{\mathrm{CP}}=12.1 \mathrm{~Hz} ; C \mathrm{H}_{\mathrm{PPh}, \mathrm{meta}}\right), 130.8\left(\mathrm{~d},{ }^{2} J_{\mathrm{CP}}=10.5 \mathrm{~Hz} ; C \mathrm{H}_{\mathrm{PPh}, \text { ortho }}\right), 130.9$ (d, ${ }^{4} J_{\mathrm{CP}}=2.9 \mathrm{~Hz} ; C_{\mathrm{PPh}, \text { para }}$ ), $131.0\left(\mathrm{CH}_{\text {Aniline }}\right), 135.4\left(\mathrm{CH}_{\text {Aniline }}\right), 136.3\left(\mathrm{~d},{ }^{1} J_{\mathrm{CP}}=88.4 \mathrm{~Hz} ; C_{\mathrm{PPh}, \mathrm{pss}}\right), 136.8\left(\mathrm{~d},{ }^{3} J_{\mathrm{CP}}=6.6 \mathrm{~Hz} ; C_{\text {Aniline }}\right)$, $160.8\left(\mathrm{COCH}_{3 \text { Aniline }}\right) .{ }^{29} \mathrm{Si}\left\{{ }^{1} \mathrm{H}\right\} \mathrm{NMR}\left(\mathrm{CDCl}_{3}, 59.6 \mathrm{MHz}\right): \delta=-6.3(\mathrm{br}) .{ }^{31} \mathrm{P}\left\{{ }^{1} \mathrm{H}\right\} \mathrm{NMR}\left(\mathrm{CDCl}_{3}, 162.0\right.$ $\mathrm{MHz}): \delta=38.7$.

Synthesis of diprotonated precursor of 1f. Diphenylmethylphosphine sulfide (705 mg, $3.03 \mathrm{mmol}$ )

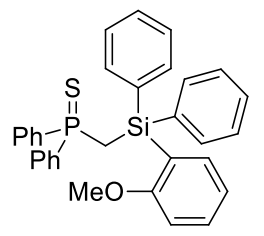
was dissolved in THF $(10 \mathrm{~mL})$ and cooled to $-78{ }^{\circ} \mathrm{C}$. At this temperature 1.1 equivalents of $n \mathrm{BuLi}(2.15 \mathrm{~mL}, 3.34 \mathrm{mmol}, 1.55 \mathrm{M}$ in hexane) were added and the resultant yellow mixture stirred for $18 \mathrm{~h}$ at low temperature. In a second Schlenk flask, 1.2 equivalents of $o$-(Chlorodiphenylsilyl)anisole $(1.08 \mathrm{~g}, 3.34 \mathrm{mmol})$ were dissolved in THF $(10 \mathrm{~mL})$ and cooled to $-78{ }^{\circ} \mathrm{C}$. The lithiated phosphine sulfide was added dropwise by cannula transfer and slowly warmed to ambient temperature after completion of the addition. After stirring for $18 \mathrm{~h}$, distilled water $(20 \mathrm{~mL})$ was added and the mixture extracted with diethyl ether. Drying over sodium sulfate and removal of the solvent afforded a yellowish solid, which was purified by flash chromatography on silica gel (dichloromethane/pentane $=1: 2$ ) giving the 
product as colorless solid (928 mg, $1.78 \mathrm{mmol}, 59 \%) .{ }^{1} \mathrm{H} \mathrm{NMR}\left(\mathrm{CDCl}_{3}, 400.1 \mathrm{MHz}\right): \delta=3.00\left(\mathrm{~d},{ }^{2} J_{\mathrm{HP}}\right.$ $\left.=16.1 \mathrm{~Hz}, 2 \mathrm{H} ; \mathrm{CH}_{2}\right), 3.58\left(\mathrm{~s}, 3 \mathrm{H} ; \mathrm{OCH}_{3}\right), 6.78\left(\mathrm{br} \mathrm{d},{ }^{3} J_{\mathrm{HH}}=8.2 \mathrm{~Hz} ; 1 \mathrm{H} ; \mathrm{CH}_{\text {Anisole }}\right), 6.90\left(\mathrm{td},{ }^{3} J_{\mathrm{HH}}=7.3\right.$ $\left.\mathrm{Hz},{ }^{5} J_{\mathrm{HH}}=0.8 \mathrm{~Hz}, 1 \mathrm{H} ; \mathrm{C} H_{\text {Anisole }}\right), 7.18-7.34\left(\mathrm{~m}, 13 \mathrm{H} ; \mathrm{CH}_{\text {arom. }}\right), 7.39\left(\mathrm{ddd},{ }^{3} J_{\mathrm{HH}}=8.4 \mathrm{~Hz},{ }^{3} J_{\mathrm{HH}}=7.4\right.$ $\left.\mathrm{Hz},{ }^{4} J_{\mathrm{HH}}=1.8 \mathrm{~Hz}, 1 \mathrm{H} ; \mathrm{CH}_{\text {Anisole }}\right), 7.53-7.55\left(\mathrm{~m}, 4 \mathrm{H} ; \mathrm{CH}_{\mathrm{SiPh}, \text { ortho }}\right), 7.66-7.72\left(\mathrm{~m}, 4 \mathrm{H} ; \mathrm{CH}_{\mathrm{PPh}, \text { ortho }}\right)$. ${ }^{13} \mathrm{C}\left\{{ }^{1} \mathrm{H}\right\}$ NMR $\left(\mathrm{CDCl}_{3}, 100.6 \mathrm{MHz}\right): \delta=18.8\left(\mathrm{~d},{ }^{1} J_{\mathrm{CP}}=48.7 \mathrm{~Hz} ; \mathrm{CH}_{2}\right), 54.9\left(\mathrm{OCH}_{3}\right), 110.1\left(\mathrm{CH}_{\text {Anisole }}\right)$, $120.8\left(\mathrm{CH}_{\text {Anisole }}\right), 122.3\left(\mathrm{~d},{ }^{3} J_{\mathrm{CP}}=4.1 \mathrm{~Hz} ; C_{\text {Anisole,ipso }}\right), 127.4\left(C_{\mathrm{S}} \mathrm{H}_{\mathrm{SPh}, \text { meta }}\right), 128.2\left(\mathrm{~d},{ }^{3} J_{\mathrm{CP}}=12.2 \mathrm{~Hz}\right.$; $\left.C_{\mathrm{PPh}, \text { meta }}\right), 129.3$ ( $\left.\mathrm{CH}_{\mathrm{SiPh}, \text { para }}\right), 130.6$ (d, $\left.{ }^{4} J_{\mathrm{CP}}=2.9 \mathrm{~Hz} ; C_{\mathrm{PPh}, \text { para }}\right), 130.8$ (d, ${ }^{2} J_{\mathrm{CP}}=10.5 \mathrm{~Hz} ; C_{\mathrm{PPh}, \text { ortho }}$ ), $132.0\left(\mathrm{CH}_{\text {Anisole }}\right), 133.8\left(\mathrm{~d},{ }^{3} J_{\mathrm{CP}}=2.3 \mathrm{~Hz} ; C_{\mathrm{SiPh}, \mathrm{psso}}\right), 135.9\left(\mathrm{~d},{ }^{1} J_{\mathrm{CP}}=81.2 \mathrm{~Hz} ; C_{\mathrm{PPh}, \mathrm{psso}}\right), 136.3$ $\left(\mathrm{CH}_{\text {SiPh,ortho }}\right), 138.1\left(\mathrm{CH}_{\text {Anisole }}\right), 164.3\left(\mathrm{COCH}_{3 \text { Anisole }}\right) .{ }^{29} \mathrm{Si}\left\{{ }^{1} \mathrm{H}\right\} \mathrm{NMR}\left(\mathrm{CDCl}_{3}, 79.5 \mathrm{MHz}\right): \delta=-14.6(\mathrm{~d}$, $\left.{ }^{2} J_{\mathrm{SiP}}=4.2 \mathrm{~Hz}\right) .{ }^{31} \mathrm{P}\left\{{ }^{1} \mathrm{H}\right\} \mathrm{NMR}\left(\mathrm{CDCl}_{3}, 162.0 \mathrm{MHz}\right): \delta=38.1$. Anal. Calc. for $\mathrm{C}_{32} \mathrm{H}_{29} \mathrm{OPSSi}: \mathrm{C}, 73.81$; H, 5.61; S, 6.16. Found: C, 73.61; H, 5.75; S, 6.09.

\section{General procedure for the preparation of the halogenated precursors 1.}

The corresponding phosphine sulfide was dissolved in THF and cooled to $-78{ }^{\circ} \mathrm{C}$. $n \mathrm{BuLi}$ (1.1 eq., 1.55 $\mathrm{M}$ in hexane) was added and the yellow solution was stirred for $1 \mathrm{~h}$ at low reaction temperatures and additionally $17 \mathrm{~h}$ at room temperature. In a second Schlenk flask, hexachloroethane or elemental iodine (1.1 eq.) was dissolved in THF and cooled to $-78^{\circ} \mathrm{C}$. The lithiated phosphine sulfide was slowly added by cannula transfer and after complete addition warmed to room temperature. The yellow mixture was stirred for $18 \mathrm{~h}$ at room temperature and then quenched by addition of distilled water $(20 \mathrm{~mL})$. Extraction with diethyl ether $(3 \times 50 \mathrm{~mL})$, drying over sodium sulfate and removal of the solvent afforded the crude products as yellow solids. The solids were purified by flash chromatography on silica gel (dichloromethane/pentane $=1: 1$ or 1:2) giving the halogenated products as colorless solids.

Synthesis of $\mathbf{1 b}$. This complex was synthesized according to the general procedure giving the product

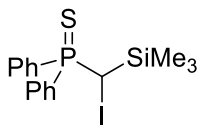
as a colorless solid $(300 \mathrm{mg}, 486 \mu \mathrm{mol}, 25 \%) .{ }^{1} \mathrm{H}$ NMR $\left(\mathrm{CDCl}_{3}, 500.1 \mathrm{MHz}\right): \delta=4.84$ $\left(\mathrm{d},{ }^{2} J_{\mathrm{HP}}=10.5 \mathrm{~Hz}, 1 \mathrm{H} ; \mathrm{CHI}\right), 7.07-7.11\left(\mathrm{~m}, 2 \mathrm{H} ; \mathrm{C} H_{\mathrm{PPh}, \text { meta }}\right), 7.20-7.26(\mathrm{~m}, 7 \mathrm{H}$; $\mathrm{CH}_{\text {SiPh,meta }}$ and $\left.\mathrm{CH}_{\mathrm{PPh}, \text { para }}\right), 7.31-7.35\left(\mathrm{~m}, 3 \mathrm{H} ; \mathrm{CH}_{\mathrm{SiPh}, \text { para }}\right), 7.38-7.43\left(\mathrm{~m}, 3 \mathrm{H}, \mathrm{C} H_{\mathrm{PPh} \text {,meta }}\right.$ and $\left.\mathrm{CH}_{\text {PPh,para }}\right), 7.65-7.70\left(\mathrm{~m}, 8 \mathrm{H}, \mathrm{C} H_{\mathrm{PPh}, \text { ortho }}\right.$ and $\left.\left.\mathrm{CH}_{\mathrm{SiPh}, \text { ortho }}\right), 7.94-7.98\left(\mathrm{~m}, 2 \mathrm{H} ; \mathrm{C} H_{\mathrm{PPh}, \text { ortho }}\right){ }^{13} \mathrm{C}^{1}{ }^{1} \mathrm{H}\right\}$ $\operatorname{NMR}\left(\mathrm{CDCl}_{3}, 125.8 \mathrm{MHz}\right): \delta=7.8\left(\mathrm{~d},{ }^{1} J_{\mathrm{CP}}=33.9 \mathrm{~Hz} ; \mathrm{CHCl}\right), 127.6\left(\mathrm{CH}_{\mathrm{SiPh}, \text { meta }}\right), 128.2\left(\mathrm{~d},{ }^{3} J_{\mathrm{CP}}=12.5\right.$ $\left.\mathrm{Hz} ; C \mathrm{H}_{\mathrm{PPh}, \text { meta }}\right), 128.4\left(\mathrm{~d},{ }^{3} J_{\mathrm{CP}}=12.1 \mathrm{~Hz} ; C \mathrm{H}_{\mathrm{PPh}, \text { meta }}\right), 129.9\left(C_{\mathrm{SiPh}, \text { ara }}\right), 130.9\left(\mathrm{~d},{ }^{4} J_{\mathrm{CP}}=2.9 \mathrm{~Hz}\right.$; $\left.C_{\mathrm{PPh}, \text { para }}\right), 131.2\left(\mathrm{~d},{ }^{4} J_{\mathrm{CP}}=2.9 \mathrm{~Hz} ; C_{\mathrm{PPh}, \text { para }}\right), 131.5\left(\mathrm{~d},{ }^{1} J_{\mathrm{CP}}=80.0 \mathrm{~Hz} ; C_{\mathrm{PPh}, \mathrm{ipso}}\right), 131.5\left(\mathrm{~d},{ }^{2} J_{\mathrm{CP}}=9.7\right.$ $\left.\mathrm{Hz} ; C_{\mathrm{PPh}, \text { ortho }}\right), 131.6$ (d, $\left.{ }^{2} J_{\mathrm{CP}}=9.8 \mathrm{~Hz} ; C \mathrm{H}_{\mathrm{PPh}, \text { ortho }}\right), 132.9$ (d, $\left.{ }^{3} J_{\mathrm{CP}}=2.2 \mathrm{~Hz} ; C_{\mathrm{SiPh}, \mathrm{psso}}\right), 135.1\left(\mathrm{~d},{ }^{1} J_{\mathrm{CP}}=\right.$ $\left.84.7 \mathrm{~Hz} ; C_{\text {PPh,ipso }}\right), 137.1$ ( $\left.\mathrm{CH}_{\text {SiPh,ortho }}\right) .{ }^{29} \mathrm{Si}\left\{{ }^{1} \mathrm{H}\right\} \mathrm{NMR}\left(\mathrm{CDCl}_{3}, 99.4 \mathrm{MHz}\right): \delta=-11.8 .{ }^{31} \mathrm{P}\left\{{ }^{1} \mathrm{H}\right\} \mathrm{NMR}$ $\left(\mathrm{CDCl}_{3}, 202.5 \mathrm{MHz}\right): \delta=47.9$. Anal. Calc. for $\mathrm{C}_{31} \mathrm{H}_{26} \mathrm{IPSSi}: \mathrm{C}, 60.39 ; \mathrm{H}, 4.25 ; \mathrm{S}, 5.20$. Found: $\mathrm{C}$, $60.45 ; \mathrm{H}, 4.38 ; \mathrm{S}, 4.92$.

Synthesis of 1d. This complex was synthesized according to the general procedure giving the product as a colorless solid (488 mg, $1.13 \mathrm{mmol}, 68 \%) .{ }^{1} \mathrm{H} \mathrm{NMR}\left(\mathrm{CDCl}_{3}, 500.1 \mathrm{MHz}\right): \delta=0.09$ (br, 9H; $\left.\mathrm{CH}_{3}\right)$, $3.95\left(\mathrm{~d},{ }^{2} J_{\mathrm{HP}}=10.2 \mathrm{~Hz}, 1 \mathrm{H} ; \mathrm{CHI}\right), 7.42-7.51\left(\mathrm{~m}, 6 \mathrm{H} ; \mathrm{CH}_{\mathrm{PPh}, \text { meta }}\right.$ and $\left.\mathrm{PPh}_{\mathrm{para}}\right), 7.97-8.01(\mathrm{~m}, 2 \mathrm{H}$; $\left.\mathrm{C}_{\mathrm{PPh}, \text { ortho }}\right), 8.03-8.08$ (m, 2H; $\left.\mathrm{CH}_{\mathrm{PPh}, \text { ortho }}\right) .{ }^{13} \mathrm{C}\left\{{ }^{1} \mathrm{H}\right\} \mathrm{NMR}\left(\mathrm{CDCl}_{3}, 125.8 \mathrm{MHz}\right): \delta=0.8\left(\mathrm{~d},{ }^{3} J_{\mathrm{CP}}=1.7\right.$ $\left.\mathrm{Hz} ; \mathrm{CH}_{3}\right), 11.2\left(\mathrm{~d},{ }^{1} J_{\mathrm{CP}}=33.1 \mathrm{~Hz} ; \mathrm{CHI}\right), 128.4\left(\mathrm{~d},{ }^{3} J_{\mathrm{CP}}=12.5 \mathrm{~Hz} ; C_{\mathrm{PPh}, \text { meta }}\right), 128.7$ (d, ${ }^{3} J_{\mathrm{CP}}=12.0 \mathrm{~Hz}$; $C_{\mathrm{PPh}, \text { meta }}$ ), 131.6 (d, $\left.{ }^{4} J_{\mathrm{CP}}=3.0 \mathrm{~Hz} ; C_{\mathrm{PPh}, \text { para }}\right), 131.6$ (d, $\left.{ }^{4} J_{\mathrm{CP}}=3.0 \mathrm{~Hz} ; C \mathrm{H}_{\mathrm{PPh}, \text { para }}\right), 131.7$ (d, ${ }^{2} J_{\mathrm{CP}}=10.0$ $\left.\mathrm{Hz} ; C_{\mathrm{PPh}, \text { ortho }}\right), 131.7$ (d, $\left.{ }^{2} J_{\mathrm{CP}}=9.8 \mathrm{~Hz} ; C \mathrm{H}_{\mathrm{PPh}, \text { ortho }}\right), 132.9$ (d, $\left.{ }^{1} J_{\mathrm{CP}}=79.9 \mathrm{~Hz} ; C_{\mathrm{PPh}, \mathrm{psso}}\right), 133.6\left(\mathrm{~d},{ }^{1} J_{\mathrm{CP}}=\right.$ 
83.2 Hz; $\left.C_{\text {PPh,ipso }}\right) .{ }^{29} \mathrm{Si}\left\{{ }^{1} \mathrm{H}\right\}$ NMR $\left(\mathrm{CDCl}_{3}, 99.4 \mathrm{MHz}\right): \delta=9.1 .{ }^{31} \mathrm{P}\left\{{ }^{1} \mathrm{H}\right\} \mathrm{NMR}\left(\mathrm{CDCl}_{3}, 202.5 \mathrm{MHz}\right): \delta$ = 46.6. Anal. Calc. for $\mathrm{C}_{16} \mathrm{H}_{20} \mathrm{IPSSi}$ : C, 44.65; H, 4.68; S, 7.45. Found: C, 45.02; H, 4.68; S, 7.49.

Synthesis of 1e. This complex was synthesized according to the general procedure giving the product as a colorless solid $(1.10 \mathrm{~g}, 2.48 \mathrm{mmol}, 57 \%) .{ }^{1} \mathrm{H} \mathrm{NMR}\left(\mathrm{CDCl}_{3}, 400.1 \mathrm{MHz}\right): \delta=0.16\left(\mathrm{~s}, 3 \mathrm{H} ; \mathrm{SiCH}_{3}\right)$, 0.44 (s, 3H; $\left.\mathrm{SiCH}_{3}\right), 2.71\left(\mathrm{~s}, 6 \mathrm{H} ; \mathrm{N}\left(\mathrm{CH}_{3}\right)_{2}\right), 5.11\left(\mathrm{br} \mathrm{d},{ }^{2} J_{\mathrm{HP}}=10.0 \mathrm{~Hz}, 1 \mathrm{H} ; \mathrm{CHCl}\right), 7.19\left(\mathrm{br} \mathrm{d},{ }^{3} J_{\mathrm{HH}}=\right.$ $\left.7.2 \mathrm{~Hz}, 1 \mathrm{H} ; \mathrm{CH}_{\text {Aniline }}\right), 7.34$ (br d, $\left.1 \mathrm{H} ; \mathrm{CH}_{\text {Aniline }}\right), 7.41-7.55$ (m, 8H; $\left.\mathrm{CH}_{\text {arom. }}\right), 7.88-7.93$ (m, $2 \mathrm{H}$, $\left.\mathrm{C}_{\mathrm{PPh}, \text { ortho }}\right), 8.07-8.12$ (m, 2H; $\left.\mathrm{CH}_{\mathrm{PPh}, \text { ortho }}\right) .{ }^{13} \mathrm{C}\left\{{ }^{1} \mathrm{H}\right\}-\mathrm{NMR}\left(\mathrm{CDCl}_{3}, 100.6 \mathrm{MHz}\right): \delta=-3.1\left(\mathrm{SiCH}_{3}\right), 0.1$ $\left(\mathrm{SiCH}_{3}\right), 42.1\left(\mathrm{~d},{ }^{1} J_{\mathrm{CP}}=37.8 \mathrm{~Hz} ; \mathrm{CHCl}\right), 47.3\left(\mathrm{~N}\left(\mathrm{CH}_{3}\right)_{2}\right), 122.2\left(\mathrm{CH}_{\text {Aniline }}\right), 125.7\left(\mathrm{CH}_{\text {Aniline }}\right), 128.3(\mathrm{~d}$, $\left.{ }^{3} J_{\mathrm{CP}}=12.3 \mathrm{~Hz} ; C \mathrm{H}_{\mathrm{PPh}, \mathrm{meta}}\right), 128.6\left(\mathrm{~d},{ }^{3} J_{\mathrm{CP}}=12.2 \mathrm{~Hz} ; C \mathrm{H}_{\mathrm{PPh}, \mathrm{meta}}\right), 131.2\left(\mathrm{~d},{ }^{1} J_{\mathrm{CP}}=81.8 \mathrm{~Hz} ; C_{\mathrm{PPh}, \mathrm{pss}}\right)$, $131.3\left(\mathrm{CH}_{\text {Aniline }}\right), 131.5\left(\mathrm{~d},{ }^{2} J_{\mathrm{CP}}=9.7 \mathrm{~Hz} ; C \mathrm{H}_{\mathrm{PPh}, \text { ortho }}\right), 131.6\left(\mathrm{~d},{ }^{4} J_{\mathrm{CP}}=3.0 \mathrm{~Hz} ; C \mathrm{H}_{\mathrm{PPh}, \text { para }}\right), 131.8\left(\mathrm{~d},{ }^{4} J_{\mathrm{CP}}\right.$ $\left.=3.0 \mathrm{~Hz} ; C_{\mathrm{PPh}, \text { para }}\right), 132.7$ (d, $\left.{ }^{2} J_{\mathrm{CP}}=9.9 \mathrm{~Hz} ; C_{\mathrm{PPh}, \text { ortho }}\right), 133.7\left(\mathrm{~d},{ }^{1} J_{\mathrm{CP}}=81.9 \mathrm{~Hz} ; C_{\mathrm{PPh}, \mathrm{pss}}\right), 134.5$ $\left(C_{\text {Aniline }}\right), 136.0\left(\mathrm{CH}_{\text {Aniline }}\right), 160.8\left(\mathrm{COCH}_{3 \text { Aniline }}\right) .{ }^{29} \mathrm{Si}\left\{{ }^{1} \mathrm{H}\right\} \mathrm{NMR}\left(\mathrm{CDCl}_{3}, 59.6 \mathrm{MHz}\right): \delta=0.3\left(\mathrm{~d},{ }^{2} J_{\mathrm{SiP}}=\right.$ $3.2 \mathrm{~Hz}) .{ }^{31} \mathrm{P}\left\{{ }^{1} \mathrm{H}\right\} \mathrm{NMR}\left(\mathrm{CDCl}_{3}, 162.0 \mathrm{MHz}\right): \delta=45.9$. Anal. Calc. for $\mathrm{C}_{23} \mathrm{H}_{27} \mathrm{ClNPSSi}: \mathrm{C}, 62.21 ; \mathrm{H}$, $6.13 ; \mathrm{N}, 3.15 ; \mathrm{S}, 7.22$. Found: C, 62.05; H, 5.97; N, 3.04; S, 7.29.

Synthesis of 1f. This complex was synthesized according to the general procedure giving the product as a colorless solid $(273 \mathrm{mg}, 492 \mu \mathrm{mol}, 84 \%)$. ${ }^{1} \mathrm{H} \mathrm{NMR}\left(\mathrm{CDCl}_{3}, 400.1 \mathrm{MHz}\right): \delta=3.63\left(\mathrm{~s}, 3 \mathrm{H}: \mathrm{OCH}_{3}\right)$. $5.51\left(\mathrm{~d},{ }^{2} J_{\mathrm{HP}}=11.3 \mathrm{~Hz}, 1 \mathrm{H} ; \mathrm{CHCl}\right), 6.73\left(\mathrm{br} \mathrm{d},{ }^{3} J_{\mathrm{HH}}=8.2 \mathrm{~Hz}, 1 \mathrm{H} ; \mathrm{CH}_{\text {Anisole }}\right), 6.90\left(\mathrm{td},{ }^{3} J_{\mathrm{HH}}=7.3 \mathrm{~Hz}\right.$, $\left.{ }^{5} J_{\mathrm{HH}}=0.8 \mathrm{~Hz}, 1 \mathrm{H} ; \mathrm{CH}_{\text {Anisole }}\right), 7.17-7.22\left(\mathrm{~m}, 2 \mathrm{H} ; \mathrm{CH}_{\mathrm{SiPh}, \text { para }}\right), 7.23-7.38\left(\mathrm{~m}, 11 \mathrm{H} ; \mathrm{CH}_{\text {arom. }}\right), 7.67-7.77$ (m, $8 \mathrm{H}$; $\mathrm{CH}_{\mathrm{PPh}, \text { ortho }}$ and $\left.\mathrm{CH}_{\mathrm{SiPh}, \text { ortho }}\right){ }^{13} \mathrm{C}\left\{{ }^{1} \mathrm{H}\right\} \mathrm{NMR}\left(\mathrm{CDCl}_{3}, 125.8 \mathrm{MHz}\right): \delta=41.7\left(\mathrm{~d},{ }^{1} J_{\mathrm{CP}}=39.3 \mathrm{~Hz}\right.$; $C \mathrm{HCl}), 55.1\left(\mathrm{OCH}_{3}\right) 110.3\left(\mathrm{CH}_{\text {Anisole }}\right), 120.8\left(\mathrm{CH}_{\text {Anisole }}\right), 122.4\left(\mathrm{~d},{ }^{3} J_{\mathrm{CP}}=2.6 \mathrm{~Hz} ; C_{\text {Anisole }}\right), 127.1$ ( $\left.C \mathrm{H}_{\mathrm{SiPh}, \mathrm{meta}}\right), 127.4\left(\mathrm{CH}_{\mathrm{SiPh}, \mathrm{meta}}\right), 127.8\left(\mathrm{~d},{ }^{3} J_{\mathrm{CP}}=12.5 \mathrm{~Hz} ; C \mathrm{H}_{\mathrm{PPh}, \text { meta }}\right), 128.3\left(\mathrm{~d},{ }^{3} J_{\mathrm{CP}}=12.2 \mathrm{~Hz}\right.$; $\left.C \mathrm{H}_{\mathrm{PPh}, \text { meta }}\right), 129.6\left(\mathrm{CH}_{\mathrm{SiPh}, \text { para }}\right), 129.7\left(\mathrm{CH}_{\mathrm{SiPh}, \mathrm{para}}\right), 130.6$ (d, $\left.{ }^{1} J_{\mathrm{CP}}=83.6 \mathrm{~Hz} ; C_{\mathrm{PPh}, \mathrm{psso}}\right), 131.1\left(\mathrm{~d},{ }^{4} J_{\mathrm{CP}}=2.5\right.$

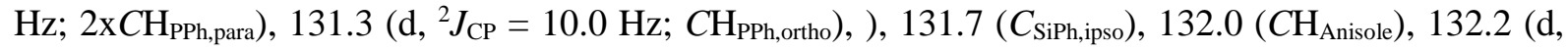
$\left.{ }^{3} J_{\mathrm{CP}}=2.7 \mathrm{~Hz} ; C_{\mathrm{SiPh}, \mathrm{pso}}\right), 133.0\left(\mathrm{~d},{ }^{2} J_{\mathrm{CP}}=10.0 \mathrm{~Hz} ; C \mathrm{H}_{\mathrm{PPh}, \text { ortho }}\right), 134.0\left(\mathrm{~d},{ }^{1} J_{\mathrm{CP}}=82.3 \mathrm{~Hz} ; C_{\mathrm{PPh}, \mathrm{psso}}\right), 136.9$ $\left(\mathrm{CH}_{\text {SiPh,ortho }}\right), 137.5\left(\mathrm{CH}_{\text {SiPh,ortho }}\right), 137.7\left(\mathrm{CH}_{\text {Anisole }}\right), 164.1\left(\mathrm{COCH}_{3 \text { Anisole }}\right) .{ }^{29} \mathrm{Si}\left\{{ }^{1} \mathrm{H}\right\} \mathrm{NMR}\left(\mathrm{CDCl}_{3}, 79.5\right.$ MHz): $\delta=-12.7\left(\mathrm{~d},{ }^{2} J_{\mathrm{SiP}}=2.5 \mathrm{~Hz}\right) .{ }^{31} \mathrm{P}\left\{{ }^{1} \mathrm{H}\right\} \mathrm{NMR}\left(\mathrm{CDCl}_{3}, 162.0 \mathrm{MHz}\right): \delta=46.3$. Anal. Calc. for $\mathrm{C}_{32} \mathrm{H}_{28} \mathrm{ClOPSSi}$ C, 69.23; H, 5.08; S, 5.78. Found: C, 68.94; H, 5.05; S, 5.61. 


\section{NMR spectra of the isolated compounds}

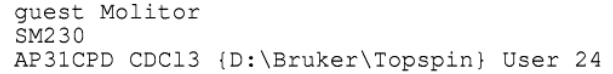

Figure S1. ${ }^{31} \mathrm{P}\left\{{ }^{1} \mathrm{H}\right\}$ NMR and ${ }^{1} \mathrm{H}$ NMR spectra of silane $\mathbf{1 b}$. 


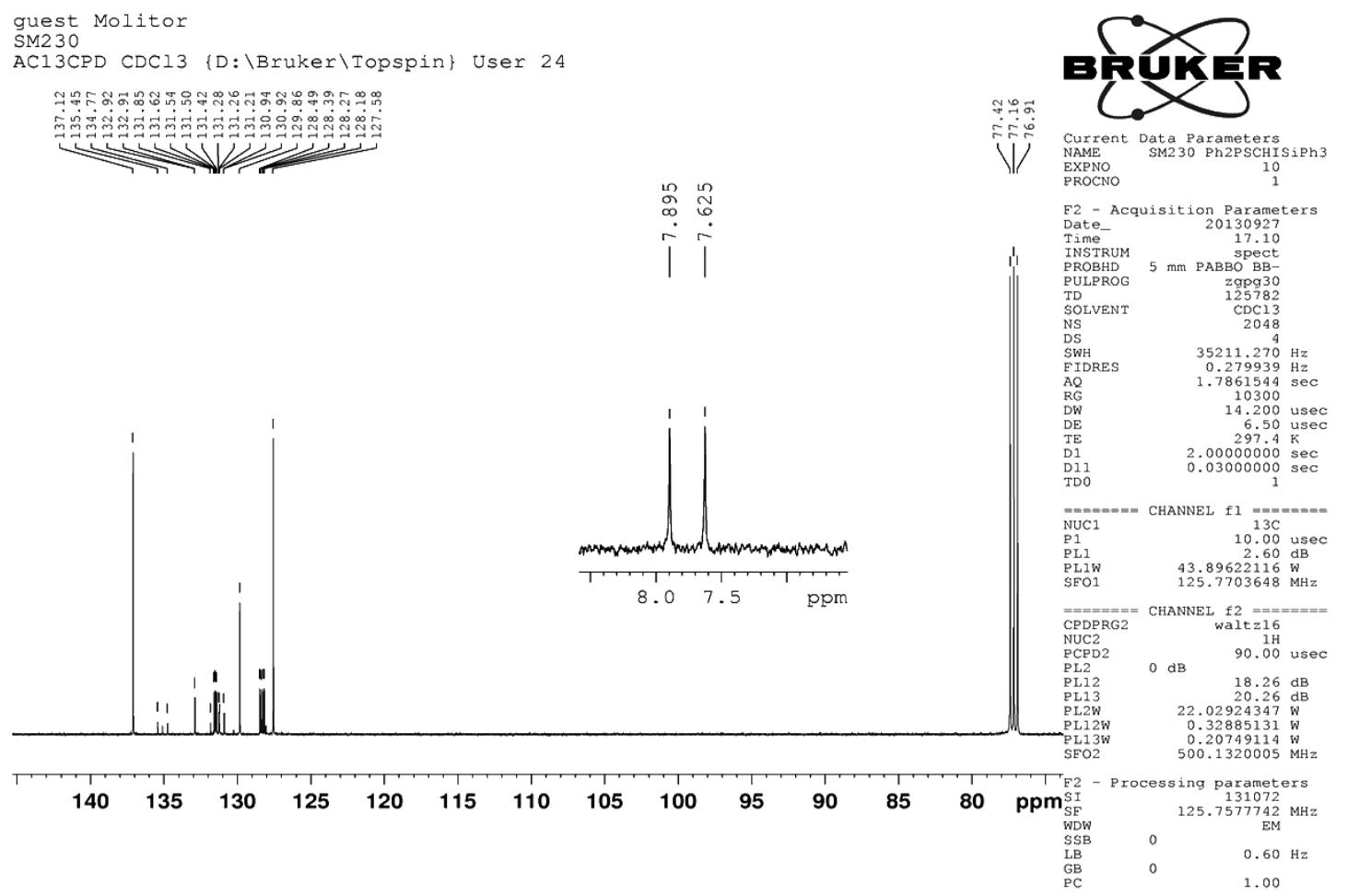

guest Molitor

SM2 30

AC13DEPT135 CDC13 \{D: \Bruker\Topspin\} User 24

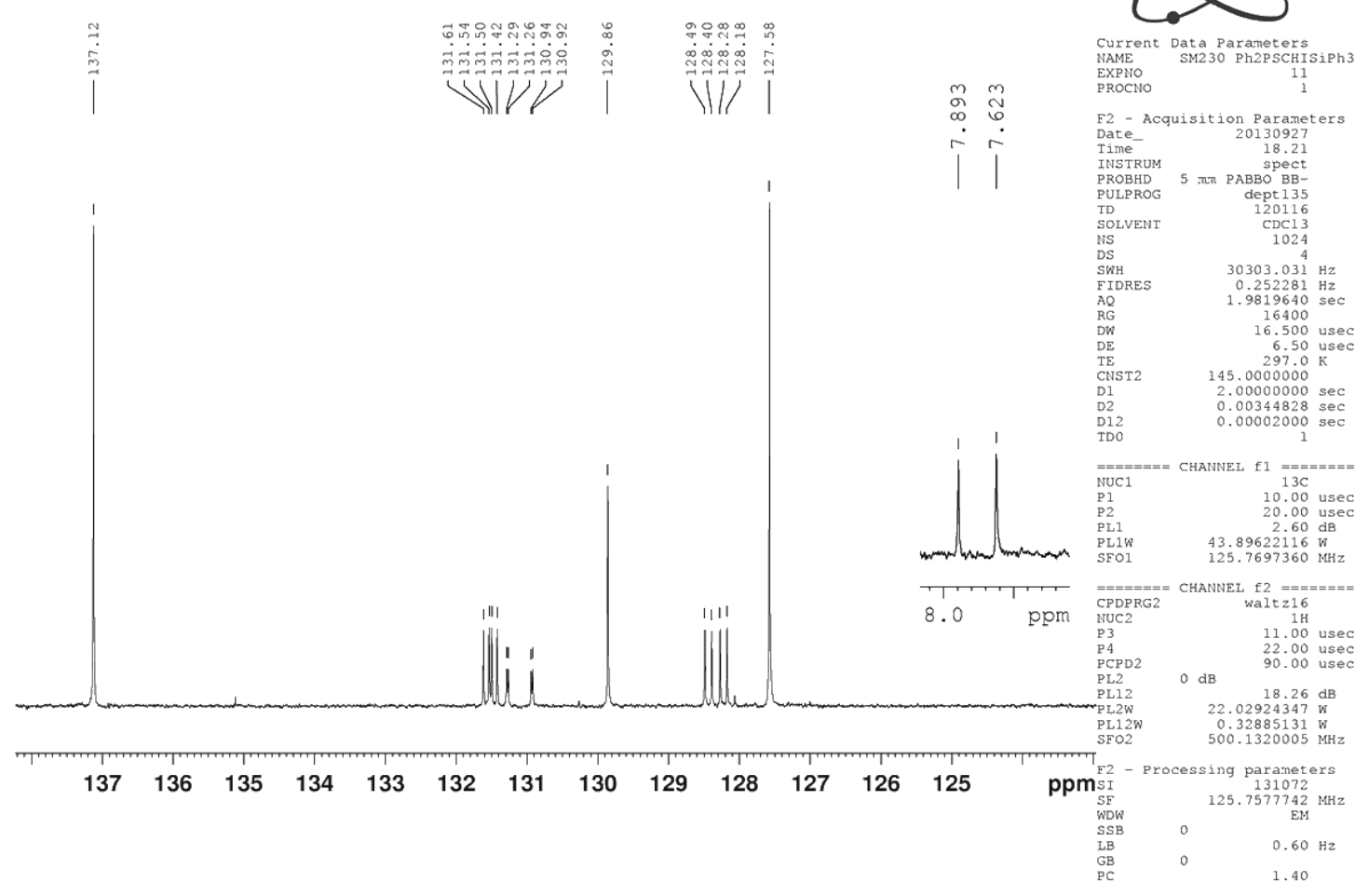

Figure S2. ${ }^{13} \mathrm{C}\left\{{ }^{1} \mathrm{H}\right\}$ NMR and ${ }^{13} \mathrm{C}\left\{{ }^{1} \mathrm{H}\right\}$-DEPT135 NMR spectra of silane $\mathbf{1 b}$. 


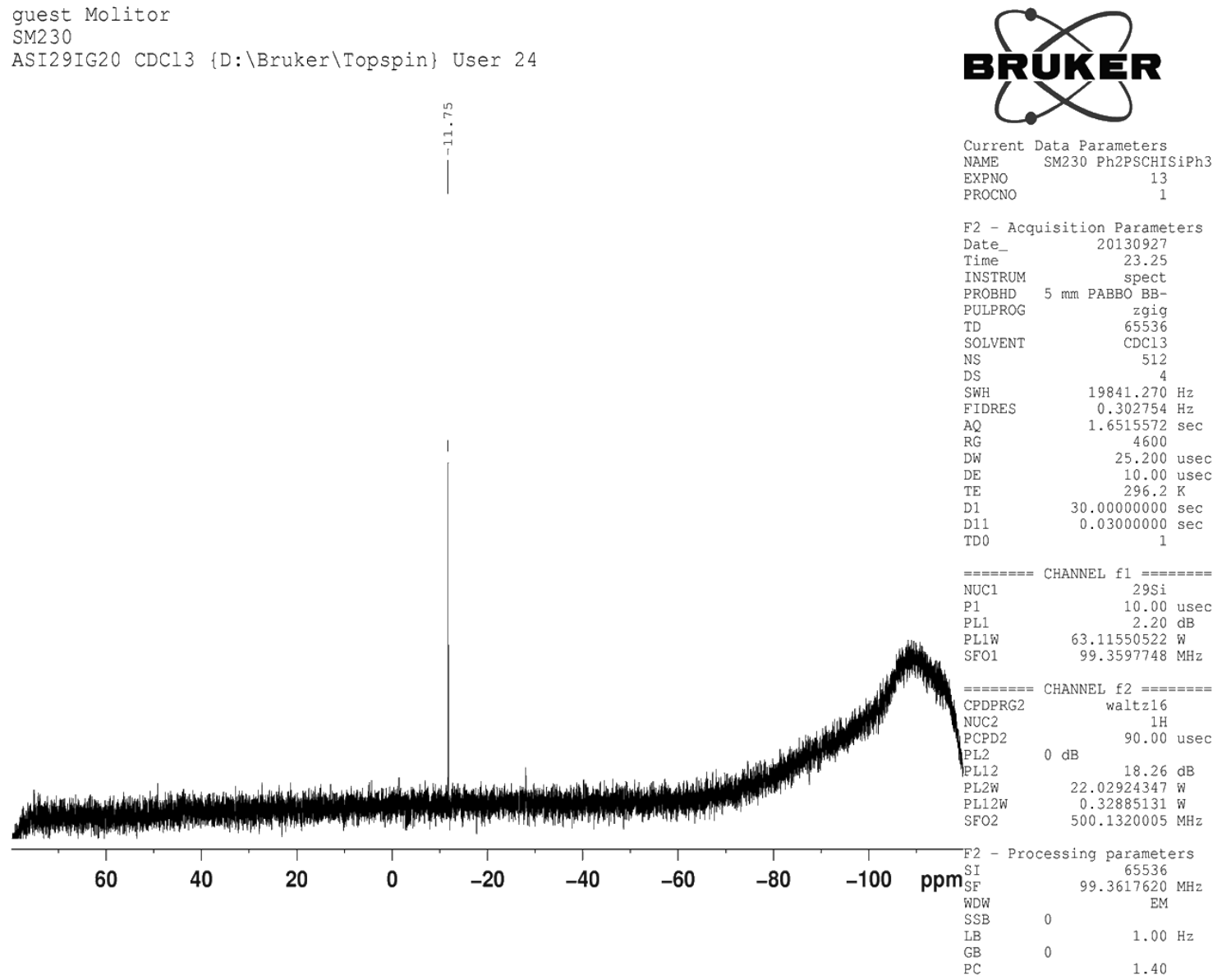

Figure S3. ${ }^{29} \mathrm{Si}\left\{{ }^{1} \mathrm{H}\right\}$ NMR spectrum of silane $\mathbf{1 b}$. 

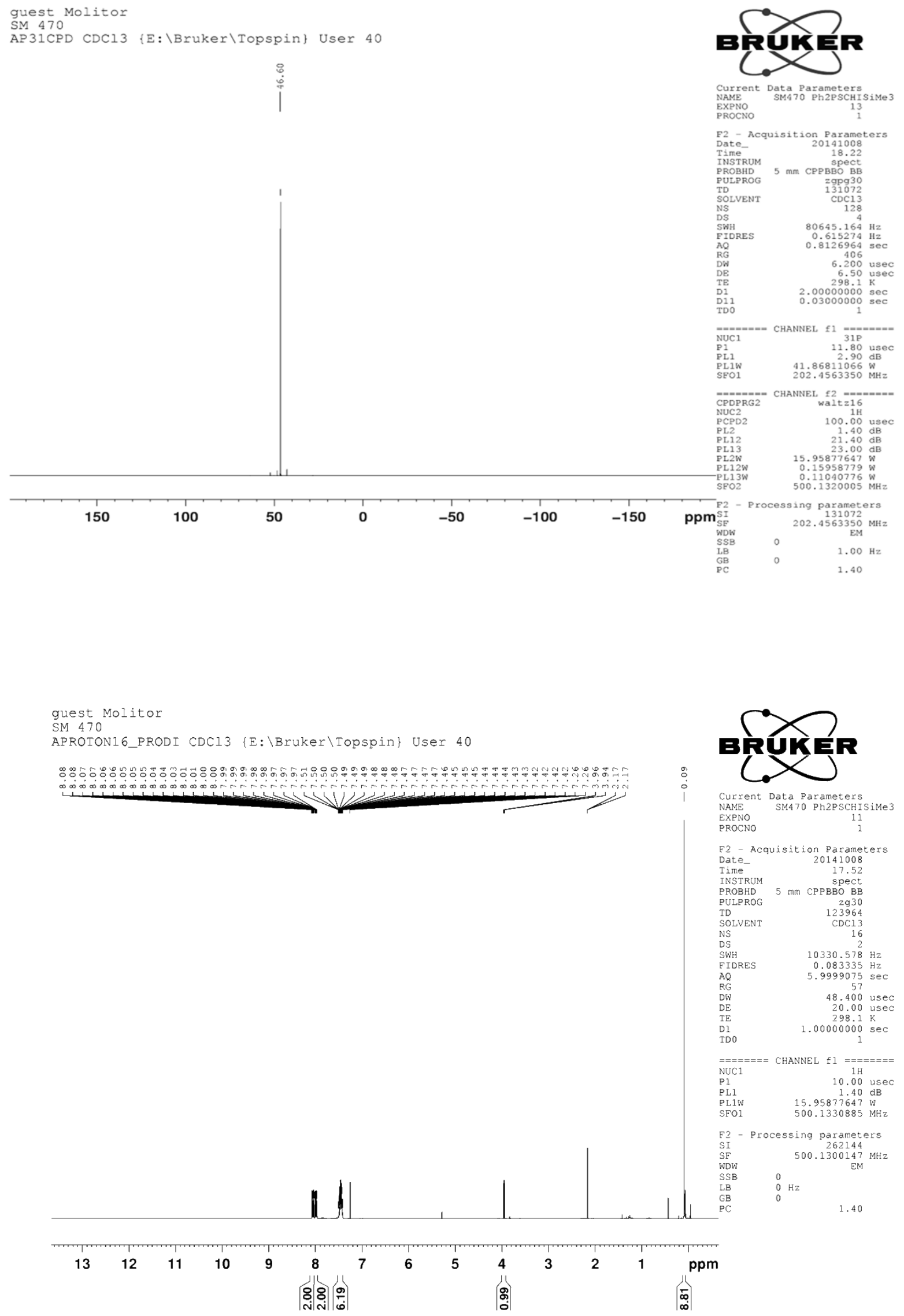

Figure S4. ${ }^{31} \mathrm{P}\left\{{ }^{1} \mathrm{H}\right\}$ NMR and ${ }^{1} \mathrm{H}$ NMR spectra of silane $\mathbf{1 d}$. 


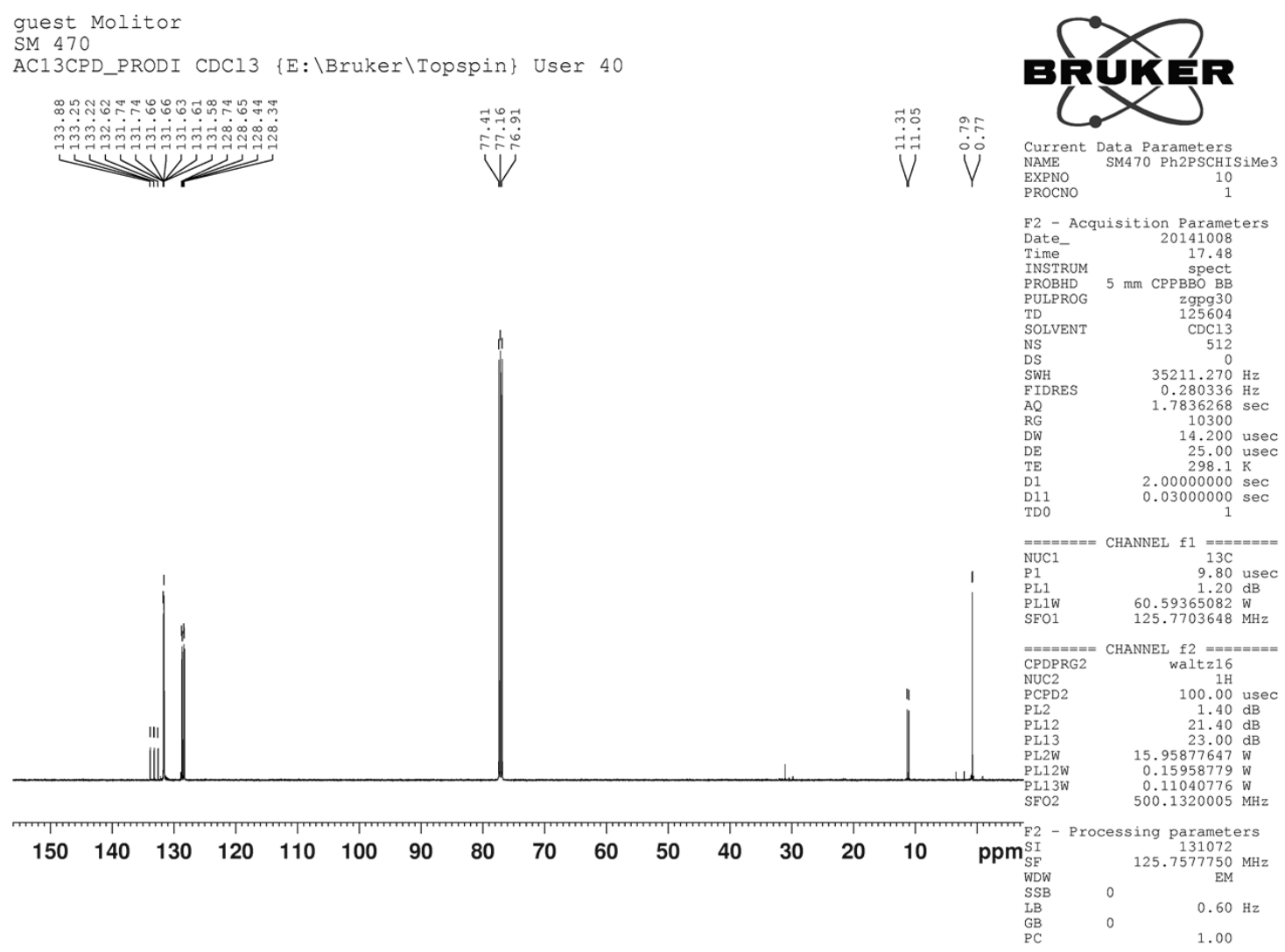

guest Molitor

SM 470

AC13DEPT135_PRODI CDC13 \{E:\Bruker $\backslash$ Topspin\} User 40

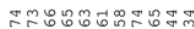
लिलंलें 幽
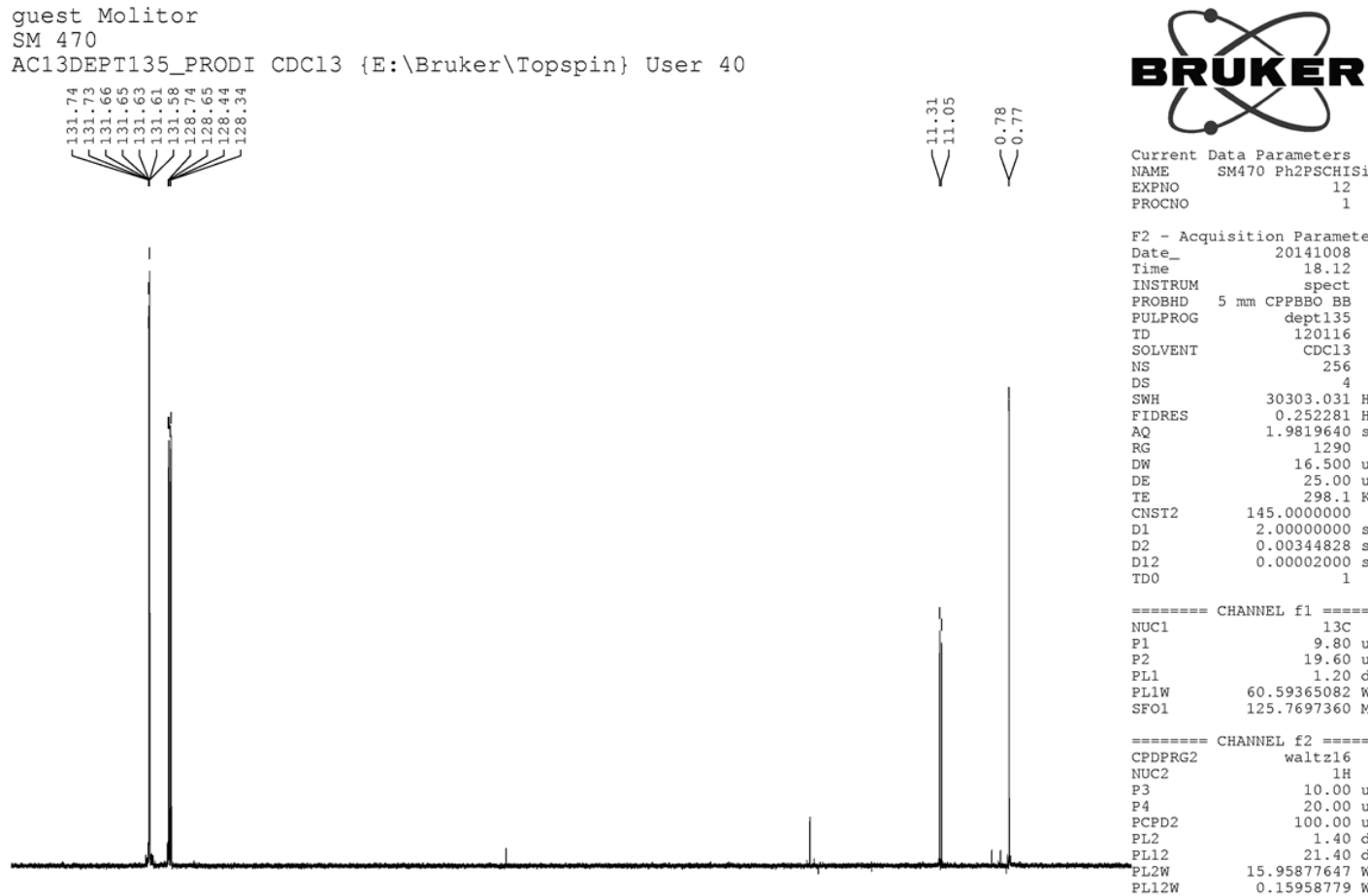

Current Data Parameters
NAME SM470 Ph2PSCHSiMe3 NAME
EXPNO
PROCNO

F2 - Acquisition Parameters

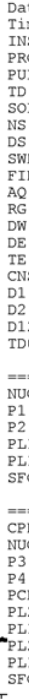

INSTRUM

PULPROG
TD

SOLV
NS
DS

$\mathrm{CDCl}_{2} 3$
256

$\begin{array}{ll}\text { SWH } & 30303.031 \mathrm{~Hz} \\ \text { FIDRES } & 0.252281 \mathrm{~Hz}\end{array}$

$\begin{array}{lr}1.9819640 \mathrm{sec} \\ \text { AQ } & 1290 \mathrm{use}\end{array}$

$\begin{array}{ll}25.00 \mathrm{u} \\ \mathrm{DE} & 298.1 \mathrm{~K}\end{array}$

145.0000000
$2.00000000 \mathrm{sec}$

$0.00344828 \mathrm{sec}$
$0.00002000 \mathrm{sec}$

$== \pm==$ CHANNEL $\mathrm{f} 1 \mathrm{1}== \pm= \pm=$

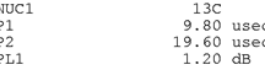

$\begin{array}{ll}60.59365082 \mathrm{~W} \\ \text { PL1W } & 125.7697360 \mathrm{MHz}\end{array}$

$==== \pm$ CHANNEL f $2====$
waltz16

3

$\begin{array}{llllllllllllllll}140 & 130 & 120 & 110 & 100 & 90 & 80 & 70 & 60 & 50 & 40 & 30 & 20 & 10 & 0 & \mathrm{ppm}\end{array}$

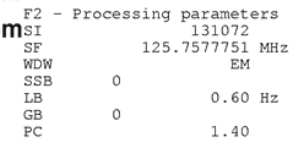

Figure S5. ${ }^{13} \mathrm{C}\left\{{ }^{1} \mathrm{H}\right\}$ NMR and ${ }^{13} \mathrm{C}\left\{{ }^{1} \mathrm{H}\right\}$-DEPT135 NMR spectra of silane $\mathbf{1 d}$. 


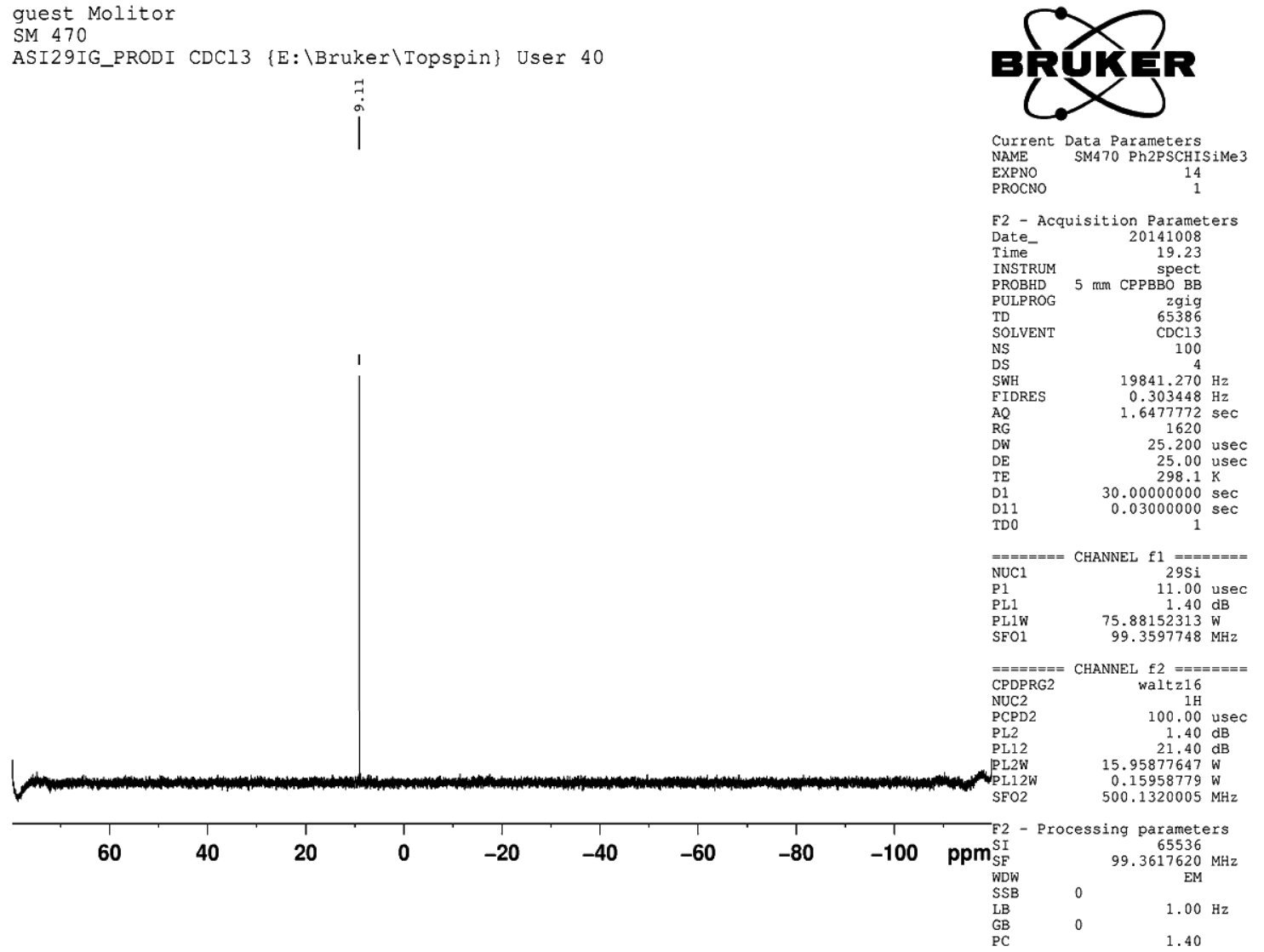

Figure S6. ${ }^{29} \mathrm{Si}\left\{{ }^{1} \mathrm{H}\right\}$ NMR spectrum of silane $\mathbf{1 d}$. 
SM080C sauberes Produkt

AP31CPD CDC13 \{D: \Topspin21\} User 12
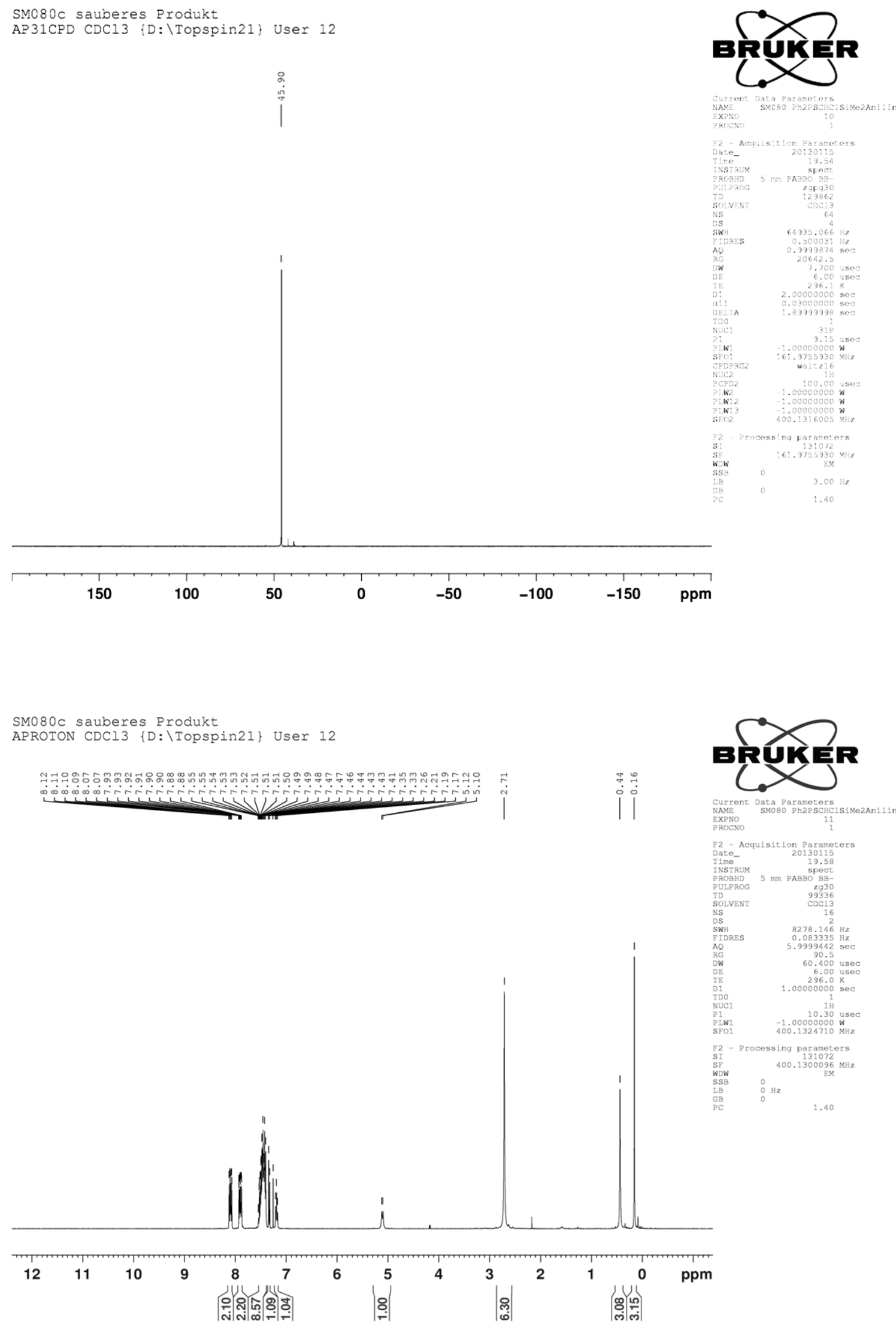

Figure S7. ${ }^{31} \mathrm{P}\left\{{ }^{1} \mathrm{H}\right\}$ NMR and ${ }^{1} \mathrm{H}$ NMR spectra of silane $1 \mathrm{e}$. 


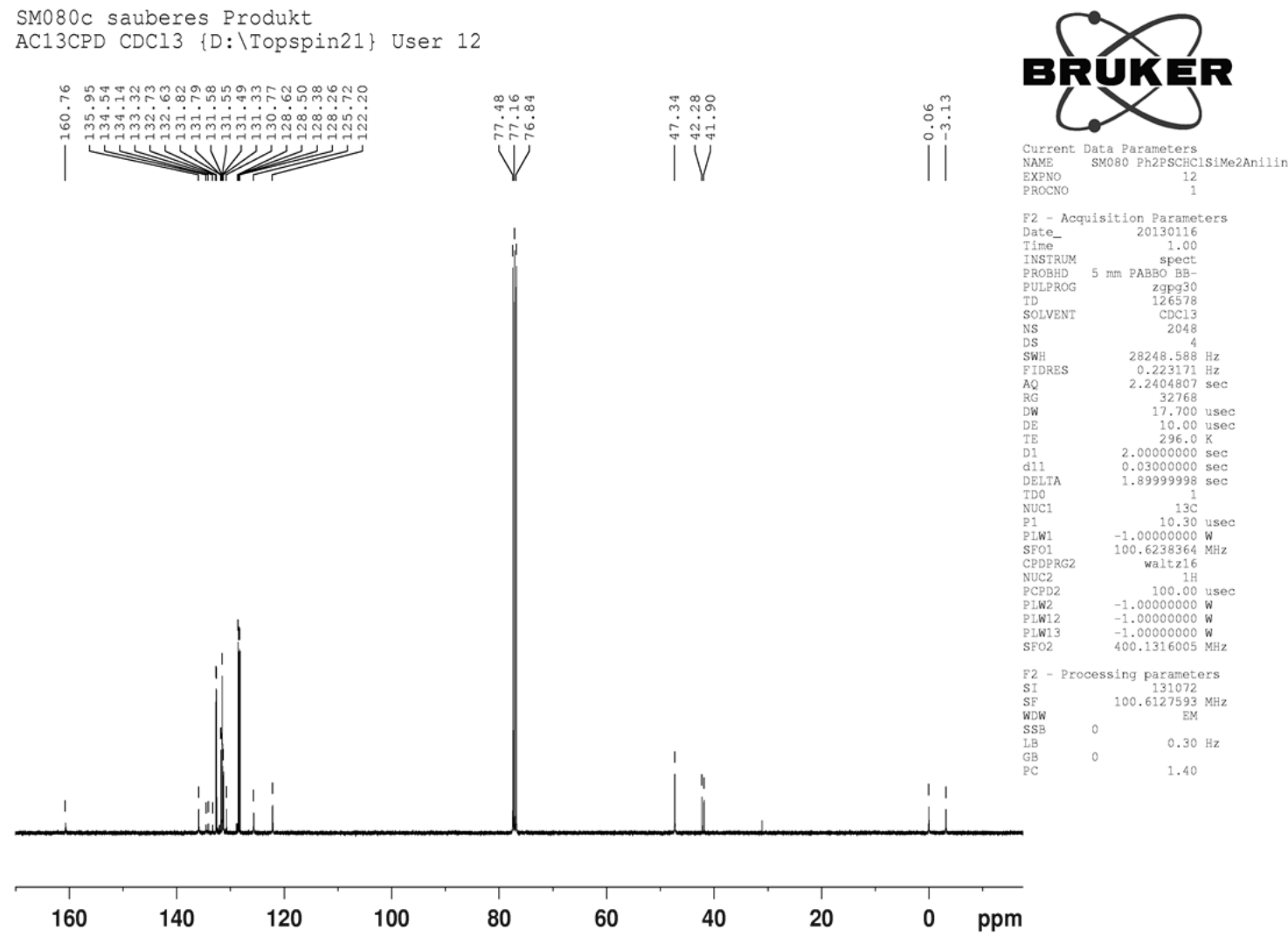

SM080C sauberes Produkt AC13DEPT135 CDC13 $\{$ : $\backslash$ Topspin21\} User 12

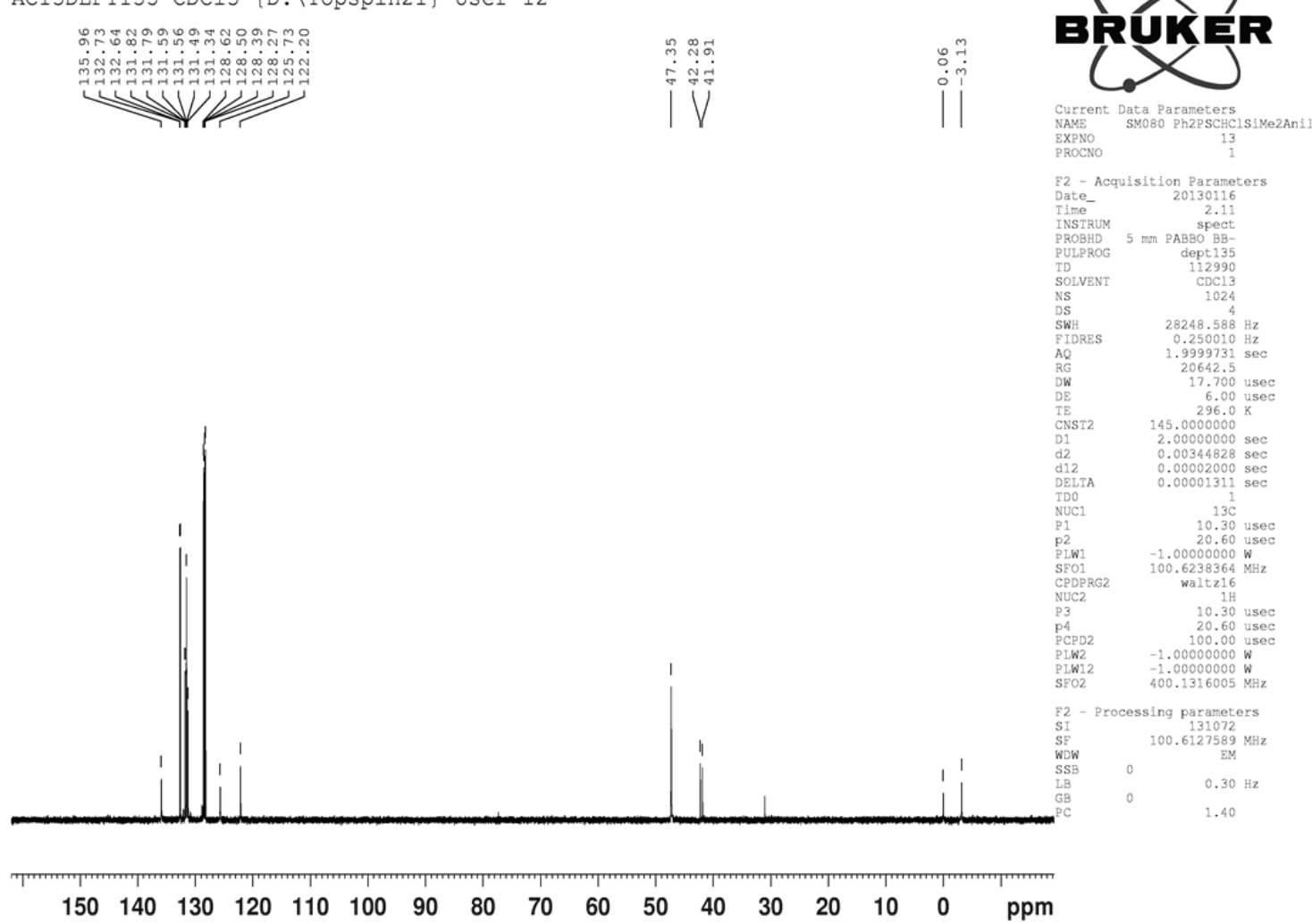

Figure S8. ${ }^{13} \mathrm{C}\left\{{ }^{1} \mathrm{H}\right\}$ NMR and ${ }^{13} \mathrm{C}\left\{{ }^{1} \mathrm{H}\right\}$-DEPT135 NMR spectra of silane 1e. 


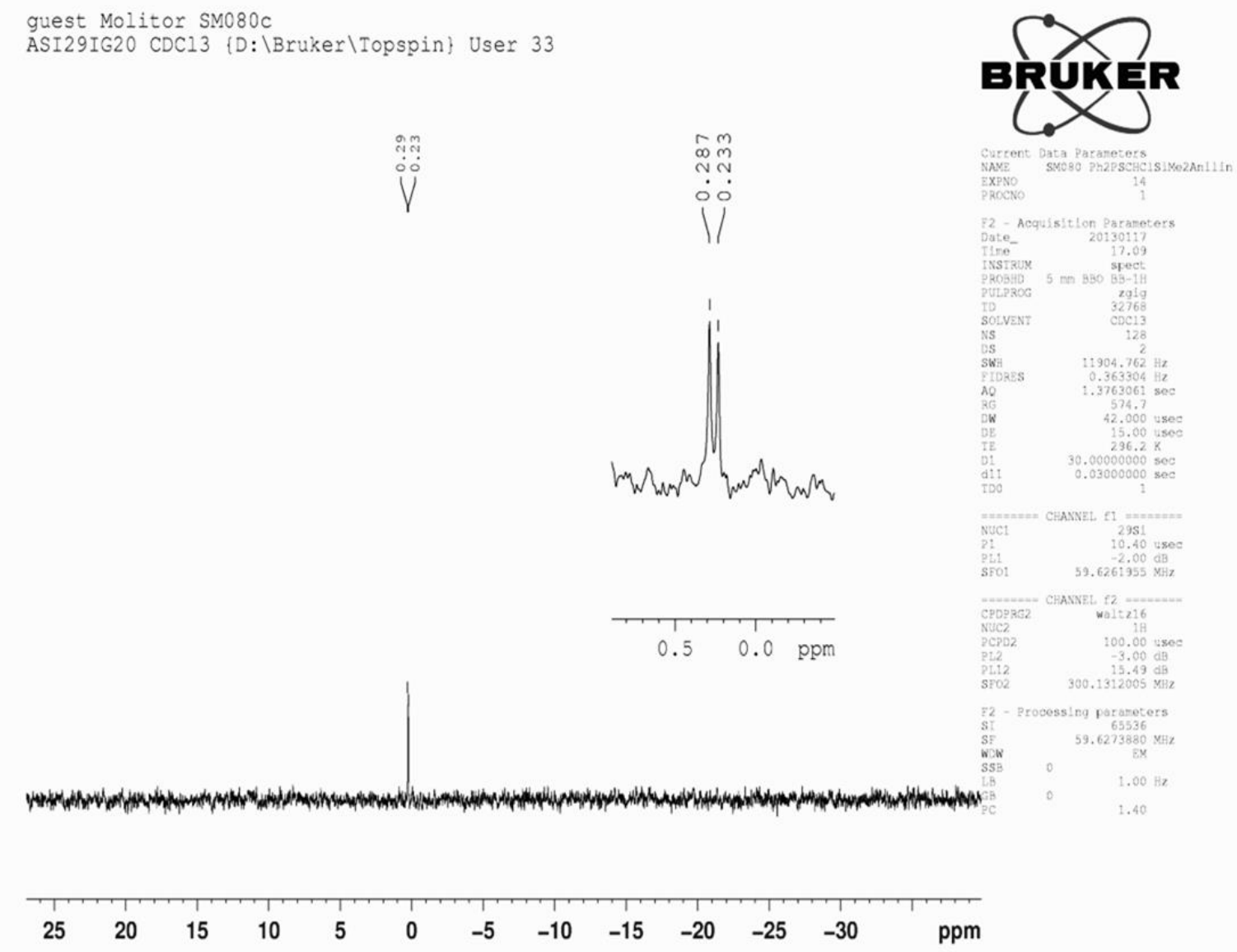

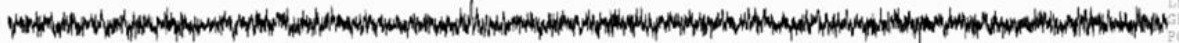

$$
\text { ำ }
$$

Figure S9. ${ }^{29} \mathrm{Si}\left\{{ }^{1} \mathrm{H}\right\}$ NMR spectrum of silane $\mathbf{1 e}$. 
SM075C Produkt zur Charakterisierung AP 31 CPD CDC13 \{D:\Topspin21\} User 30
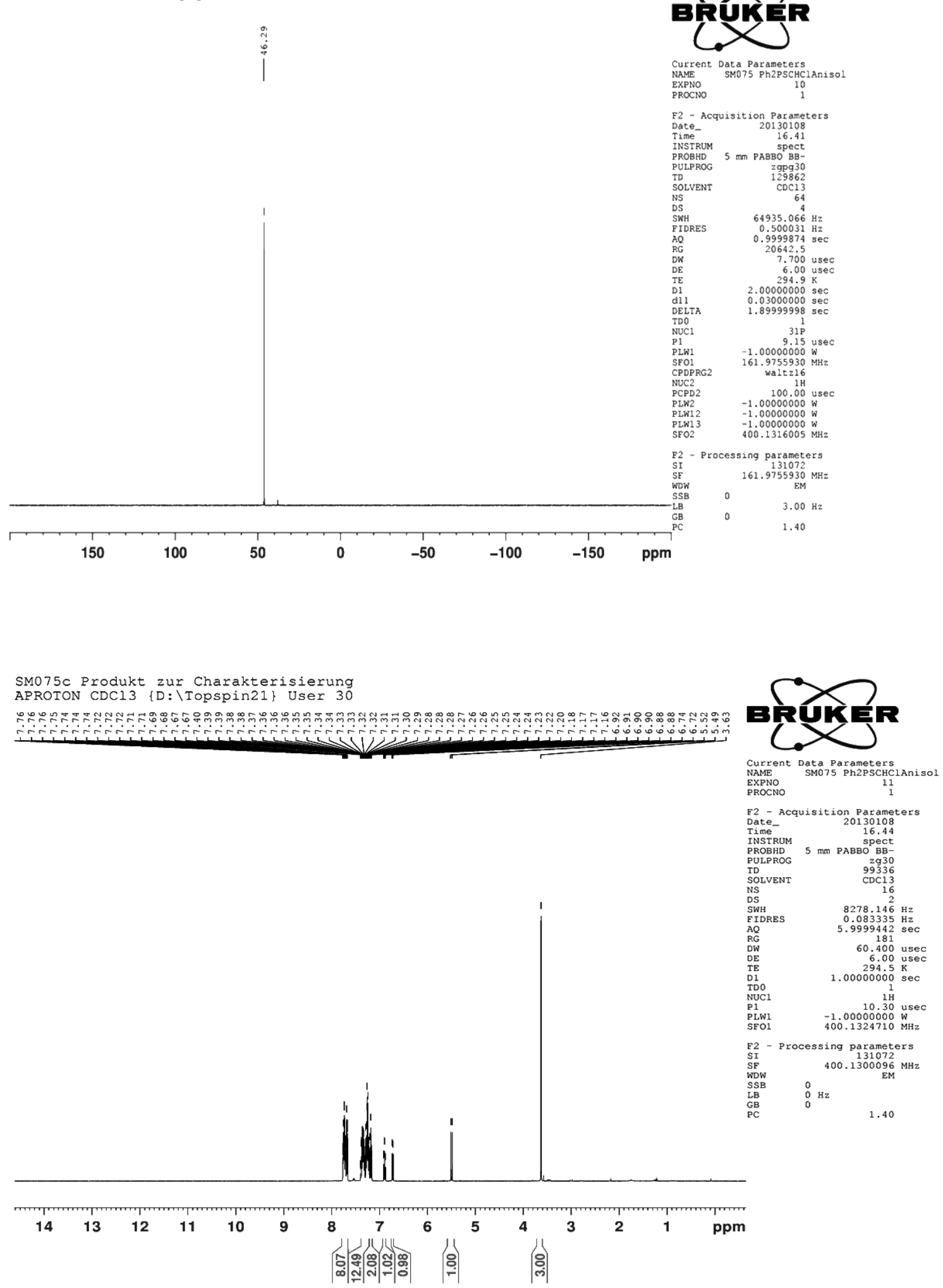

Figure S10. ${ }^{31} \mathrm{P}\left\{{ }^{1} \mathrm{H}\right\}$ NMR and ${ }^{1} \mathrm{H}$ NMR spectra of silane 1f. 


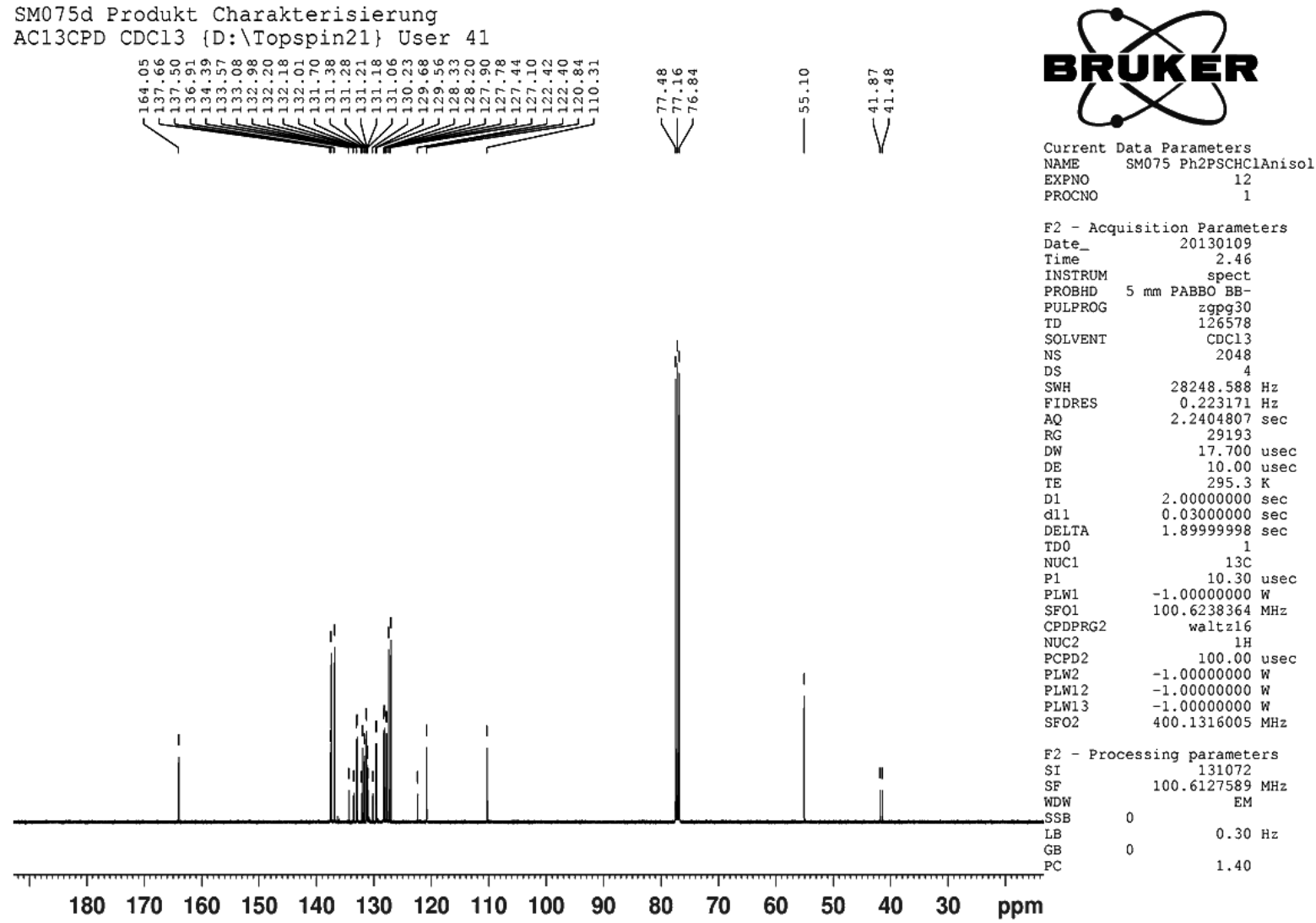

SM075d Produkt Charakterisierung AC13DEPT135 CDC13 $\{$ D: \Topspin21\} User 41

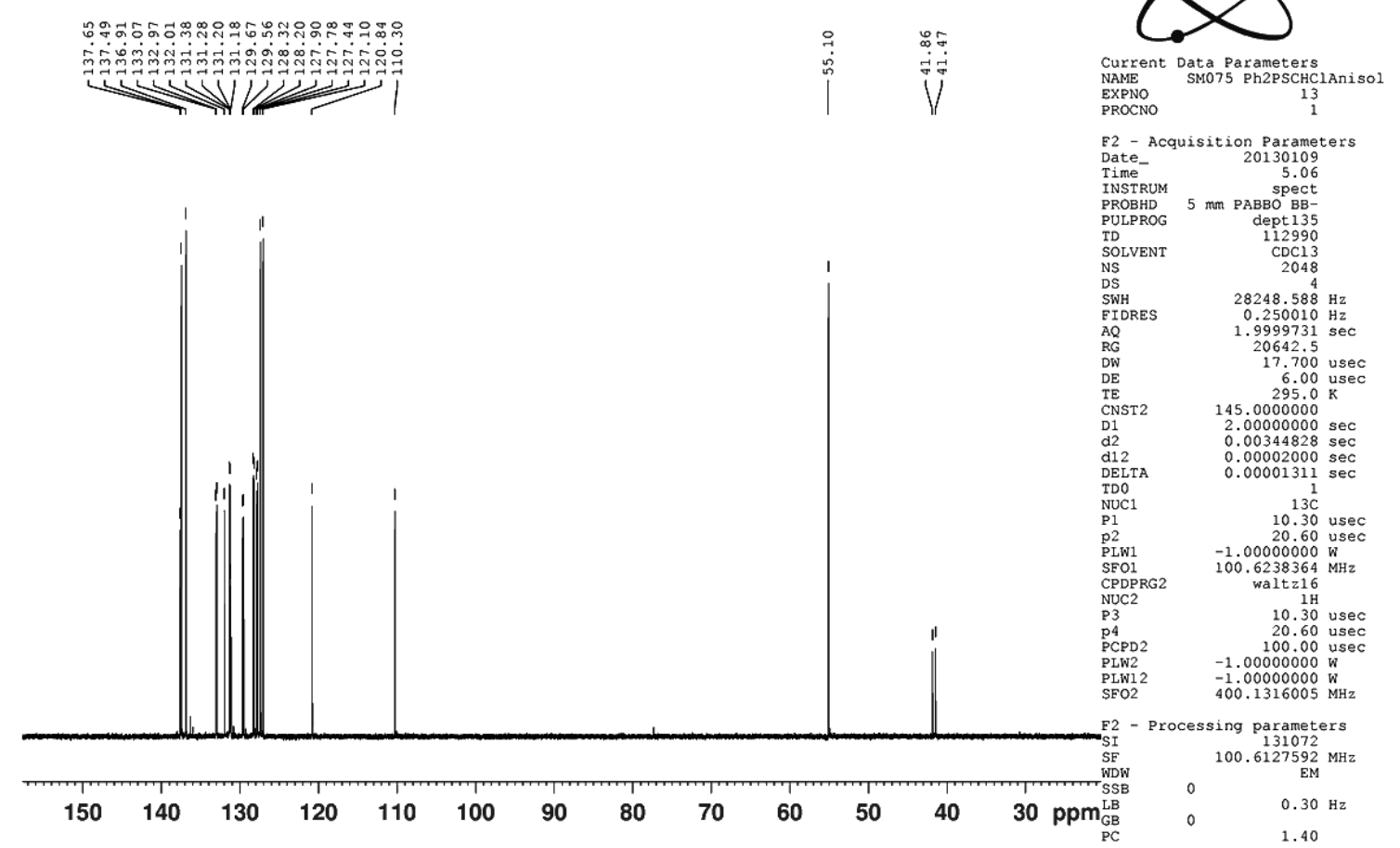

Figure S11. ${ }^{13} \mathrm{C}\left\{{ }^{1} \mathrm{H}\right\}$ NMR and ${ }^{13} \mathrm{C}\left\{{ }^{1} \mathrm{H}\right\}$-DEPT135 NMR spectra of silane $\mathbf{1 f}$. 
SM075c saubers Produkt

ASI29IG20 CDC13 \{D: \Topspin21\} User 11

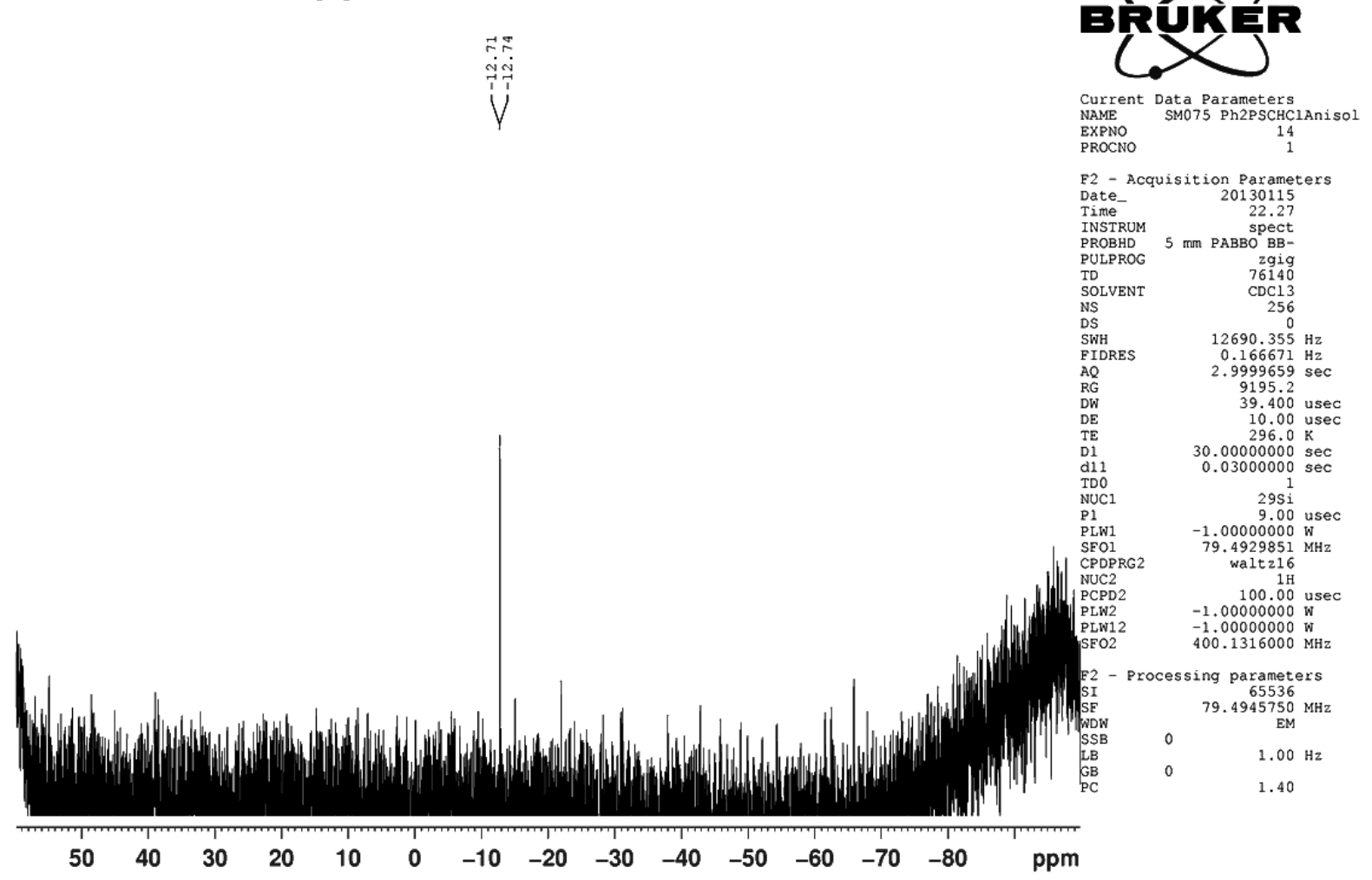

Figure $\mathbf{S 1 2} .{ }^{29} \mathrm{Si}\left\{{ }^{1} \mathrm{H}\right\}$ NMR spectrum of silane $\mathbf{1 f}$. 
SM095C orangene Kristalle Rest AP31CPD C6D 6 \{D: \Topspin21\} User 48
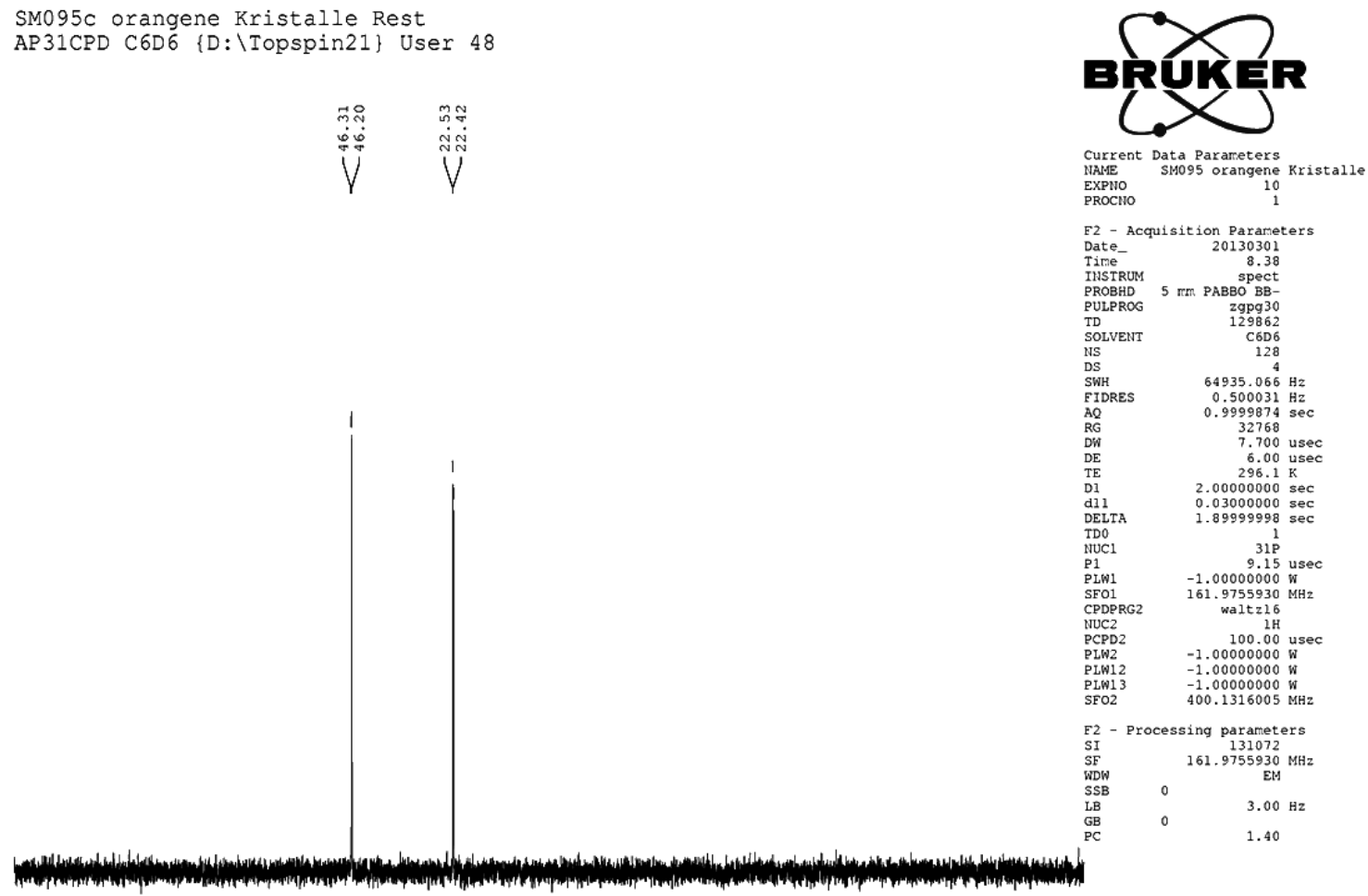

PROBHD
PULPROG

SISENT

SWH

64935.066 H

$0.500031 \mathrm{~Hz}$
$0.9999874 \mathrm{sec}$

32768 usec
7.7000 usec
6.00 usec
$296.1 \mathrm{~K}$
$2.0000000 \mathrm{sec}$

$\begin{array}{ll}2.00000000 \mathrm{sec} \\ 0.0300000 \mathrm{sec} \\ 1 & 1.89999998 \mathrm{sec}\end{array}$

$31 \mathrm{P}$
9.15
usec

$-1.00000000 \mathrm{~W}$
01.9755930

10

$\begin{array}{ll}2 & -1.00000000 \mathrm{~W} \\ 2 & -1.0000000 \mathrm{~W}\end{array}$

$400.1316005 \mathrm{M}$

2
SI
SE Processing parameters
131072
$161.9755930 \mathrm{MHz}$

$\begin{array}{lll}S S B & 0 & 3.00 \mathrm{~Hz}\end{array}$

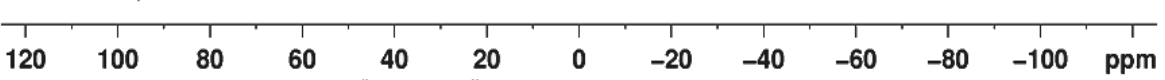

ㅎํ \%

SM095g Charakterisierung orangene Kristalle APROTON CD2C12 \{D: \Topspin21\} User 47

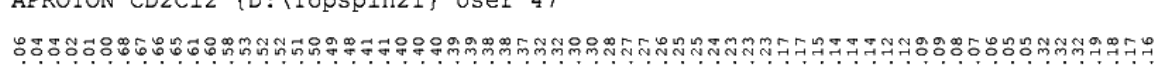

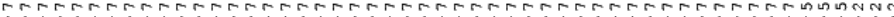

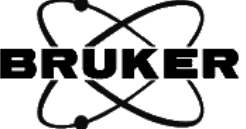
Current Data Parameters
MAME
EXPNO
SM095 ox. Add. SiPh $3+C 1$
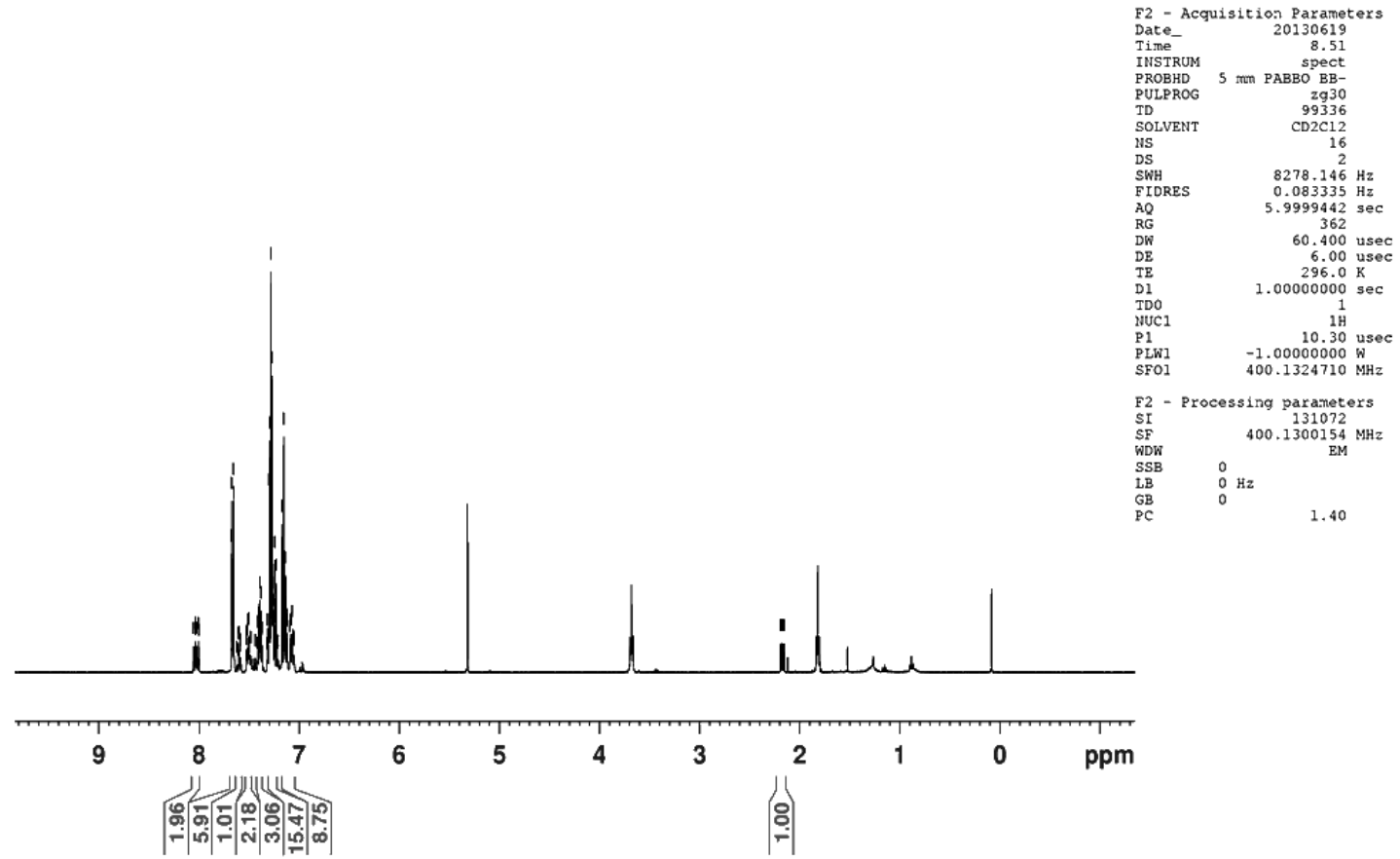

Figure S13. ${ }^{31} \mathrm{P}\left\{{ }^{1} \mathrm{H}\right\}$ NMR and ${ }^{1} \mathrm{H}$ NMR spectra of palladium complex 2a. 

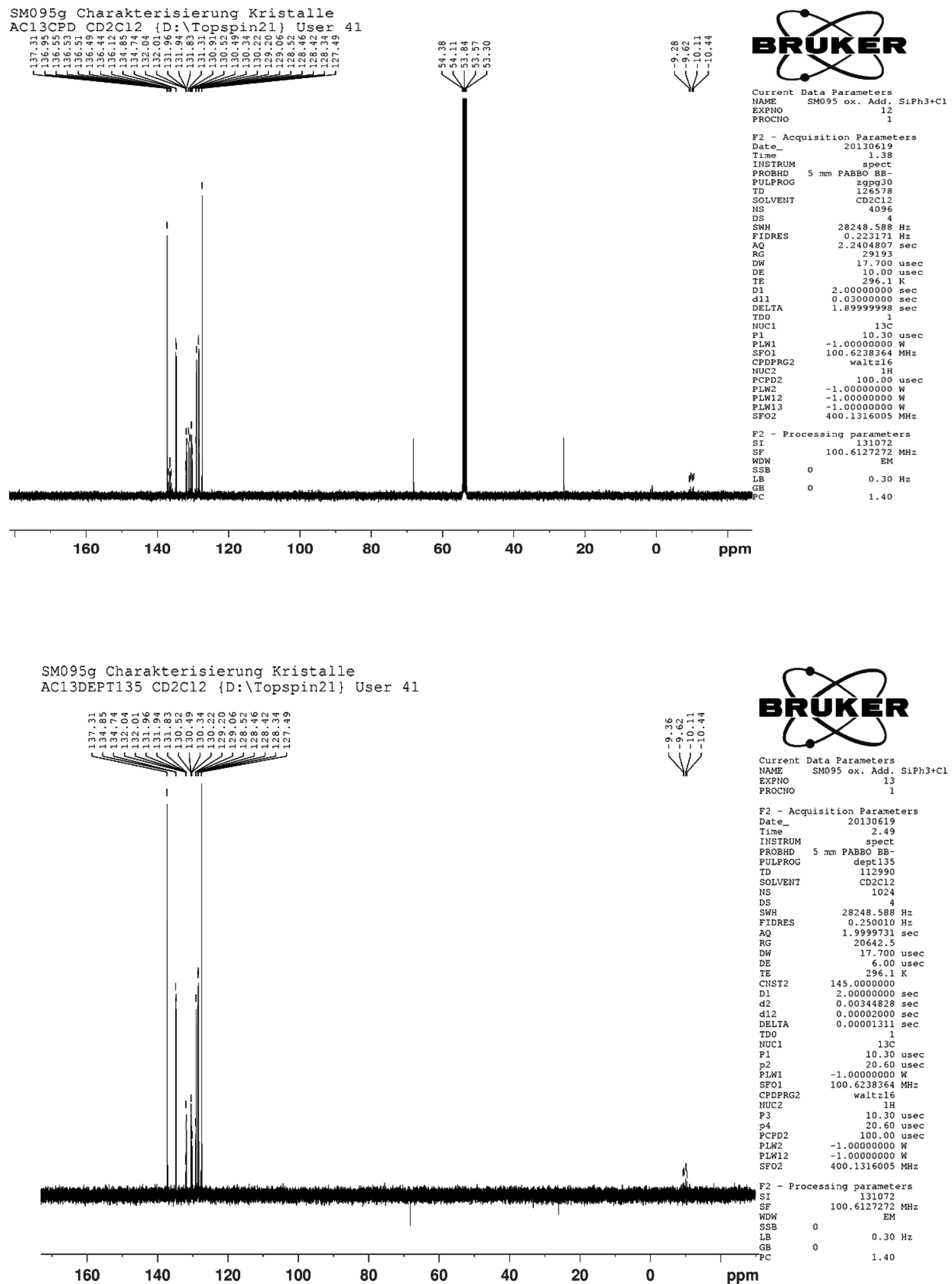

Figure S14. ${ }^{13} \mathrm{C}\left\{{ }^{1} \mathrm{H}\right\}$ NMR and ${ }^{13} \mathrm{C}\left\{{ }^{1} \mathrm{H}\right\}$-DEPT135 NMR spectra of palladium complex $2 \mathrm{a}$. 


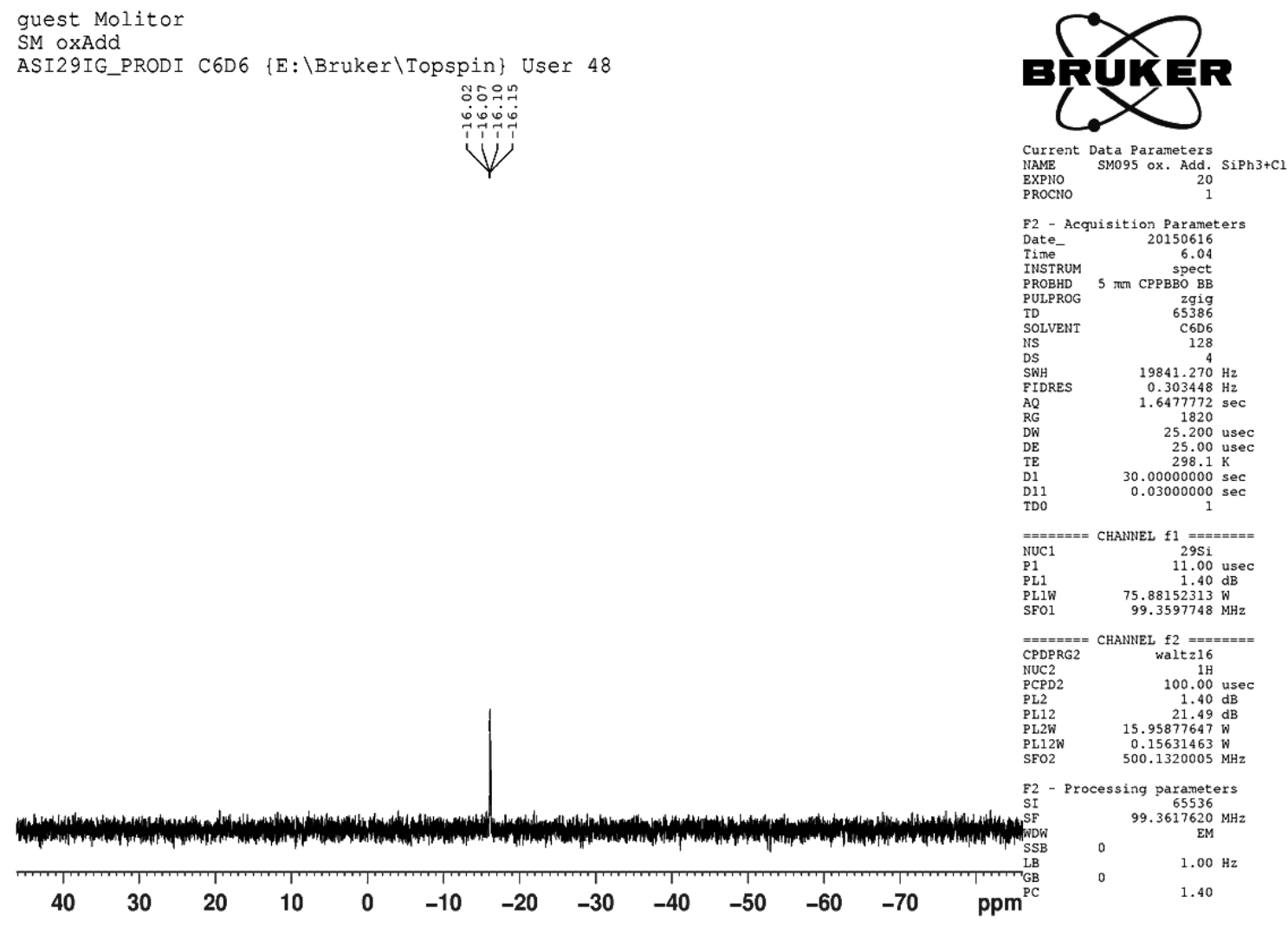

Figure S15. ${ }^{29} \mathrm{Si}\left\{{ }^{1} \mathrm{H}\right\}$ NMR spectrum of palladium complex $2 \mathbf{a}$. 


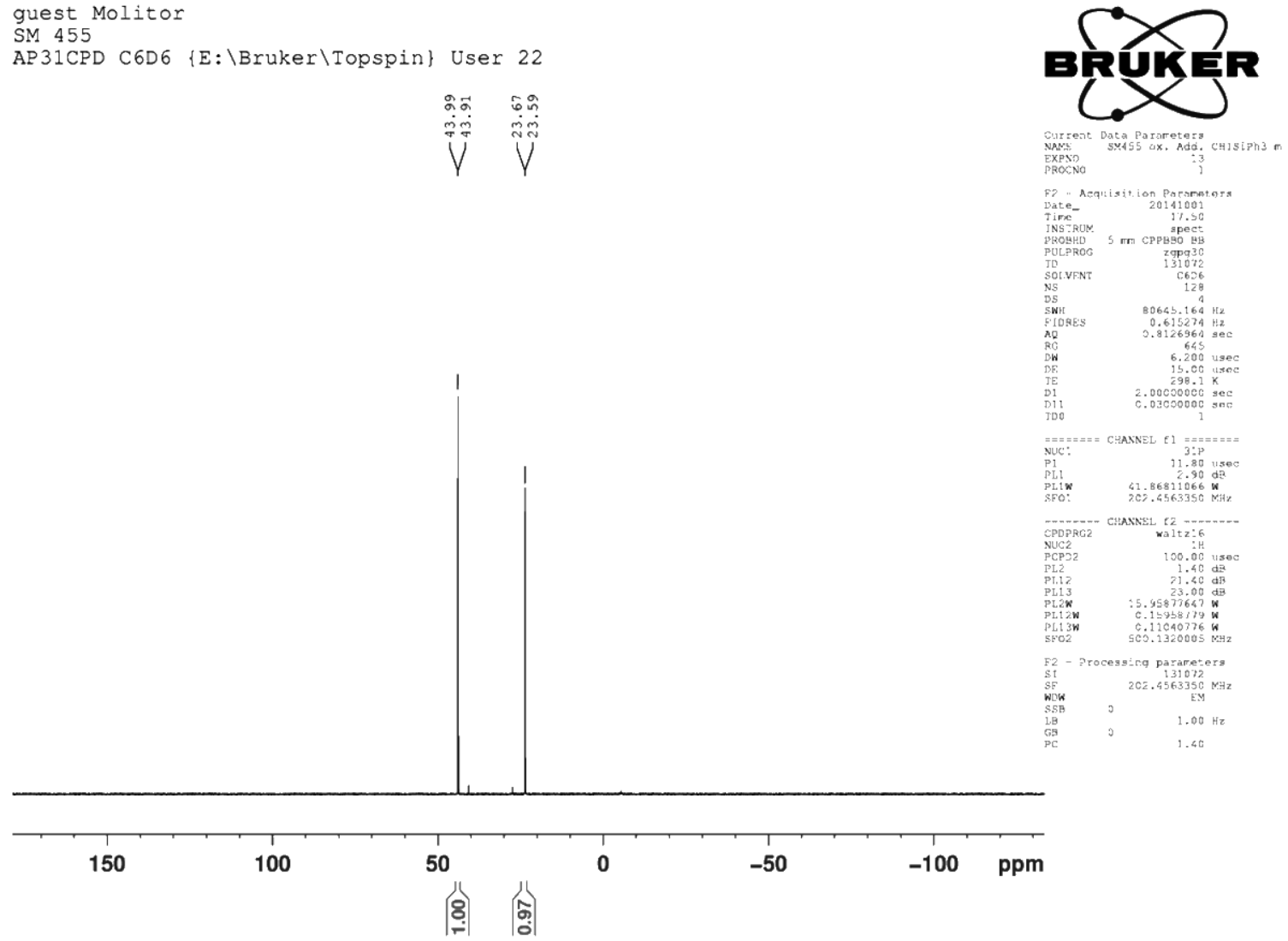

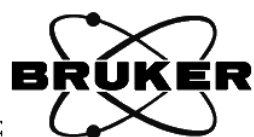

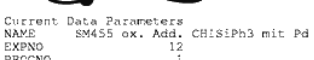

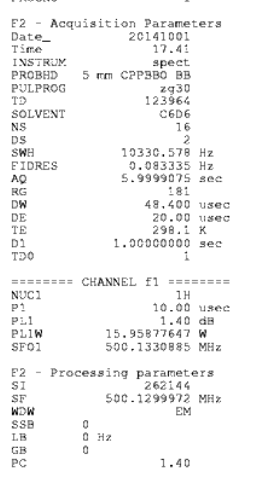

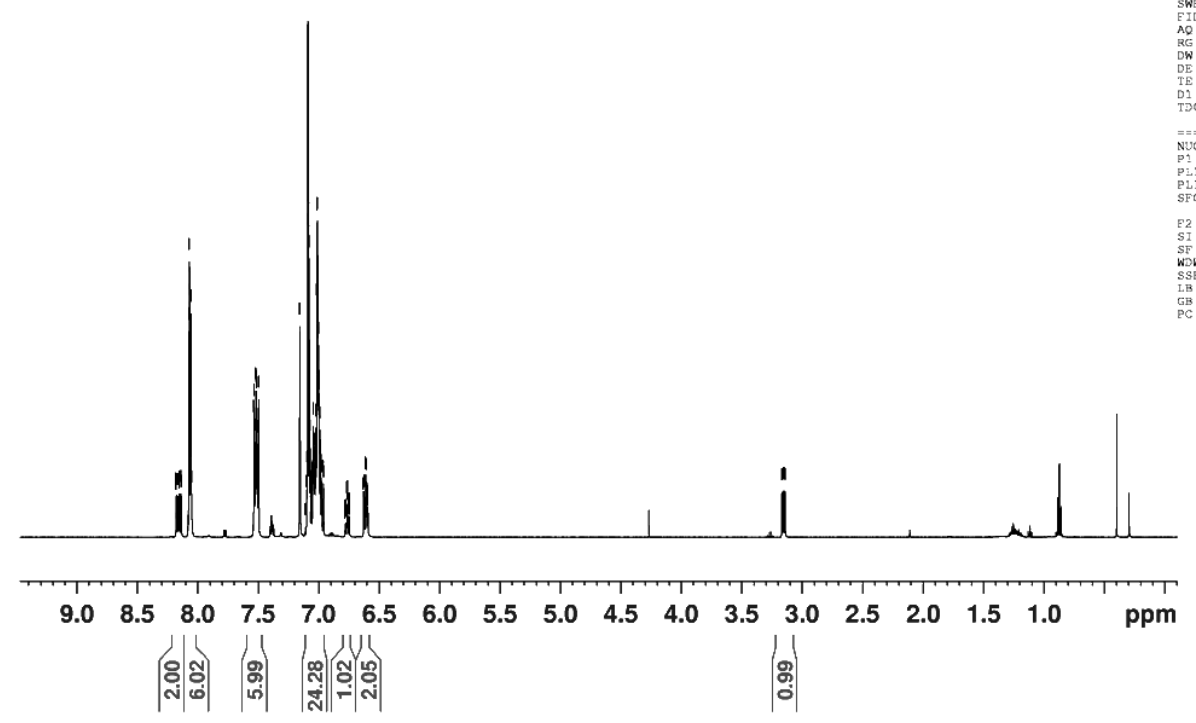

Figure S16. ${ }^{31} \mathrm{P}\left\{{ }^{1} \mathrm{H}\right\}$ NMR and ${ }^{1} \mathrm{H}$ NMR spectra of palladium complex $\mathbf{2 b}$. 


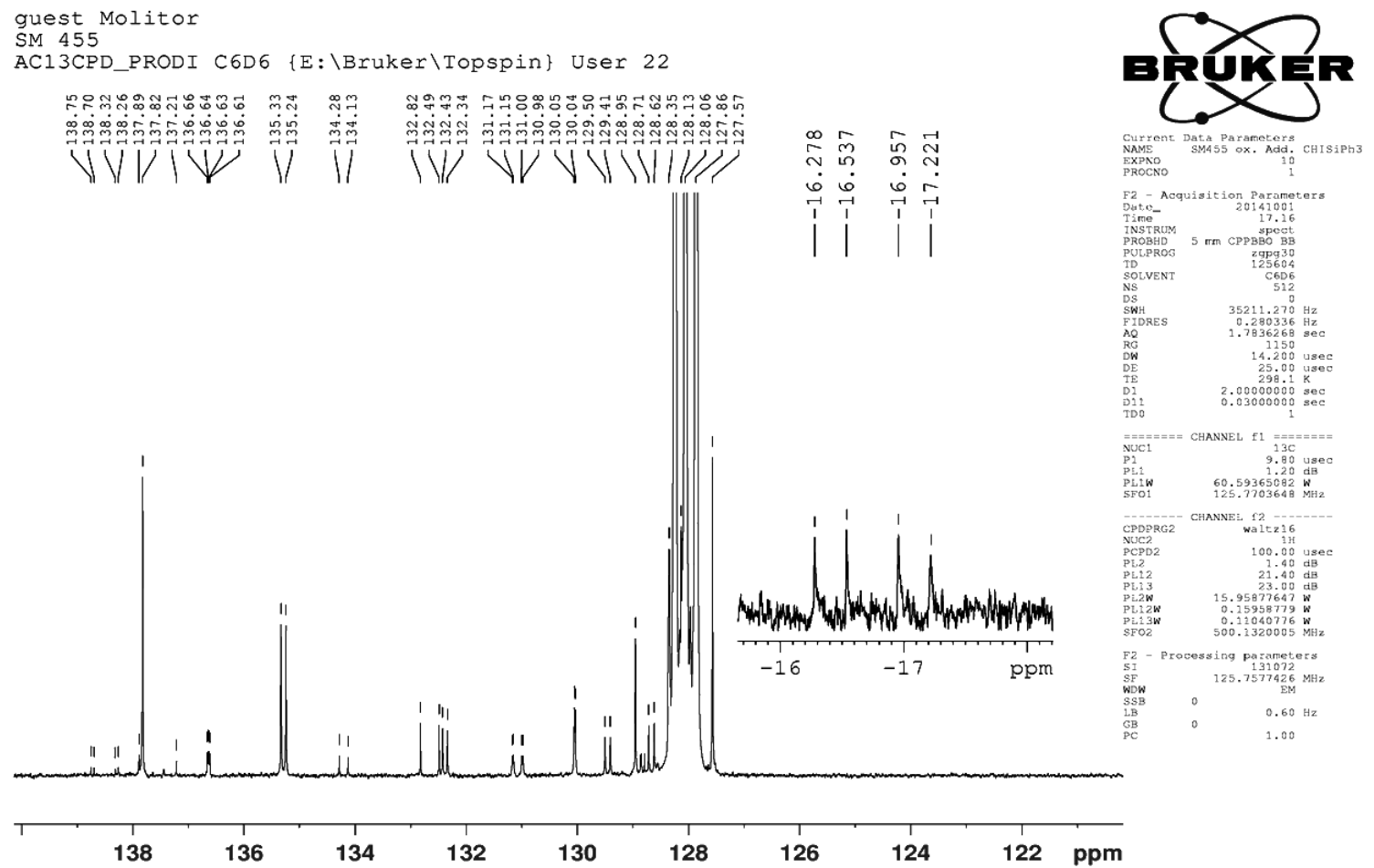

guest Molitor

SM 455

AC13DEPT135_PRODI C6D6 \{E:\Bruker\Topspin\} User 22

N $\quad$ mp

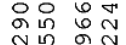

$\dot{\varphi} \dot{\varphi} \dot{r}$

$\begin{array}{ccc}0 & 0 \\ -1 & -1 & -1\end{array}$

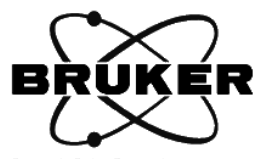

Current Data Parameters
NAME
EXPNO
SKG5 Ox. Add. CHISiph3 mit Pd
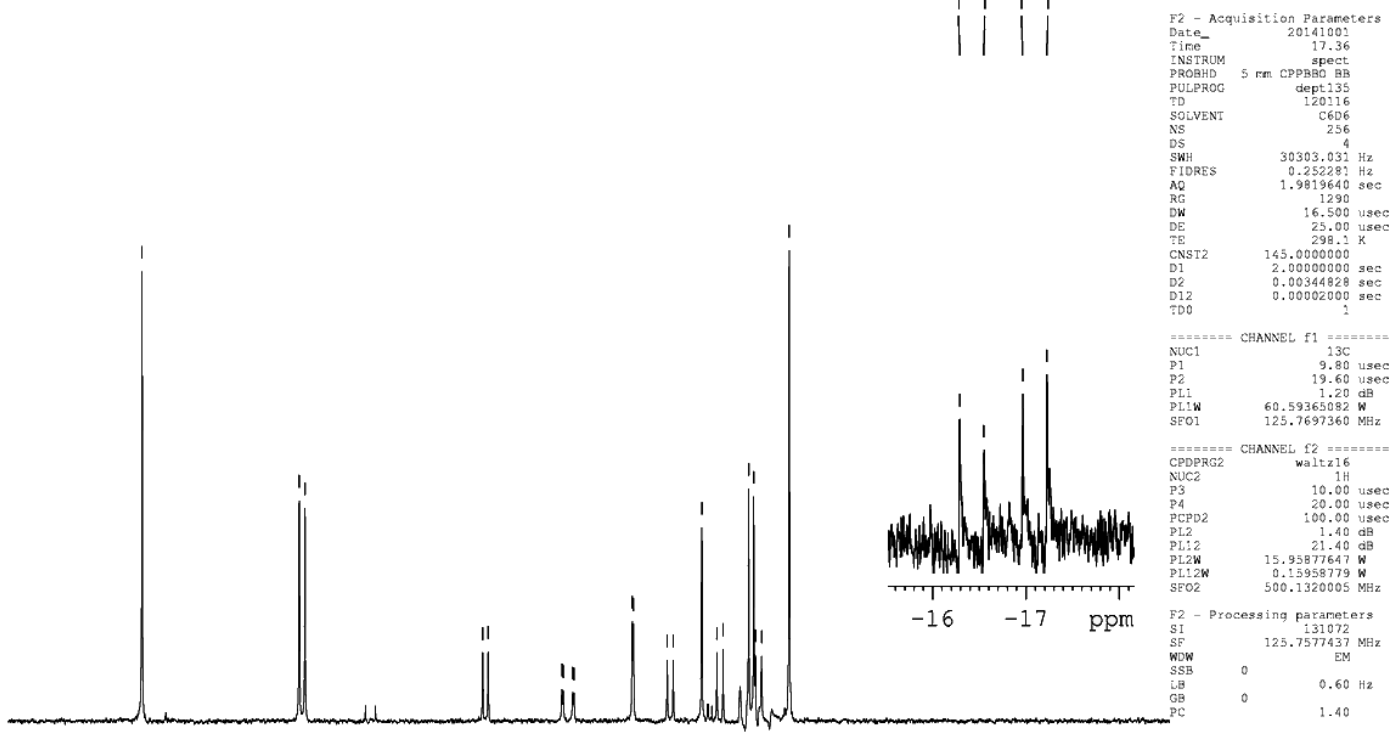

$\begin{array}{llllllllllllllllll}139 & 138 & 137 & 136 & 135 & 134 & 133 & 132 & 131 & 130 & 129 & 128 & 127 & 126 & 125 & 124 & 123 & \mathrm{ppm}\end{array}$

Figure S17. ${ }^{13} \mathrm{C}\left\{{ }^{1} \mathrm{H}\right\}$ NMR and ${ }^{13} \mathrm{C}\left\{{ }^{1} \mathrm{H}\right\}$-DEPT135 NMR spectra of palladium complex $\mathbf{2 b}$. 
guest Molitor
SM 455
ASI29IG_PRODI C6D6 \{E: \Bruker\Topspin $\}$ User 22
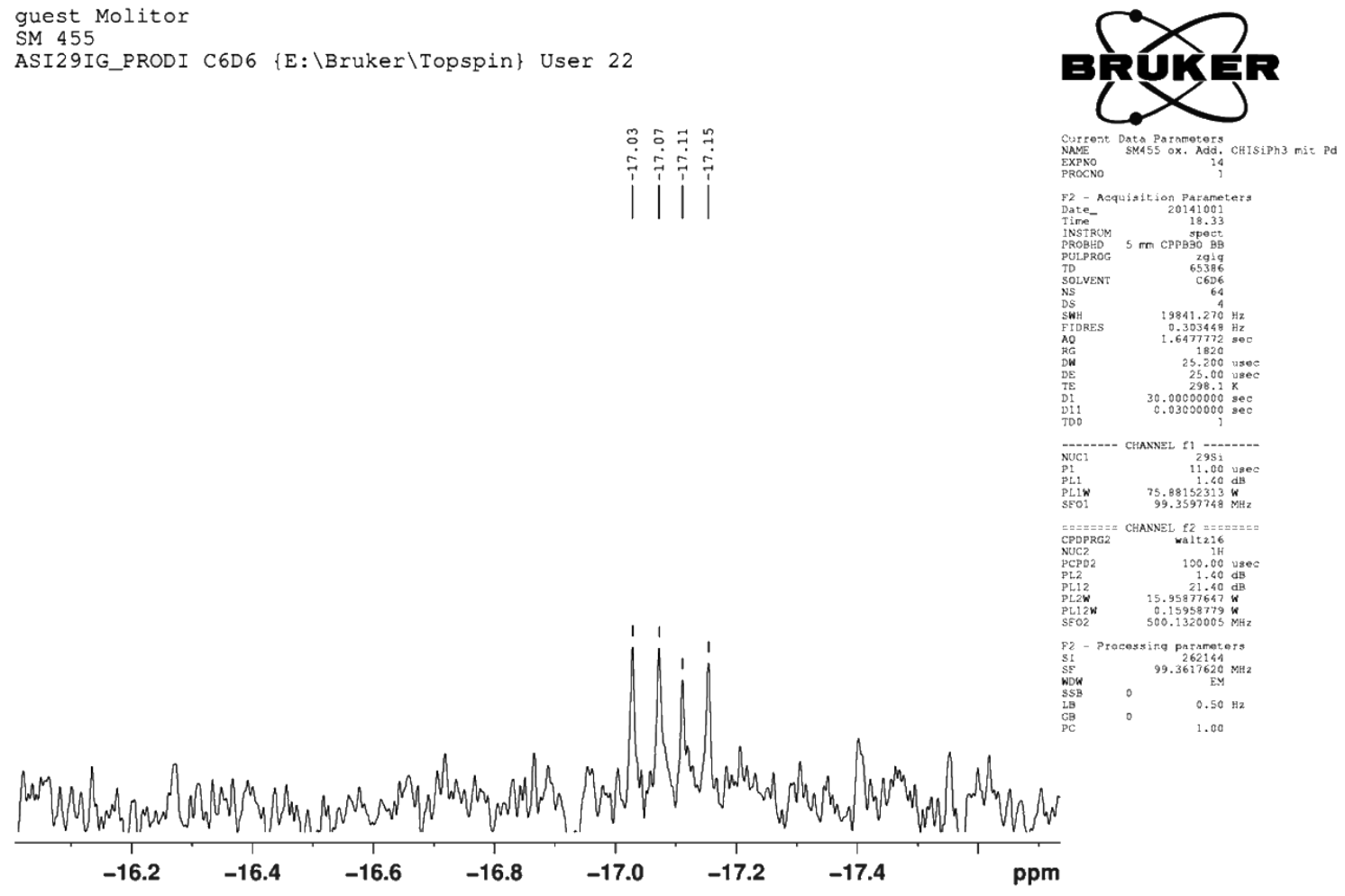

Figure S18. ${ }^{29} \mathrm{Si}\left\{{ }^{1} \mathrm{H}\right\}$ NMR spectrum of palladium complex $\mathbf{2 b}$. 


\section{guest Molitor}

SM 452

AP31CPD C6D6 \{E: \Bruker\Topspin\} User 21

Vู
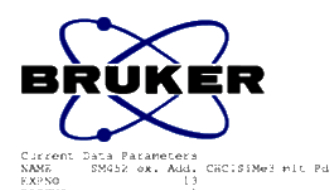

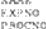
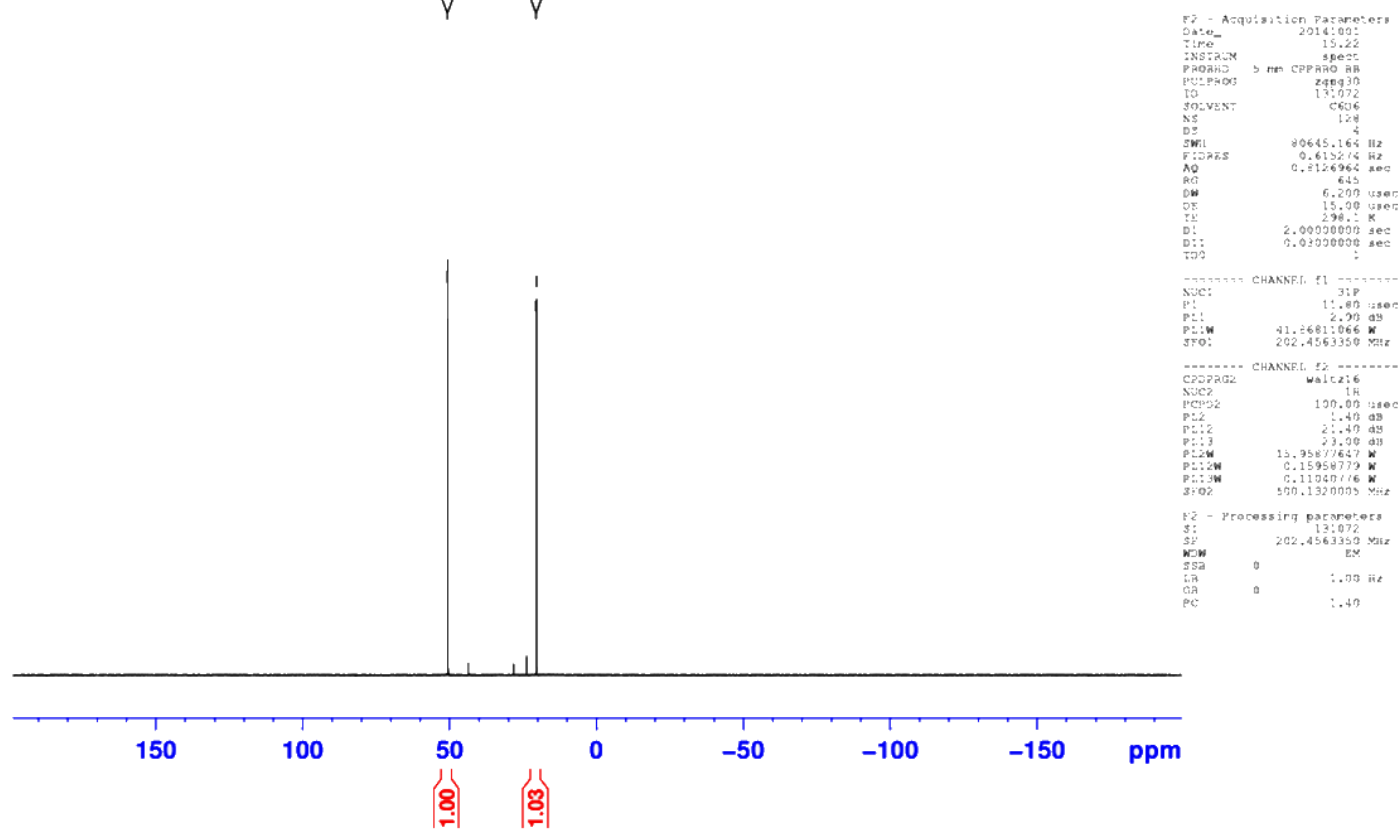

guest Molitor

SM4 52

APROTON16_PRODI C6D6 \{E:\Bruker\Topspin\} User 21

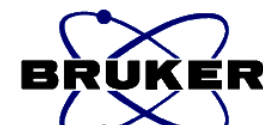

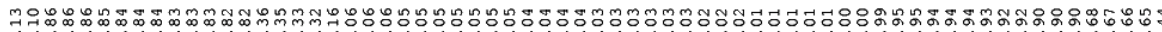

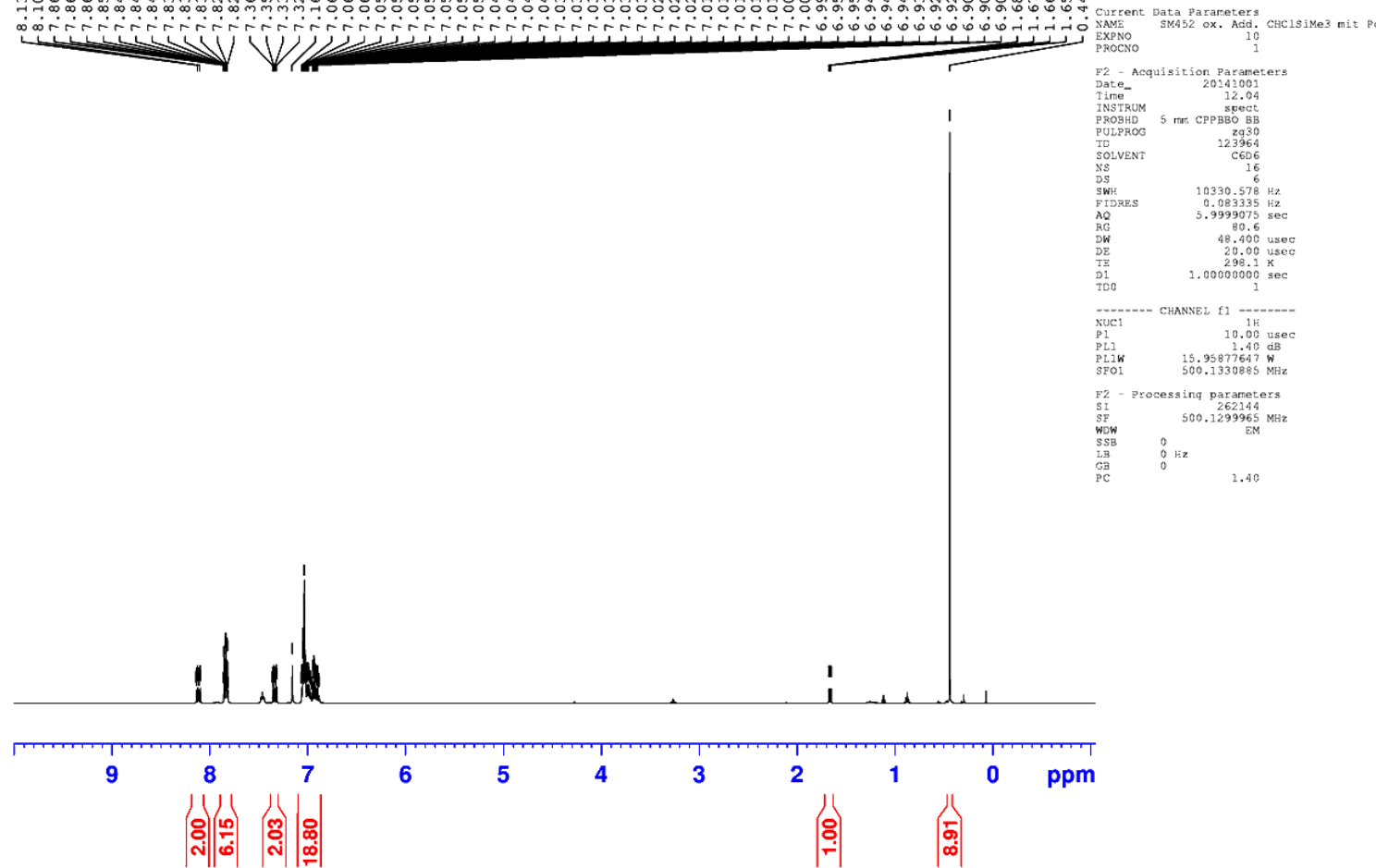

Figure S19. ${ }^{31} \mathrm{P}\left\{{ }^{1} \mathrm{H}\right\}$ NMR and ${ }^{1} \mathrm{H}$ NMR spectra of palladium complex 2c. 
guest Molitor

SM4 52

AC13CPD_PRODI C6D 6 \{E: \Bruker \Topspin\} User 21

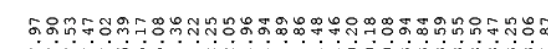

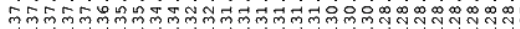

NIV

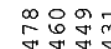

ํํำ

mंmंm

वं वं कि

trun
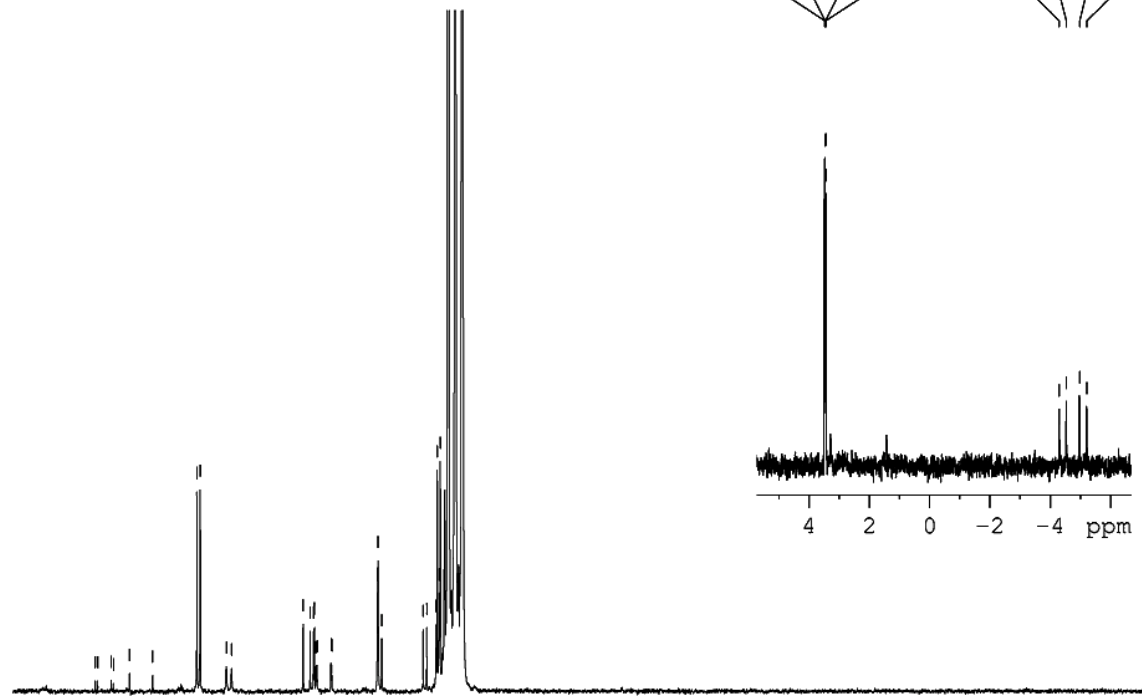

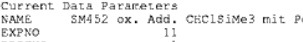

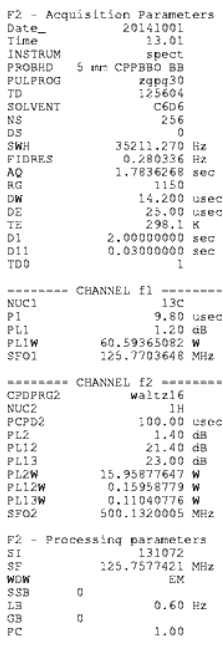

135

130

125

120

115

ppm

guest Molitor

SM 452

AC13DEPT135_PRODI C6D6 \{E:\Bruker\Topspin\} User 21
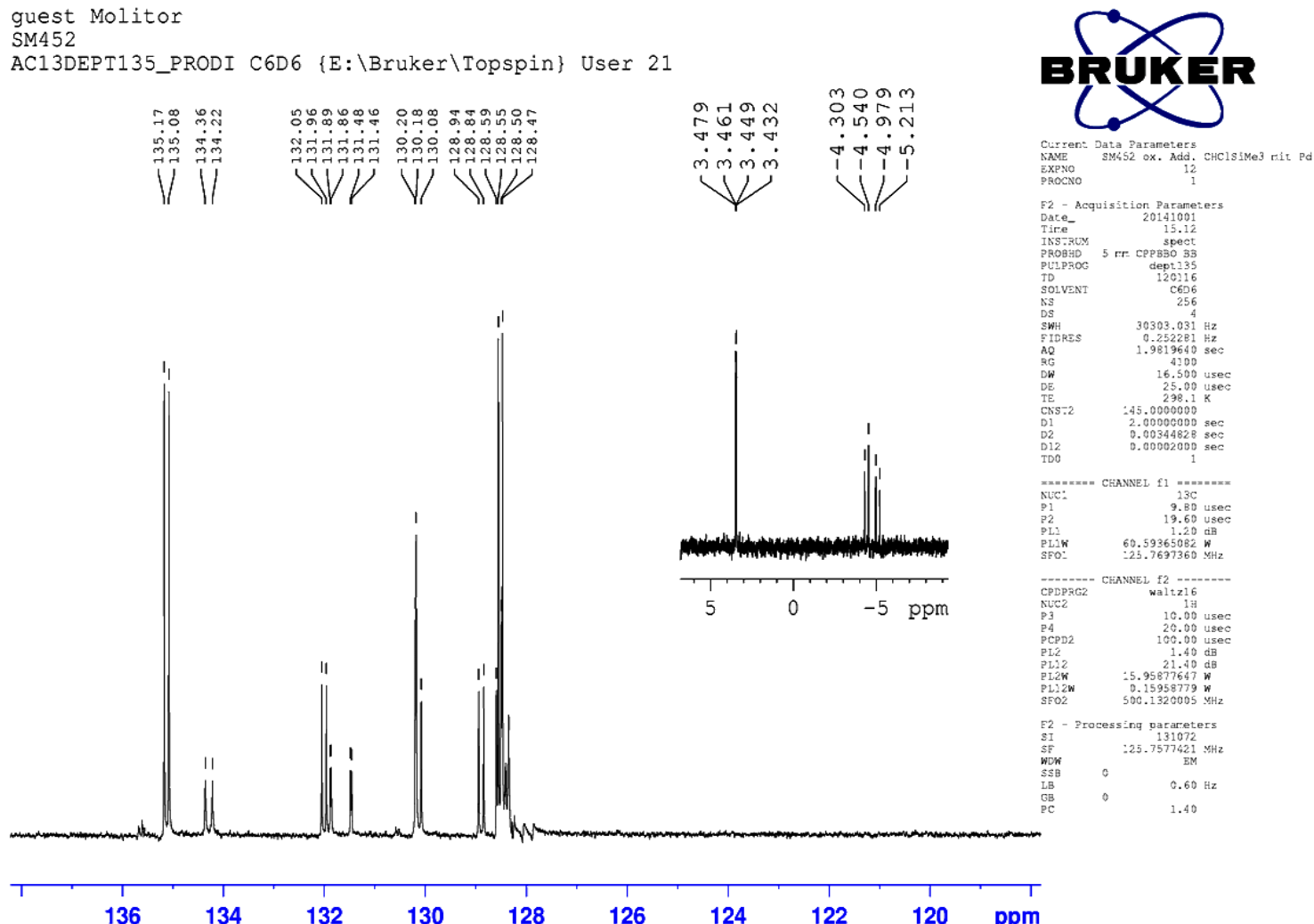

Figure S20. ${ }^{13} \mathrm{C}\left\{{ }^{1} \mathrm{H}\right\}$ NMR and ${ }^{13} \mathrm{C}\left\{{ }^{1} \mathrm{H}\right\}$-DEPT135 NMR spectra of palladium complex 2c. 


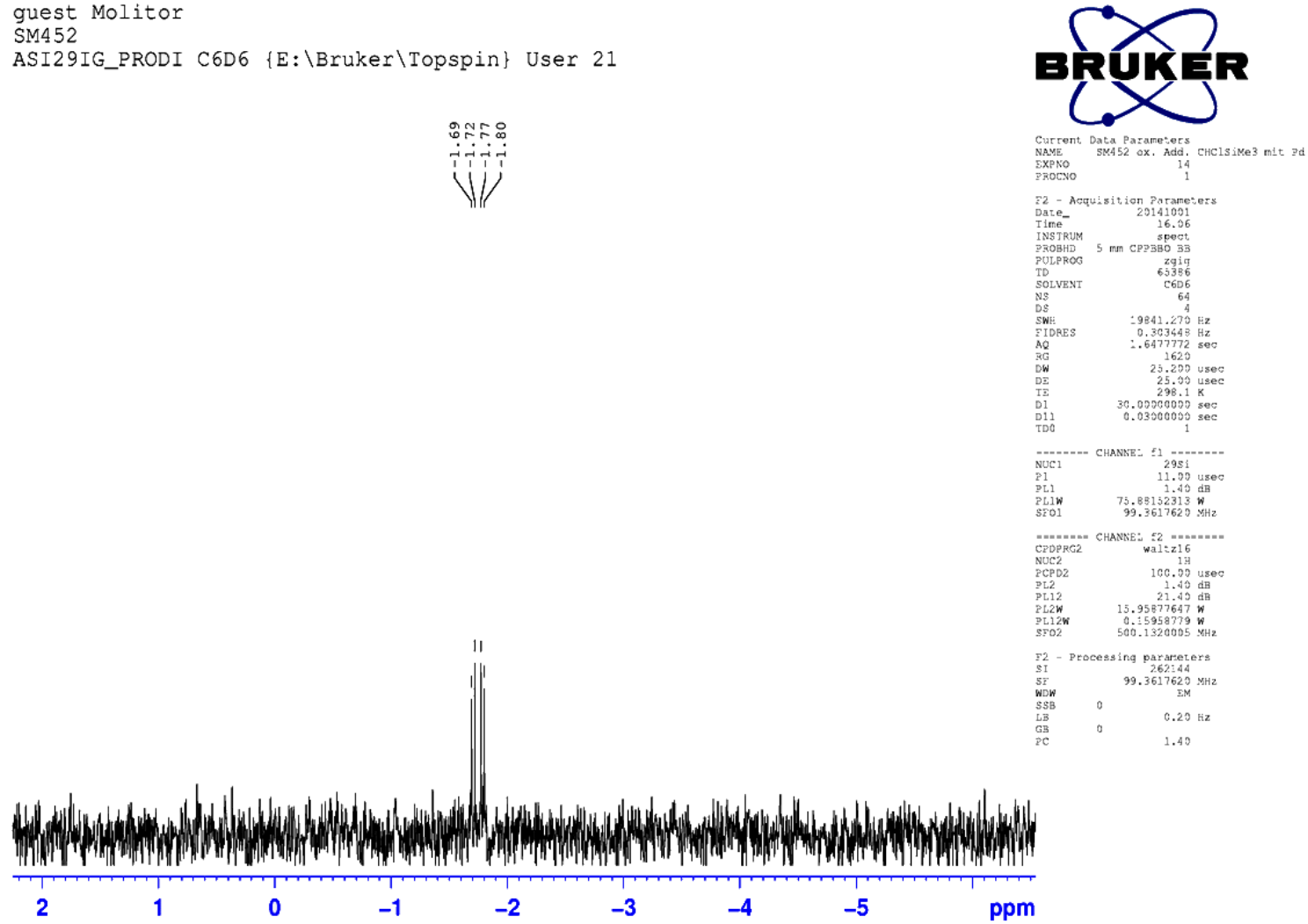

Figure $\mathbf{S 2 1} .{ }^{29} \mathrm{Si}\left\{{ }^{1} \mathrm{H}\right\}$ NMR spectrum of palladium complex $\mathbf{2 c}$. 
guest Molitor

SM 473

AP31CPD C6D6 \{E:\Bruker\Topspin\} User 33
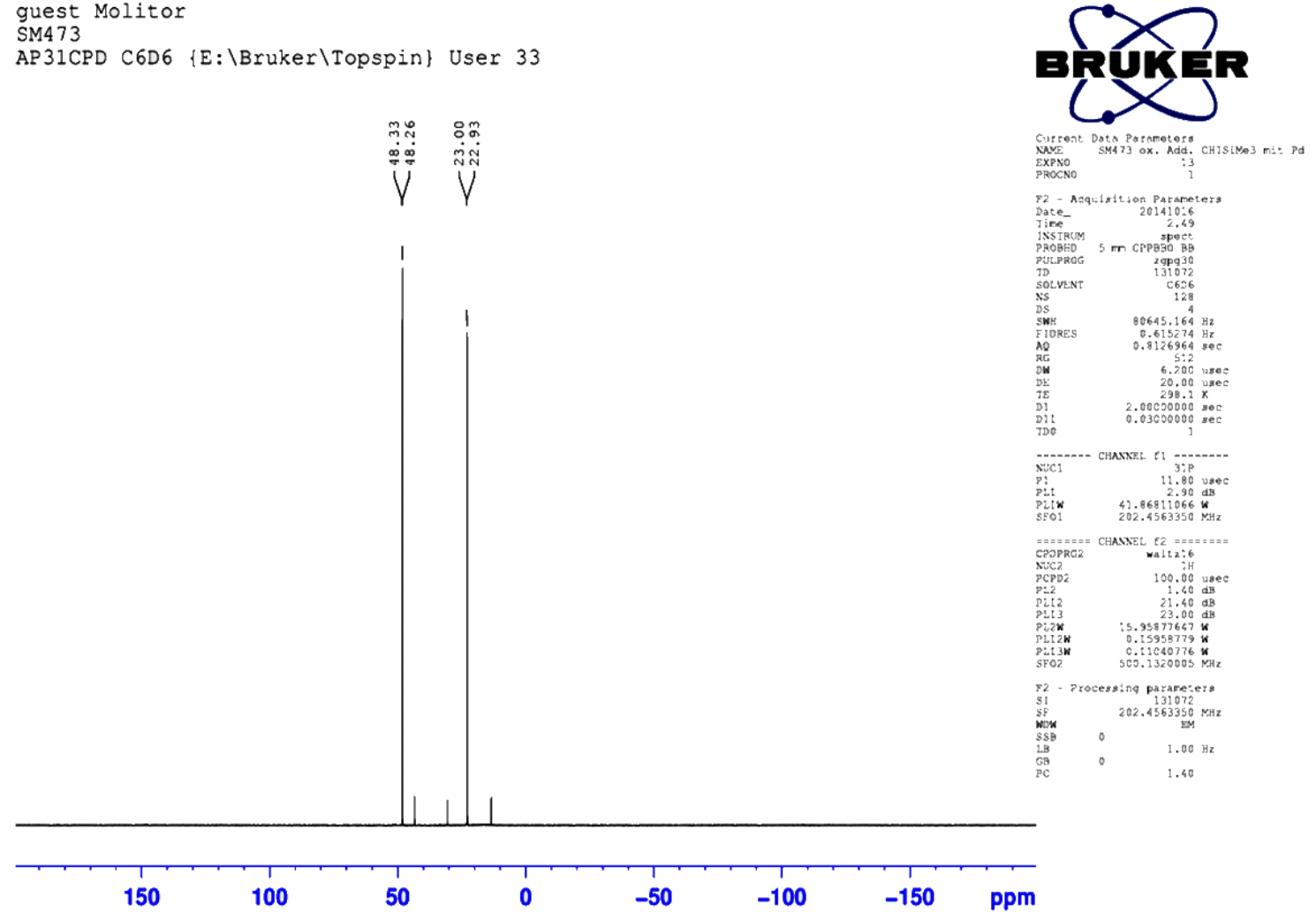

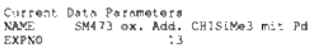

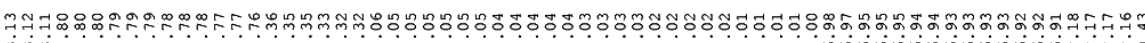

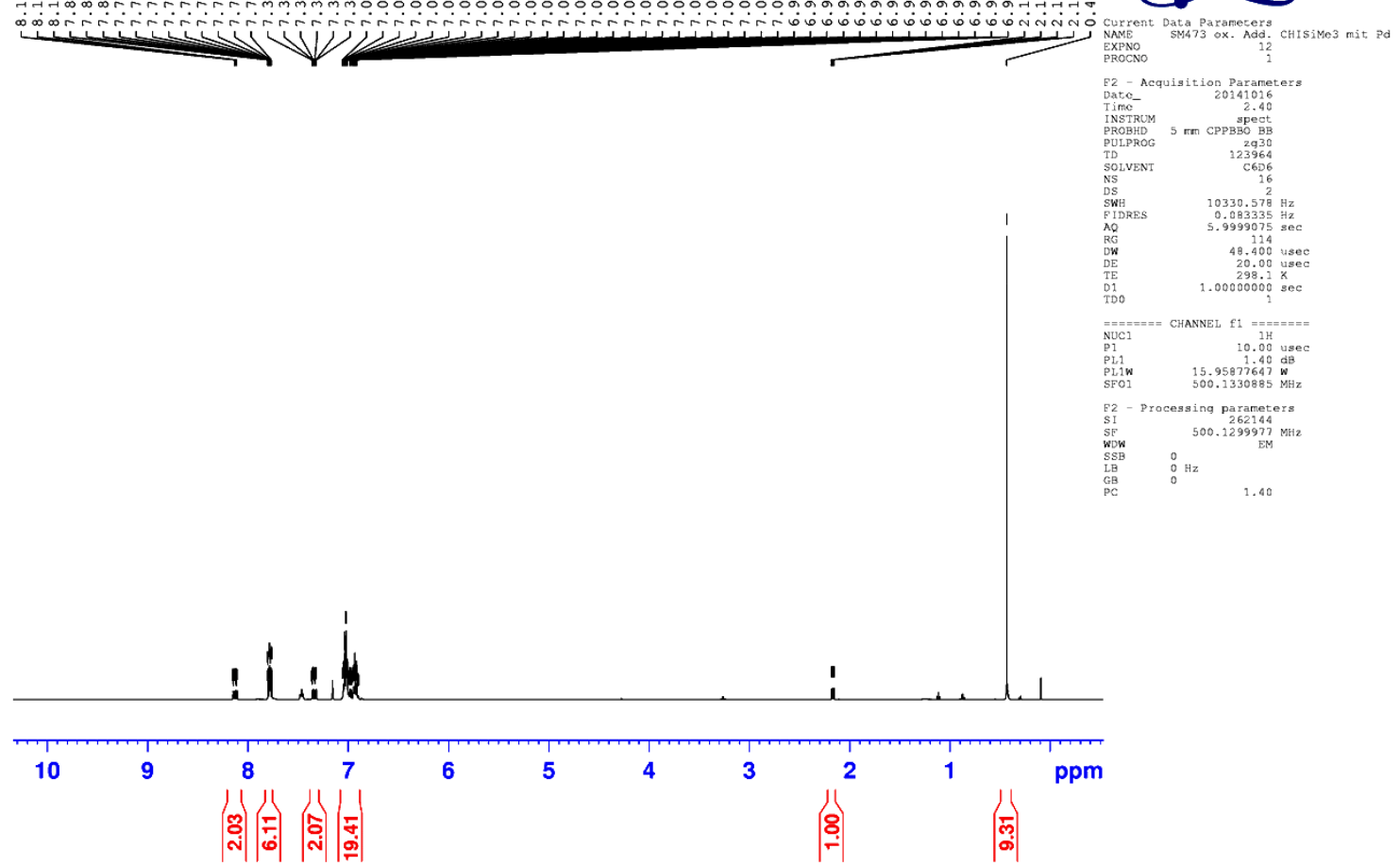

Figure S22. ${ }^{31} \mathrm{P}\left\{{ }^{1} \mathrm{H}\right\}$ NMR and ${ }^{1} \mathrm{H}$ NMR spectra of palladium complex $2 \mathbf{d}$. 

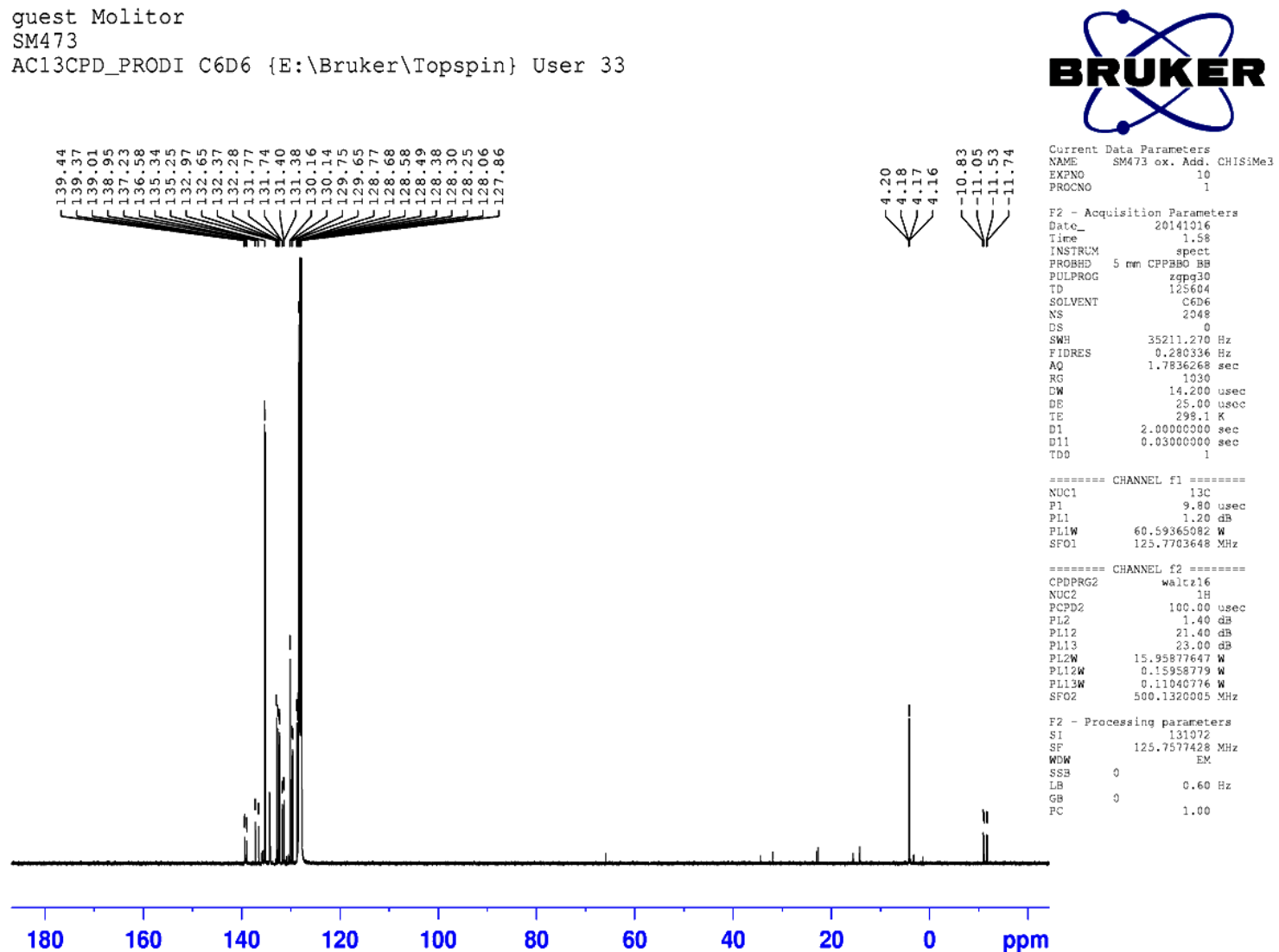

guest Molitor

SM 473

AC13DEPT135_PRODI C6D6 \{E: \Bruker\Topspin\} User 33

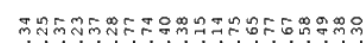

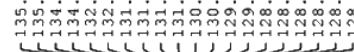
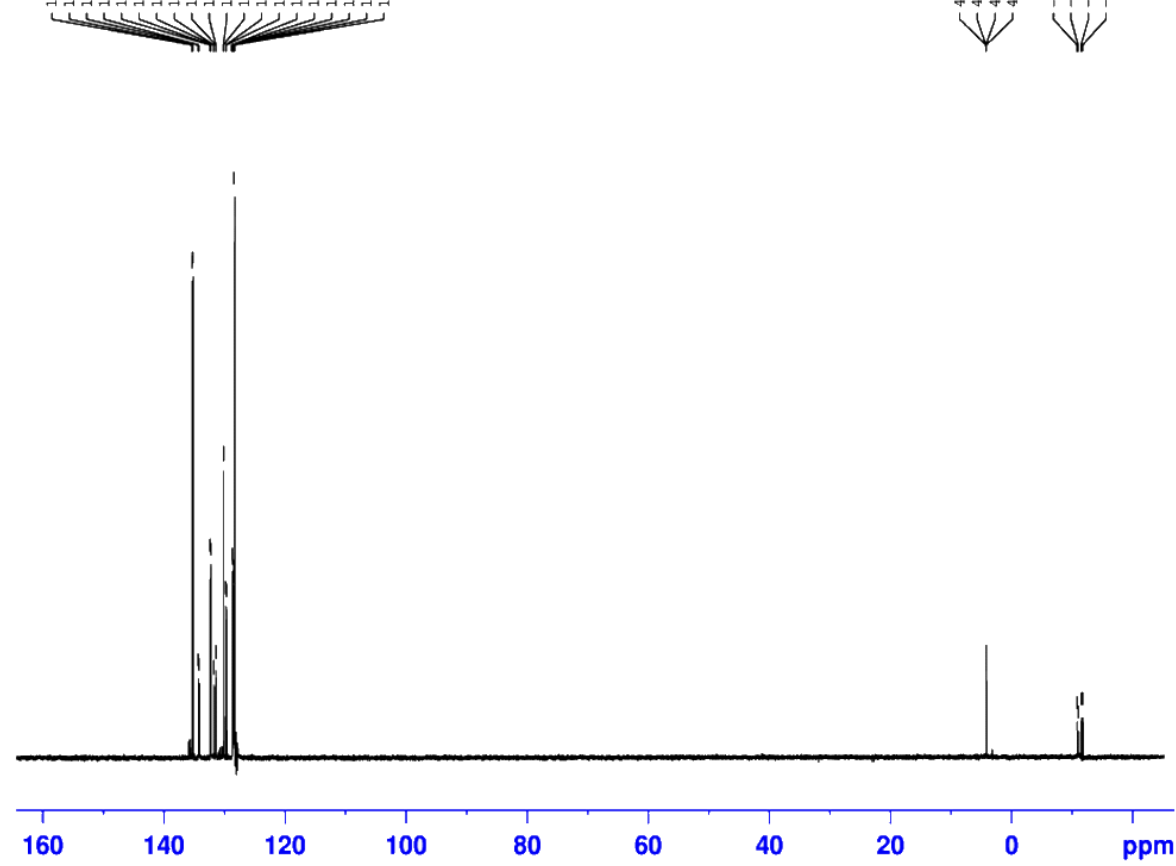

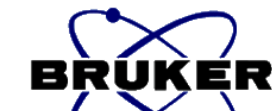

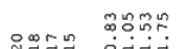

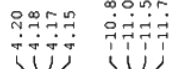

$\underbrace{}_{3 a t a p \text { parameters }}$

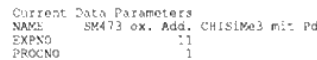

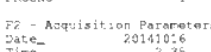

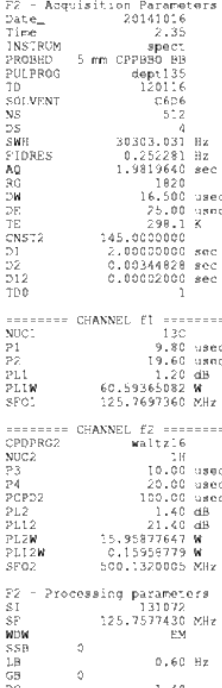

1.40

Figure S23. ${ }^{13} \mathrm{C}\left\{{ }^{1} \mathrm{H}\right\}$ NMR and ${ }^{13} \mathrm{C}\left\{{ }^{1} \mathrm{H}\right\}$-DEPT135 NMR spectra of palladium complex 2d. 


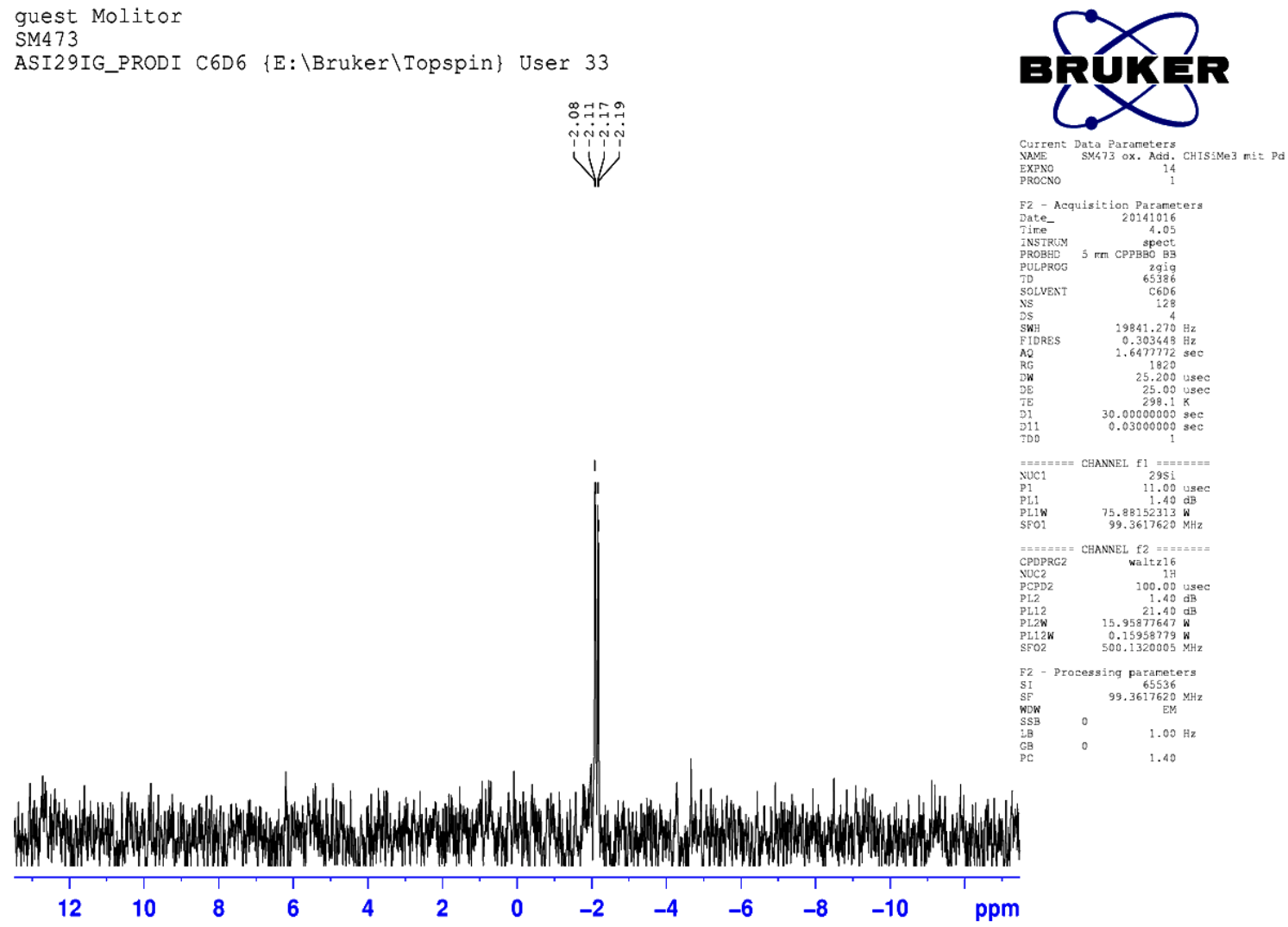

Figure S24. ${ }^{29} \mathrm{Si}\left\{{ }^{1} \mathrm{H}\right\}$ NMR spectrum of palladium complex 2 d. 


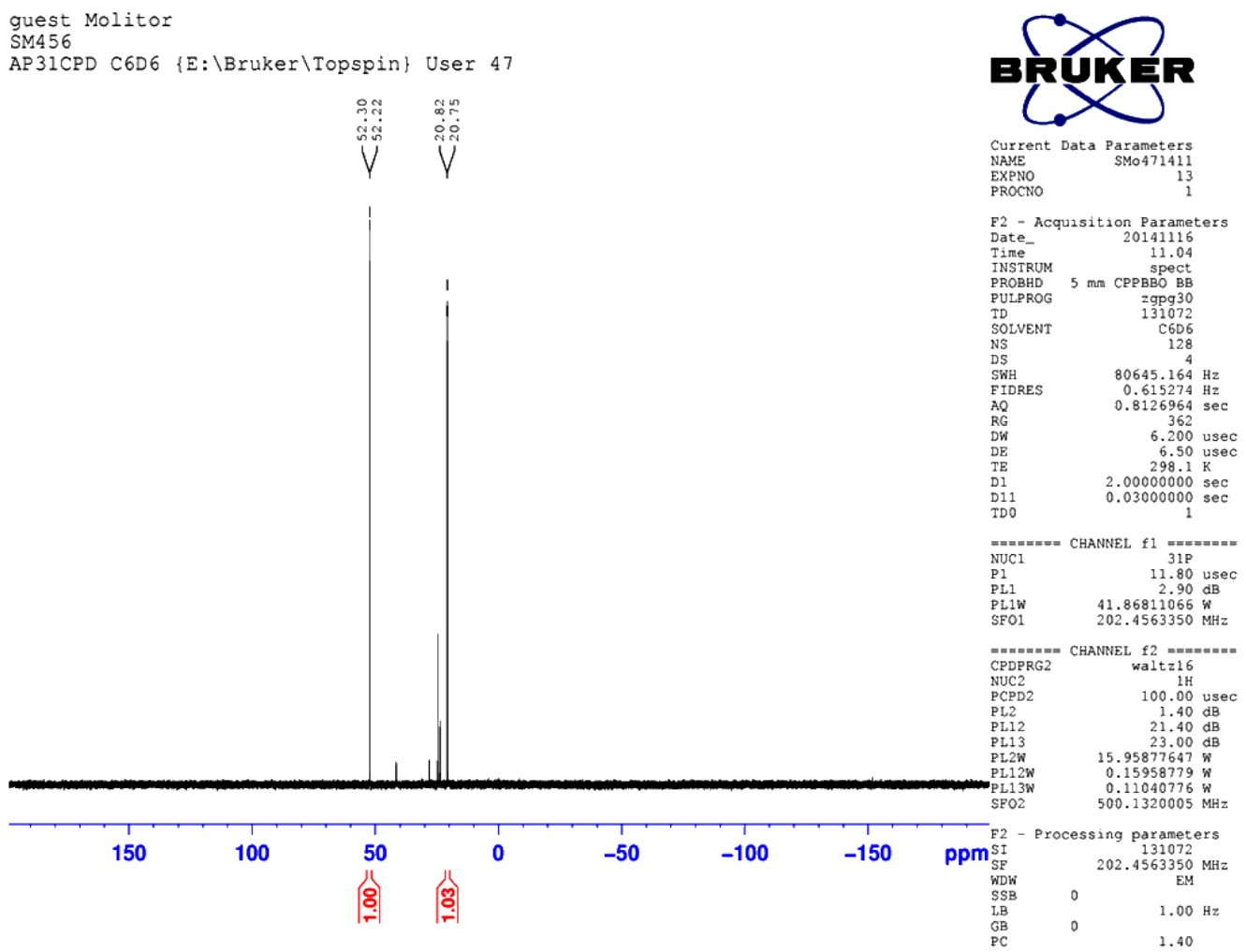

guest Molitor

APROTON16_PRODI C6D6 \{E:\Bruker\Topspin\} User 47

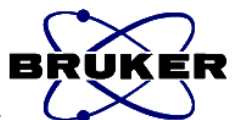

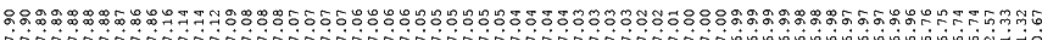

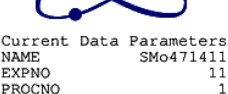

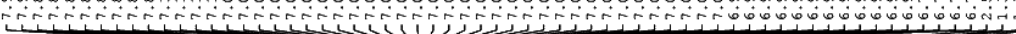
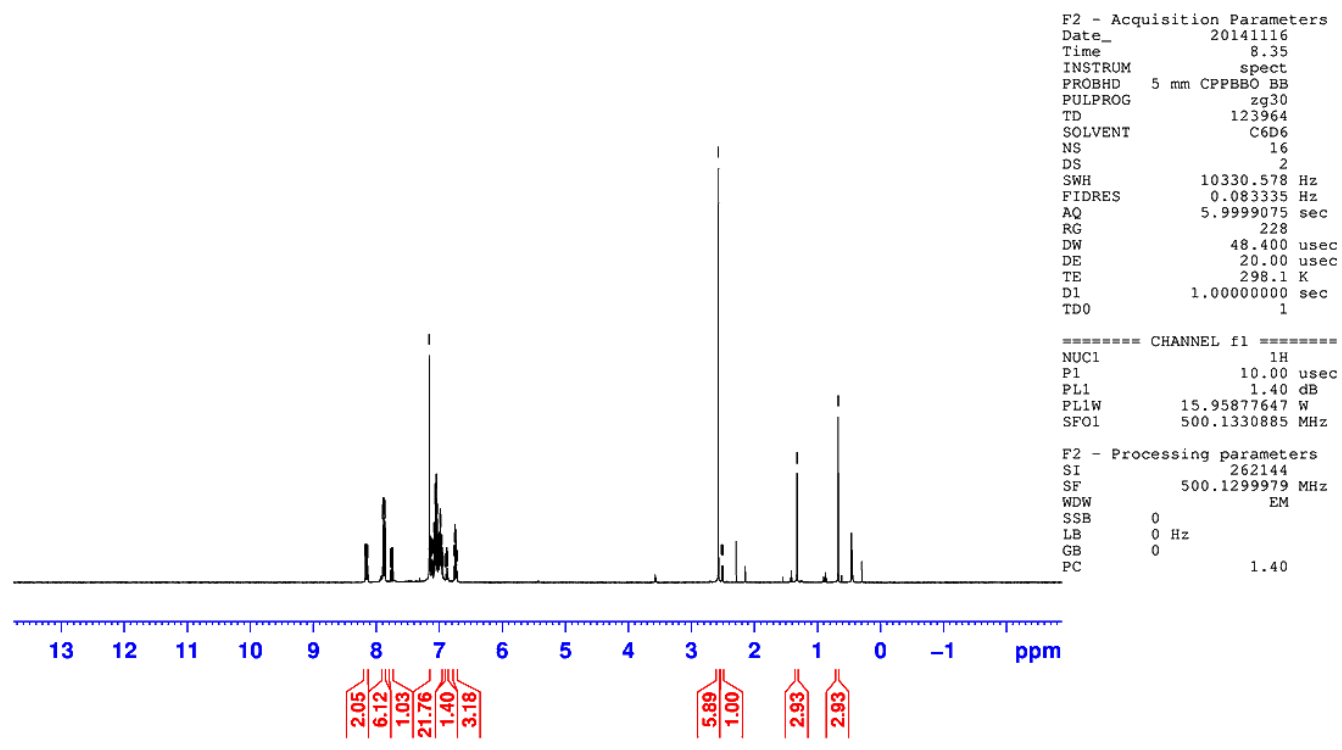

Figure S25. ${ }^{31} \mathrm{P}\left\{{ }^{1} \mathrm{H}\right\}$ NMR and ${ }^{1} \mathrm{H}$ NMR spectra of palladium complex $2 \mathbf{e}$. 

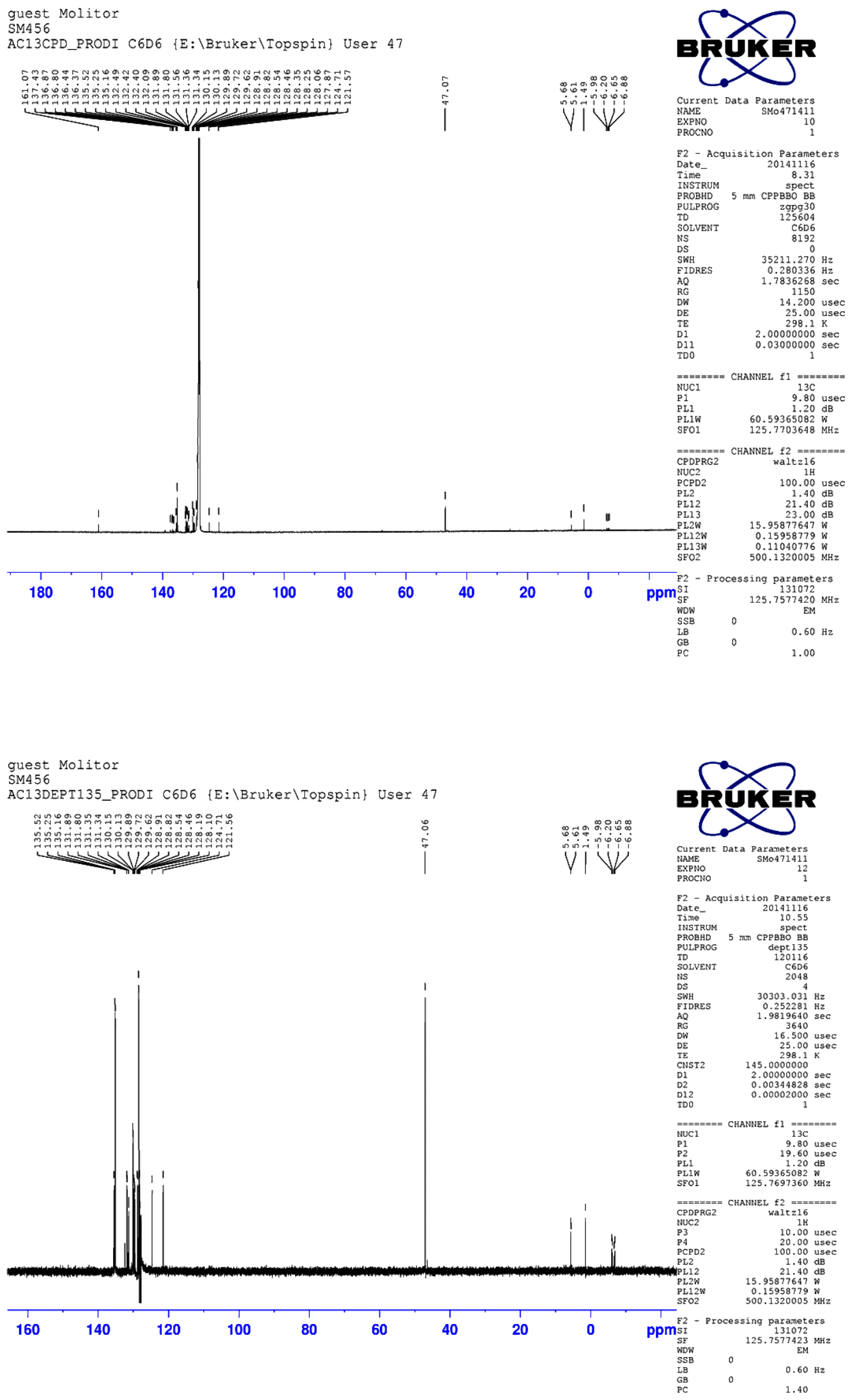

Figure S26. ${ }^{13} \mathrm{C}\left\{{ }^{1} \mathrm{H}\right\}$ NMR and ${ }^{13} \mathrm{C}\left\{{ }^{1} \mathrm{H}\right\}$-DEPT135 NMR spectra of palladium complex $2 \mathrm{e}$. 
guest Molitor

SM 456

ASI29IG_PRODI C6D6 \{E:\Bruker\Topspin\} User 47

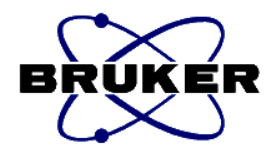

ํㅗㅇํㅇㅇํㅇ

$\begin{array}{lr}\text { Current Data } & \text { Parameters } \\ \text { NAME } & \text { SMO471411 } \\ \text { EXPNO } & 14 \\ \text { PROCNO } & 1\end{array}$

F2 - Acquisition Parameters

F2 - Acquisition Parame
Date_-
Time

spect
INSTRUM

PROBHD
PULPROG

$\begin{array}{lr}\text { TD } & \text { 2gig } \\ \text { TDOLVENT } & 65386 \\ \text { NS } & \text { C6D6 } \\ & 1024\end{array}$

$\begin{array}{lr}\text { NS } & 1024 \\ \text { DS } & 4 \\ \text { SWH } & 19841,270 \mathrm{~Hz} \\ \text { TIDPES } & 0.303448 \mathrm{~Hz}\end{array}$

$\begin{array}{ll}\text { FIDRES } & 0.303448 \mathrm{~Hz} \\ \text { AQ } & 1.6477772 \mathrm{sec}\end{array}$

$\begin{array}{lr}\text { AQ } & 1.6477772 \\ \text { RG } & 1820 \mathrm{sec} \\ \text { DW } & 25.200 \text { usec }\end{array}$

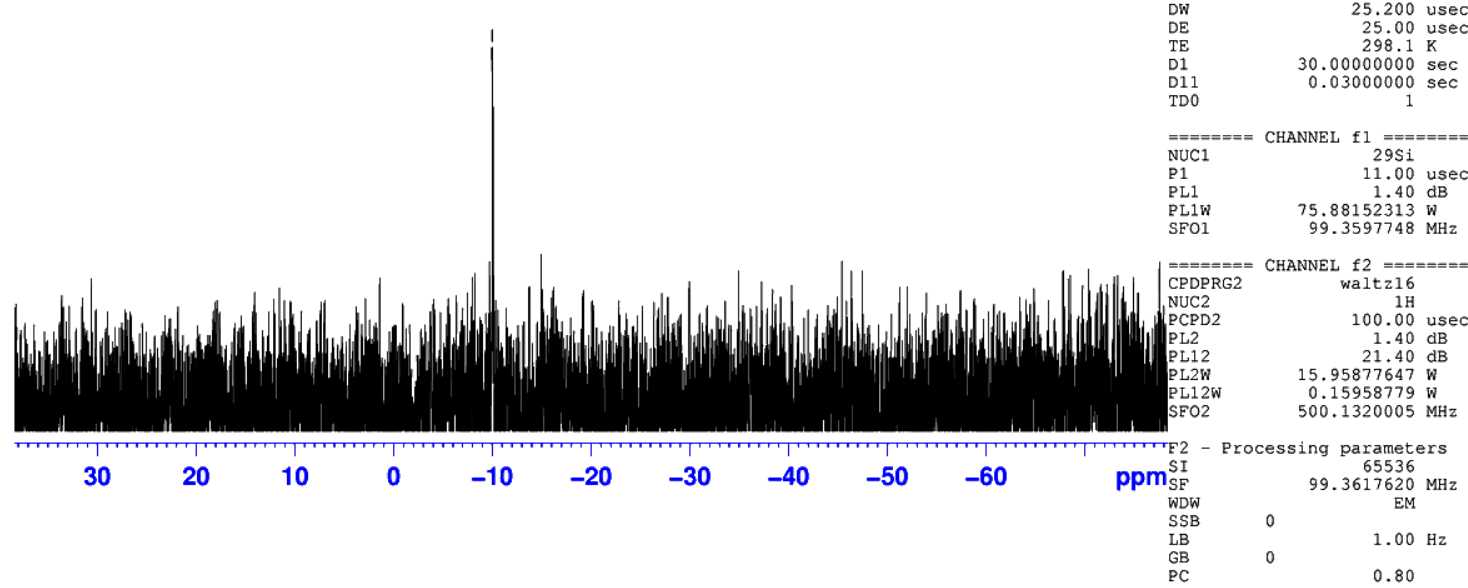

Figure S27. ${ }^{29} \mathrm{Si}\left\{{ }^{1} \mathrm{H}\right\}$ NMR spectrum of palladium complex $\mathbf{2 e}$. 
guest Molitor

SM504

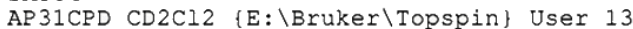

ที่

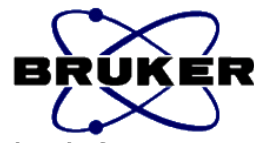

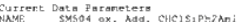

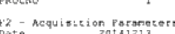
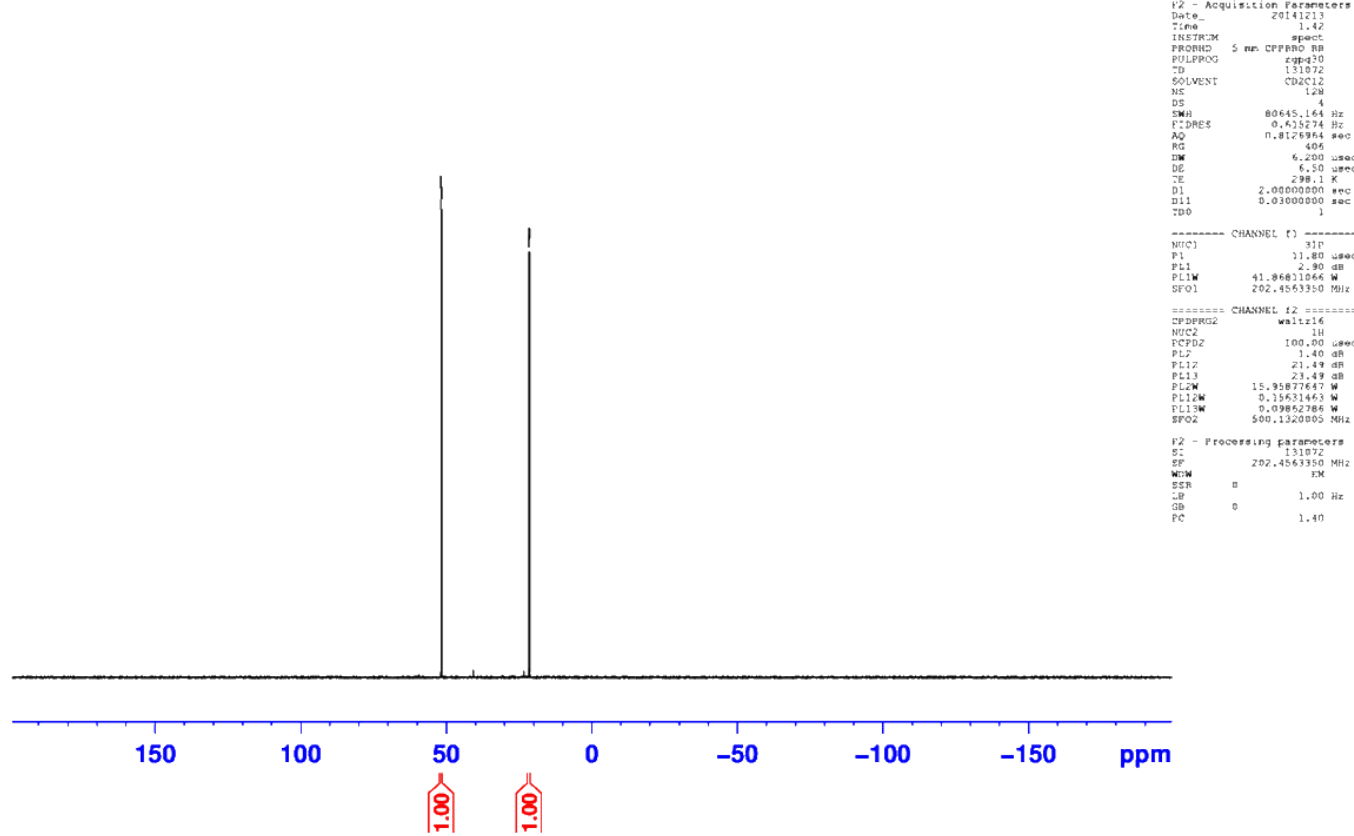

guest Molitor

SM504

APROTON16_PRODI CD2C12 \{E:\Bruker\Topspin\} User 13

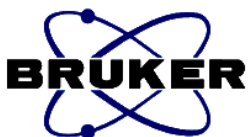

แू.

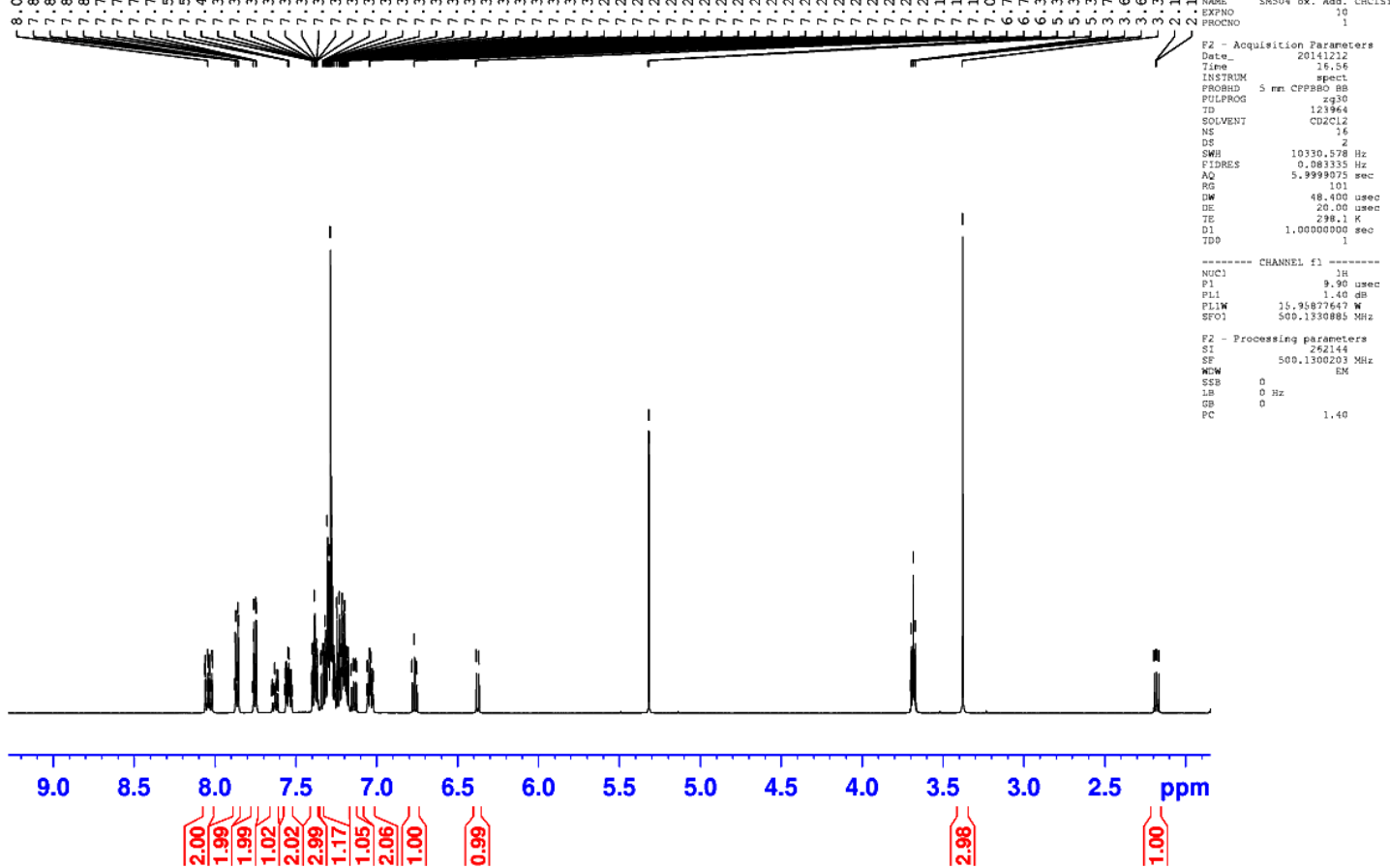

Figure S28. ${ }^{31} \mathrm{P}\left\{{ }^{1} \mathrm{H}\right\}$ NMR and ${ }^{1} \mathrm{H}$ NMR spectra of palladium complex $\mathbf{2 f}$. 
guest Molitor

SM504

AC13CPD_PRODI CD2C12 \{E: \Bruker $\backslash$ Topspin\} User 13

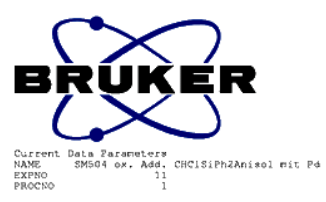

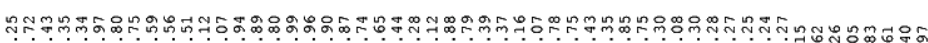

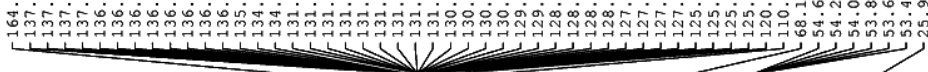

象?

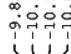

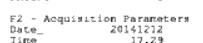
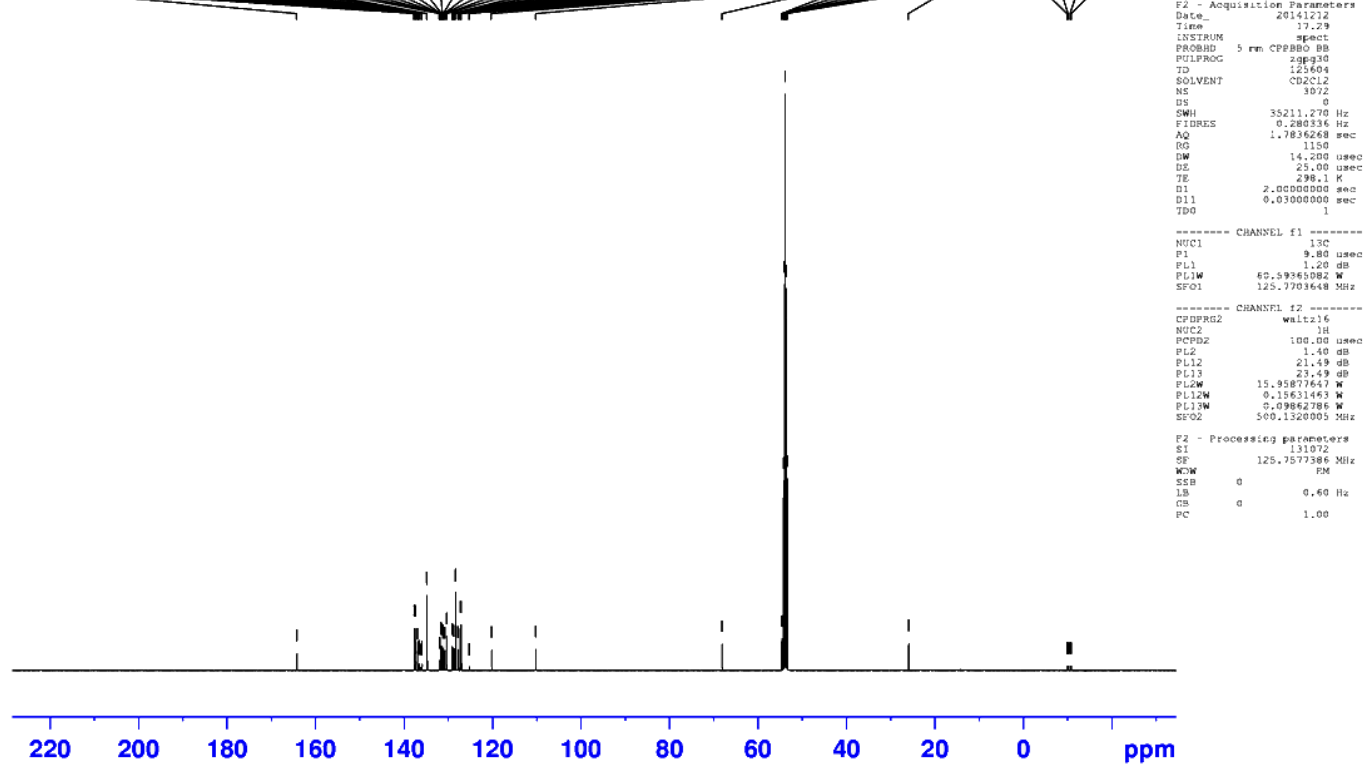

guest Molitor

SM50 4

AC13DEPT135_PRODI CD2C12 \{E:\Bruker\Topspin\} User 13
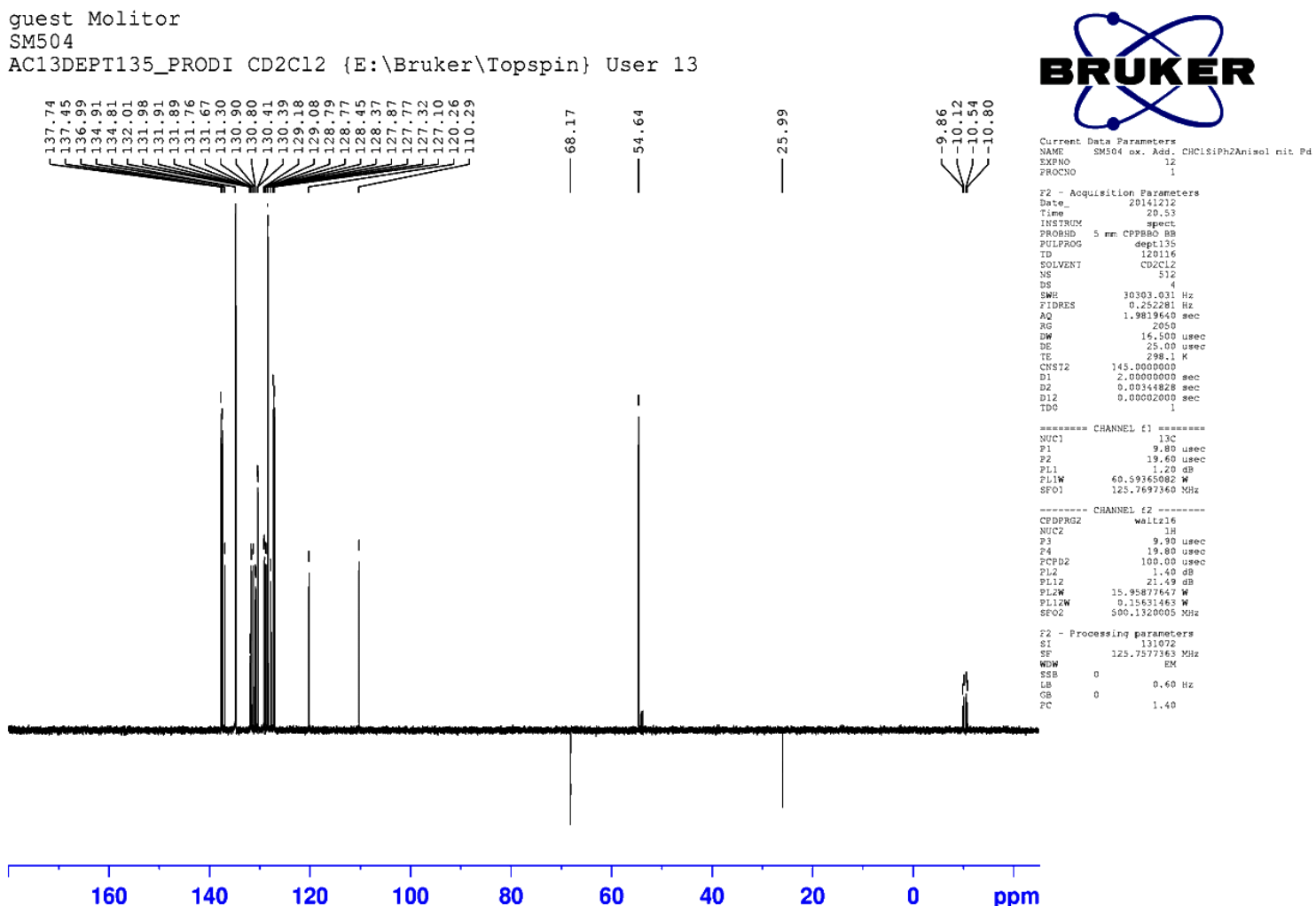

Figure S29. ${ }^{13} \mathrm{C}\left\{{ }^{1} \mathrm{H}\right\}$ NMR and ${ }^{13} \mathrm{C}\left\{{ }^{1} \mathrm{H}\right\}$-DEPT135 NMR spectra of palladium complex $\mathbf{2 f}$. 
guest Molitor

SM504

ASI29IG_PRODI CD2C12 \{E: \Bruker\Topspin\} User 13
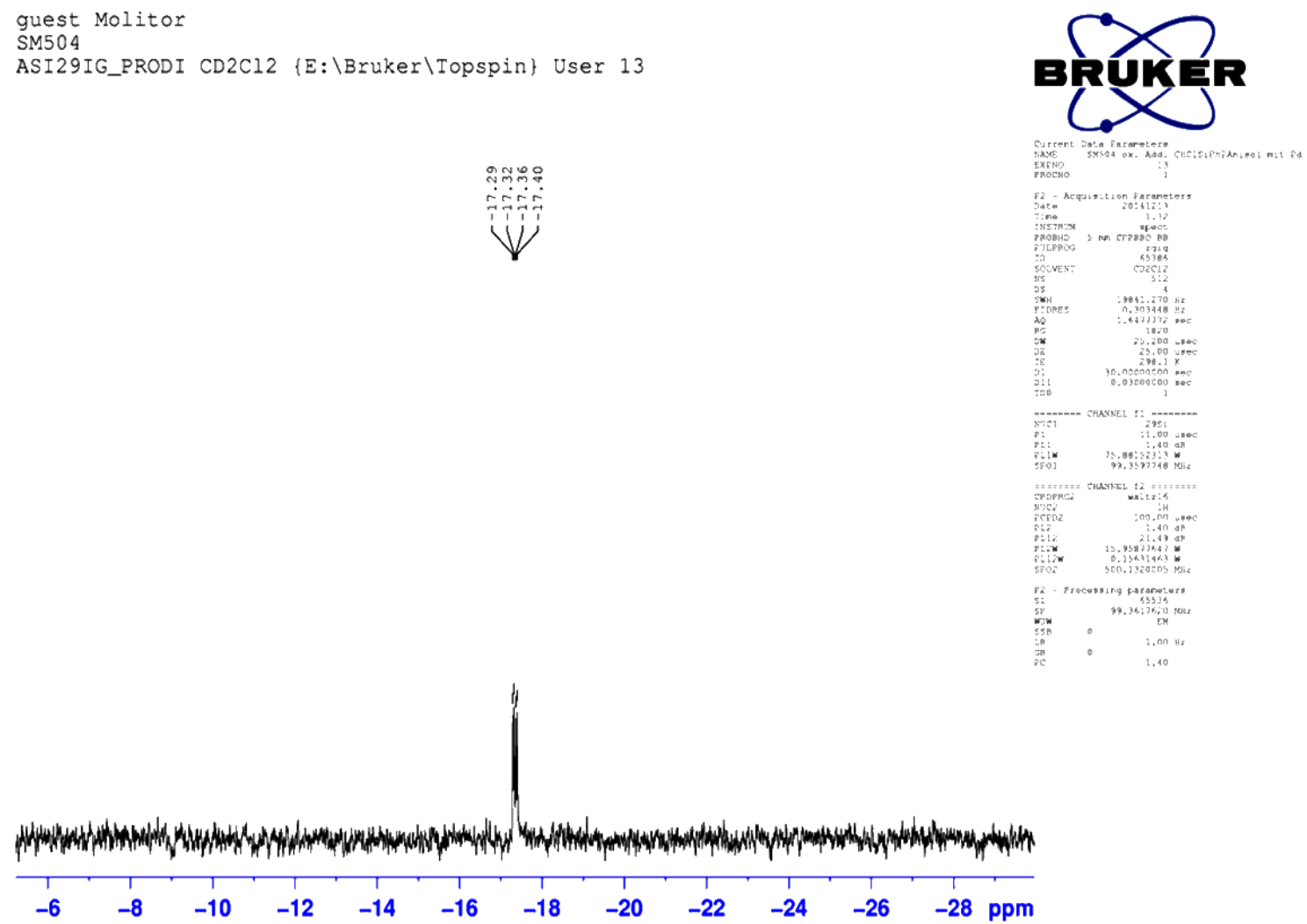

Figure S30. ${ }^{29} \mathrm{Si}\left\{{ }^{1} \mathrm{H}\right\}$ NMR spectrum of palladium complex $\mathbf{2 f}$. 
guest Molitor

SM4 65

AP31CPD CD2C12 \{E: \Bruker\Topspin\} User 50

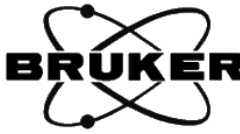

Current Data Parameters
WAME SM465 ortho Metallierung
14

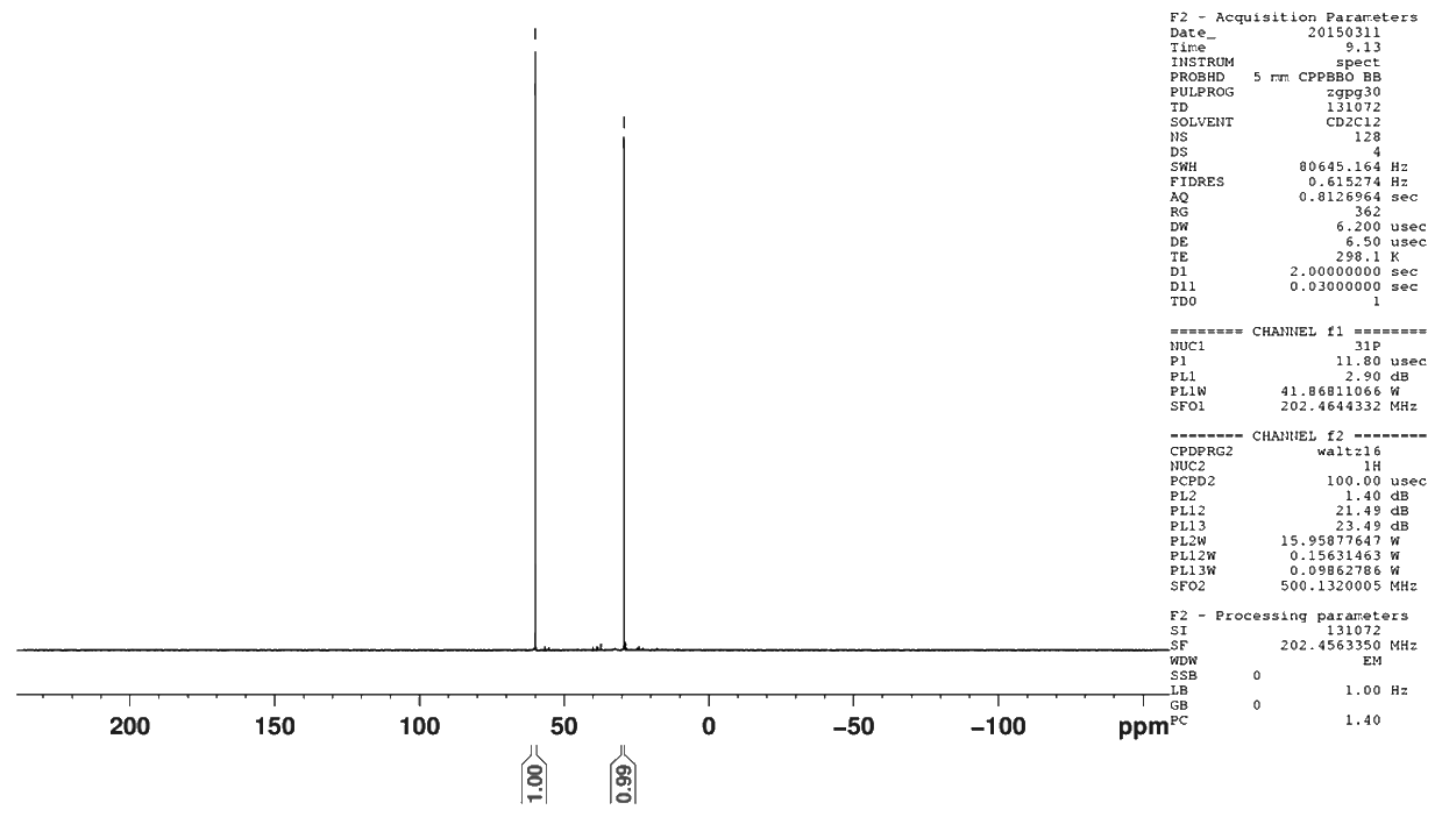

guest Molitor

APROTON16_PRODI CD2Cl2 \{E:\Bruker\Topspin\} User 50

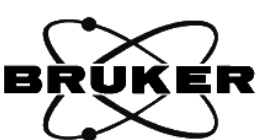

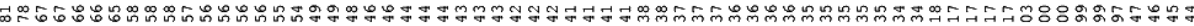
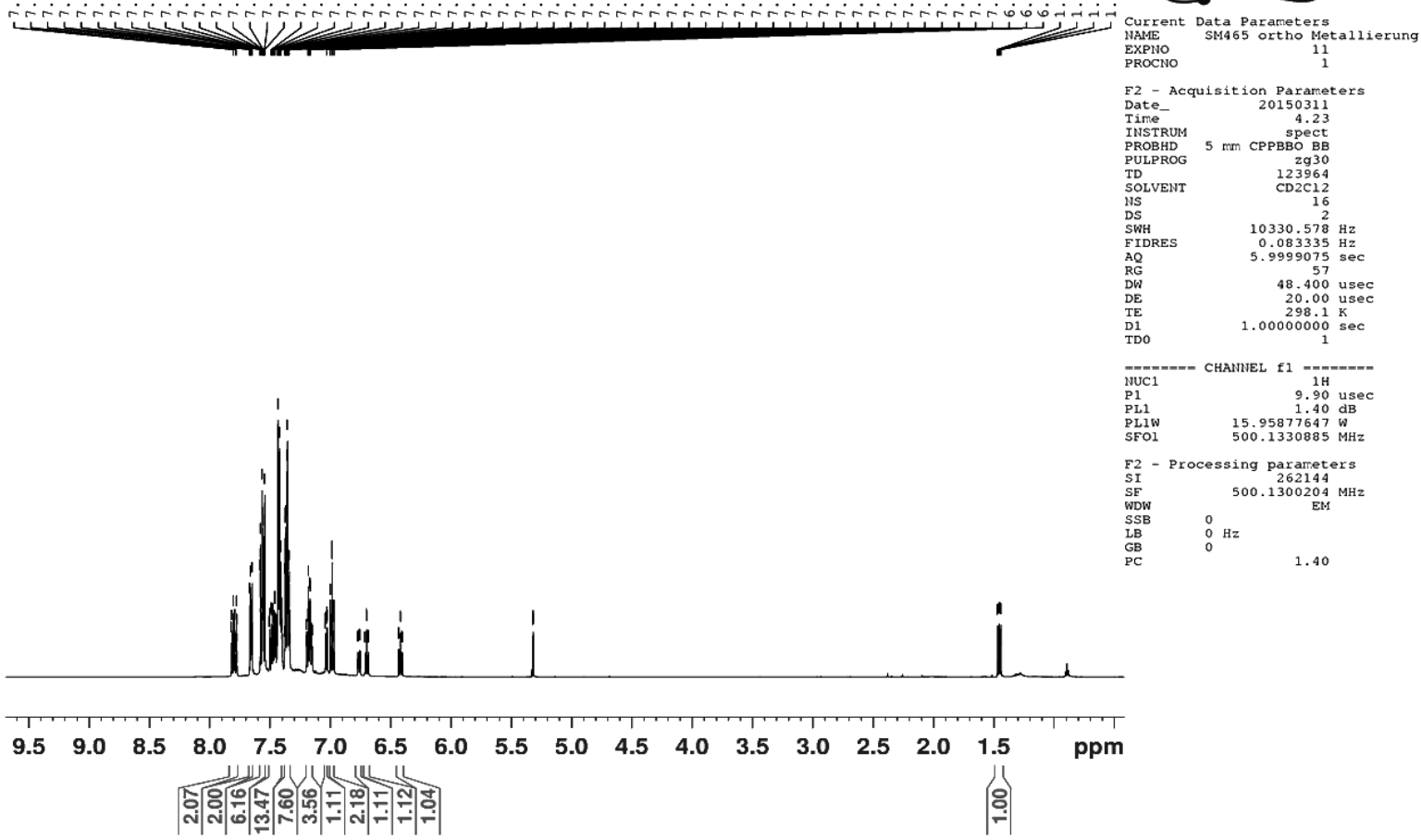

Figure S31. ${ }^{31} \mathrm{P}\left\{{ }^{1} \mathrm{H}\right\}$ NMR and ${ }^{1} \mathrm{H}$ NMR spectra of palladium complex 3. 

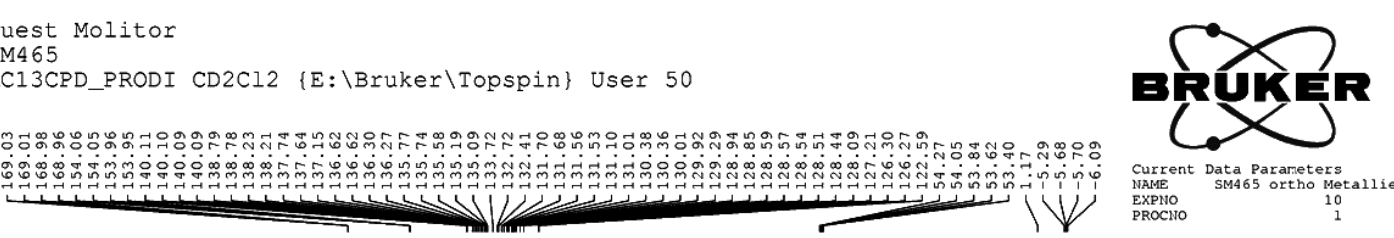

Current Data Parameters
NAME
EXPNO
SM465 ortho Metallierung
PROCNO

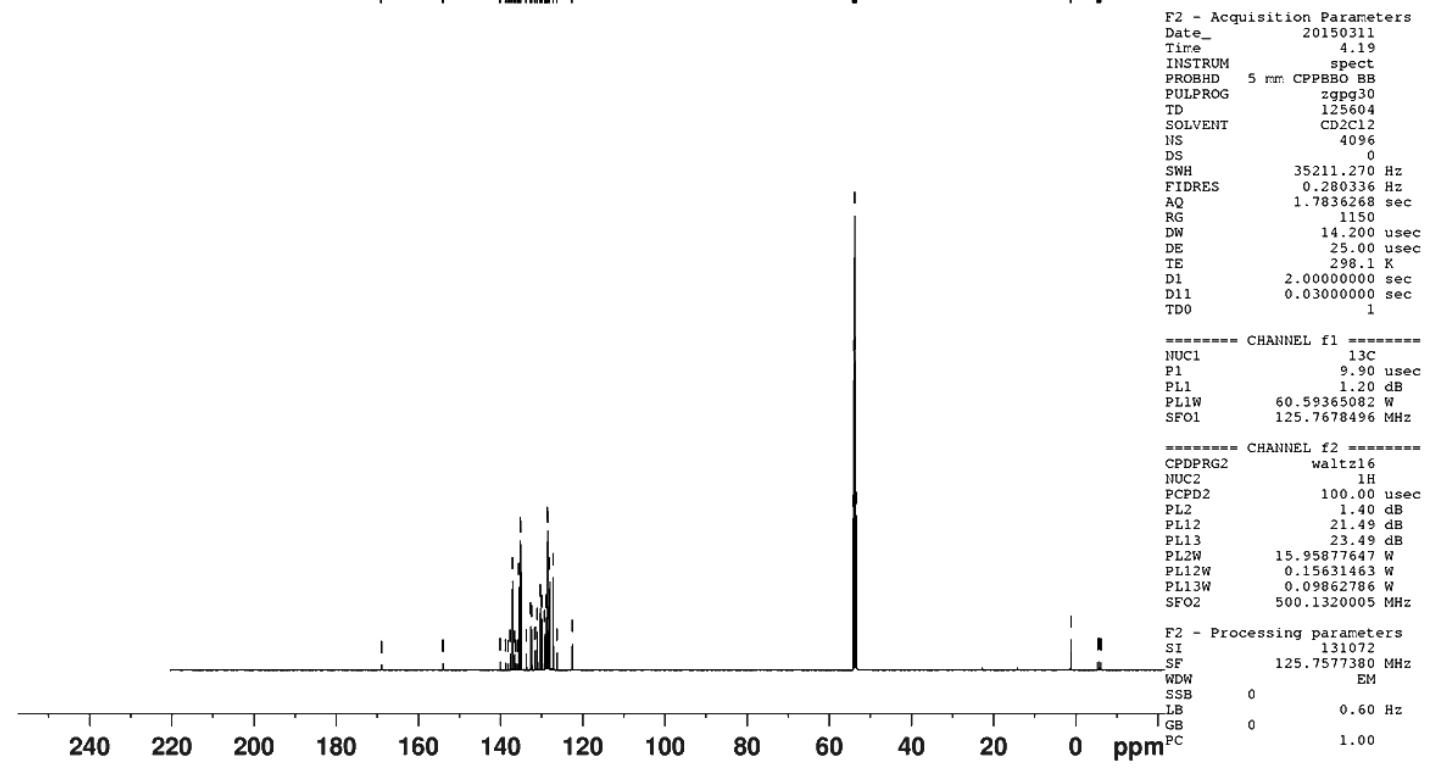

guest Molitor

SM4 65

AC13DEPT135_PRODI CD2C12 \{E:\Bruker\Topspin\} User 50
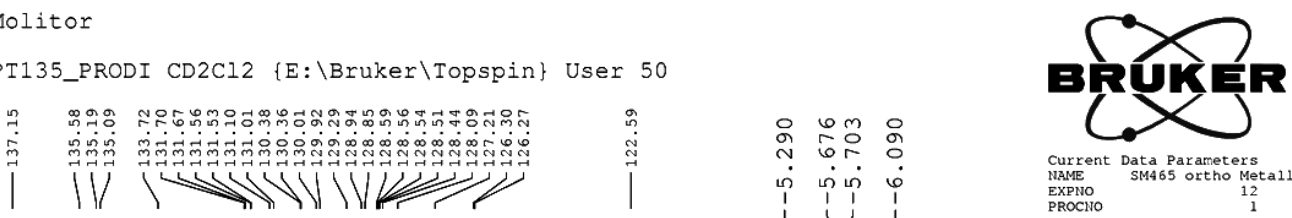
Current Data Parameters
NAME
EXPNO
SM465 ortho Metallierung
PROCNO
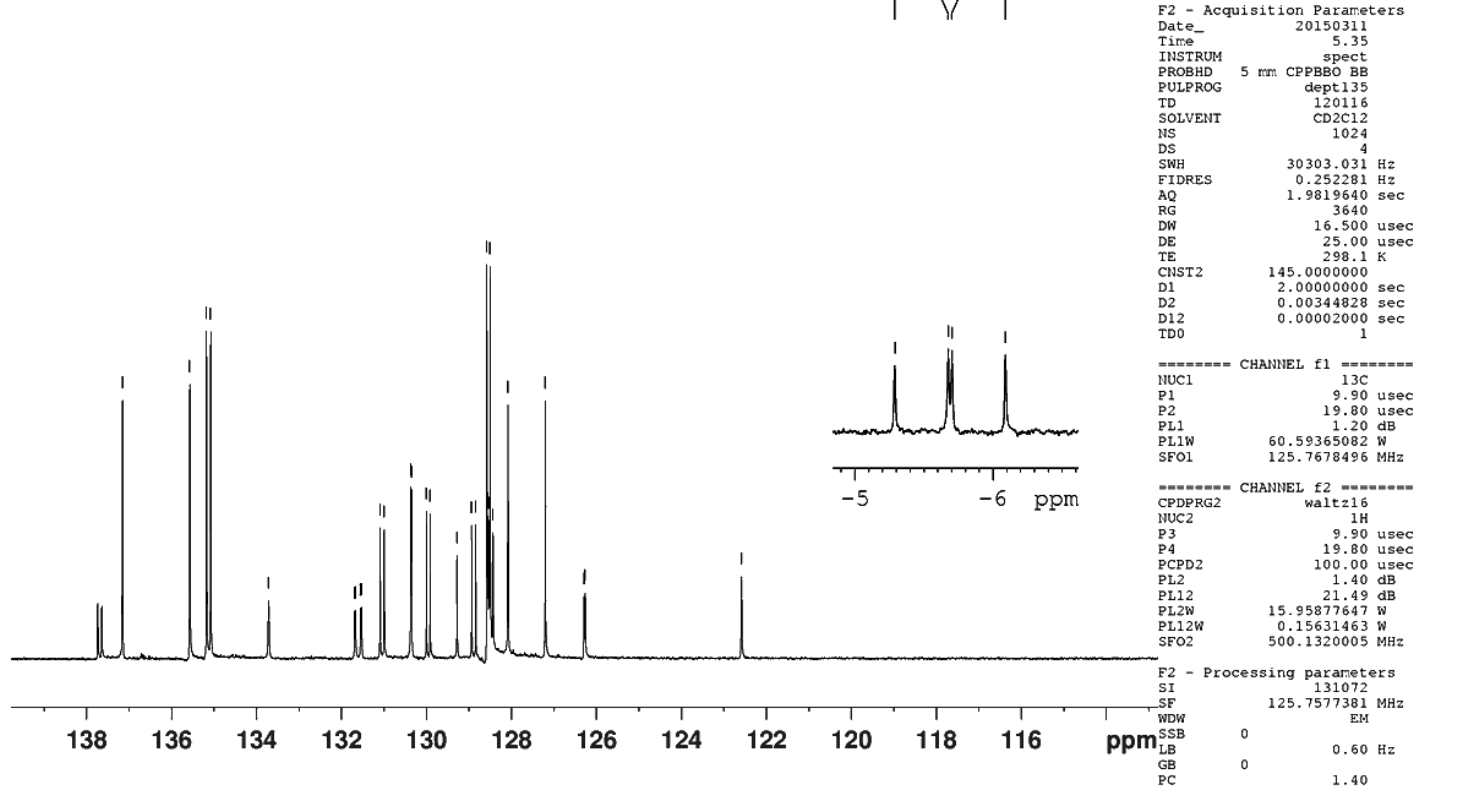

Figure S32. ${ }^{13} \mathrm{C}\left\{{ }^{1} \mathrm{H}\right\}$ NMR and ${ }^{13} \mathrm{C}\left\{{ }^{1} \mathrm{H}\right\}$-DEPT135 NMR spectra of palladium complex 3 . 
guest Molitor

SM465

ASI29IG_PRODI CD2C12 \{E: \Bruker\Topspin\} User 50

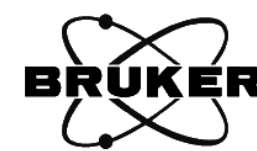

Current Data Parameters
NAME
SM465 ortho Metallierung

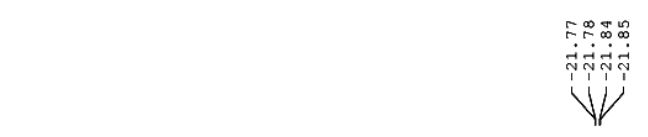

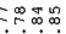

กิ

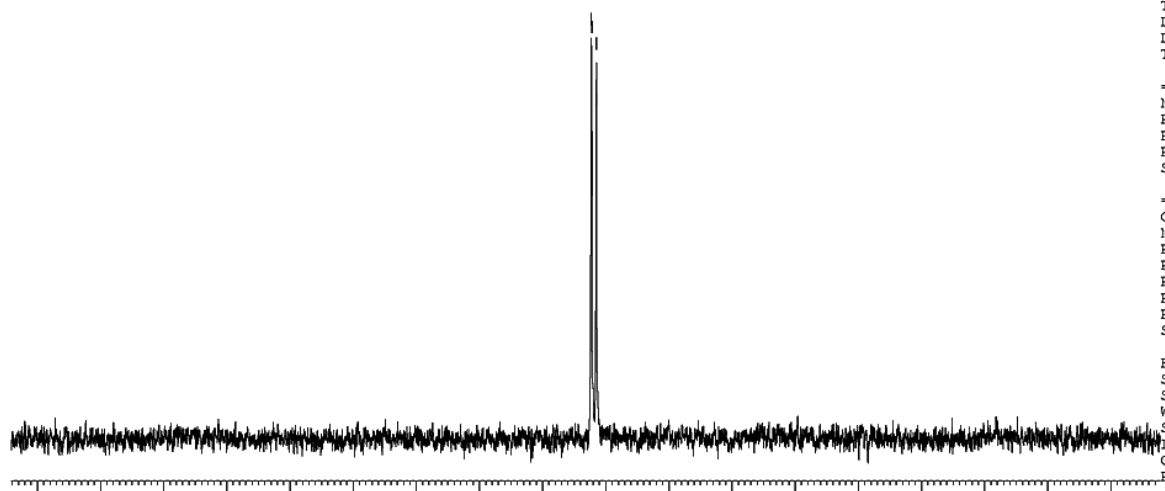

$\begin{array}{llllllllllllllllll}-13 & -14 & -15 & -16 & -17 & -18 & -19 & -20 & -21 & -22 & -23 & -24 & -25 & -26 & -27 & -28 & -29 & \text { ppm }\end{array}$
EXPNO
PROCNO

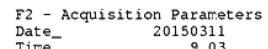

Date_ $\quad 20150311$

Time-

PULPROG $5 \mathrm{~mm}$ CPPBBO B

TD
SOLVENT
NS

SS

SWH

AQ

$R G$
$D W$
$D E$
$T E$
$T E$

D11
TD0

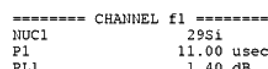

\begin{tabular}{l}
$75.88152313 \mathrm{~W}$ \\
PL1W \\
SFO1 \\
\hline
\end{tabular}

$======$
CPDPRG2

$\begin{array}{lr}\text { PCPD2 } & 100.00 \mathrm{usec} \\ \text { PL2 } & 1.40 \mathrm{~dB} \\ 21.49 \mathrm{~dB}\end{array}$

15.95877647 w
PL2W
0.15631463 W
F2 - Processing paraaneters
SI 5536
$\mathrm{SF}$
$\mathrm{WF}$

$0.30 \mathrm{~Hz}$

1.40

Figure S33. ${ }^{29} \mathrm{Si}\left\{{ }^{1} \mathrm{H}\right\}$ NMR spectrum of palladium complex $\mathbf{3}$. 
guest Molitor

M552d

AP31CPD C6D6 \{E: \Bruker \Topspin\} User 10

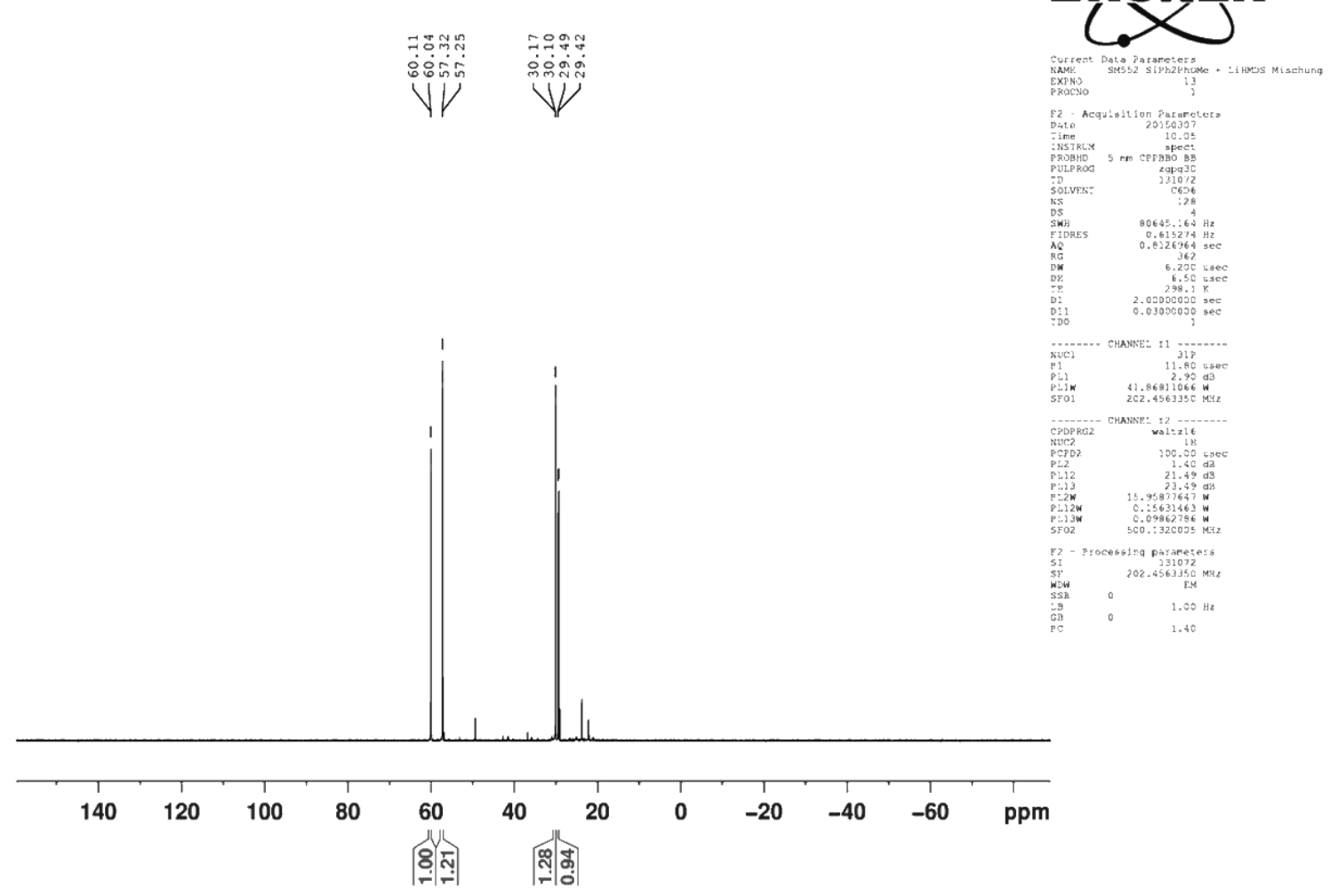

guest Molitor

SM552d

APROTON16_PRODI C6D6 \{E: \Bruker\Topspin\} User 10

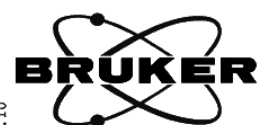

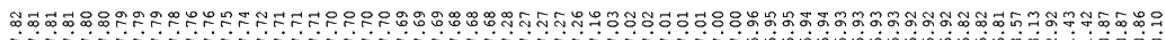
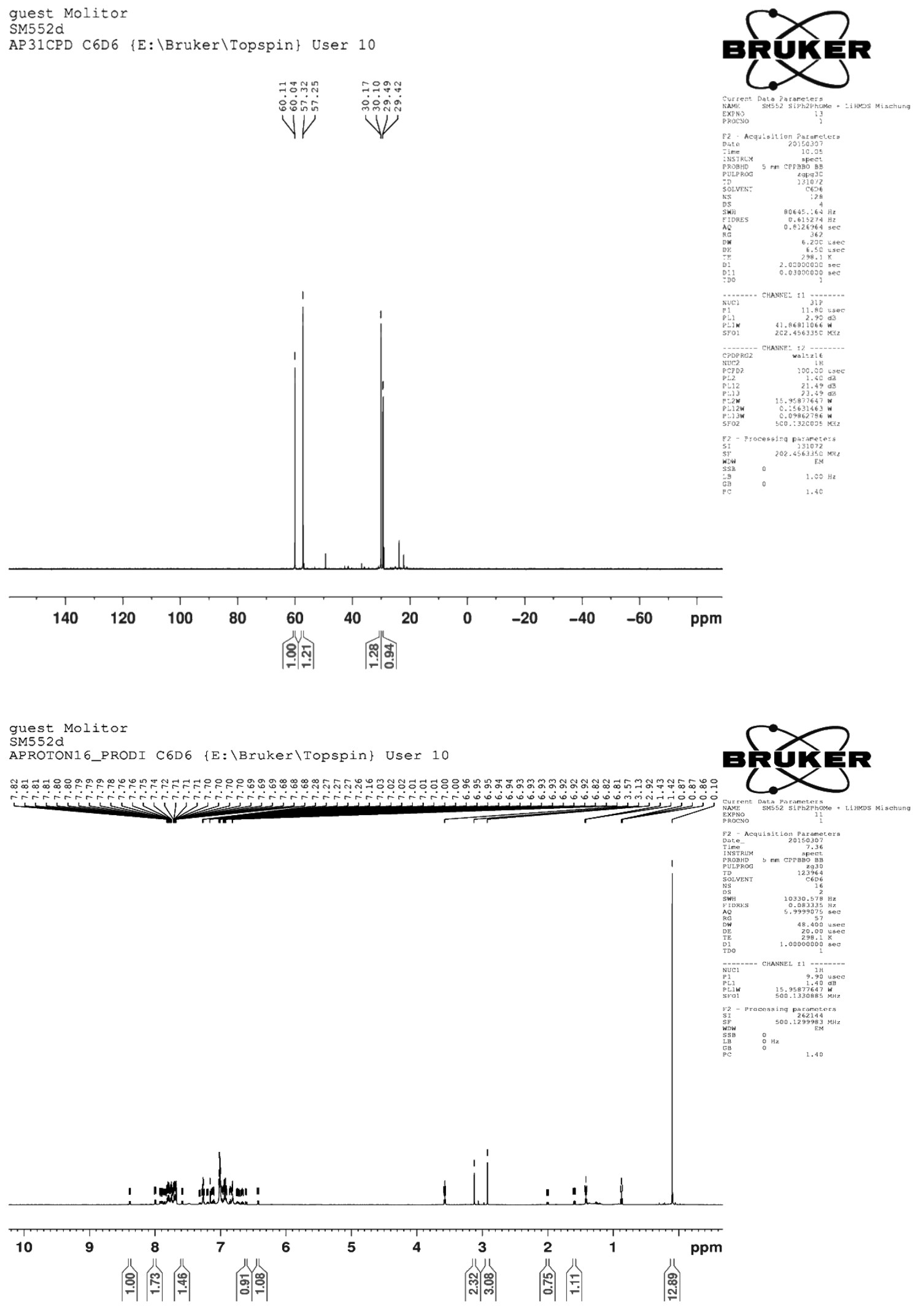

Figure S34. ${ }^{31} \mathrm{P}\left\{{ }^{1} \mathrm{H}\right\}$ NMR and ${ }^{1} \mathrm{H}$ NMR spectra of Isomers $\mathbf{4 a}$ and $\mathbf{4 b}$. 
AC13CPD_PRODI C6D 6 \{E: \Bruker $\backslash$ Topspin $\}$ User 10

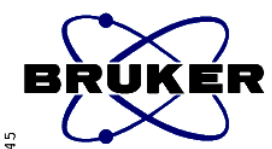

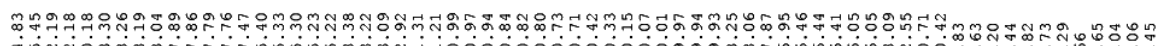

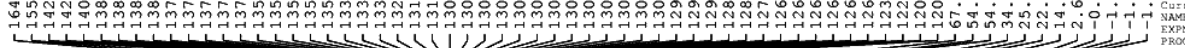

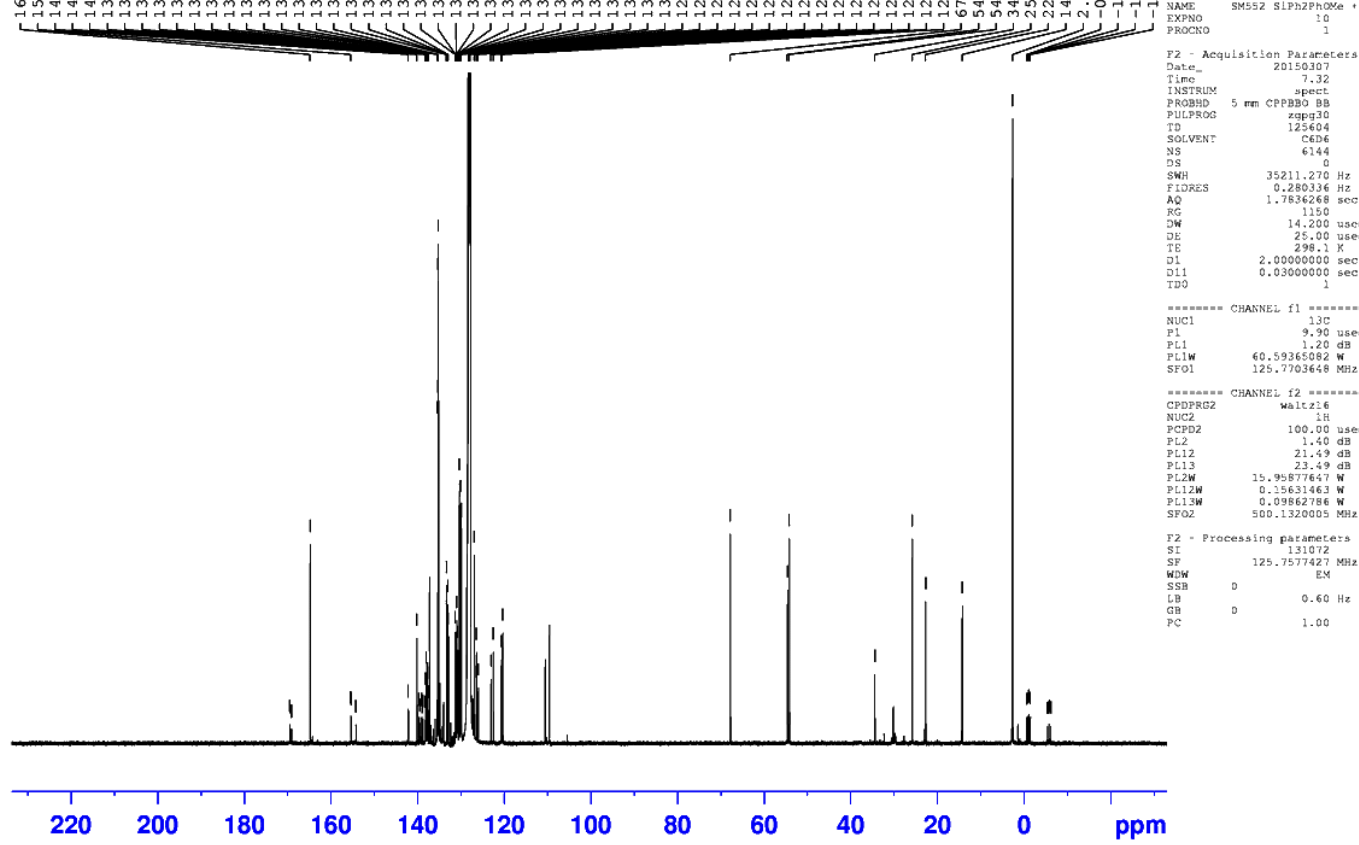

guest Molitor

SM552d

AC13DEPT135_PRODI C6D6 \{E:\Bruker\Topspin\} User 10

๓

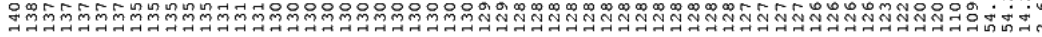
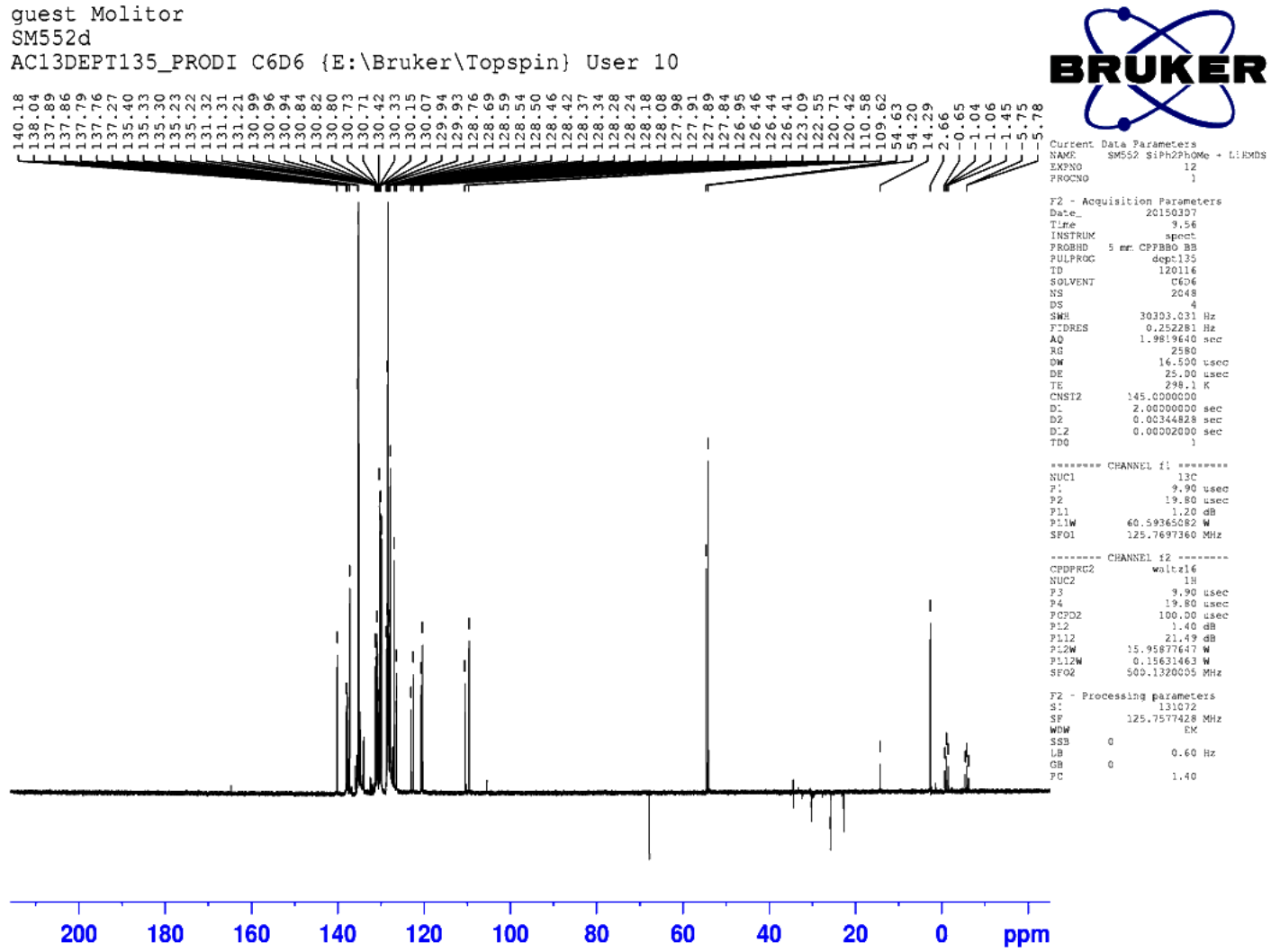

Figure S35. ${ }^{13} \mathrm{C}\left\{{ }^{1} \mathrm{H}\right\}$ NMR and ${ }^{13} \mathrm{C}\left\{{ }^{1} \mathrm{H}\right\}$-DEPT135 NMR spectra of Isomers $4 \mathbf{a}$ and $\mathbf{4 b}$. 
guest Molitor

SM552d

ASI29IG_PRODI C6D6 \{E:\Bruker\Topspin\} User 10
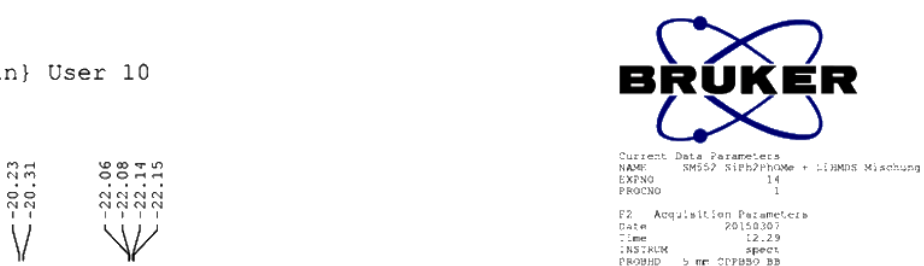

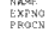
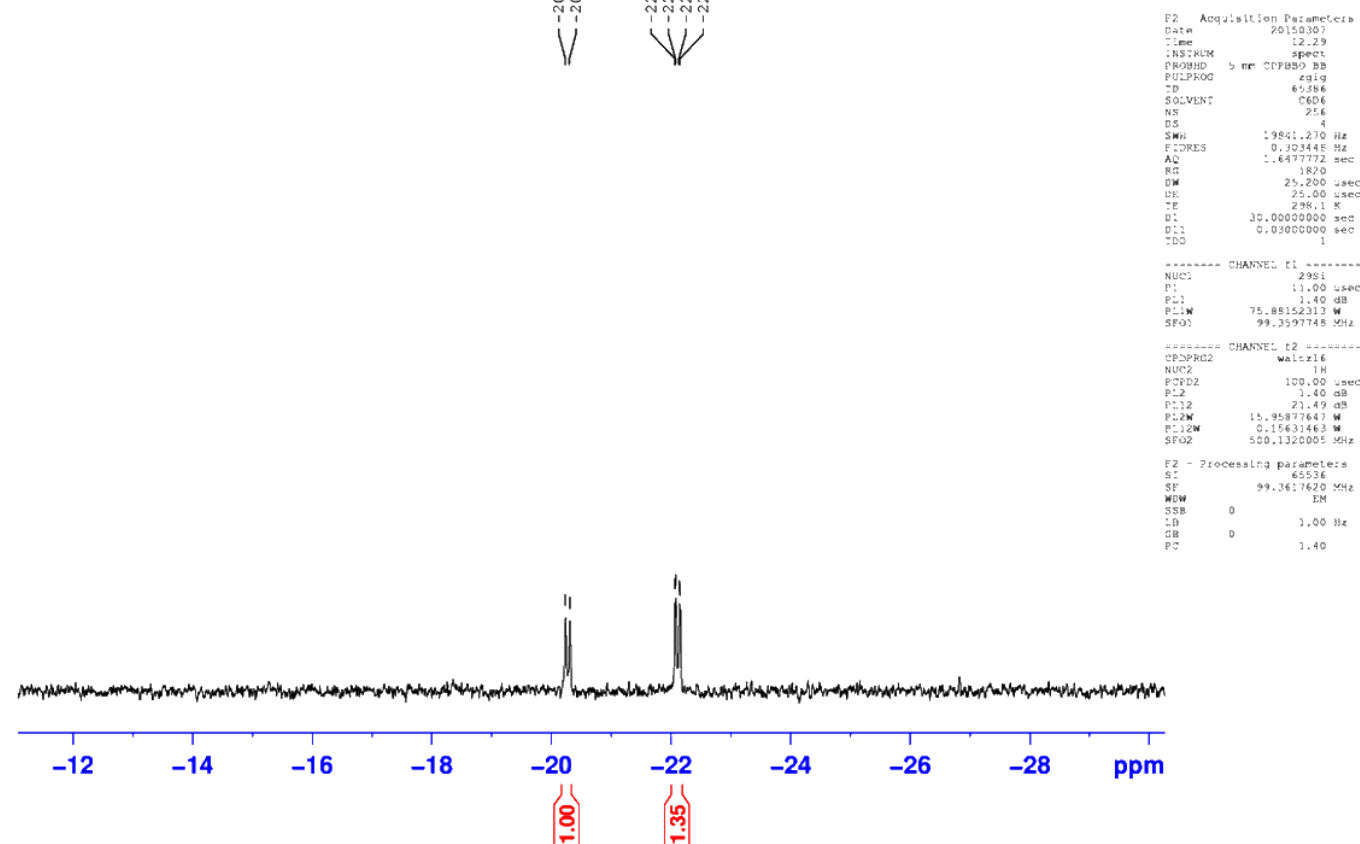

Figure S36. ${ }^{29} \mathrm{Si}\left\{{ }^{1} \mathrm{H}\right\}$ NMR spectrum spectra of Isomers $\mathbf{4 a}$ and $\mathbf{4 b}$. 
guest Molitor

SM $537 \mathrm{C}$

AP31CPD C6D 6 \{E: \Bruker \Topspin\} User 13

ปั่

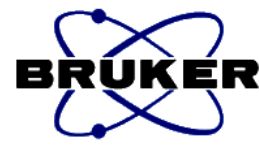

Current Data Parameters
NAME SM537 Cyclobutan SiMe 3

EXPNO
PROCNO
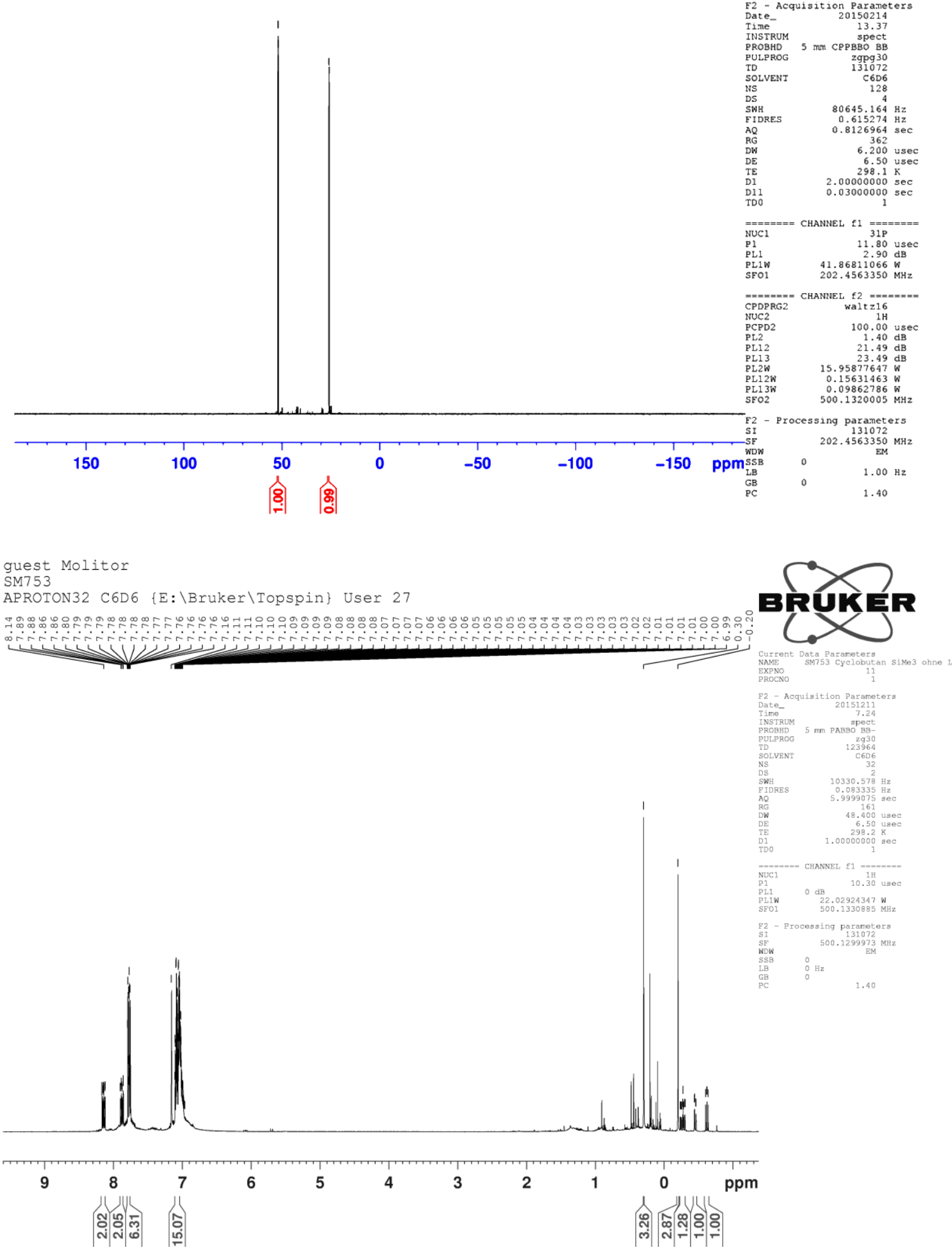

Figure S37. ${ }^{31} \mathrm{P}\left\{{ }^{1} \mathrm{H}\right\}$ NMR and ${ }^{1} \mathrm{H}$ NMR spectra of palladium complex 5. 


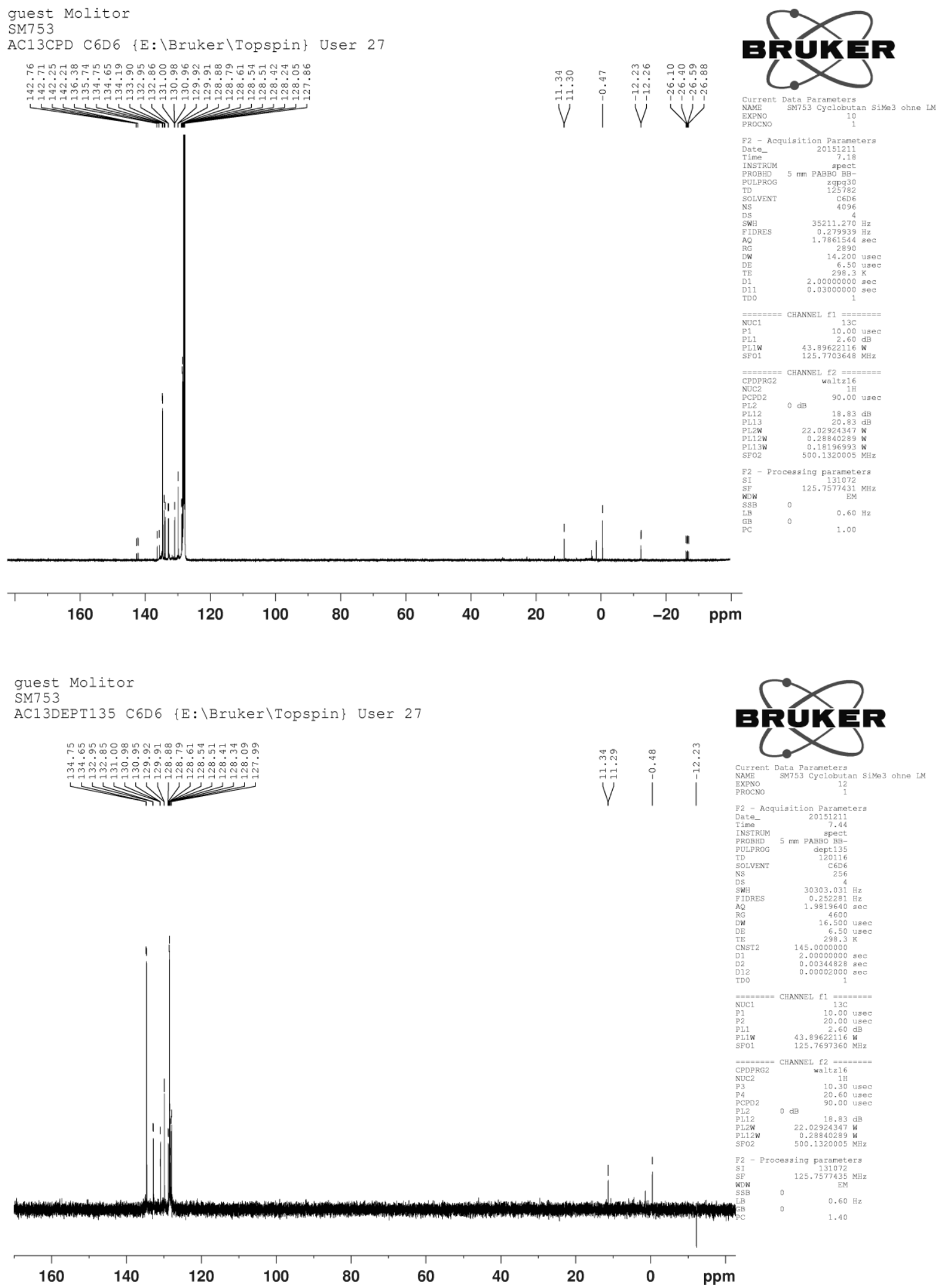

Figure S38. ${ }^{13} \mathrm{C}\left\{{ }^{1} \mathrm{H}\right\}$ NMR and ${ }^{13} \mathrm{C}\left\{{ }^{1} \mathrm{H}\right\}$-DEPT135 NMR spectra of palladium complex 5. 
guest Molitor

SM $537 \mathrm{C}$

ASI29IG_PRODI C6D6 \{E:\Bruker\Topspin\} User 13
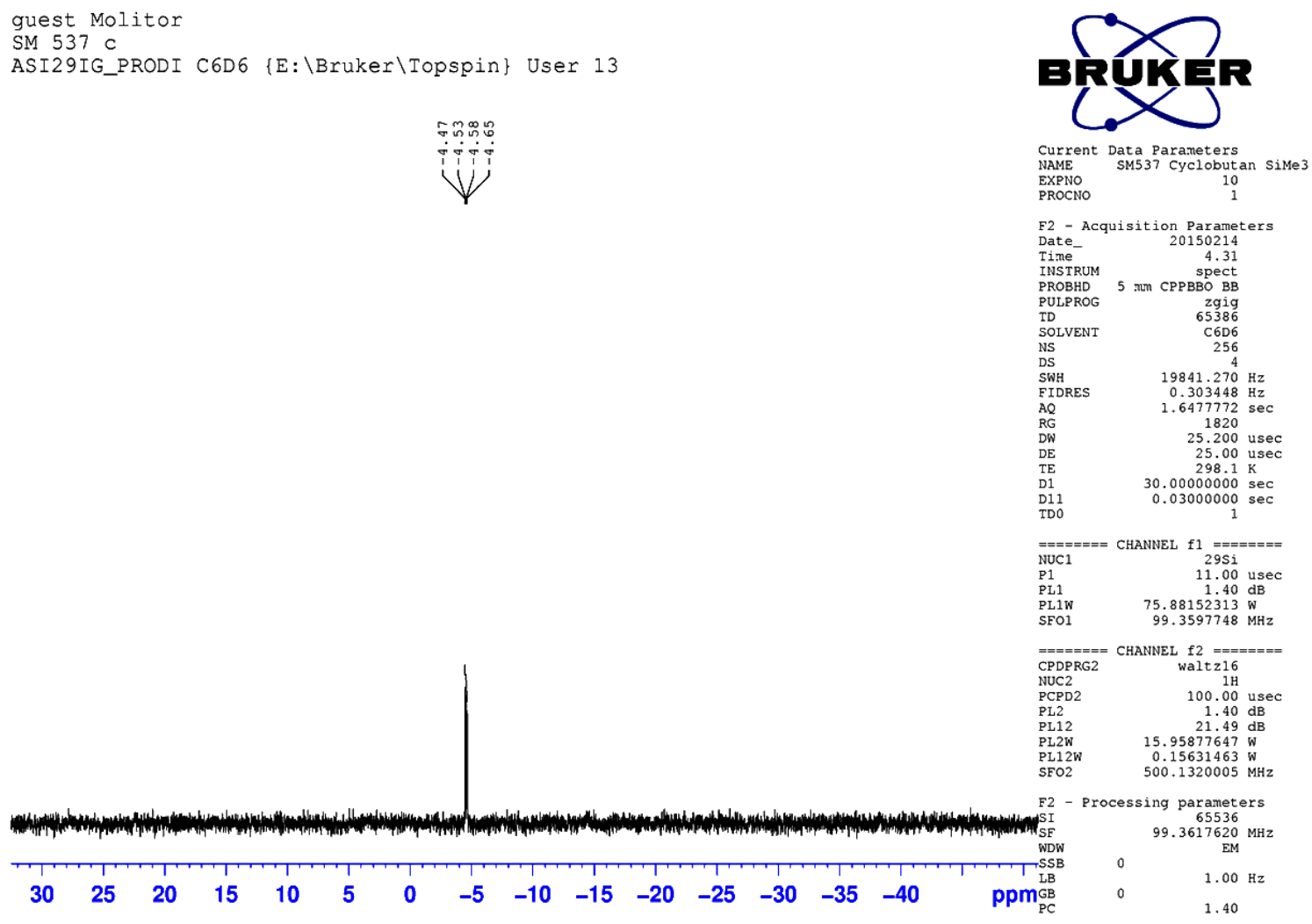

Figure S39. ${ }^{29} \mathrm{Si}\left\{{ }^{1} \mathrm{H}\right\}$ NMR spectrum of palladium complex $\mathbf{5}$. 
guest Molitor

SM5 46

AP31CPD C6D6 \{E: \Bruker \Topspin\} User 49

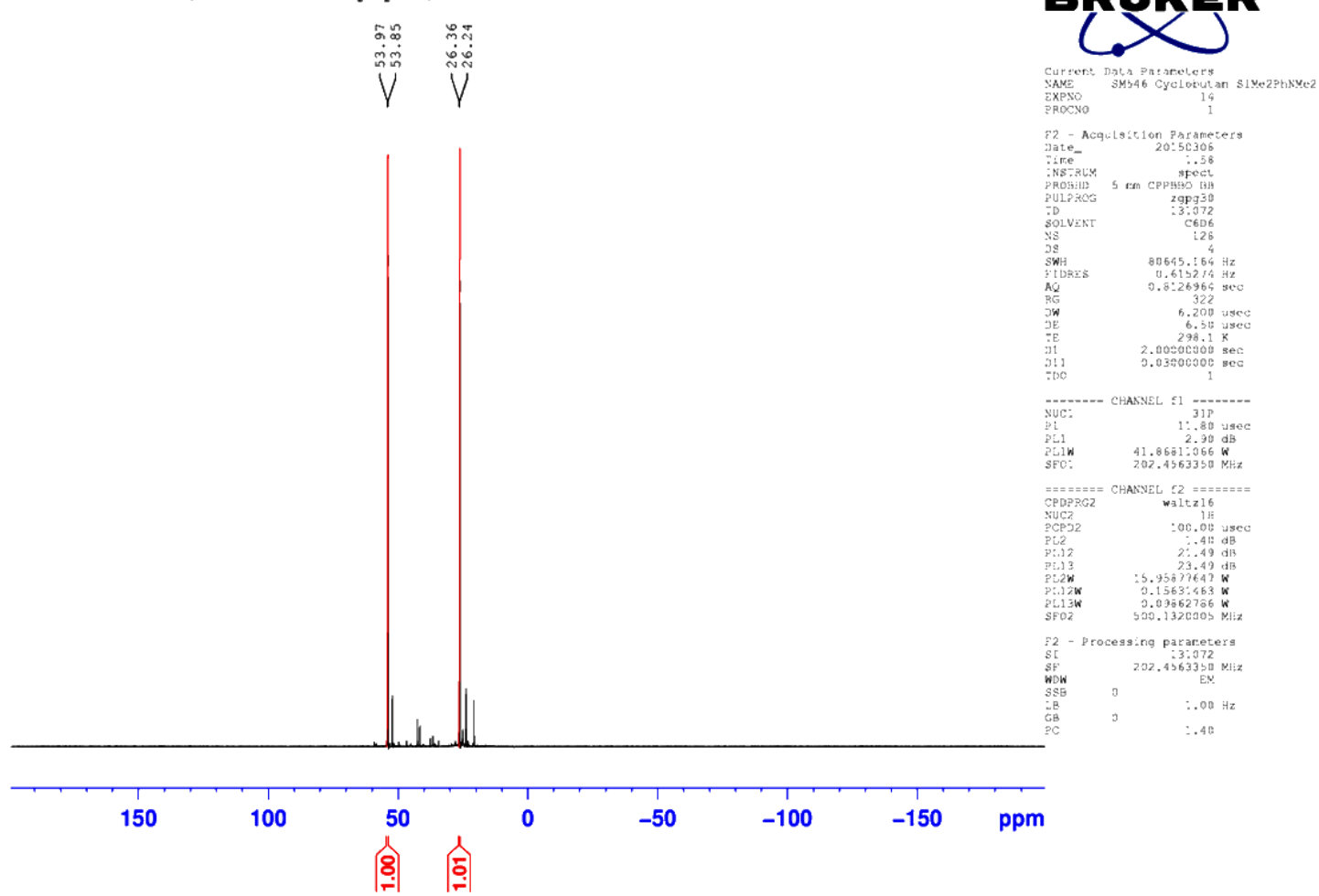

guest Molitor

SM5 46

APROTON16_PRODI C6D6 \{E:\Bruker\Topspin\} User 49

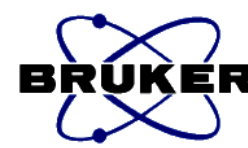

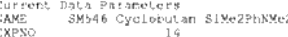

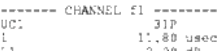

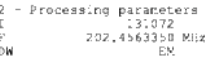
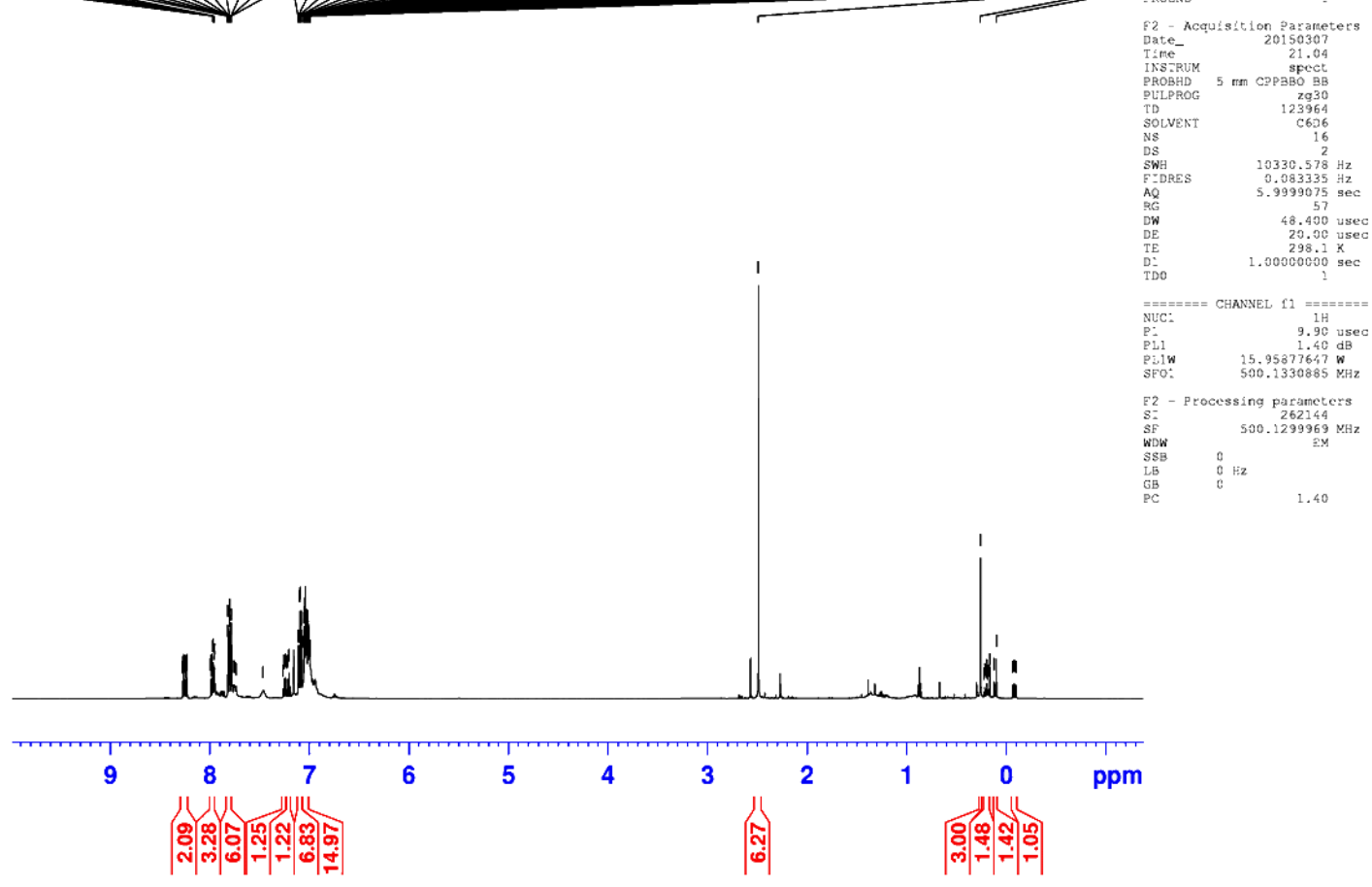

Figure S40. ${ }^{31} \mathrm{P}\left\{{ }^{1} \mathrm{H}\right\}$ NMR and ${ }^{1} \mathrm{H}$ NMR spectra of palladium complex 6. 


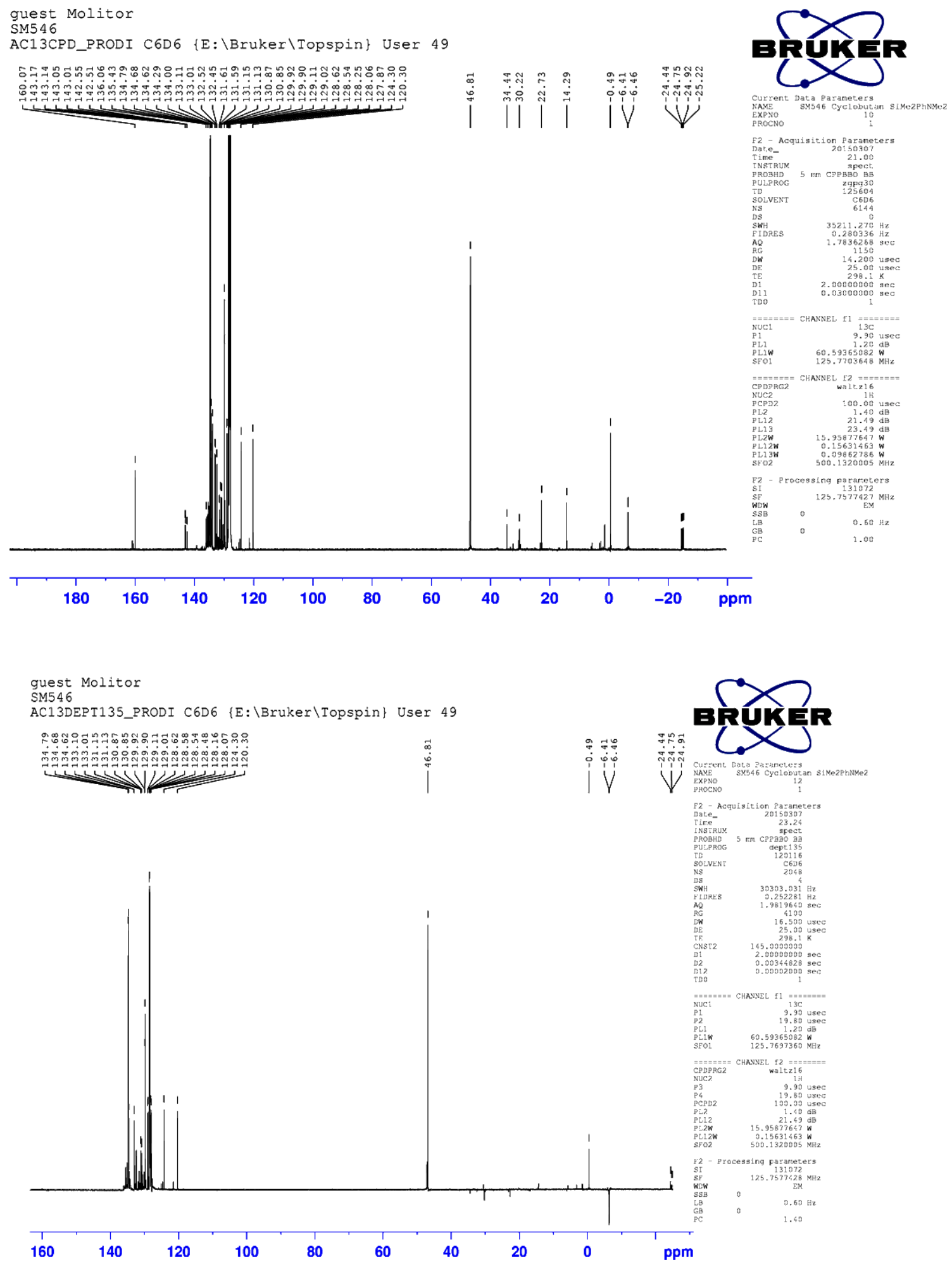

Figure S41. ${ }^{13} \mathrm{C}\left\{{ }^{1} \mathrm{H}\right\}$ NMR and ${ }^{13} \mathrm{C}\left\{{ }^{1} \mathrm{H}\right\}$-DEPT135 NMR spectra of palladium complex 6. 


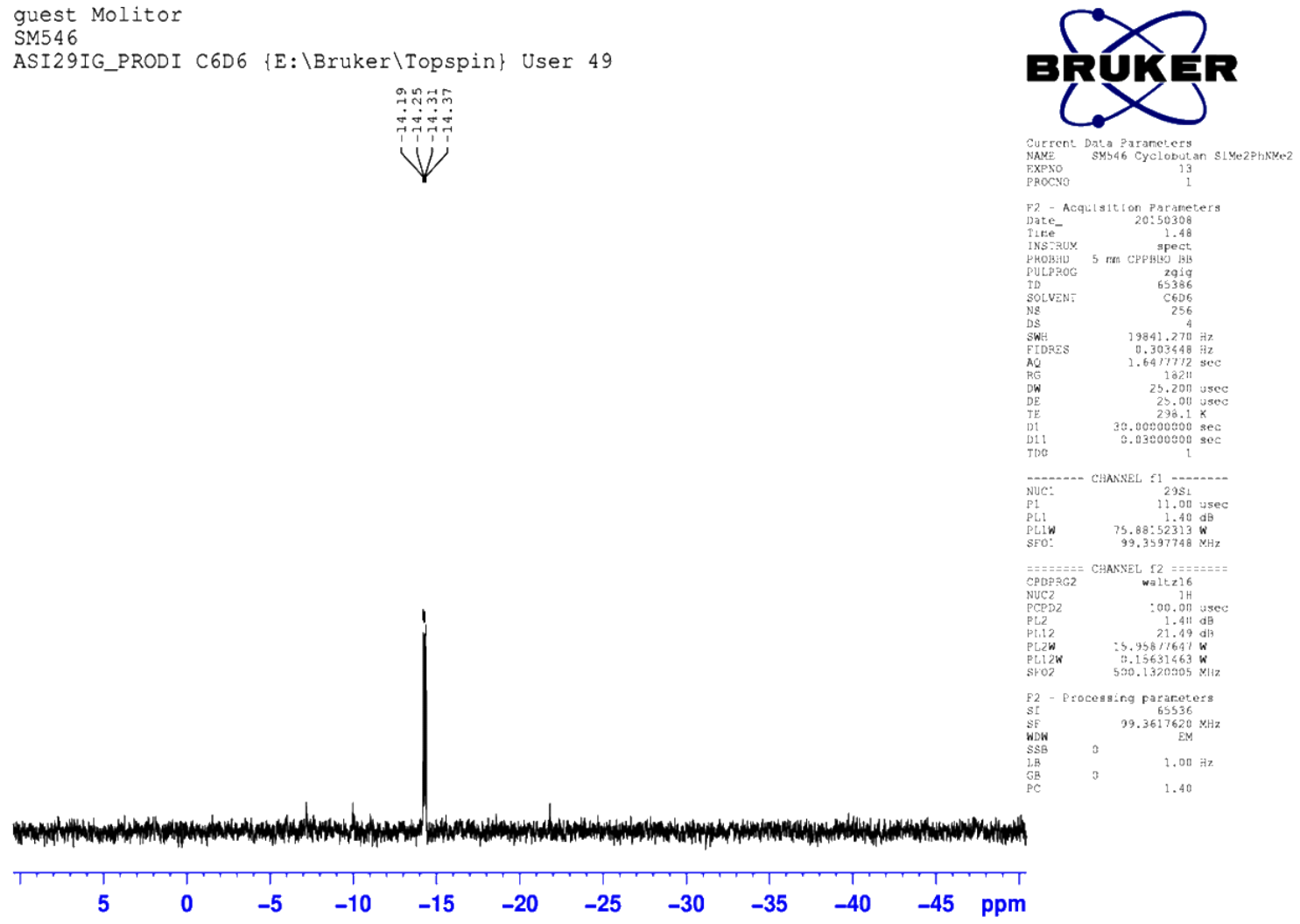

Figure $\mathbf{S 4 2} .{ }^{29} \mathrm{Si}\left\{{ }^{1} \mathrm{H}\right\}$ NMR spectrum of palladium complex 6 . 


\section{Crystal Structure Determination}

Data collection of all compounds was conducted with a Bruker APEX2-CCD (D8 three-circle goniometer). The structures were solved using direct methods, refined with the Shelx software package $^{[2]}$ and expanded using Fourier techniques. The crystals of all compounds were mounted in an inert oil (perfluoropolyalkylether). Crystal structure determinations were effected at $100 \mathrm{~K}$. Crystallographic data (excluding structure factors) have been deposited with the Cambridge Crystallographic Data Centre as supplementary publication no. CCDC-1432200-1432212. Copies of the data can be obtained free of charge on application to Cambridge Crystallographic Data Centre, 12 Union Road, Cambridge CB2 1EZ, UK; [fax: (+44) 1223-336-033; email: deposit@ @cdc.cam.ac.uk].

Table S1a. Data collection and structure refinement details for compounds $\mathbf{1 a}, \mathbf{1 b}$ and $\mathbf{1 d}$.

\begin{tabular}{|c|c|c|c|}
\hline Compound & $1 \mathbf{a}$ & $\mathbf{1 b}$ & 1d \\
\hline CCDC No. & CCDC 1432200 & CCDC 1432201 & CCDC 1432202 \\
\hline Formula & $\mathrm{C}_{31} \mathrm{H}_{26} \mathrm{ClPSSi}$ & $\mathrm{C}_{31} \mathrm{H}_{26}$ IPSSi & $\mathrm{C}_{16} \mathrm{H}_{20} \mathrm{IPSSi}$ \\
\hline $\begin{array}{l}\text { Formula weight } \\
{\left[\mathrm{g} \cdot \mathrm{mol}^{-1}\right]}\end{array}$ & 525.09 & 616.54 & 430.34 \\
\hline Temperature [K] & $100(2)$ & $100(2)$ & $100(2)$ \\
\hline Wave length [̊̊] & 0.71073 & 0.71073 & 0.71073 \\
\hline Crystal system & monoclinic & monoclinic & monoclinic \\
\hline Space group & $P 2_{1} / \mathrm{n}(12)$ & $P 2_{1} / \mathrm{n}(12)$ & $P 2_{1} / \mathrm{c}(12)$ \\
\hline $\mathrm{a}[\AA]$ & $9.6136(8)$ & $10.0668(5)$ & $8.6586(4)$ \\
\hline $\mathrm{b}[\AA]$ & $23.5656(19)$ & $23.4116(12)$ & $12.4576(5)$ \\
\hline$c[\AA]$ & $12.2521(10)$ & $12.4898(8)$ & $16.9640(7)$ \\
\hline$\alpha\left[^{\circ}\right]$ & 90 & 90 & 90 \\
\hline$\beta\left[^{\circ}\right]$ & $110.219(3)$ & $112.127(2)$ & $103.642(1)$ \\
\hline$\gamma\left[{ }^{\circ}\right]$ & 90 & 90 & 90 \\
\hline Volume $\left[\AA^{3}\right]$ & $2604.7(4)$ & $2726.8(3)$ & $1778.21(13)$ \\
\hline $\mathrm{Z}$ & 4 & 4 & 4 \\
\hline Calc. density $\left[\mathrm{Mg} \cdot \mathrm{m}^{-3}\right]$ & 1.339 & 1.502 & 1.607 \\
\hline$\mu\left(\mathrm{Mo}_{\mathrm{K} \alpha}\right)\left[\mathrm{mm}^{-1}\right]$ & 0.354 & 1.372 & 2.065 \\
\hline $\mathrm{F}(000)$ & 1096 & 1240 & 856 \\
\hline $\begin{array}{l}\text { Crystal dimensions } \\
{[\mathrm{mm}]}\end{array}$ & $0.26 \times 0.21 \times 0.15$ & $0.40 \times 0.30 \times 0.18$ & $0.32 \times 0.23 \times 0.07$ \\
\hline Theta range $\left[{ }^{\circ}\right]$ & 1.73 to 26.41 & 1.96 to 25.00 & 2.47 to 25.00 \\
\hline \multirow[t]{3}{*}{ Index ranges } & $-12 \leq \mathrm{h} \leq 12$ & $-11 \leq \mathrm{h} \leq 11$ & $-10 \leq h \leq 10$ \\
\hline & $-29 \leq \mathrm{k} \leq 29$ & $-27 \leq \mathrm{k} \leq 24$ & $-14 \leq \mathrm{k} \leq 14$ \\
\hline & $-15 \leq 1 \leq 15$ & $-14 \leq 1 \leq 14$ & $-20 \leq 1 \leq 20$ \\
\hline Reflections collected & 41234 & 19430 & 15427 \\
\hline Independent reflections & $5335[$ Rint $=0.0399]$ & $4799[$ Rint $=0.0252]$ & $3119[$ Rint $=0.0345]$ \\
\hline $\begin{array}{l}\text { Data/Restraints/Paramet } \\
\text { er }\end{array}$ & $5335 / 0 / 316$ & 4799 / 0 / 316 & $3119 / 0 / 184$ \\
\hline Goodness-of-fit on $\mathrm{F}^{2}$ & 1.014 & 1.067 & 1.028 \\
\hline $\begin{array}{l}\text { Final } R \text { indices } \\
{[\mathrm{I}>2 \operatorname{sigma}(\mathrm{I})]}\end{array}$ & $\begin{array}{l}R 1=0.0339 \\
\mathrm{w} R 2=0.0863\end{array}$ & $\begin{array}{l}R 1=0.0207 \\
\mathrm{w} R 2=0.0430\end{array}$ & $\begin{array}{l}R 1=0.0217 \\
\mathrm{w} R 2=0.0459\end{array}$ \\
\hline$R$ indices (all data) & $\begin{array}{l}\mathrm{R} 1=0.0432 \\
\mathrm{wR} 2=0.0911\end{array}$ & $\begin{array}{l}\mathrm{R} 1=0.0247 \\
\mathrm{wR} 2=0.0443\end{array}$ & $\begin{array}{l}\mathrm{R} 1=0.0276 \\
\mathrm{wR} 2=0.0486\end{array}$ \\
\hline $\begin{array}{l}\text { Largest diff. peak and } \\
\text { hole hole }\left[\mathrm{e} \cdot \AA^{-3}\right]\end{array}$ & 0.463 and -0.502 & 0.401 and -0.402 & 0.458 and -0.349 \\
\hline
\end{tabular}


Table S1b. Data collection and structure refinement details for compounds 1e and $\mathbf{1 f}$.

\begin{tabular}{|c|c|c|}
\hline Compound & $1 \mathrm{e}$ & 1f \\
\hline CCDC No. & CCDC 1432203 & CCDC 1432204 \\
\hline Formula & $\mathrm{C}_{23} \mathrm{H}_{27} \mathrm{ClNPSSi}$ & $\mathrm{C}_{32} \mathrm{H}_{28} \mathrm{ClOPSSi}$ \\
\hline $\begin{array}{l}\text { Formula weight } \\
{\left[\mathrm{g} \cdot \mathrm{mol}^{-1}\right]}\end{array}$ & 444.03 & 555.11 \\
\hline Temperature $[\mathrm{K}]$ & $100(2)$ & $100(2)$ \\
\hline Wave length $[\AA]$ & 0.71073 & 0.71073 \\
\hline Crystal system & orthorhombic & triclinic \\
\hline Space group & $P 2_{1} 2_{1} 2_{1}(19)$ & $P-1(2)$ \\
\hline $\mathrm{a}[\AA]$ & $8.7025(4)$ & $10.9149(7)$ \\
\hline $\mathrm{b}[\AA ̊ \cap]$ & $11.0106(5)$ & $11.5973(7)$ \\
\hline c $[\AA]$ & 24.1632(12) & $11.6182(7)$ \\
\hline$\alpha\left[^{\circ}\right]$ & 90 & $107.644(2)$ \\
\hline$\beta\left[{ }^{\circ}\right]$ & 90 & $94.044(2)$ \\
\hline$\gamma\left[{ }^{\circ}\right]$ & 90 & $95.380(2)$ \\
\hline Volume $\left[\AA^{3}\right]$ & 2315.31(19) & $1387.71(15)$ \\
\hline $\mathrm{Z}$ & 4 & 2 \\
\hline Calc. density $\left[\mathrm{Mg} \cdot \mathrm{m}^{-3}\right]$ & 1.274 & 1.329 \\
\hline$\mu\left(\mathrm{Mo}_{\mathrm{K} \alpha}\right)\left[\mathrm{mm}^{-1}\right]$ & 0.385 & 0.338 \\
\hline $\mathrm{F}(000)$ & 936 & 580 \\
\hline $\begin{array}{l}\text { Crystal dimensions } \\
{[\mathrm{mm}]}\end{array}$ & $0.46 \times 0.37 \times 0.19$ & $0.25 \times 0.18 \times 0.05$ \\
\hline Theta range $\left[{ }^{\circ}\right]$ & 2.03 to 25.00 & 1.85 to 25.00 \\
\hline \multirow[t]{3}{*}{ Index ranges } & $-10 \leq \mathrm{h} \leq 10$ & $-13 \leq \mathrm{h} \leq 13$ \\
\hline & $-13 \leq \mathrm{k} \leq 13$ & $-14 \leq \mathrm{k} \leq 14$ \\
\hline & $-28 \leq 1 \leq 28$ & $-14 \leq 1 \leq 14$ \\
\hline Reflections collected & 28128 & 16509 \\
\hline Independent reflections & $4090[$ Rint $=0.0252]$ & $4895[$ Rint $=0.0323]$ \\
\hline $\begin{array}{l}\text { Data/Restraints/Paramet } \\
\text { er }\end{array}$ & 4090 / 0 / 261 & 4895 / 0 / 335 \\
\hline Goodness-of-fit on $\mathrm{F}^{2}$ & 1.050 & 1.044 \\
\hline $\begin{array}{l}\text { Final } R \text { indices } \\
{[\mathrm{I}>2 \operatorname{sigma}(\mathrm{I})]}\end{array}$ & $\begin{array}{l}R 1=0.0190 \\
\mathrm{w} R 2=0.0499\end{array}$ & $\begin{array}{l}R 1=0.0379 \\
\mathrm{w} R 2=0.0925\end{array}$ \\
\hline$R$ indices (all data) & $\begin{array}{l}\mathrm{R} 1=0.0198 \\
\mathrm{wR} 2=0.0504\end{array}$ & $\begin{array}{l}\mathrm{R} 1=0.0585 \\
\mathrm{wR} 2=0.1000\end{array}$ \\
\hline Flack parameter & $0.01(4)$ & - \\
\hline $\begin{array}{l}\text { Largest diff. peak and } \\
\text { hole hole }\left[\mathrm{e} \cdot \AA^{-3}\right]\end{array}$ & 0.263 and -0.149 & 0.685 and -0.362 \\
\hline
\end{tabular}


Table S1c. Data collection and structure refinement details for compounds $\mathbf{2 a}, \mathbf{2 b}$ and $\mathbf{2 c}$.

\begin{tabular}{|c|c|c|c|}
\hline Compound & $2 \mathbf{a}$ & $2 \mathbf{b}$ & $2 \mathrm{c}$ \\
\hline CCDC No. & CCDC 1432205 & CCDC 1432206 & CCDC 1432207 \\
\hline Formula & $\mathrm{C}_{53} \mathrm{H}_{49} \mathrm{ClOP}_{2} \mathrm{PdSSi}$ & $\mathrm{C}_{56} \mathrm{H}_{49} \mathrm{IP}_{2} \mathrm{PdSSi}$ & $\mathrm{C}_{34} \mathrm{H}_{35} \mathrm{ClP}_{2} \mathrm{PdSSi}$ \\
\hline $\begin{array}{l}\text { Formula weight } \\
{\left[\mathrm{g} \cdot \mathrm{mol}^{-1}\right]}\end{array}$ & 965.86 & 1077.34 & 707.56 \\
\hline Temperature $[\mathrm{K}]$ & $100(2)$ & $100(2)$ & $100(2)$ \\
\hline Wave length $[\AA]$ & 0.71073 & 0.71073 & 0.71073 \\
\hline Crystal system & monoclinic & monoclinic & triclinic \\
\hline Space group & $P 2{ }_{1} / \mathrm{c}(12)$ & $P 2_{1} / \mathrm{n}(12)$ & $P-1(2)$ \\
\hline $\mathrm{a}[\AA]$ & $12.3157(5)$ & $14.5364(6)$ & $11.989(5)$ \\
\hline $\mathrm{b}[\AA]$ & $18.9079(9)$ & $22.5509(9)$ & $12.008(5)$ \\
\hline c $[\AA ̊]$ & $19.9986(9)$ & $14.6238(6)$ & $13.579(6)$ \\
\hline$\alpha\left[^{\circ}\right]$ & 90 & 90 & $81.286(14)$ \\
\hline$\beta\left[{ }^{\circ}\right]$ & $101.028(1)$ & $94.143(1)$ & $66.326(13)$ \\
\hline$\gamma\left[{ }^{\circ}\right]$ & 90 & 90 & $65.501(13)$ \\
\hline Volume $\left[\AA^{3}\right]$ & $4571.0(4)$ & $4781.3(3)$ & $1628.9(12)$ \\
\hline $\mathrm{Z}$ & 4 & 4 & 2 \\
\hline Calc. density $\left[\mathrm{Mg} \cdot \mathrm{m}^{-3}\right]$ & 1.404 & 1.497 & 1.443 \\
\hline$\mu\left(\mathrm{Mo}_{\mathrm{K} \alpha}\right)\left[\mathrm{mm}^{-1}\right]$ & 0.645 & 1.207 & 0.873 \\
\hline $\mathrm{F}(000)$ & 1992 & 2176 & 724 \\
\hline $\begin{array}{l}\text { Crystal dimensions } \\
{[\mathrm{mm}]}\end{array}$ & $0.42 \times 0.17 \times 0.14$ & $0.27 \times 0.23 \times 0.10$ & $0.27 \times 0.21 \times 0.04$ \\
\hline Theta range $\left[{ }^{\circ}\right]$ & 2.10 to 26.77 & 1.66 to 26.42 & 3.04 to 25.00 \\
\hline \multirow[t]{3}{*}{ Index ranges } & $-15 \leq \mathrm{h} \leq 15$ & $-18 \leq \mathrm{h} \leq 18$ & $-13 \leq \mathrm{h} \leq 14$ \\
\hline & $-23 \leq \mathrm{k} \leq 23$ & $-28 \leq \mathrm{k} \leq 28$ & $-14 \leq \mathrm{k} \leq 14$ \\
\hline & $-25 \leq 1 \leq 25$ & $-18 \leq 1 \leq 18$ & $-16 \leq 1 \leq 16$ \\
\hline Reflections collected & 46331 & 63476 & 12861 \\
\hline Independent reflections & $9628[$ Rint $=0.0406]$ & $9795[$ Rint $=0.0537]$ & $5662[$ Rint $=0.0545]$ \\
\hline $\begin{array}{l}\text { Data/Restraints/Paramet } \\
\text { er }\end{array}$ & $9628 / 0 / 555$ & 9795 / 0 / 560 & 5662 / 0 / 364 \\
\hline Goodness-of-fit on $\mathrm{F}^{2}$ & 1.032 & 1.017 & 1.018 \\
\hline $\begin{array}{l}\text { Final } R \text { indices } \\
{[I>2 \operatorname{sigma}(I)]}\end{array}$ & $\begin{array}{l}R 1=0.0256 \\
\mathrm{w} R 2=0.0602\end{array}$ & $\begin{array}{l}R 1=0.0264 \\
\mathrm{w} R 2=0.0490\end{array}$ & $\begin{array}{l}R 1=0.0394 \\
\mathrm{w} R 2=0.0796\end{array}$ \\
\hline$R$ indices (all data) & $\begin{array}{l}\mathrm{R} 1=0.0331 \\
\mathrm{wR} 2=0.0641\end{array}$ & $\begin{array}{l}\mathrm{R} 1=0.0380 \\
\mathrm{wR} 2=0.0531\end{array}$ & $\begin{array}{l}\mathrm{R} 1=0.0581 \\
\mathrm{wR} 2=0.0886\end{array}$ \\
\hline $\begin{array}{l}\text { Largest diff. peak and } \\
\text { hole hole }\left[\mathrm{e} \cdot \AA^{-3}\right]\end{array}$ & 0.738 and -0.595 & 0.425 and -0.418 & 0.531 and -0.739 \\
\hline
\end{tabular}


Table S1d. Data collection and structure refinement details for compounds $\mathbf{2 d}, \mathbf{2 e}$ and $\mathbf{2 f}$.

\begin{tabular}{|c|c|c|c|}
\hline Compound & 2d & $2 e$ & $2 f$ \\
\hline CCDC No. & CCDC 1432208 & CCDC 1432209 & CCDC 1432210 \\
\hline Formula & $\mathrm{C}_{34} \mathrm{H}_{35} \mathrm{IP}_{2} \mathrm{PdSSi}$ & $\mathrm{C}_{41} \mathrm{H}_{42} \mathrm{ClNP}_{2} \mathrm{PdSSi}$ & $\mathrm{C}_{50} \mathrm{H}_{43} \mathrm{ClOP}_{2} \mathrm{PdSSi}$ \\
\hline $\begin{array}{l}\text { Formula weight } \\
{\left[\mathrm{g} \cdot \mathrm{mol}^{-1}\right]}\end{array}$ & 799.01 & 812.70 & 923.78 \\
\hline Temperature $[\mathrm{K}]$ & $100(2)$ & $100(2)$ & $100(2)$ \\
\hline Wave length $[\AA]$ & 0.71073 & 0.71073 & 0.71073 \\
\hline Crystal system & triclinic & monoclinic & triclinic \\
\hline Space group & $P-1(2)$ & $P 2_{1} / \mathrm{c}(12)$ & $P-1(2)$ \\
\hline $\mathrm{a}[\AA]$ & $10.6034(3)$ & $14.3162(6)$ & $10.0666(5)$ \\
\hline $\mathrm{b}[\AA]$ & $12.6786(4)$ & $14.1803(6)$ & $13.6344(7)$ \\
\hline c $[\AA]$ & $13.9842(4)$ & $19.2993(9)$ & $17.0318(9)$ \\
\hline$\alpha\left[^{\circ}\right]$ & $89.381(1)$ & 90 & $75.168(2)$ \\
\hline$\beta\left[{ }^{\circ}\right]$ & $74.260(1)$ & $101.057(2)$ & $85.252(2)$ \\
\hline$\gamma\left[{ }^{\circ}\right]$ & $68.635(1)$ & 90 & $80.632(2)$ \\
\hline Volume $\left[\AA^{3}\right]$ & 1676.99(9) & $3845.2(3)$ & 2194.32(19) \\
\hline $\mathrm{Z}$ & 2 & 4 & 2 \\
\hline Calc. density $\left[\mathrm{Mg} \cdot \mathrm{m}^{-3}\right]$ & 1.582 & 1.404 & 1.398 \\
\hline$\mu\left(\mathrm{Mo}_{\mathrm{K} \alpha}\right)\left[\mathrm{mm}^{-1}\right]$ & 1.689 & 0.751 & 0.668 \\
\hline $\mathrm{F}(000)$ & 796 & 1672 & 948 \\
\hline $\begin{array}{l}\text { Crystal dimensions } \\
{[\mathrm{mm}]}\end{array}$ & $0.24 \times 0.16 \times 0.13$ & $0.21 \times 0.14 \times 0.07$ & $0.20 \times 0.13 \times 0.10$ \\
\hline Theta range $\left[{ }^{\circ}\right]$ & 2.92 to 26.44 & 1.45 to 25.00 & 1.26 to 25.00 \\
\hline \multirow[t]{3}{*}{ Index ranges } & $-13 \leq \mathrm{h} \leq 13$ & $-17 \leq \mathrm{h} \leq 17$ & $-11 \leq \mathrm{h} \leq 11$ \\
\hline & $-15 \leq \mathrm{k} \leq 15$ & $-16 \leq \mathrm{k} \leq 16$ & $-16 \leq \mathrm{k} \leq 16$ \\
\hline & $-17 \leq 1 \leq 17$ & $-22 \leq 1 \leq 22$ & $-20 \leq 1 \leq 20$ \\
\hline Reflections collected & 22547 & 45827 & 26489 \\
\hline Independent reflections & $6903[$ Rint $=0.0434]$ & $6759[$ Rint $=0.0558]$ & $7712[$ Rint $=0.0542]$ \\
\hline $\begin{array}{l}\text { Data/Restraints/Paramet } \\
\text { er }\end{array}$ & $6903 / 0$ / 364 & $6759 / 0 / 437$ & $7712 / 0 / 515$ \\
\hline Goodness-of-fit on $\mathrm{F}^{2}$ & 1.017 & 1.021 & 1.017 \\
\hline $\begin{array}{l}\text { Final } R \text { indices } \\
{[I>2 \operatorname{sigma}(I)]}\end{array}$ & $\begin{array}{l}R 1=0.0312 \\
\mathrm{w} R 2=0.0568\end{array}$ & $\begin{array}{l}R 1=0.0336 \\
\mathrm{w} R 2=0.0726\end{array}$ & $\begin{array}{l}R 1=0.0355 \\
\mathrm{w} R 2=0.0682\end{array}$ \\
\hline$R$ indices (all data) & $\begin{array}{l}\mathrm{R} 1=0.0461 \\
\mathrm{wR} 2=0.0626\end{array}$ & $\begin{array}{l}\mathrm{R} 1=0.511 \\
\mathrm{wR} 2=0.0796\end{array}$ & $\begin{array}{l}\mathrm{R} 1=0.0539 \\
\mathrm{wR} 2=0.0759\end{array}$ \\
\hline $\begin{array}{l}\text { Largest diff. peak and } \\
\text { hole hole }\left[\mathrm{e} \cdot \AA^{-3}\right]\end{array}$ & 0.322 and -0.473 & 0.748 and -0.678 & 0.447 and -0.633 \\
\hline
\end{tabular}


Table S1e. Data collection and structure refinement details for compounds $\mathbf{3}$ and $\mathbf{5}$.

\begin{tabular}{|c|c|c|}
\hline Compound & 3 & 5 \\
\hline CCDC No. & CCDC 1432211 & CCDC 1432212 \\
\hline Formula & $\mathrm{C}_{98} \mathrm{H}_{80} \mathrm{P}_{4} \mathrm{Pd}_{2} \mathrm{~S}_{2} \mathrm{Si}_{2}$ & $\mathrm{C}_{38} \mathrm{H}_{44} \mathrm{OP}_{2} \mathrm{PdSSi}$ \\
\hline $\begin{array}{l}\text { Formula weight } \\
{\left[\mathrm{g} \cdot \mathrm{mol}^{-1}\right]}\end{array}$ & 1714.60 & 745.22 \\
\hline Temperature $[\mathrm{K}]$ & $100(2)$ & $100(2)$ \\
\hline Wave length $[\AA]$ & 0.71073 & 0.71073 \\
\hline Crystal system & monoclinic & triclinic \\
\hline Space group & $P 2_{1} / \mathrm{n}(14)$ & $P-1(2)$ \\
\hline $\mathrm{a}[\AA]$ & $25.8179(13)$ & $10.6786(5)$ \\
\hline $\mathrm{b}[\AA ̊ \cap]$ & $11.4035(6)$ & $11.8158(5)$ \\
\hline c $[\AA]$ & $27.4898(14)$ & $14.7036(7)$ \\
\hline$\alpha\left[^{\circ}\right]$ & 90 & $81.058(2)$ \\
\hline$\beta\left[^{\circ}\right]$ & $93.816(1)$ & $81.789(2)$ \\
\hline$\gamma\left[^{\circ}\right]$ & 90 & $78.133(2)$ \\
\hline Volume $\left[\AA^{3}\right]$ & $8075.4(7)$ & $1781.70(14)$ \\
\hline $\mathrm{Z}$ & 2 & 2 \\
\hline Calc. density $\left[\mathrm{Mg} \cdot \mathrm{m}^{-3}\right]$ & 1.410 & 1.389 \\
\hline$\mu\left(\mathrm{Mo}_{\mathrm{K} \alpha}\right)\left[\mathrm{mm}^{-1}\right]$ & 0.655 & 0.732 \\
\hline $\mathrm{F}(000)$ & 3520 & 772 \\
\hline $\begin{array}{l}\text { Crystal dimensions } \\
{[\mathrm{mm}]}\end{array}$ & $0.32 \times 0.22 \times 0.12$ & $0.33 \times 0.18 \times 0.07$ \\
\hline Theta range $\left[{ }^{\circ}\right]$ & 1.05 to 25.00 & 1.41 to 25.00 \\
\hline \multirow[t]{3}{*}{ Index ranges } & $-30 \leq \mathrm{h} \leq 30$ & $-12 \leq \mathrm{h} \leq 12$ \\
\hline & $-13 \leq \mathrm{k} \leq 13$ & $-14 \leq \mathrm{k} \leq 14$ \\
\hline & $-32 \leq 1 \leq 32$ & $-17 \leq 1 \leq 17$ \\
\hline Reflections collected & 96001 & 21374 \\
\hline Independent reflections & $14233[$ Rint $=0.0393]$ & $6275[$ Rint $=0.0411]$ \\
\hline $\begin{array}{l}\text { Data/Restraints/Paramet } \\
\text { er }\end{array}$ & 14233 / 0 / 992 & $6275 / 0 / 413$ \\
\hline Goodness-of-fit on $\mathrm{F}^{2}$ & 1.032 & 1.029 \\
\hline $\begin{array}{l}\text { Final } R \text { indices } \\
{[I>2 \operatorname{sigma}(\mathrm{I})]}\end{array}$ & $\begin{array}{l}R 1=0.0277 \\
\mathrm{w} R 2=0.0663\end{array}$ & $\begin{array}{l}R 1=0.0302 \\
\mathrm{w} R 2=0.0620\end{array}$ \\
\hline$R$ indices (all data) & $\begin{array}{l}\mathrm{R} 1=0.0356 \\
\mathrm{wR} 2=0.0705\end{array}$ & $\begin{array}{l}\mathrm{R} 1=0.0448 \\
\mathrm{wR} 2=0.0669\end{array}$ \\
\hline $\begin{array}{l}\text { Largest diff. peak and } \\
\text { hole hole }\left[\mathrm{e} \cdot \AA^{-3}\right]\end{array}$ & 1.147 and -0.515 & 0.514 and -0.448 \\
\hline
\end{tabular}




\subsection{Crystal Structure Determination of 1a}

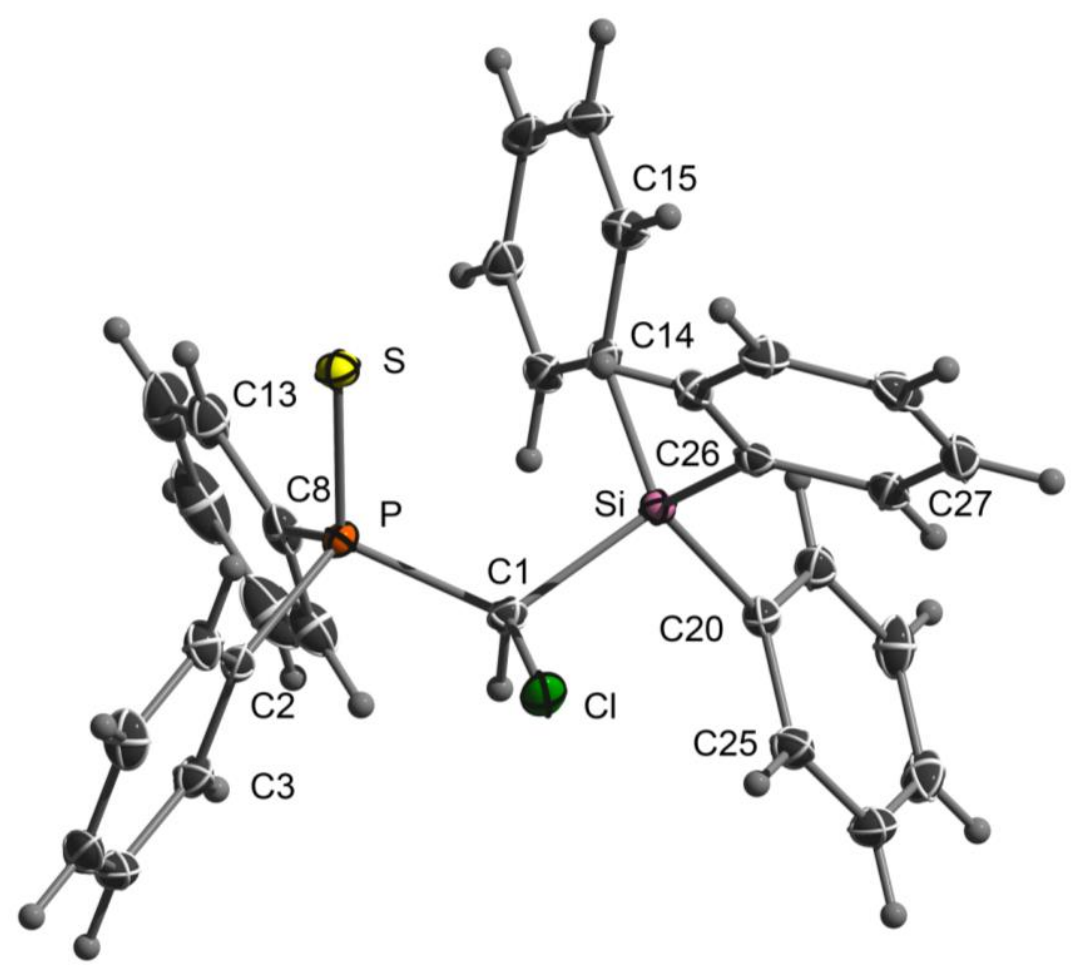

Figure S43. ORTEP Plot of phosphine sulfide 1a. Ellipsoids are drawn at the 50\% probability level

Table S2. Atomic coordinates $\left(\times 10^{4}\right)$ and equivalent isotropic displacement parameters $\left(\AA^{2} \times 10^{3}\right)$ for phosphine sulfide 1a. $\mathrm{U}(\mathrm{eq})$ is defined as one third of the trace of the orthogonalized $\mathrm{U}^{\mathrm{ij}}$ tensor.

\begin{tabular}{lcccc}
\hline & $\mathrm{X}$ & $\mathrm{y}$ & $\mathrm{Z}$ & $\mathrm{U}(\mathrm{eq})$ \\
\hline $\mathrm{Cl}(1)$ & $1513(1)$ & $1974(1)$ & $226(1)$ & $20(1)$ \\
$\mathrm{C}(1)$ & $3180(2)$ & $1966(1)$ & $-125(2)$ & $13(1)$ \\
$\mathrm{S}(1)$ & $5498(1)$ & $2301(1)$ & $2356(1)$ & $18(1)$ \\
$\mathrm{P}(1)$ & $4420(1)$ & $2520(1)$ & $751(1)$ & $12(1)$ \\
$\mathrm{Si}(1)$ & $3889(1)$ & $1193(1)$ & $-56(1)$ & $12(1)$ \\
$\mathrm{C}(2)$ & $3238(2)$ & $3134(1)$ & $659(2)$ & $14(1)$ \\
$\mathrm{C}(3)$ & $2719(2)$ & $3463(1)$ & $-344(2)$ & $18(1)$ \\
$\mathrm{C}(4)$ & $1803(2)$ & $3923(1)$ & $-390(2)$ & $22(1)$ \\
$\mathrm{C}(5)$ & $1400(2)$ & $4058(1)$ & $560(2)$ & $24(1)$ \\
$\mathrm{C}(6)$ & $1922(2)$ & $3735(1)$ & $1564(2)$ & $24(1)$ \\
$\mathrm{C}(7)$ & $2840(2)$ & $3274(1)$ & $1618(2)$ & $19(1)$ \\
$\mathrm{C}(8)$ & $5617(2)$ & $2697(1)$ & $-64(2)$ & $17(1)$ \\
$\mathrm{C}(9)$ & $5098(2)$ & $2702(1)$ & $-1286(2)$ & $25(1)$ \\
$\mathrm{C}(10)$ & $6079(3)$ & $2811(1)$ & $-1867(2)$ & $36(1)$ \\
$\mathrm{C}(11)$ & $7545(3)$ & $2919(1)$ & $-1253(3)$ & $41(1)$ \\
$\mathrm{C}(12)$ & $8060(3)$ & $2933(1)$ & $-53(3)$ & $38(1)$ \\
$\mathrm{C}(14)$ & $5953(2)$ & $1163(1)$ & $410(2)$ & $14(1)$ \\
\hline
\end{tabular}




\begin{tabular}{rrrrr}
$\mathrm{C}(13)$ & $7091(2)$ & $2821(1)$ & $545(2)$ & $26(1)$ \\
$\mathrm{C}(15)$ & $6810(2)$ & $895(1)$ & $1445(2)$ & $18(1)$ \\
$\mathrm{C}(16)$ & $8346(2)$ & $870(1)$ & $1778(2)$ & $22(1)$ \\
$\mathrm{C}(17)$ & $9054(2)$ & $1112(1)$ & $1082(2)$ & $21(1)$ \\
$\mathrm{C}(18)$ & $8222(2)$ & $1375(1)$ & $46(2)$ & $19(1)$ \\
$\mathrm{C}(19)$ & $6696(2)$ & $1398(1)$ & $-284(2)$ & $17(1)$ \\
$\mathrm{C}(20)$ & $3082(2)$ & $914(1)$ & $-1585(2)$ & $15(1)$ \\
$\mathrm{C}(21)$ & $3954(2)$ & $600(1)$ & $-2077(2)$ & $19(1)$ \\
$\mathrm{C}(22)$ & $3356(2)$ & $384(1)$ & $-3198(2)$ & $24(1)$ \\
$\mathrm{C}(23)$ & $1874(2)$ & $471(1)$ & $-3845(2)$ & $26(1)$ \\
$\mathrm{C}(24)$ & $985(2)$ & $773(1)$ & $-3373(2)$ & $26(1)$ \\
$\mathrm{C}(25)$ & $1587(2)$ & $997(1)$ & $-2256(2)$ & $21(1)$ \\
$\mathrm{C}(26)$ & $3186(2)$ & $769(1)$ & $938(2)$ & $15(1)$ \\
$\mathrm{C}(27)$ & $2322(2)$ & $282(1)$ & $546(2)$ & $18(1)$ \\
$\mathrm{C}(28)$ & $1768(2)$ & $-23(1)$ & $1277(2)$ & $22(1)$ \\
$\mathrm{C}(29)$ & $2068(2)$ & $150(1)$ & $2417(2)$ & $21(1)$ \\
$\mathrm{C}(30)$ & $2934(2)$ & $627(1)$ & $2830(2)$ & $21(1)$ \\
$\mathrm{C}(31)$ & $3487(2)$ & $932(1)$ & $2101(2)$ & $18(1)$ \\
\hline
\end{tabular}

Table S3. Anisotropic displacement parameters $\left(\AA^{2} \times 10^{3}\right)$ for phosphine sulfide 1a. The anisotropic displacement factor exponent takes the form: $-2 \pi^{2}\left[\mathrm{~h}^{2} \mathrm{a} \cdot{ }^{2} \mathrm{U}^{11}+\ldots+2 \mathrm{~h} \mathrm{k} \mathrm{a} \cdot \mathrm{b} \cdot \mathrm{U}^{12}\right]$.

\begin{tabular}{ccccccc}
\hline & $\mathrm{U}^{11}$ & $\mathrm{U}^{22}$ & $\mathrm{U}^{22}$ & $\mathrm{U}^{23}$ & $\mathrm{U}^{13}$ & $\mathrm{U}^{12}$ \\
\hline $\mathrm{Cl}(1)$ & $15(1)$ & $20(1)$ & $26(1)$ & $-1(1)$ & $9(1)$ & $0(1)$ \\
$\mathrm{C}(1)$ & $10(1)$ & $14(1)$ & $14(1)$ & $0(1)$ & $4(1)$ & $0(1)$ \\
$\mathrm{S}(1)$ & $18(1)$ & $20(1)$ & $13(1)$ & $1(1)$ & $2(1)$ & $-1(1)$ \\
$\mathrm{P}(1)$ & $12(1)$ & $13(1)$ & $12(1)$ & $0(1)$ & $4(1)$ & $-1(1)$ \\
$\mathrm{Si}(1)$ & $10(1)$ & $13(1)$ & $13(1)$ & $-1(1)$ & $3(1)$ & $0(1)$ \\
$\mathrm{C}(2)$ & $13(1)$ & $13(1)$ & $16(1)$ & $-3(1)$ & $4(1)$ & $-2(1)$ \\
$\mathrm{C}(3)$ & $18(1)$ & $19(1)$ & $14(1)$ & $-4(1)$ & $2(1)$ & $0(1)$ \\
$\mathrm{C}(4)$ & $23(1)$ & $20(1)$ & $18(1)$ & $0(1)$ & $-1(1)$ & $3(1)$ \\
$\mathrm{C}(5)$ & $19(1)$ & $18(1)$ & $31(1)$ & $-6(1)$ & $4(1)$ & $4(1)$ \\
$\mathrm{C}(6)$ & $28(1)$ & $22(1)$ & $27(1)$ & $-6(1)$ & $16(1)$ & $-1(1)$ \\
$\mathrm{C}(7)$ & $22(1)$ & $16(1)$ & $19(1)$ & $0(1)$ & $9(1)$ & $-1(1)$ \\
$\mathrm{C}(8)$ & $20(1)$ & $12(1)$ & $24(1)$ & $1(1)$ & $13(1)$ & $0(1)$ \\
$\mathrm{C}(9)$ & $34(1)$ & $20(1)$ & $25(1)$ & $4(1)$ & $17(1)$ & $5(1)$ \\
$\mathrm{C}(10)$ & $59(2)$ & $26(1)$ & $40(1)$ & $10(1)$ & $37(1)$ & $11(1)$ \\
$\mathrm{C}(11)$ & $56(2)$ & $24(1)$ & $67(2)$ & $11(1)$ & $51(2)$ & $6(1)$ \\
$\mathrm{C}(12)$ & $26(1)$ & $25(1)$ & $71(2)$ & $3(1)$ & $29(1)$ & $-3(1)$ \\
$\mathrm{C}(14)$ & $14(1)$ & $13(1)$ & $16(1)$ & $-4(1)$ & $4(1)$ & $1(1)$
\end{tabular}




\begin{tabular}{lllllll}
$\mathrm{C}(13)$ & $22(1)$ & $20(1)$ & $39(1)$ & $1(1)$ & $14(1)$ & $-3(1)$ \\
$\mathrm{C}(15)$ & $18(1)$ & $19(1)$ & $17(1)$ & $1(1)$ & $6(1)$ & $3(1)$ \\
$\mathrm{C}(16)$ & $17(1)$ & $24(1)$ & $20(1)$ & $-1(1)$ & $0(1)$ & $5(1)$ \\
$\mathrm{C}(17)$ & $11(1)$ & $20(1)$ & $31(1)$ & $-5(1)$ & $4(1)$ & $1(1)$ \\
$\mathrm{C}(18)$ & $16(1)$ & $17(1)$ & $26(1)$ & $-1(1)$ & $8(1)$ & $-1(1)$ \\
$\mathrm{C}(19)$ & $16(1)$ & $15(1)$ & $19(1)$ & $-1(1)$ & $5(1)$ & $2(1)$ \\
$\mathrm{C}(20)$ & $16(1)$ & $13(1)$ & $16(1)$ & $0(1)$ & $5(1)$ & $-1(1)$ \\
$\mathrm{C}(21)$ & $17(1)$ & $17(1)$ & $23(1)$ & $-2(1)$ & $7(1)$ & $-1(1)$ \\
$\mathrm{C}(22)$ & $31(1)$ & $20(1)$ & $26(1)$ & $-7(1)$ & $16(1)$ & $-5(1)$ \\
$\mathrm{C}(23)$ & $37(1)$ & $21(1)$ & $15(1)$ & $-4(1)$ & $5(1)$ & $-5(1)$ \\
$\mathrm{C}(24)$ & $25(1)$ & $23(1)$ & $21(1)$ & $-1(1)$ & $-3(1)$ & $4(1)$ \\
$\mathrm{C}(25)$ & $20(1)$ & $20(1)$ & $21(1)$ & $-3(1)$ & $3(1)$ & $4(1)$ \\
$\mathrm{C}(26)$ & $12(1)$ & $14(1)$ & $18(1)$ & $2(1)$ & $4(1)$ & $4(1)$ \\
$\mathrm{C}(27)$ & $15(1)$ & $15(1)$ & $22(1)$ & $-1(1)$ & $5(1)$ & $2(1)$ \\
$\mathrm{C}(28)$ & $18(1)$ & $14(1)$ & $32(1)$ & $1(1)$ & $8(1)$ & $-1(1)$ \\
$\mathrm{C}(29)$ & $18(1)$ & $20(1)$ & $27(1)$ & $10(1)$ & $10(1)$ & $3(1)$ \\
$\mathrm{C}(30)$ & $20(1)$ & $23(1)$ & $18(1)$ & $4(1)$ & $5(1)$ & $2(1)$ \\
$\mathrm{C}(31)$ & $16(1)$ & $17(1)$ & $18(1)$ & $2(1)$ & $4(1)$ & $-1(1)$ \\
\hline
\end{tabular}

\subsection{Crystal Structure Determination of $\mathbf{1 b}$}

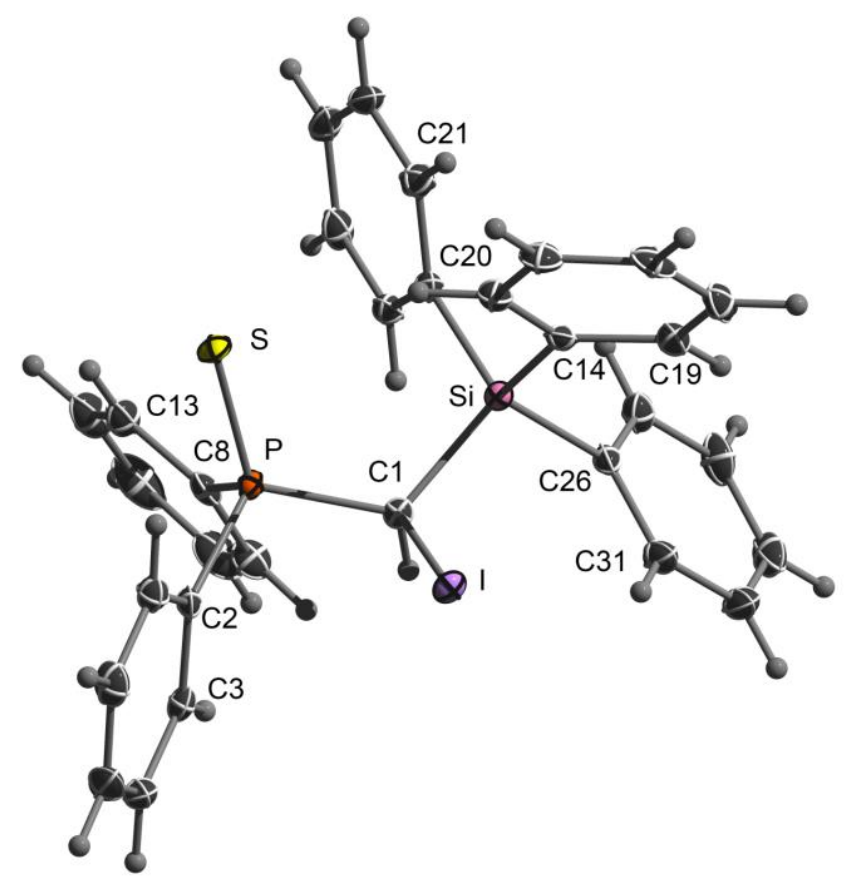

Figure S44. ORTEP Plot of phosphine sulfide 1b. Ellipsoids are drawn at the 50\% probability level 
Table S4. Atomic coordinates $\left(\mathrm{x} 10^{4}\right)$ and equivalent isotropic displacement parameters $\left(\AA^{2} \times 10^{3}\right)$ for phosphine sulfide 1b. $U(\mathrm{eq})$ is defined as one third of the trace of the orthogonalized $\mathrm{U}^{\mathrm{ij}}$ tensor.

\begin{tabular}{|c|c|c|c|c|}
\hline & $\mathrm{X}$ & $\mathrm{y}$ & $\mathrm{Z}$ & $\mathrm{U}(\mathrm{eq})$ \\
\hline $\mathrm{I}(1)$ & $8742(1)$ & $1987(1)$ & $4837(1)$ & $14(1)$ \\
\hline $\mathrm{C}(1)$ & $6772(2)$ & $1963(1)$ & $5147(2)$ & 11(1) \\
\hline $\mathrm{S}(1)$ & $4471(1)$ & $2267(1)$ & $2660(1)$ & $15(1)$ \\
\hline $\mathrm{P}(1)$ & $5540(1)$ & $2510(1)$ & $4250(1)$ & 11(1) \\
\hline $\mathrm{Si}(1)$ & $6130(1)$ & $1178(1)$ & $5058(1)$ & $11(1)$ \\
\hline $\mathrm{C}(2)$ & $6638(2)$ & $3138(1)$ & $4315(2)$ & $13(1)$ \\
\hline $\mathrm{C}(3)$ & $6960(2)$ & $3277(1)$ & $3350(2)$ & $18(1)$ \\
\hline $\mathrm{C}(4)$ & $7870(2)$ & $3731(1)$ & $3398(2)$ & $23(1)$ \\
\hline$C(5)$ & $8452(2)$ & $4049(1)$ & $4403(2)$ & $26(1)$ \\
\hline$C(6)$ & $8119(2)$ & $3919(1)$ & $5353(2)$ & $23(1)$ \\
\hline$C(7)$ & $7213(2)$ & $3465(1)$ & $5315(2)$ & $16(1)$ \\
\hline $\mathrm{C}(8)$ & $4404(2)$ & $2695(1)$ & $5039(2)$ & $14(1)$ \\
\hline $\mathrm{C}(9)$ & 4913(2) & 2701(1) & $6241(2)$ & $21(1)$ \\
\hline$C(10)$ & 3992(3) & $2817(1)$ & $6806(2)$ & $29(1)$ \\
\hline $\mathrm{C}(11)$ & $2558(3)$ & 2934(1) & $6173(2)$ & $33(1)$ \\
\hline$C(12)$ & $2054(3)$ & $2950(1)$ & $4975(2)$ & $30(1)$ \\
\hline$C(14)$ & $6785(2)$ & $753(1)$ & $4078(2)$ & $14(1)$ \\
\hline$C(13)$ & $2972(2)$ & $2830(1)$ & $4406(2)$ & $21(1)$ \\
\hline$C(15)$ & $6454(2)$ & $916(1)$ & 2923(2) & $18(1)$ \\
\hline$C(16)$ & $7002(2)$ & $621(1)$ & $2216(2)$ & $21(1)$ \\
\hline $\mathrm{C}(17)$ & $7902(2)$ & $157(1)$ & $2648(2)$ & $21(1)$ \\
\hline $\mathrm{C}(18)$ & $8228(2)$ & $-17(1)$ & $3780(2)$ & $21(1)$ \\
\hline C(19) & $7669(2)$ & $277(1)$ & $4485(2)$ & $18(1)$ \\
\hline$C(20)$ & $4131(2)$ & $1138(1)$ & $4563(2)$ & $14(1)$ \\
\hline $\mathrm{C}(21)$ & $3295(2)$ & $872(1)$ & $3523(2)$ & $18(1)$ \\
\hline $\mathrm{C}(22)$ & $1812(2)$ & $841(1)$ & $3169(2)$ & $22(1)$ \\
\hline$C(23)$ & $1139(2)$ & $1072(1)$ & $3855(2)$ & $24(1)$ \\
\hline $\mathrm{C}(24)$ & $1951(2)$ & 1331(1) & $4893(2)$ & $21(1)$ \\
\hline$C(25)$ & $3427(2)$ & $1365(1)$ & $5242(2)$ & $17(1)$ \\
\hline$C(26)$ & $6930(2)$ & $897(1)$ & $6584(2)$ & $14(1)$ \\
\hline $\mathrm{C}(27)$ & $6088(2)$ & $607(1)$ & $7080(2)$ & $20(1)$ \\
\hline $\mathrm{C}(28)$ & $6667(3)$ & $401(1)$ & $8205(2)$ & $25(1)$ \\
\hline $\mathrm{C}(29)$ & $8111(3)$ & $473(1)$ & $8861(2)$ & $27(1)$ \\
\hline$C(30)$ & $8973(3)$ & $752(1)$ & $8388(2)$ & $28(1)$ \\
\hline $\mathrm{C}(31)$ & $8390(2)$ & $965(1)$ & $7268(2)$ & $22(1)$ \\
\hline
\end{tabular}


Table S5. Anisotropic displacement parameters $\left(\AA^{2} \times 10^{3}\right)$ for phosphine sulfide 1b. The anisotropic displacement factor exponent takes the form: $-2 \pi^{2}\left[h^{2} a \cdot{ }^{2} U^{11}+\ldots+2 h \mathrm{k} \mathrm{a} \cdot \mathrm{b} \cdot \mathrm{U}^{12}\right]$.

\begin{tabular}{|c|c|c|c|c|c|c|}
\hline & $\mathrm{U}^{11}$ & $\mathrm{U}^{22}$ & $\mathrm{U}^{22}$ & $\mathrm{U}^{23}$ & $\mathrm{U}^{13}$ & $\mathrm{U}^{12}$ \\
\hline $\mathrm{I}(1)$ & $11(1)$ & $17(1)$ & $15(1)$ & $0(1)$ & $6(1)$ & $0(1)$ \\
\hline $\mathrm{C}(1)$ & $11(1)$ & $12(1)$ & $10(1)$ & 1(1) & $5(1)$ & $0(1)$ \\
\hline$S(1)$ & $16(1)$ & $18(1)$ & $9(1)$ & $-1(1)$ & 1(1) & 1(1) \\
\hline $\mathrm{P}(1)$ & $11(1)$ & $12(1)$ & $9(1)$ & $0(1)$ & $3(1)$ & 1(1) \\
\hline $\operatorname{Si}(1)$ & $10(1)$ & $12(1)$ & $11(1)$ & $0(1)$ & $3(1)$ & $0(1)$ \\
\hline $\mathrm{C}(2)$ & $13(1)$ & $10(1)$ & $16(1)$ & $4(1)$ & $4(1)$ & $2(1)$ \\
\hline $\mathrm{C}(3)$ & $21(1)$ & $16(1)$ & $16(1)$ & $2(1)$ & $6(1)$ & $1(1)$ \\
\hline $\mathrm{C}(4)$ & $26(1)$ & $22(1)$ & $24(1)$ & $7(1)$ & $11(1)$ & $0(1)$ \\
\hline $\mathrm{C}(5)$ & $22(1)$ & $18(1)$ & $31(1)$ & $7(1)$ & $4(1)$ & $-7(1)$ \\
\hline $\mathrm{C}(6)$ & $23(1)$ & $17(1)$ & $18(1)$ & $1(1)$ & $-3(1)$ & $-3(1)$ \\
\hline $\mathrm{C}(7)$ & $16(1)$ & $16(1)$ & $13(1)$ & $4(1)$ & $2(1)$ & $3(1)$ \\
\hline $\mathrm{C}(8)$ & $16(1)$ & $9(1)$ & $20(1)$ & $-1(1)$ & $10(1)$ & $0(1)$ \\
\hline $\mathrm{C}(9)$ & $26(1)$ & $20(1)$ & 21(1) & $-3(1)$ & $14(1)$ & $0(1)$ \\
\hline$C(10)$ & $46(2)$ & $24(1)$ & $29(1)$ & $-7(1)$ & $26(1)$ & $-4(1)$ \\
\hline$C(11)$ & $42(2)$ & $22(1)$ & $55(2)$ & $-9(1)$ & $41(1)$ & $-4(1)$ \\
\hline$C(12)$ & $21(1)$ & $21(1)$ & $53(2)$ & $-4(1)$ & $20(1)$ & $1(1)$ \\
\hline $\mathrm{C}(14)$ & $11(1)$ & $12(1)$ & $17(1)$ & $-4(1)$ & $4(1)$ & $-3(1)$ \\
\hline$C(13)$ & $16(1)$ & $18(1)$ & $31(1)$ & $0(1)$ & $10(1)$ & $1(1)$ \\
\hline$C(15)$ & $18(1)$ & $17(1)$ & $17(1)$ & $-2(1)$ & $4(1)$ & 1(1) \\
\hline$C(16)$ & $24(1)$ & $22(1)$ & $16(1)$ & $-6(1)$ & $6(1)$ & $-4(1)$ \\
\hline$C(17)$ & $21(1)$ & $17(1)$ & $29(1)$ & $-12(1)$ & $14(1)$ & $-4(1)$ \\
\hline $\mathrm{C}(18)$ & $19(1)$ & $14(1)$ & 31(1) & $-1(1)$ & $10(1)$ & $3(1)$ \\
\hline C(19) & $17(1)$ & $15(1)$ & $20(1)$ & 1(1) & $6(1)$ & $0(1)$ \\
\hline$C(20)$ & $13(1)$ & $12(1)$ & $16(1)$ & $2(1)$ & $3(1)$ & $0(1)$ \\
\hline$C(21)$ & $18(1)$ & $18(1)$ & $17(1)$ & $1(1)$ & $6(1)$ & $-2(1)$ \\
\hline $\mathrm{C}(22)$ & $17(1)$ & $19(1)$ & $21(1)$ & $2(1)$ & $-2(1)$ & $-5(1)$ \\
\hline$C(23)$ & $10(1)$ & $20(1)$ & $36(1)$ & $5(1)$ & $3(1)$ & $-1(1)$ \\
\hline $\mathrm{C}(24)$ & $16(1)$ & $17(1)$ & $33(1)$ & $2(1)$ & $13(1)$ & $2(1)$ \\
\hline$C(25)$ & $14(1)$ & $13(1)$ & $22(1)$ & $-1(1)$ & $5(1)$ & $-1(1)$ \\
\hline$C(26)$ & $19(1)$ & 11(1) & $13(1)$ & $0(1)$ & $5(1)$ & 1(1) \\
\hline $\mathrm{C}(27)$ & $18(1)$ & $18(1)$ & $24(1)$ & $3(1)$ & $9(1)$ & $4(1)$ \\
\hline $\mathrm{C}(28)$ & $35(1)$ & $20(1)$ & $26(1)$ & $9(1)$ & $17(1)$ & $4(1)$ \\
\hline C(29) & $41(2)$ & $20(1)$ & $15(1)$ & $4(1)$ & $5(1)$ & $3(1)$ \\
\hline $\mathrm{C}(30)$ & $28(1)$ & $22(1)$ & $22(1)$ & $4(1)$ & $-6(1)$ & $-6(1)$ \\
\hline $\mathrm{C}(31)$ & $22(1)$ & $18(1)$ & $21(1)$ & $5(1)$ & $2(1)$ & $-6(1)$ \\
\hline
\end{tabular}




\subsection{Crystal Structure Determination of $\mathbf{1 d}$}

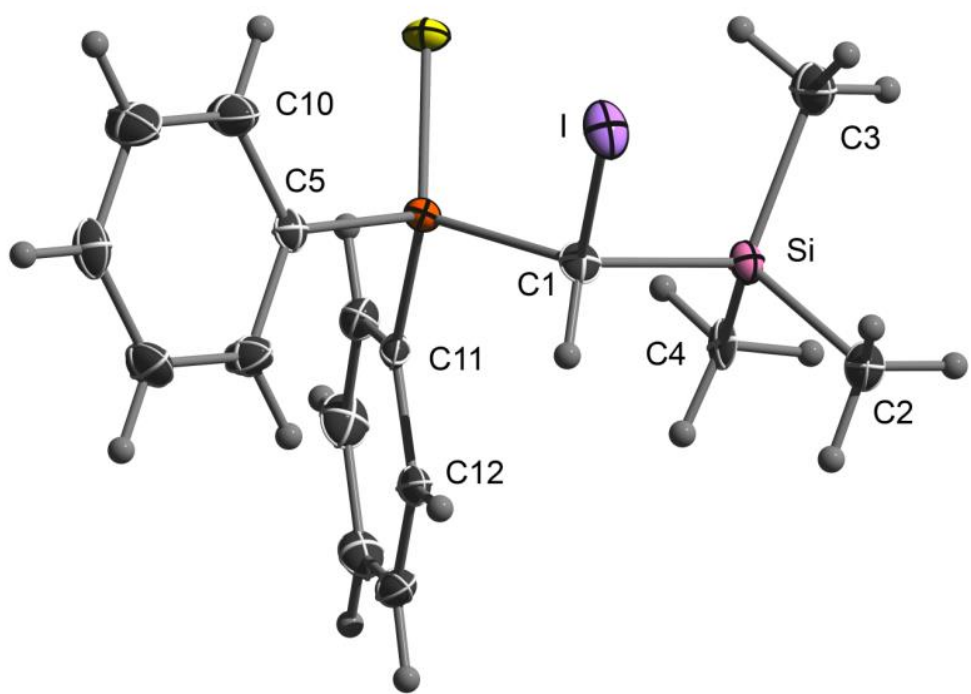

Figure S45. ORTEP Plot of phosphine sulfide 1d. Ellipsoids are drawn at the 50\% probability level

Table S6. Atomic coordinates $\left(\mathrm{x} 10^{4}\right)$ and equivalent isotropic displacement parameters $\left(\AA^{2} \times 10^{3}\right)$ for phosphine sulfide 1d. $\mathrm{U}(\mathrm{eq})$ is defined as one third of the trace of the orthogonalized $\mathrm{U}^{\mathrm{ij}}$ tensor.

\begin{tabular}{|c|c|c|c|c|}
\hline & $\mathrm{X}$ & $\mathrm{y}$ & $\mathrm{Z}$ & $\mathrm{U}(\mathrm{eq})$ \\
\hline $\mathrm{I}(1)$ & $6069(1)$ & $6501(1)$ & $698(1)$ & $22(1)$ \\
\hline $\mathrm{S}(1)$ & $4058(1)$ & $4056(1)$ & $1518(1)$ & $17(1)$ \\
\hline $\mathrm{P}(1)$ & 3891(1) & $5552(1)$ & $1844(1)$ & $11(1)$ \\
\hline $\operatorname{Si}(1)$ & $7669(1)$ & $5863(1)$ & $2606(1)$ & $14(1)$ \\
\hline $\mathrm{C}(1)$ & $5705(3)$ & $6336(2)$ & 1914(2) & $14(1)$ \\
\hline $\mathrm{C}(2)$ & $8985(3)$ & $7061(2)$ & $2751(2)$ & $20(1)$ \\
\hline $\mathrm{C}(3)$ & $8561(3)$ & $4754(2)$ & $2134(2)$ & $24(1)$ \\
\hline $\mathrm{C}(4)$ & $7360(3)$ & $5425(2)$ & $3609(1)$ & $14(1)$ \\
\hline$C(5)$ & $2315(3)$ & $6284(2)$ & $1144(2)$ & $13(1)$ \\
\hline$C(6)$ & $1746(3)$ & $7262(2)$ & $1355(2)$ & $21(1)$ \\
\hline$C(7)$ & $562(3)$ & $7806(2)$ & $805(2)$ & $23(1)$ \\
\hline $\mathrm{C}(8)$ & $-53(3)$ & $7388(2)$ & $44(2)$ & $22(1)$ \\
\hline $\mathrm{C}(9)$ & $521(4)$ & $6431(2)$ & $-174(2)$ & $29(1)$ \\
\hline$C(10)$ & 1701(4) & $5876(2)$ & $378(2)$ & $26(1)$ \\
\hline $\mathrm{C}(11)$ & $3500(3)$ & $5677(2)$ & $2851(2)$ & $13(1)$ \\
\hline $\mathrm{C}(12)$ & $3637(3)$ & $6665(2)$ & $3252(2)$ & $15(1)$ \\
\hline$C(13)$ & $3371(3)$ & $6743(2)$ & $4022(2)$ & $20(1)$ \\
\hline$C(14)$ & $3004(3)$ & $5830(2)$ & $4410(2)$ & $24(1)$ \\
\hline$C(15)$ & $2888(3)$ & $4845(2)$ & $4023(2)$ & $24(1)$ \\
\hline
\end{tabular}




\begin{tabular}{lllll}
$\mathrm{C}(16)$ & $3123(3)$ & $4769(2)$ & $3242(2)$ & $17(1)$ \\
\hline
\end{tabular}

Table S7. Anisotropic displacement parameters $\left(\AA^{2} \times 10^{3}\right)$ for phosphine sulfide 1d. The anisotropic displacement factor exponent takes the form: $-2 \pi^{2}\left[\mathrm{~h}^{2} \mathrm{a} \cdot{ }^{2} \mathrm{U}^{11}+\ldots+2 \mathrm{~h} \mathrm{k} \mathrm{a} \cdot \mathrm{b} \cdot \mathrm{U}^{12}\right]$.

\begin{tabular}{lcccccc}
\hline & $\mathrm{U}^{11}$ & $\mathrm{U}^{22}$ & $\mathrm{U}^{22}$ & $\mathrm{U}^{23}$ & $\mathrm{U}^{13}$ & $\mathrm{U}^{12}$ \\
\hline $\mathrm{I}(1)$ & $18(1)$ & $31(1)$ & $16(1)$ & $9(1)$ & $7(1)$ & $2(1)$ \\
$\mathrm{S}(1)$ & $21(1)$ & $12(1)$ & $18(1)$ & $-2(1)$ & $5(1)$ & $2(1)$ \\
$\mathrm{P}(1)$ & $12(1)$ & $11(1)$ & $12(1)$ & $0(1)$ & $3(1)$ & $1(1)$ \\
$\mathrm{Si}(1)$ & $12(1)$ & $17(1)$ & $14(1)$ & $2(1)$ & $2(1)$ & $1(1)$ \\
$\mathrm{C}(1)$ & $16(1)$ & $17(1)$ & $10(1)$ & $3(1)$ & $6(1)$ & $-1(1)$ \\
$\mathrm{C}(2)$ & $15(1)$ & $23(2)$ & $22(2)$ & $-1(1)$ & $2(1)$ & $0(1)$ \\
$\mathrm{C}(3)$ & $21(2)$ & $26(2)$ & $23(2)$ & $1(1)$ & $0(1)$ & $6(1)$ \\
$\mathrm{C}(4)$ & $9(1)$ & $21(1)$ & $10(1)$ & $6(1)$ & $-2(1)$ & $3(1)$ \\
$\mathrm{C}(5)$ & $12(1)$ & $15(1)$ & $14(1)$ & $2(1)$ & $5(1)$ & $-1(1)$ \\
$\mathrm{C}(6)$ & $24(2)$ & $20(2)$ & $16(2)$ & $-3(1)$ & $-2(1)$ & $3(1)$ \\
$\mathrm{C}(7)$ & $23(2)$ & $18(2)$ & $28(2)$ & $-1(1)$ & $4(1)$ & $7(1)$ \\
$\mathrm{C}(8)$ & $16(2)$ & $28(2)$ & $21(2)$ & $8(1)$ & $2(1)$ & $3(1)$ \\
$\mathrm{C}(9)$ & $32(2)$ & $36(2)$ & $16(2)$ & $-5(1)$ & $-2(1)$ & $11(2)$ \\
$\mathrm{C}(10)$ & $29(2)$ & $26(2)$ & $21(2)$ & $-7(1)$ & $0(1)$ & $7(1)$ \\
$\mathrm{C}(11)$ & $8(1)$ & $18(1)$ & $11(1)$ & $-2(1)$ & $1(1)$ & $-1(1)$ \\
$\mathrm{C}(12)$ & $12(1)$ & $16(1)$ & $16(1)$ & $-1(1)$ & $0(1)$ & $2(1)$ \\
$\mathrm{C}(13)$ & $18(2)$ & $22(2)$ & $18(2)$ & $-6(1)$ & $-1(1)$ & $2(1)$ \\
$\mathrm{C}(14)$ & $20(2)$ & $40(2)$ & $13(1)$ & $-1(1)$ & $5(1)$ & $4(1)$ \\
$\mathrm{C}(15)$ & $23(2)$ & $30(2)$ & $21(2)$ & $4(1)$ & $10(1)$ & $-4(1)$ \\
$\mathrm{C}(16)$ & $15(1)$ & $18(1)$ & $18(1)$ & $-1(1)$ & $3(1)$ & $-2(1)$ \\
\hline & & & & & &
\end{tabular}




\subsection{Crystal Structure Determination of $\mathbf{1 e}$}

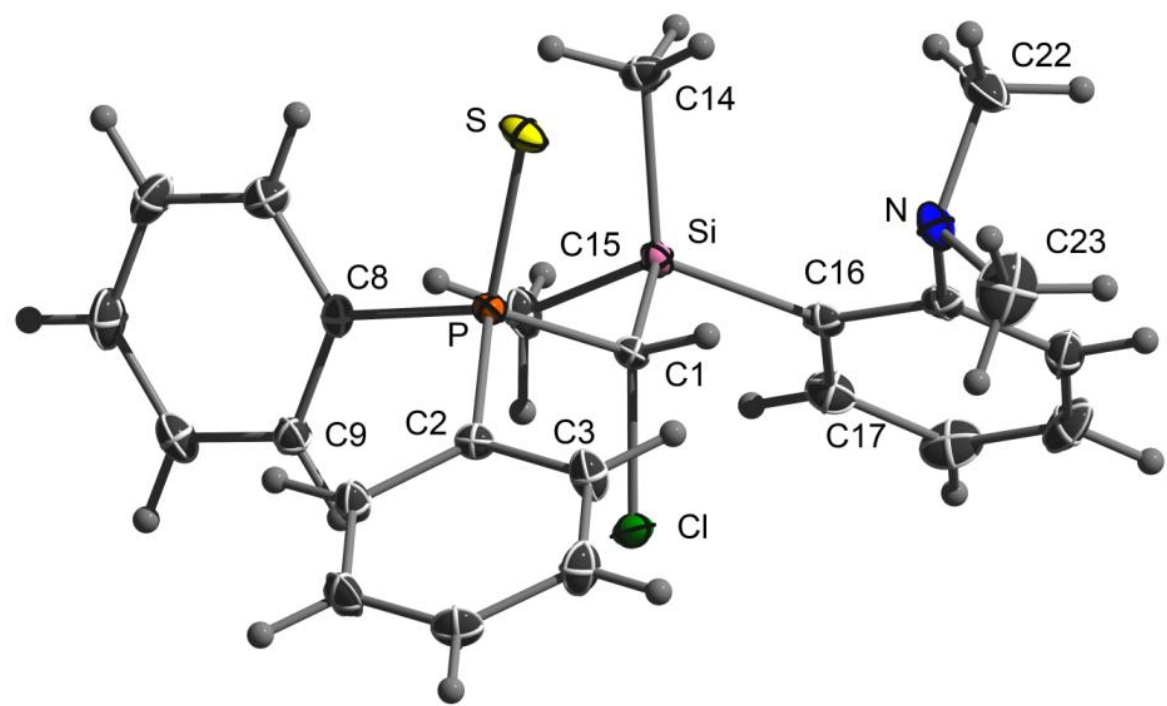

Figure S46. ORTEP Plot of phosphine sulfide 1e. Ellipsoids are drawn at the $50 \%$ probability level

Table S8. Atomic coordinates $\left(\mathrm{x} 10^{4}\right)$ and equivalent isotropic displacement parameters $\left(\AA^{2} \times 10^{3}\right)$ for phosphine sulfide 1e. $\mathrm{U}(\mathrm{eq})$ is defined as one third of the trace of the orthogonalized $\mathrm{U}^{\mathrm{ij}}$ tensor.

\begin{tabular}{|c|c|c|c|c|}
\hline & $\mathrm{X}$ & $\mathrm{y}$ & $\mathrm{Z}$ & $\mathrm{U}(\mathrm{eq})$ \\
\hline $\mathrm{P}(1)$ & 2998(1) & $1176(1)$ & 1661(1) & $10(1)$ \\
\hline $\mathrm{C}(1)$ & $2347(2)$ & $-243(1)$ & $1344(1)$ & $11(1)$ \\
\hline $\mathrm{N}(1)$ & $-737(1)$ & $-995(1)$ & $636(1)$ & $17(1)$ \\
\hline $\mathrm{Cl}(1)$ & $3957(1)$ & $-1181(1)$ & $1138(1)$ & $17(1)$ \\
\hline $\mathrm{Si}(1)$ & $958(1)$ & $-1282(1)$ & $1744(1)$ & $11(1)$ \\
\hline$S(1)$ & $1294(1)$ & $2333(1)$ & $1665(1)$ & $17(1)$ \\
\hline$C(2)$ & $4603(2)$ & $1717(1)$ & $1249(1)$ & $12(1)$ \\
\hline $\mathrm{C}(4)$ & $5556(2)$ & $2331(1)$ & $354(1)$ & $20(1)$ \\
\hline$C(5)$ & $6912(2)$ & $2726(1)$ & $600(1)$ & $19(1)$ \\
\hline$C(6)$ & $7120(2)$ & $2616(2)$ & 1161(1) & $20(1)$ \\
\hline$C(7)$ & $5970(2)$ & $2108(1)$ & $1488(1)$ & $16(1)$ \\
\hline $\mathrm{C}(8)$ & $3758(2)$ & $826(1)$ & $2342(1)$ & $12(1)$ \\
\hline $\mathrm{C}(9)$ & $5059(2)$ & $97(1)$ & $2410(1)$ & $15(1)$ \\
\hline$C(10)$ & $5591(2)$ & $-175(1)$ & $2937(1)$ & $18(1)$ \\
\hline $\mathrm{C}(11)$ & $4823(2)$ & $273(2)$ & $3397(1)$ & $23(1)$ \\
\hline $\mathrm{C}(12)$ & $3532(2)$ & 994(1) & $3334(1)$ & $23(1)$ \\
\hline $\mathrm{C}(13)$ & 2998(2) & $1274(1)$ & $2806(1)$ & $18(1)$ \\
\hline $\mathrm{C}(14)$ & $-690(2)$ & $-402(1)$ & $2030(1)$ & $18(1)$ \\
\hline$C(15)$ & $2017(2)$ & $-2003(1)$ & $2328(1)$ & $17(1)$ \\
\hline$C(16)$ & $418(2)$ & $-2509(1)$ & $1239(1)$ & $12(1)$ \\
\hline
\end{tabular}




\begin{tabular}{rrrrr}
$\mathrm{C}(17)$ & $810(2)$ & $-3727(1)$ & $1342(1)$ & $19(1)$ \\
$\mathrm{C}(18)$ & $495(2)$ & $-4635(1)$ & $961(1)$ & $25(1)$ \\
$\mathrm{C}(19)$ & $-224(2)$ & $-4345(1)$ & $469(1)$ & $25(1)$ \\
$\mathrm{C}(22)$ & $-2395(2)$ & $-820(2)$ & $740(1)$ & $28(1)$ \\
$\mathrm{C}(20)$ & $-638(2)$ & $-3155(1)$ & $354(1)$ & $20(1)$ \\
$\mathrm{C}(23)$ & $-336(3)$ & $-569(2)$ & $83(1)$ & $35(1)$ \\
$\mathrm{C}(21)$ & $-316(2)$ & $-2246(1)$ & $739(1)$ & $13(1)$ \\
$\mathrm{C}(3)$ & $4403(2)$ & $1837(1)$ & $679(1)$ & $18(1)$ \\
\hline
\end{tabular}

Table S9. Anisotropic displacement parameters $\left(\AA^{2} \times 10^{3}\right)$ for phosphine sulfide 1e. The anisotropic displacement factor exponent takes the form: $-2 \pi^{2}\left[\mathrm{~h}^{2} \mathrm{a}^{\cdot 2} \mathrm{U}^{11}+\ldots+2 \mathrm{~h} \mathrm{k} \mathrm{a} \cdot \mathrm{b} \cdot \mathrm{U}^{12}\right]$.

\begin{tabular}{|c|c|c|c|c|c|c|}
\hline & $\mathrm{U}^{11}$ & $\mathrm{U}^{22}$ & $\mathrm{U}^{22}$ & $\mathrm{U}^{23}$ & $\mathrm{U}^{13}$ & $\mathrm{U}^{12}$ \\
\hline $\mathrm{P}(1)$ & $9(1)$ & 11(1) & 11(1) & $0(1)$ & $0(1)$ & $0(1)$ \\
\hline $\mathrm{C}(1)$ & $8(1)$ & $12(1)$ & $12(1)$ & $-1(1)$ & $0(1)$ & $1(1)$ \\
\hline $\mathrm{N}(1)$ & $17(1)$ & $15(1)$ & 19(1) & $3(1)$ & $-5(1)$ & $0(1)$ \\
\hline $\mathrm{Cl}(1)$ & $14(1)$ & $17(1)$ & $19(1)$ & $-5(1)$ & $5(1)$ & $0(1)$ \\
\hline $\mathrm{Si}(1)$ & $10(1)$ & $12(1)$ & $11(1)$ & 1(1) & $0(1)$ & $-1(1)$ \\
\hline $\mathrm{S}(1)$ & $12(1)$ & $14(1)$ & $25(1)$ & $2(1)$ & $-1(1)$ & $3(1)$ \\
\hline $\mathrm{C}(2)$ & 11(1) & $10(1)$ & $14(1)$ & $1(1)$ & $1(1)$ & $0(1)$ \\
\hline $\mathrm{C}(4)$ & $25(1)$ & $22(1)$ & $13(1)$ & $3(1)$ & $-1(1)$ & $-3(1)$ \\
\hline$C(5)$ & $17(1)$ & $20(1)$ & 19(1) & $3(1)$ & $6(1)$ & $-3(1)$ \\
\hline$C(6)$ & $12(1)$ & $27(1)$ & 21(1) & $0(1)$ & $-2(1)$ & $-5(1)$ \\
\hline$C(7)$ & $16(1)$ & $21(1)$ & $12(1)$ & $0(1)$ & $-2(1)$ & $-3(1)$ \\
\hline $\mathrm{C}(8)$ & $13(1)$ & $12(1)$ & 11(1) & $1(1)$ & $-2(1)$ & $-3(1)$ \\
\hline $\mathrm{C}(9)$ & $13(1)$ & $17(1)$ & $14(1)$ & $0(1)$ & $0(1)$ & $-2(1)$ \\
\hline $\mathrm{C}(10)$ & $15(1)$ & 19(1) & $20(1)$ & $4(1)$ & $-5(1)$ & $-2(1)$ \\
\hline $\mathrm{C}(11)$ & $29(1)$ & $27(1)$ & $13(1)$ & $3(1)$ & $-5(1)$ & $-5(1)$ \\
\hline $\mathrm{C}(12)$ & $30(1)$ & $27(1)$ & $13(1)$ & $-4(1)$ & $4(1)$ & $-1(1)$ \\
\hline $\mathrm{C}(13)$ & $19(1)$ & $16(1)$ & $18(1)$ & $-1(1)$ & $2(1)$ & 1(1) \\
\hline $\mathrm{C}(14)$ & $14(1)$ & $18(1)$ & $21(1)$ & $-2(1)$ & $6(1)$ & $-1(1)$ \\
\hline$C(15)$ & $18(1)$ & $21(1)$ & $14(1)$ & $4(1)$ & $-3(1)$ & $-3(1)$ \\
\hline $\mathrm{C}(16)$ & $10(1)$ & $12(1)$ & $15(1)$ & $1(1)$ & 2(1) & $-2(1)$ \\
\hline $\mathrm{C}(17)$ & $18(1)$ & $16(1)$ & $22(1)$ & $4(1)$ & $1(1)$ & $2(1)$ \\
\hline $\mathrm{C}(18)$ & $31(1)$ & 11(1) & $35(1)$ & 1(1) & $6(1)$ & $1(1)$ \\
\hline $\mathrm{C}(19)$ & $34(1)$ & $17(1)$ & $25(1)$ & $-9(1)$ & $4(1)$ & $-6(1)$ \\
\hline $\mathrm{C}(22)$ & $17(1)$ & $25(1)$ & $44(1)$ & $-4(1)$ & $-9(1)$ & $5(1)$ \\
\hline $\mathrm{C}(20)$ & $22(1)$ & $23(1)$ & $15(1)$ & $-3(1)$ & $-1(1)$ & $-4(1)$ \\
\hline $\mathrm{C}(23)$ & $51(1)$ & $29(1)$ & $25(1)$ & $13(1)$ & $-1(1)$ & $-6(1)$ \\
\hline
\end{tabular}




\begin{tabular}{ccccccc}
$\mathrm{C}(21)$ & $11(1)$ & $14(1)$ & $15(1)$ & $1(1)$ & $3(1)$ & $-2(1)$ \\
$\mathrm{C}(3)$ & $18(1)$ & $20(1)$ & $16(1)$ & $3(1)$ & $-4(1)$ & $-5(1)$ \\
\hline
\end{tabular}

\subsection{Crystal Structure Determination of $\mathbf{1 f}$}

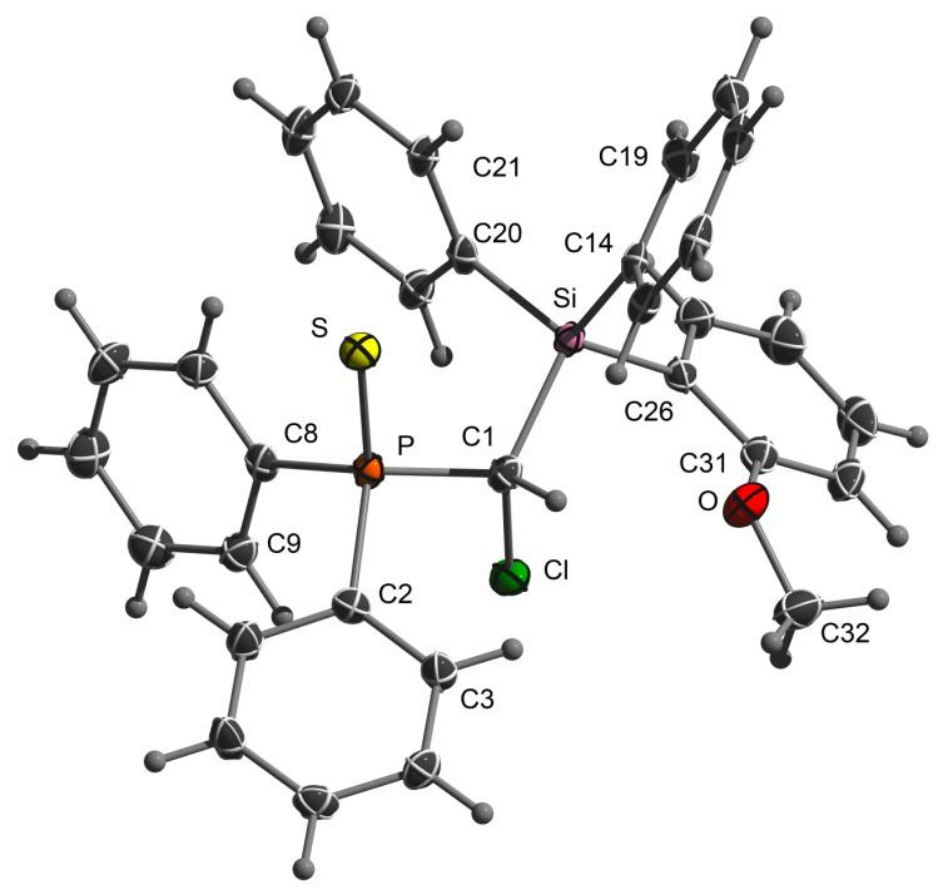

Figure S47. ORTEP Plot of phosphine sulfide 1f. Ellipsoids are drawn at the $50 \%$ probability level

Table S10. Atomic coordinates $\left(\mathrm{x} 10^{4}\right)$ and equivalent isotropic displacement parameters $\left(\AA^{2} \times 10^{3}\right)$ for phosphine sulfide 1f. $\mathrm{U}(\mathrm{eq})$ is defined as one third of the trace of the orthogonalized $\mathrm{U}^{\mathrm{ij}}$ tensor.

\begin{tabular}{lrrrr}
\hline & $\mathrm{x}$ & $\mathrm{y}$ & $\mathrm{z}$ & $\mathrm{U}(\mathrm{eq})$ \\
\hline $\mathrm{Cl}(1)$ & $1387(1)$ & $5391(1)$ & $1173(1)$ & $25(1)$ \\
$\mathrm{C}(1)$ & $1601(2)$ & $6867(2)$ & $2323(2)$ & $18(1)$ \\
$\mathrm{S}(1)$ & $2254(1)$ & $9654(1)$ & $2877(1)$ & $20(1)$ \\
$\mathrm{P}(1)$ & $1899(1)$ & $8073(1)$ & $1628(1)$ & $16(1)$ \\
$\mathrm{Si}(1)$ & $2700(1)$ & $6846(1)$ & $3675(1)$ & $15(1)$ \\
$\mathrm{O}(1)$ & $347(2)$ & $5330(2)$ & $3625(2)$ & $30(1)$ \\
$\mathrm{C}(3)$ & $-644(2)$ & $7446(2)$ & $789(2)$ & $20(1)$ \\
$\mathrm{C}(2)$ & $508(2)$ & $7948(2)$ & $619(2)$ & $18(1)$ \\
$\mathrm{C}(4)$ & $-1699(2)$ & $7481(2)$ & $65(2)$ & $23(1)$ \\
$\mathrm{C}(5)$ & $-1606(2)$ & $8015(2)$ & $-842(2)$ & $23(1)$ \\
$\mathrm{C}(6)$ & $-465(2)$ & $8507(2)$ & $-1030(2)$ & $26(1)$ \\
$\mathrm{C}(7)$ & $585(2)$ & $8473(2)$ & $-311(2)$ & $25(1)$ \\
$\mathrm{C}(8)$ & $3117(2)$ & $7738(2)$ & $625(2)$ & $18(1)$
\end{tabular}




\begin{tabular}{rrrrr}
$\mathrm{C}(9)$ & $2918(2)$ & $6797(2)$ & $-476(2)$ & $22(1)$ \\
$\mathrm{C}(10)$ & $3832(2)$ & $6620(2)$ & $-1262(2)$ & $26(1)$ \\
$\mathrm{C}(11)$ & $4941(2)$ & $7374(2)$ & $-961(2)$ & $30(1)$ \\
$\mathrm{C}(14)$ & $2298(2)$ & $7931(2)$ & $5119(2)$ & $17(1)$ \\
$\mathrm{C}(13)$ & $4231(2)$ & $8501(2)$ & $922(2)$ & $22(1)$ \\
$\mathrm{C}(12)$ & $5146(2)$ & $8316(2)$ & $126(2)$ & $28(1)$ \\
$\mathrm{C}(15)$ & $1260(2)$ & $8572(2)$ & $5211(2)$ & $21(1)$ \\
$\mathrm{C}(16)$ & $1014(2)$ & $9344(2)$ & $6320(2)$ & $26(1)$ \\
$\mathrm{C}(17)$ & $1791(2)$ & $9490(2)$ & $7362(2)$ & $27(1)$ \\
$\mathrm{C}(18)$ & $2802(3)$ & $8854(2)$ & $7301(2)$ & $29(1)$ \\
$\mathrm{C}(19)$ & $3048(2)$ & $8076(2)$ & $6192(2)$ & $23(1)$ \\
$\mathrm{C}(20)$ & $4338(2)$ & $7209(2)$ & $3405(2)$ & $16(1)$ \\
$\mathrm{C}(21)$ & $5054(2)$ & $8310(2)$ & $4078(2)$ & $18(1)$ \\
$\mathrm{C}(22)$ & $6261(2)$ & $8582(2)$ & $3856(2)$ & $22(1)$ \\
$\mathrm{C}(23)$ & $6779(2)$ & $7758(2)$ & $2943(2)$ & $24(1)$ \\
$\mathrm{C}(24)$ & $6093(2)$ & $6671(2)$ & $2257(2)$ & $24(1)$ \\
$\mathrm{C}(25)$ & $4884(2)$ & $6399(2)$ & $2486(2)$ & $21(1)$ \\
$\mathrm{C}(26)$ & $2481(2)$ & $5277(2)$ & $3822(2)$ & $19(1)$ \\
$\mathrm{C}(27)$ & $3482(2)$ & $4682(2)$ & $4057(2)$ & $25(1)$ \\
$\mathrm{C}(28)$ & $3312(3)$ & $3550(2)$ & $4240(2)$ & $32(1)$ \\
$\mathrm{C}(29)$ & $2118(3)$ & $2995(2)$ & $4188(2)$ & $34(1)$ \\
$\mathrm{C}(30)$ & $1103(3)$ & $3549(2)$ & $3956(2)$ & $29(1)$ \\
$\mathrm{C}(31)$ & $1295(2)$ & $4686(2)$ & $3789(2)$ & $22(1)$ \\
$\mathrm{C}(32)$ & $-850(2)$ & $4651(3)$ & $3188(3)$ & $37(1)$ \\
\hline & & & & \\
& & & & \\
\hline
\end{tabular}

Table S11. Anisotropic displacement parameters $\left(\AA^{2} \times 10^{3}\right)$ for phosphine sulfide 1f. The anisotropic displacement factor exponent takes the form: $-2 \pi^{2}\left[\mathrm{~h}^{2} \mathrm{a}^{2} \mathrm{U}^{11}+\ldots+2 \mathrm{~h} \mathrm{k} \mathrm{a} \cdot \mathrm{b} \cdot \mathrm{U}^{12}\right]$.

\begin{tabular}{lcccccc}
\hline & $\mathrm{U}^{11}$ & $\mathrm{U}^{22}$ & $\mathrm{U}^{22}$ & $\mathrm{U}^{23}$ & $\mathrm{U}^{13}$ & $\mathrm{U}^{12}$ \\
\hline $\mathrm{Cl}(1)$ & $32(1)$ & $16(1)$ & $23(1)$ & $6(1)$ & $-2(1)$ & $-1(1)$ \\
$\mathrm{C}(1)$ & $19(1)$ & $15(1)$ & $21(1)$ & $7(1)$ & $2(1)$ & $2(1)$ \\
$\mathrm{S}(1)$ & $23(1)$ & $17(1)$ & $21(1)$ & $4(1)$ & $2(1)$ & $4(1)$ \\
$\mathrm{P}(1)$ & $16(1)$ & $15(1)$ & $19(1)$ & $7(1)$ & $2(1)$ & $3(1)$ \\
$\mathrm{Si}(1)$ & $15(1)$ & $14(1)$ & $17(1)$ & $7(1)$ & $2(1)$ & $2(1)$ \\
$\mathrm{O}(1)$ & $22(1)$ & $30(1)$ & $36(1)$ & $10(1)$ & $3(1)$ & $-3(1)$ \\
$\mathrm{C}(3)$ & $19(1)$ & $25(1)$ & $21(1)$ & $11(1)$ & $4(1)$ & $5(1)$ \\
$\mathrm{C}(2)$ & $17(1)$ & $18(1)$ & $17(1)$ & $5(1)$ & $2(1)$ & $4(1)$ \\
$\mathrm{C}(4)$ & $15(1)$ & $26(1)$ & $27(1)$ & $9(1)$ & $5(1)$ & $3(1)$ \\
$\mathrm{C}(5)$ & $16(1)$ & $32(1)$ & $22(1)$ & $7(1)$ & $-1(1)$ & $6(1)$
\end{tabular}




\begin{tabular}{|c|c|c|c|c|c|c|}
\hline$C(6)$ & $22(1)$ & $39(2)$ & $25(1)$ & $20(1)$ & 2(1) & $6(1)$ \\
\hline$C(7)$ & $17(1)$ & $34(1)$ & $28(1)$ & $17(1)$ & $3(1)$ & 1(1) \\
\hline $\mathrm{C}(8)$ & $16(1)$ & 19(1) & 21(1) & $10(1)$ & 2(1) & $5(1)$ \\
\hline $\mathrm{C}(9)$ & 19(1) & 21(1) & $26(1)$ & $9(1)$ & $0(1)$ & 1(1) \\
\hline$C(10)$ & $28(1)$ & $25(1)$ & $26(1)$ & $5(1)$ & $4(1)$ & $8(1)$ \\
\hline $\mathrm{C}(11)$ & $21(1)$ & $36(2)$ & $35(2)$ & $11(1)$ & 11(1) & $10(1)$ \\
\hline $\mathrm{C}(14)$ & 19(1) & $13(1)$ & $22(1)$ & $8(1)$ & $6(1)$ & $-1(1)$ \\
\hline $\mathrm{C}(13)$ & 21(1) & $23(1)$ & $23(1)$ & $8(1)$ & 2(1) & $3(1)$ \\
\hline $\mathrm{C}(12)$ & $16(1)$ & $32(1)$ & $36(2)$ & $10(1)$ & $6(1)$ & 2(1) \\
\hline $\mathrm{C}(15)$ & $18(1)$ & 20(1) & $29(1)$ & $12(1)$ & $6(1)$ & $-1(1)$ \\
\hline$C(16)$ & $25(1)$ & $18(1)$ & $38(2)$ & $12(1)$ & $18(1)$ & $3(1)$ \\
\hline $\mathrm{C}(17)$ & $37(2)$ & $19(1)$ & $25(1)$ & $6(1)$ & $16(1)$ & $-2(1)$ \\
\hline $\mathrm{C}(18)$ & $40(2)$ & $25(1)$ & $22(1)$ & $10(1)$ & $4(1)$ & $-1(1)$ \\
\hline $\mathrm{C}(19)$ & $27(1)$ & 21(1) & $23(1)$ & $9(1)$ & $5(1)$ & $3(1)$ \\
\hline$C(20)$ & $17(1)$ & $16(1)$ & $19(1)$ & $10(1)$ & $3(1)$ & $5(1)$ \\
\hline $\mathrm{C}(21)$ & $20(1)$ & $16(1)$ & 21(1) & $9(1)$ & $4(1)$ & $3(1)$ \\
\hline $\mathrm{C}(22)$ & $21(1)$ & $19(1)$ & $28(1)$ & $12(1)$ & $2(1)$ & $-2(1)$ \\
\hline $\mathrm{C}(23)$ & $18(1)$ & $25(1)$ & $35(1)$ & $15(1)$ & $9(1)$ & $4(1)$ \\
\hline $\mathrm{C}(24)$ & $27(1)$ & 21(1) & $28(1)$ & $9(1)$ & $12(1)$ & $9(1)$ \\
\hline $\mathrm{C}(25)$ & $23(1)$ & $16(1)$ & $24(1)$ & $7(1)$ & 2(1) & 1(1) \\
\hline$C(26)$ & $25(1)$ & $16(1)$ & $15(1)$ & $6(1)$ & 2(1) & $0(1)$ \\
\hline $\mathrm{C}(27)$ & $27(1)$ & $23(1)$ & $26(1)$ & 11(1) & $-1(1)$ & $1(1)$ \\
\hline $\mathrm{C}(28)$ & $42(2)$ & $23(1)$ & $35(2)$ & $13(1)$ & $-3(1)$ & $6(1)$ \\
\hline $\mathrm{C}(29)$ & $55(2)$ & $17(1)$ & $30(1)$ & 11(1) & $-2(1)$ & $-3(1)$ \\
\hline$C(30)$ & $39(2)$ & $20(1)$ & $26(1)$ & $6(1)$ & $4(1)$ & $-10(1)$ \\
\hline $\mathrm{C}(31)$ & $25(1)$ & $20(1)$ & $20(1)$ & $6(1)$ & $4(1)$ & $1(1)$ \\
\hline $\mathrm{C}(32)$ & $25(1)$ & $43(2)$ & $35(2)$ & $7(1)$ & $-2(1)$ & $-8(1)$ \\
\hline
\end{tabular}




\subsection{Crystal Structure Determination of $\mathbf{2 a}$}

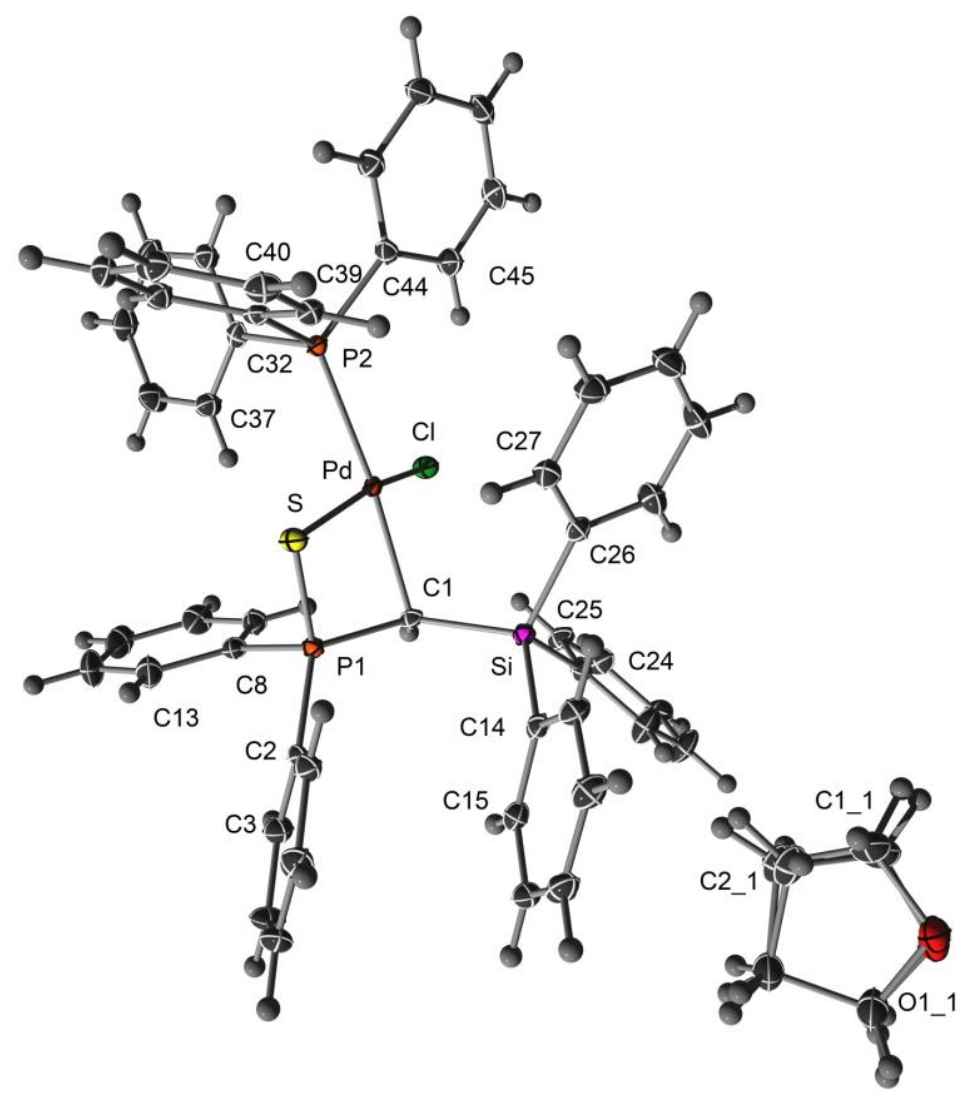

Figure S48. ORTEP Plot of palladium complex 2a. Ellipsoids are drawn at the $50 \%$ probability level

Table S12. Atomic coordinates $\left(\mathrm{x} 10^{4}\right)$ and equivalent isotropic displacement parameters $\left(\AA^{2} \times 10^{3}\right)$ for palladium complex $\mathbf{2 a}$. U(eq) is defined as one third of the trace of the orthogonalized $\mathrm{U}^{\mathrm{ij}}$ tensor.

\begin{tabular}{lllll}
\hline & $\mathrm{X}$ & $\mathrm{y}$ & $\mathrm{Z}$ & $\mathrm{U}(\mathrm{eq})$ \\
\hline $\mathrm{Pd}(1)$ & $2558(1)$ & $7437(1)$ & $934(1)$ & $10(1)$ \\
$\mathrm{C}(1)$ & $3346(2)$ & $6413(1)$ & $1017(1)$ & $11(1)$ \\
$\mathrm{Cl}(1)$ & $4086(1)$ & $7936(1)$ & $1658(1)$ & $15(1)$ \\
$\mathrm{S}(1)$ & $1355(1)$ & $6831(1)$ & $75(1)$ & $14(1)$ \\
$\mathrm{P}(1)$ & $2798(1)$ & $6292(1)$ & $150(1)$ & $11(1)$ \\
$\mathrm{P}(2)$ & $1610(1)$ & $8502(1)$ & $717(1)$ & $11(1)$ \\
$\mathrm{C}(2)$ & $2555(2)$ & $5414(1)$ & $-216(1)$ & $14(1)$ \\
$\mathrm{Si}(1)$ & $2962(1)$ & $5903(1)$ & $1744(1)$ & $11(1)$ \\
$\mathrm{C}(3)$ & $3409(2)$ & $5041(1)$ & $-429(1)$ & $20(1)$ \\
$\mathrm{C}(4)$ & $3207(2)$ & $4364(1)$ & $-694(1)$ & $25(1)$ \\
$\mathrm{C}(5)$ & $2170(2)$ & $4058(1)$ & $-749(1)$ & $23(1)$ \\
$\mathrm{C}(6)$ & $1328(2)$ & $4429(1)$ & $-540(1)$ & $22(1)$ \\
$\mathrm{C}(7)$ & $1515(2)$ & $5108(1)$ & $-275(1)$ & $17(1)$ \\
$\mathrm{C}(8)$ & $3707(2)$ & $6711(1)$ & $-346(1)$ & $16(1)$ \\
$\mathrm{C}(9)$ & $4612(2)$ & $7118(1)$ & $-45(1)$ & $18(1)$ \\
& & & &
\end{tabular}




\begin{tabular}{|c|c|c|c|c|}
\hline$C(10)$ & $5246(2)$ & $7467(1)$ & $-451(1)$ & $24(1)$ \\
\hline$C(11)$ & $4978(2)$ & $7407(1)$ & $-1153(1)$ & $29(1)$ \\
\hline$C(12)$ & $4078(2)$ & $7007(1)$ & $-1457(1)$ & $31(1)$ \\
\hline$C(14)$ & $2312(1)$ & $5020(1)$ & $1478(1)$ & $14(1)$ \\
\hline$C(15)$ & 2922(2) & $4490(1)$ & $1223(1)$ & $17(1)$ \\
\hline$C(16)$ & $2462(2)$ & $3833(1)$ & $1032(1)$ & $18(1)$ \\
\hline$C(17)$ & $1378(2)$ & $3687(1)$ & 1093(1) & $20(1)$ \\
\hline$C(18)$ & $769(2)$ & $4195(1)$ & $1356(1)$ & $21(1)$ \\
\hline $\mathrm{C}(19)$ & $1235(2)$ & $4855(1)$ & $1544(1)$ & $17(1)$ \\
\hline$C(20)$ & $4297(2)$ & $5729(1)$ & $2356(1)$ & $15(1)$ \\
\hline $\mathrm{C}(21)$ & $4461(2)$ & $5102(1)$ & $2729(1)$ & $24(1)$ \\
\hline $\mathrm{C}(22)$ & $5428(2)$ & $4974(1)$ & $3200(1)$ & $27(1)$ \\
\hline $\mathrm{C}(23)$ & $6257(2)$ & $5472(1)$ & $3300(1)$ & $23(1)$ \\
\hline $\mathrm{C}(24)$ & $6120(2)$ & $6100(1)$ & $2936(1)$ & $22(1)$ \\
\hline$C(25)$ & $5146(2)$ & $6230(1)$ & $2470(1)$ & $19(1)$ \\
\hline$C(26)$ & $2018(2)$ & $6404(1)$ & $2208(1)$ & $14(1)$ \\
\hline $\mathrm{C}(27)$ & $963(2)$ & $6638(1)$ & $1885(1)$ & $17(1)$ \\
\hline$C(28)$ & $268(2)$ & $6994(1)$ & $2240(1)$ & $23(1)$ \\
\hline C(29) & $607(2)$ & $7134(1)$ & 2928(1) & $26(1)$ \\
\hline$C(30)$ & $1637(2)$ & $6907(1)$ & $3263(1)$ & $27(1)$ \\
\hline$C(31)$ & $2329(2)$ & $6546(1)$ & $2905(1)$ & $22(1)$ \\
\hline$C(32)$ & 2351(1) & $9103(1)$ & $247(1)$ & $13(1)$ \\
\hline$C(33)$ & $2070(2)$ & $9816(1)$ & $163(1)$ & $17(1)$ \\
\hline $\mathrm{C}(34)$ & $2639(2)$ & $10256(1)$ & $-206(1)$ & $22(1)$ \\
\hline$C(35)$ & $3490(2)$ & $9990(1)$ & $-497(1)$ & $23(1)$ \\
\hline$C(36)$ & $3771(2)$ & $9282(1)$ & $-418(1)$ & $24(1)$ \\
\hline$C(37)$ & $3207(2)$ & $8840(1)$ & $-45(1)$ & $19(1)$ \\
\hline$C(38)$ & $262(1)$ & $8387(1)$ & $163(1)$ & $12(1)$ \\
\hline C(39) & $-518(2)$ & $7969(1)$ & $407(1)$ & $15(1)$ \\
\hline$C(40)$ & $-1548(2)$ & $7842(1)$ & $6(1)$ & $17(1)$ \\
\hline $\mathrm{C}(41)$ & $-1807(2)$ & $8128(1)$ & $-645(1)$ & $17(1)$ \\
\hline$C(42)$ & $-1044(2)$ & $8535(1)$ & $-895(1)$ & $17(1)$ \\
\hline$C(43)$ & $-8(2)$ & $8665(1)$ & $-491(1)$ & $16(1)$ \\
\hline $\mathrm{C}(44)$ & $1290(2)$ & $9051(1)$ & $1408(1)$ & $14(1)$ \\
\hline $\mathrm{C}(45)$ & $2059(2)$ & $9103(1)$ & 2016(1) & $20(1)$ \\
\hline$C(46)$ & $1888(2)$ & $9571(1)$ & $2520(1)$ & $24(1)$ \\
\hline $\mathrm{C}(47)$ & $946(2)$ & $9983(1)$ & $2433(1)$ & $22(1)$ \\
\hline $\mathrm{C}(48)$ & $160(2)$ & $9925(1)$ & 1839(1) & $21(1)$ \\
\hline $\mathrm{C}(49)$ & $332(2)$ & $9465(1)$ & $1328(1)$ & $18(1)$ \\
\hline
\end{tabular}




\begin{tabular}{ccccc} 
C(13) & $3445(2)$ & $6661(1)$ & $-1055(1)$ & $24(1)$ \\
O1A1 & $2203(2)$ & $2622(2)$ & $3562(2)$ & $24(1)$ \\
O1B1 & $2554(13)$ & $2575(11)$ & $3641(11)$ & $24(1)$ \\
C1A1 & $1951(3)$ & $3331(1)$ & $3310(2)$ & $31(1)$ \\
C2A1 & $2806(2)$ & $3506(1)$ & $2885(1)$ & $24(1)$ \\
C1B1 & $2631(16)$ & $3313(8)$ & $3565(9)$ & $31(1)$ \\
C2B1 & $2224(14)$ & $3413(8)$ & $2805(7)$ & $24(1)$ \\
C31 & $2951(2)$ & $2783(1)$ & $2570(1)$ & $29(1)$ \\
C41 & $2935(2)$ & $2288(1)$ & $3165(1)$ & $27(1)$ \\
\hline
\end{tabular}

Table S13. Anisotropic displacement parameters $\left(\AA^{2} \times 10^{3}\right)$ for palladium complex 2a. The anisotropic displacement factor exponent takes the form: $-2 \pi^{2}\left[\mathrm{~h}^{2} \mathrm{a}^{2} \mathrm{U}^{11}+\ldots+2 \mathrm{~h} \mathrm{k} \mathrm{a} \cdot \mathrm{b} \cdot \mathrm{U}^{12}\right]$.

\begin{tabular}{|c|c|c|c|c|c|c|}
\hline & $\mathrm{U}^{11}$ & $\mathrm{U}^{22}$ & $\mathrm{U}^{22}$ & $\mathrm{U}^{23}$ & $\mathrm{U}^{13}$ & $\mathrm{U}^{12}$ \\
\hline $\operatorname{Pd}(1)$ & 11(1) & $8(1)$ & $9(1)$ & $0(1)$ & $0(1)$ & 1(1) \\
\hline $\mathrm{C}(1)$ & $10(1)$ & $10(1)$ & 12(1) & $0(1)$ & $0(1)$ & 1(1) \\
\hline $\mathrm{Cl}(1)$ & $15(1)$ & 13(1) & 15(1) & $-3(1)$ & $-4(1)$ & $0(1)$ \\
\hline$S(1)$ & $13(1)$ & 12(1) & $14(1)$ & $-3(1)$ & $-2(1)$ & 2(1) \\
\hline $\mathrm{P}(1)$ & $13(1)$ & $10(1)$ & $10(1)$ & $-1(1)$ & $0(1)$ & 2(1) \\
\hline $\mathrm{P}(2)$ & $12(1)$ & $9(1)$ & 10(1) & $0(1)$ & $0(1)$ & 1(1) \\
\hline$C(2)$ & $20(1)$ & $10(1)$ & $9(1)$ & $-1(1)$ & $-1(1)$ & $3(1)$ \\
\hline $\operatorname{Si}(1)$ & $12(1)$ & 11(1) & 11(1) & $1(1)$ & $1(1)$ & 1(1) \\
\hline$C(3)$ & $22(1)$ & 18(1) & $20(1)$ & $-1(1)$ & $3(1)$ & $4(1)$ \\
\hline $\mathrm{C}(4)$ & $33(1)$ & 18(1) & $24(1)$ & $-4(1)$ & 7(1) & $10(1)$ \\
\hline $\mathrm{C}(5)$ & $38(1)$ & 13(1) & 18(1) & $-3(1)$ & $1(1)$ & 2(1) \\
\hline $\mathrm{C}(6)$ & $28(1)$ & 17(1) & 18(1) & $-2(1)$ & $-2(1)$ & $-3(1)$ \\
\hline $\mathrm{C}(7)$ & $20(1)$ & 16(1) & 14(1) & $-2(1)$ & $-1(1)$ & $3(1)$ \\
\hline $\mathrm{C}(8)$ & $18(1)$ & 13(1) & $16(1)$ & $1(1)$ & $5(1)$ & 4(1) \\
\hline $\mathrm{C}(9)$ & $18(1)$ & $20(1)$ & 16(1) & 2(1) & $5(1)$ & $3(1)$ \\
\hline $\mathrm{C}(10)$ & $18(1)$ & $28(1)$ & $29(1)$ & $5(1)$ & $8(1)$ & 2(1) \\
\hline $\mathrm{C}(11)$ & $30(1)$ & $31(1)$ & $29(1)$ & $12(1)$ & 17(1) & 7(1) \\
\hline $\mathrm{C}(12)$ & $43(1)$ & $35(1)$ & 15(1) & $5(1)$ & $9(1)$ & 4(1) \\
\hline $\mathrm{C}(14)$ & $16(1)$ & 12(1) & 12(1) & $3(1)$ & $0(1)$ & 1(1) \\
\hline $\mathrm{C}(15)$ & 19(1) & $17(1)$ & 14(1) & $4(1)$ & $2(1)$ & 2(1) \\
\hline$C(16)$ & $25(1)$ & 14(1) & 16(1) & $0(1)$ & $3(1)$ & $5(1)$ \\
\hline $\mathrm{C}(17)$ & $27(1)$ & 13(1) & 19(1) & 1(1) & $-2(1)$ & $-2(1)$ \\
\hline $\mathrm{C}(18)$ & 19(1) & 18(1) & $24(1)$ & $3(1)$ & $2(1)$ & $-3(1)$ \\
\hline $\mathrm{C}(19)$ & $17(1)$ & 14(1) & 19(1) & 2(1) & $2(1)$ & $3(1)$ \\
\hline $\mathrm{C}(20)$ & $16(1)$ & $17(1)$ & 11(1) & $0(1)$ & 1(1) & $2(1)$ \\
\hline
\end{tabular}




\begin{tabular}{|c|c|c|c|c|c|c|}
\hline $\mathrm{C}(21)$ & $22(1)$ & $22(1)$ & $26(1)$ & $10(1)$ & $-2(1)$ & $-4(1)$ \\
\hline $\mathrm{C}(22)$ & 29(1) & $24(1)$ & $26(1)$ & $12(1)$ & $-4(1)$ & 2(1) \\
\hline $\mathrm{C}(23)$ & $20(1)$ & $26(1)$ & 19(1) & $0(1)$ & $-6(1)$ & $8(1)$ \\
\hline $\mathrm{C}(24)$ & 21(1) & 19(1) & 21(1) & $-2(1)$ & $-6(1)$ & $-2(1)$ \\
\hline$C(25)$ & 21(1) & 14(1) & $18(1)$ & 1(1) & $-3(1)$ & $1(1)$ \\
\hline$C(26)$ & 17(1) & 11(1) & $16(1)$ & 1(1) & $5(1)$ & $-2(1)$ \\
\hline $\mathrm{C}(27)$ & 19(1) & $16(1)$ & $16(1)$ & 1(1) & $4(1)$ & $0(1)$ \\
\hline $\mathrm{C}(28)$ & $20(1)$ & 21(1) & $29(1)$ & $3(1)$ & $10(1)$ & $4(1)$ \\
\hline $\mathrm{C}(29)$ & $30(1)$ & $25(1)$ & $29(1)$ & $-3(1)$ & $17(1)$ & $4(1)$ \\
\hline $\mathrm{C}(30)$ & $33(1)$ & $34(1)$ & $18(1)$ & $-7(1)$ & 11(1) & $-2(1)$ \\
\hline $\mathrm{C}(31)$ & 21(1) & $26(1)$ & $17(1)$ & $-1(1)$ & $4(1)$ & $-1(1)$ \\
\hline $\mathrm{C}(32)$ & $15(1)$ & $13(1)$ & $10(1)$ & $-1(1)$ & $-2(1)$ & $-3(1)$ \\
\hline $\mathrm{C}(33)$ & $18(1)$ & $16(1)$ & $15(1)$ & $0(1)$ & $1(1)$ & $1(1)$ \\
\hline $\mathrm{C}(34)$ & $30(1)$ & $15(1)$ & 19(1) & $4(1)$ & $1(1)$ & $-1(1)$ \\
\hline $\mathrm{C}(35)$ & $28(1)$ & $22(1)$ & 21(1) & $5(1)$ & $6(1)$ & $-7(1)$ \\
\hline$C(36)$ & $23(1)$ & $26(1)$ & $25(1)$ & $0(1)$ & $11(1)$ & $-1(1)$ \\
\hline $\mathrm{C}(37)$ & 21(1) & 14(1) & 20(1) & $0(1)$ & $3(1)$ & 2(1) \\
\hline $\mathrm{C}(38)$ & 13(1) & $9(1)$ & $14(1)$ & $-3(1)$ & $0(1)$ & $3(1)$ \\
\hline $\mathrm{C}(39)$ & 18(1) & 13(1) & $14(1)$ & $1(1)$ & 2(1) & $3(1)$ \\
\hline $\mathrm{C}(40)$ & 15(1) & $13(1)$ & $22(1)$ & $-1(1)$ & $4(1)$ & $-1(1)$ \\
\hline $\mathrm{C}(41)$ & 13(1) & $17(1)$ & $18(1)$ & $-3(1)$ & $-2(1)$ & 2(1) \\
\hline$C(42)$ & $20(1)$ & $18(1)$ & $13(1)$ & $1(1)$ & $-1(1)$ & $3(1)$ \\
\hline $\mathrm{C}(43)$ & 17(1) & 13(1) & $16(1)$ & 1(1) & 2(1) & $1(1)$ \\
\hline $\mathrm{C}(44)$ & 18(1) & 11(1) & 12(1) & $-1(1)$ & $4(1)$ & $-1(1)$ \\
\hline $\mathrm{C}(45)$ & $23(1)$ & $21(1)$ & $16(1)$ & $-2(1)$ & $0(1)$ & $4(1)$ \\
\hline $\mathrm{C}(46)$ & $32(1)$ & $26(1)$ & $13(1)$ & $-4(1)$ & $0(1)$ & $1(1)$ \\
\hline $\mathrm{C}(47)$ & $32(1)$ & $18(1)$ & $18(1)$ & $-3(1)$ & $13(1)$ & $-2(1)$ \\
\hline $\mathrm{C}(48)$ & $22(1)$ & 19(1) & $25(1)$ & $-1(1)$ & $10(1)$ & $3(1)$ \\
\hline $\mathrm{C}(49)$ & $18(1)$ & $18(1)$ & $17(1)$ & $0(1)$ & 2(1) & $1(1)$ \\
\hline $\mathrm{C}(13)$ & $33(1)$ & $23(1)$ & $16(1)$ & 1(1) & $3(1)$ & $-1(1)$ \\
\hline O1A1 & $25(1)$ & $22(1)$ & $29(1)$ & 2(1) & $14(1)$ & $0(1)$ \\
\hline O1B1 & $25(1)$ & $22(1)$ & $29(1)$ & 2(1) & $14(1)$ & $0(1)$ \\
\hline $\mathrm{C} 1 \mathrm{~A} 1$ & $43(2)$ & $17(1)$ & $36(2)$ & $-3(1)$ & $16(1)$ & $-1(1)$ \\
\hline $\mathrm{C} 2 \mathrm{~A} 1$ & $25(2)$ & $23(1)$ & $22(1)$ & $3(1)$ & $0(1)$ & $-10(1)$ \\
\hline C1B1 & $43(2)$ & $17(1)$ & $36(2)$ & $-3(1)$ & $16(1)$ & $-1(1)$ \\
\hline $\mathrm{C} 2 \mathrm{~B} 1$ & $25(2)$ & $23(1)$ & $22(1)$ & $3(1)$ & $0(1)$ & $-10(1)$ \\
\hline C31 & $37(1)$ & $29(1)$ & $23(1)$ & $6(1)$ & $12(1)$ & $6(1)$ \\
\hline C41 & $28(1)$ & $30(1)$ & $25(1)$ & $9(1)$ & $11(1)$ & $5(1)$ \\
\hline
\end{tabular}




\subsection{Crystal Structure Determination of $\mathbf{2 b}$}

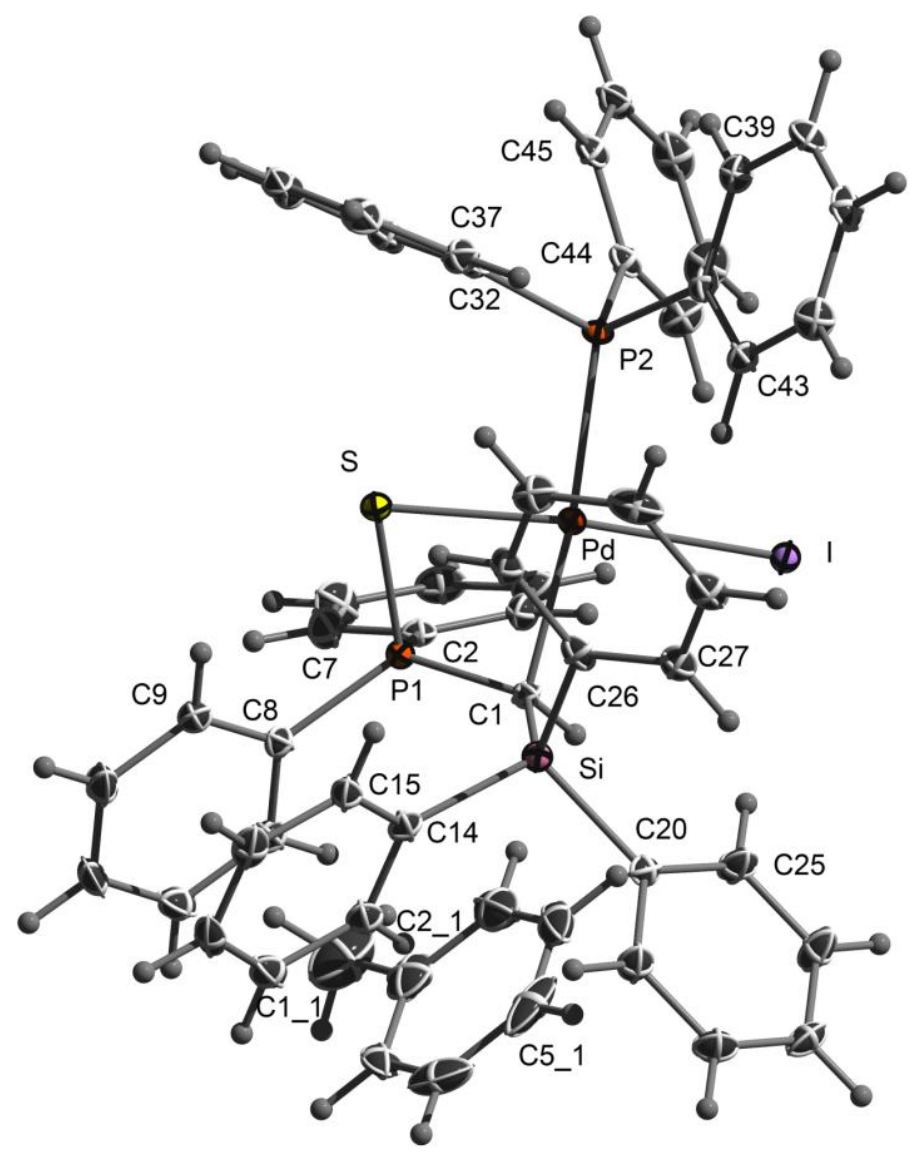

Figure S49. ORTEP Plot of palladium complex $\mathbf{2 b}$. Ellipsoids are drawn at the $50 \%$ probability level

Table S14. Atomic coordinates $\left(\mathrm{x} 10^{4}\right)$ and equivalent isotropic displacement parameters $\left(\AA^{2} \times 10^{3}\right)$ for palladium complex $\mathbf{2 b}$. $U(e q)$ is defined as one third of the trace of the orthogonalized $\mathrm{U}^{\mathrm{ij}}$ tensor.

\begin{tabular}{crrrr}
\hline & $\mathrm{X}$ & $\mathrm{y}$ & $\mathrm{Z}$ & $\mathrm{U}(\mathrm{eq})$ \\
\hline $\mathrm{I}(1)$ & $3525(1)$ & $1943(1)$ & $2600(1)$ & $13(1)$ \\
$\mathrm{Pd}(1)$ & $3861(1)$ & $2669(1)$ & $1255(1)$ & $10(1)$ \\
$\mathrm{S}(1)$ & $3938(1)$ & $3383(1)$ & $80(1)$ & $14(1)$ \\
$\mathrm{P}(1)$ & $3011(1)$ & $3750(1)$ & $884(1)$ & $11(1)$ \\
$\mathrm{C}(1)$ & $3397(2)$ & $3452(1)$ & $1953(2)$ & $11(1)$ \\
$\mathrm{P}(2)$ & $4379(1)$ & $1910(1)$ & $370(1)$ & $10(1)$ \\
$\mathrm{C}(2)$ & $1859(2)$ & $3478(1)$ & $542(2)$ & $12(1)$ \\
$\mathrm{C}(3)$ & $1523(2)$ & $2958(1)$ & $911(2)$ & $16(1)$ \\
$\mathrm{C}(4)$ & $668(2)$ & $2738(1)$ & $588(2)$ & $20(1)$ \\
$\mathrm{C}(5)$ & $146(2)$ & $3031(1)$ & $-98(2)$ & $22(1)$ \\
$\mathrm{Si}(6)$ & $4419(1)$ & $3750(1)$ & $2676(1)$ & $11(1)$ \\
$\mathrm{C}(6)$ & $476(2)$ & $3544(1)$ & $-473(2)$ & $26(1)$ \\
$\mathrm{C}(7)$ & $1332(2)$ & $3767(1)$ & $-159(2)$ & $20(1)$
\end{tabular}




\begin{tabular}{|c|c|c|c|c|}
\hline $\mathrm{C}(8)$ & 2929(2) & $4544(1)$ & $717(2)$ & $14(1)$ \\
\hline $\mathrm{C}(9)$ & $3598(2)$ & $4844(1)$ & $266(2)$ & $17(1)$ \\
\hline$C(10)$ & $3529(2)$ & $5455(1)$ & $144(2)$ & $23(1)$ \\
\hline $\mathrm{C}(11)$ & 2799(2) & $5763(1)$ & $471(2)$ & $24(1)$ \\
\hline$C(12)$ & $2135(2)$ & $5466(1)$ & $933(2)$ & $21(1)$ \\
\hline$C(13)$ & 2198(2) & $4858(1)$ & $1056(2)$ & $17(1)$ \\
\hline $\mathrm{C}(14)$ & $4684(2)$ & $4553(1)$ & $2431(2)$ & $15(1)$ \\
\hline$C(15)$ & $5521(2)$ & $4742(1)$ & $2123(2)$ & $16(1)$ \\
\hline$C(16)$ & $5690(2)$ & $5339(1)$ & $1957(2)$ & $20(1)$ \\
\hline $\mathrm{C}(17)$ & $5024(2)$ & $5759(1)$ & $2103(2)$ & $23(1)$ \\
\hline $\mathrm{C}(18)$ & 4191(2) & $5583(1)$ & $2429(2)$ & $22(1)$ \\
\hline C(19) & $4028(2)$ & 4989(1) & $2593(2)$ & $17(1)$ \\
\hline$C(20)$ & $4123(2)$ & $3731(1)$ & $3913(2)$ & $12(1)$ \\
\hline $\mathrm{C}(21)$ & $4486(2)$ & $4155(1)$ & $4535(2)$ & $18(1)$ \\
\hline$C(22)$ & $4306(2)$ & $4140(1)$ & $5456(2)$ & $22(1)$ \\
\hline $\mathrm{C}(23)$ & $3749(2)$ & $3700(1)$ & $5774(2)$ & $19(1)$ \\
\hline $\mathrm{C}(24)$ & $3375(2)$ & $3279(1)$ & $5174(2)$ & $22(1)$ \\
\hline$C(25)$ & $3562(2)$ & $3293(1)$ & $4252(2)$ & $20(1)$ \\
\hline$C(26)$ & $5511(2)$ & $3314(1)$ & $2560(2)$ & $13(1)$ \\
\hline $\mathrm{C}(27)$ & $5938(2)$ & $3006(1)$ & $3304(2)$ & $15(1)$ \\
\hline $\mathrm{C}(28)$ & $6766(2)$ & $2706(1)$ & $3240(2)$ & $19(1)$ \\
\hline $\mathrm{C}(29)$ & $7192(2)$ & 2711(1) & $2425(2)$ & $20(1)$ \\
\hline$C(30)$ & $6793(2)$ & $3017(1)$ & $1674(2)$ & $19(1)$ \\
\hline $\mathrm{C}(31)$ & $5960(2)$ & $3307(1)$ & $1737(2)$ & $15(1)$ \\
\hline$C(32)$ & $4980(2)$ & $2205(1)$ & $-583(2)$ & $11(1)$ \\
\hline $\mathrm{C}(33)$ & $4503(2)$ & $2376(1)$ & $-1400(2)$ & $15(1)$ \\
\hline $\mathrm{C}(34)$ & $4959(2)$ & $2640(1)$ & $-2094(2)$ & $17(1)$ \\
\hline$C(35)$ & $5897(2)$ & $2751(1)$ & $-1966(2)$ & $18(1)$ \\
\hline$C(36)$ & $6376(2)$ & $2598(1)$ & $-1151(2)$ & $19(1)$ \\
\hline $\mathrm{C}(37)$ & $5926(2)$ & $2320(1)$ & $-462(2)$ & $15(1)$ \\
\hline $\mathrm{C}(38)$ & $5247(2)$ & 1411(1) & $908(2)$ & 11(1) \\
\hline C(39) & $5488(2)$ & $890(1)$ & $469(2)$ & $17(1)$ \\
\hline $\mathrm{C}(40)$ & $6217(2)$ & $546(1)$ & $837(2)$ & $19(1)$ \\
\hline $\mathrm{C}(41)$ & $6707(2)$ & $720(1)$ & $1640(2)$ & $18(1)$ \\
\hline $\mathrm{C}(42)$ & $6470(2)$ & $1234(1)$ & $2077(2)$ & $18(1)$ \\
\hline$C(43)$ & $5741(2)$ & 1581(1) & $1718(2)$ & $14(1)$ \\
\hline $\mathrm{C}(44)$ & $3470(2)$ & $1439(1)$ & $-166(2)$ & $12(1)$ \\
\hline$C(45)$ & $3551(2)$ & $1160(1)$ & $-1013(2)$ & $16(1)$ \\
\hline$C(46)$ & $2835(2)$ & $808(1)$ & $-1392(2)$ & $20(1)$ \\
\hline
\end{tabular}




\begin{tabular}{rrrrr}
$\mathrm{C}(47)$ & $2060(2)$ & $711(1)$ & $-924(2)$ & $22(1)$ \\
$\mathrm{C}(48)$ & $1979(2)$ & $979(1)$ & $-83(2)$ & $24(1)$ \\
$\mathrm{C}(49)$ & $2673(2)$ & $1348(1)$ & $288(2)$ & $19(1)$ \\
$\mathrm{C} 11$ & $-749(2)$ & $4732(2)$ & $1423(2)$ & $55(1)$ \\
$\mathrm{C} 21$ & $40(2)$ & $4621(1)$ & $2138(2)$ & $32(1)$ \\
$\mathrm{C} 31$ & $369(2)$ & $4063(1)$ & $2302(2)$ & $35(1)$ \\
$\mathrm{C} 41$ & $1090(3)$ & $3961(1)$ & $2921(2)$ & $41(1)$ \\
$\mathrm{C} 51$ & $1524(2)$ & $4414(2)$ & $3401(2)$ & $43(1)$ \\
$\mathrm{C} 61$ & $1201(2)$ & $4996(2)$ & $3248(2)$ & $39(1)$ \\
$\mathrm{C} 71$ & $457(2)$ & $5091(1)$ & $2617(2)$ & $30(1)$ \\
\hline
\end{tabular}

Table S15. Anisotropic displacement parameters $\left(\AA^{2} \times 10^{3}\right)$ for palladium complex $2 \mathbf{b}$. The anisotropic displacement factor exponent takes the form: $-2 \pi^{2}\left[\mathrm{~h}^{2} \mathrm{a}^{2} \mathrm{U}^{11}+\ldots+2 \mathrm{~h} \mathrm{k} \mathrm{a} \cdot \mathrm{b} \cdot \mathrm{U}^{12}\right]$.

\begin{tabular}{|c|c|c|c|c|c|c|}
\hline & $\mathrm{U}^{11}$ & $\mathrm{U}^{22}$ & $\mathrm{U}^{22}$ & $\mathrm{U}^{23}$ & $\mathrm{U}^{13}$ & $\mathrm{U}^{12}$ \\
\hline $\mathrm{I}(1)$ & $15(1)$ & 14(1) & 12(1) & 2(1) & 2(1) & 1(1) \\
\hline $\operatorname{Pd}(1)$ & 11(1) & $9(1)$ & $9(1)$ & $0(1)$ & 1(1) & 1(1) \\
\hline$S(1)$ & $18(1)$ & 13(1) & 12(1) & 1(1) & $5(1)$ & $4(1)$ \\
\hline $\mathrm{P}(1)$ & $14(1)$ & 10(1) & 10(1) & $0(1)$ & 1(1) & 2(1) \\
\hline $\mathrm{C}(1)$ & $12(1)$ & 11(1) & 10(1) & $-1(1)$ & $4(1)$ & $-1(1)$ \\
\hline $\mathrm{P}(2)$ & $10(1)$ & 11(1) & 11(1) & $0(1)$ & 1(1) & 1(1) \\
\hline$C(2)$ & 11(1) & 12(1) & 12(1) & $-2(1)$ & 2(1) & 2(1) \\
\hline $\mathrm{C}(3)$ & 19(1) & $16(1)$ & 13(1) & 1(1) & 1(1) & 2(1) \\
\hline$C(4)$ & $19(2)$ & $23(2)$ & 18(1) & $-2(1)$ & $3(1)$ & $-4(1)$ \\
\hline$C(5)$ & $17(2)$ & $27(2)$ & 21(2) & $-7(1)$ & $-3(1)$ & $-3(1)$ \\
\hline $\operatorname{Si}(6)$ & $13(1)$ & 11(1) & 11(1) & $0(1)$ & 1(1) & $0(1)$ \\
\hline$C(6)$ & $25(2)$ & $28(2)$ & $24(2)$ & 1(1) & $-11(1)$ & $5(1)$ \\
\hline$C(7)$ & $24(2)$ & $17(1)$ & 19(1) & 1(1) & $-4(1)$ & $-2(1)$ \\
\hline $\mathrm{C}(8)$ & $18(1)$ & $12(1)$ & 11(1) & $0(1)$ & $-2(1)$ & 2(1) \\
\hline $\mathrm{C}(9)$ & $24(2)$ & 14(1) & 12(1) & $-1(1)$ & 2(1) & 2(1) \\
\hline $\mathrm{C}(10)$ & $35(2)$ & $15(1)$ & 19(2) & $4(1)$ & $3(1)$ & $-6(1)$ \\
\hline $\mathrm{C}(11)$ & $40(2)$ & $10(1)$ & $22(2)$ & $3(1)$ & $-3(1)$ & 2(1) \\
\hline $\mathrm{C}(12)$ & $26(2)$ & 16(1) & 20(2) & $-3(1)$ & $-2(1)$ & $7(1)$ \\
\hline $\mathrm{C}(13)$ & $20(2)$ & $16(1)$ & 14(1) & $0(1)$ & $-1(1)$ & $0(1)$ \\
\hline $\mathrm{C}(14)$ & $19(1)$ & $15(1)$ & 10(1) & $-1(1)$ & $-3(1)$ & $-1(1)$ \\
\hline $\mathrm{C}(15)$ & $22(2)$ & 14(1) & 13(1) & $2(1)$ & $0(1)$ & $-2(1)$ \\
\hline $\mathrm{C}(16)$ & $26(2)$ & $19(1)$ & $14(1)$ & 1(1) & $-4(1)$ & $-9(1)$ \\
\hline $\mathrm{C}(17)$ & $42(2)$ & $12(1)$ & 14(1) & 1(1) & $-7(1)$ & $-8(1)$ \\
\hline$C(18)$ & $34(2)$ & $16(1)$ & $16(1)$ & $-2(1)$ & $-6(1)$ & $5(1)$ \\
\hline
\end{tabular}




\begin{tabular}{|c|c|c|c|c|c|c|}
\hline $\mathrm{C}(19)$ & $23(2)$ & $16(1)$ & $14(1)$ & $-1(1)$ & $-2(1)$ & $-2(1)$ \\
\hline$C(20)$ & $12(1)$ & $14(1)$ & 11(1) & $0(1)$ & $-1(1)$ & $4(1)$ \\
\hline $\mathrm{C}(21)$ & $21(2)$ & $18(1)$ & $15(1)$ & $1(1)$ & $-1(1)$ & $-4(1)$ \\
\hline$C(22)$ & $27(2)$ & $21(2)$ & $18(1)$ & $-6(1)$ & $-1(1)$ & $-3(1)$ \\
\hline$C(23)$ & $19(2)$ & $28(2)$ & $10(1)$ & $-1(1)$ & $1(1)$ & $3(1)$ \\
\hline$C(24)$ & $23(2)$ & $27(2)$ & $17(1)$ & $2(1)$ & $5(1)$ & $-9(1)$ \\
\hline$C(25)$ & $26(2)$ & $20(1)$ & $13(1)$ & $-4(1)$ & $1(1)$ & $-7(1)$ \\
\hline$C(26)$ & $12(1)$ & $10(1)$ & $17(1)$ & $-1(1)$ & $-1(1)$ & $-3(1)$ \\
\hline$C(27)$ & $14(1)$ & $15(1)$ & $16(1)$ & $-1(1)$ & $0(1)$ & $-3(1)$ \\
\hline$C(28)$ & $19(2)$ & $16(1)$ & $21(1)$ & $-2(1)$ & $-7(1)$ & $-1(1)$ \\
\hline$C(29)$ & $9(1)$ & $21(1)$ & $29(2)$ & $-11(1)$ & $-4(1)$ & $0(1)$ \\
\hline$C(30)$ & $15(1)$ & $21(1)$ & $20(1)$ & $-4(1)$ & $4(1)$ & $-4(1)$ \\
\hline $\mathrm{C}(31)$ & $16(1)$ & $14(1)$ & $15(1)$ & $1(1)$ & $0(1)$ & $-3(1)$ \\
\hline$C(32)$ & $14(1)$ & $7(1)$ & $13(1)$ & $-1(1)$ & $5(1)$ & $2(1)$ \\
\hline$C(33)$ & $15(1)$ & $13(1)$ & $17(1)$ & $-1(1)$ & $0(1)$ & $1(1)$ \\
\hline$C(34)$ & $25(2)$ & $14(1)$ & $13(1)$ & $1(1)$ & $2(1)$ & $2(1)$ \\
\hline $\mathrm{C}(35)$ & $25(2)$ & 11(1) & $20(1)$ & $2(1)$ & $12(1)$ & $3(1)$ \\
\hline $\mathrm{C}(36)$ & $14(1)$ & $17(1)$ & $26(2)$ & $0(1)$ & $7(1)$ & $0(1)$ \\
\hline$C(37)$ & $16(1)$ & $16(1)$ & $15(1)$ & $-2(1)$ & 2(1) & $-1(1)$ \\
\hline$C(38)$ & $8(1)$ & $13(1)$ & $13(1)$ & $3(1)$ & $3(1)$ & $1(1)$ \\
\hline C(39) & $20(2)$ & $16(1)$ & $14(1)$ & $2(1)$ & $1(1)$ & 1(1) \\
\hline $\mathrm{C}(40)$ & $23(2)$ & $14(1)$ & $21(2)$ & $1(1)$ & $4(1)$ & $7(1)$ \\
\hline $\mathrm{C}(41)$ & $14(1)$ & $18(1)$ & $24(2)$ & $6(1)$ & $1(1)$ & $5(1)$ \\
\hline $\mathrm{C}(42)$ & $15(1)$ & $21(1)$ & 19(1) & $0(1)$ & $-2(1)$ & $-2(1)$ \\
\hline $\mathrm{C}(43)$ & $13(1)$ & $14(1)$ & $16(1)$ & $1(1)$ & $3(1)$ & $0(1)$ \\
\hline $\mathrm{C}(44)$ & $12(1)$ & 11(1) & $14(1)$ & $1(1)$ & $-2(1)$ & $1(1)$ \\
\hline $\mathrm{C}(45)$ & $17(1)$ & $13(1)$ & $17(1)$ & $2(1)$ & $2(1)$ & $5(1)$ \\
\hline $\mathrm{C}(46)$ & $27(2)$ & $13(1)$ & $18(1)$ & $-2(1)$ & $-5(1)$ & $2(1)$ \\
\hline $\mathrm{C}(47)$ & $20(2)$ & $18(1)$ & $26(2)$ & $1(1)$ & $-10(1)$ & $-7(1)$ \\
\hline $\mathrm{C}(48)$ & $15(2)$ & $32(2)$ & $24(2)$ & $2(1)$ & $0(1)$ & $-5(1)$ \\
\hline $\mathrm{C}(49)$ & $15(1)$ & $26(2)$ & $16(1)$ & $-2(1)$ & $1(1)$ & $-1(1)$ \\
\hline C11 & $44(2)$ & $80(3)$ & $42(2)$ & $-2(2)$ & $5(2)$ & $5(2)$ \\
\hline $\mathrm{C} 21$ & $29(2)$ & $42(2)$ & $27(2)$ & $5(1)$ & $14(1)$ & $4(1)$ \\
\hline C 31 & $43(2)$ & $27(2)$ & $39(2)$ & $-2(2)$ & $23(2)$ & $-5(2)$ \\
\hline C41 & $60(2)$ & $27(2)$ & $39(2)$ & $13(2)$ & $34(2)$ & $14(2)$ \\
\hline C51 & $29(2)$ & $80(3)$ & $21(2)$ & $16(2)$ & $13(1)$ & $22(2)$ \\
\hline C61 & $41(2)$ & $50(2)$ & $29(2)$ & $-19(2)$ & $16(2)$ & $-8(2)$ \\
\hline C71 & $40(2)$ & $19(2)$ & $34(2)$ & $4(1)$ & $21(2)$ & $14(1)$ \\
\hline
\end{tabular}




\subsection{Crystal Structure Determination of 2c}

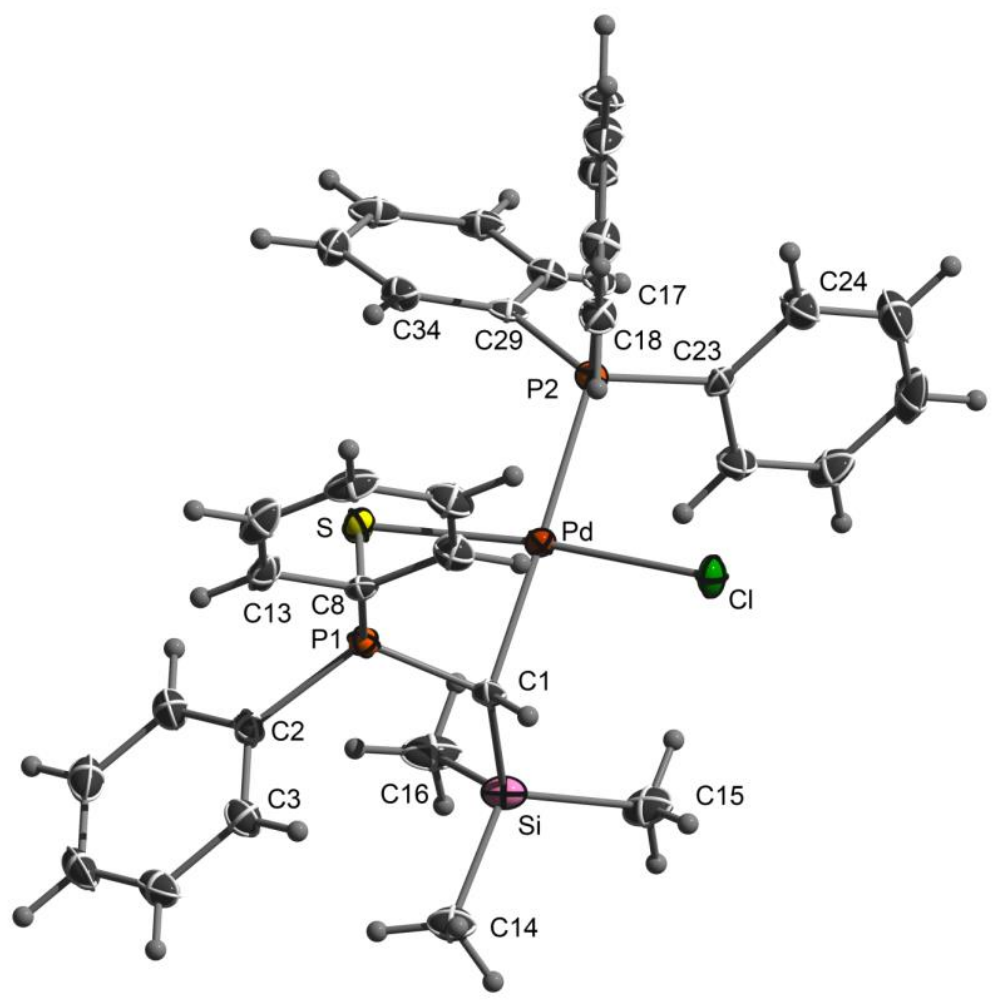

Figure S50. ORTEP Plot of palladium complex 2c. Ellipsoids are drawn at the $50 \%$ probability level

Table S16. Atomic coordinates $\left(\mathrm{x} 10^{4}\right)$ and equivalent isotropic displacement parameters $\left(\AA^{2} \times 10^{3}\right)$ for palladium complex 2c. $\mathrm{U}(\mathrm{eq})$ is defined as one third of the trace of the orthogonalized $\mathrm{U}^{\mathrm{ij}}$ tensor.

\begin{tabular}{lllll}
\hline & $\mathrm{X}$ & $\mathrm{y}$ & $\mathrm{Z}$ & $\mathrm{U}(\mathrm{eq})$ \\
\hline $\mathrm{Pd}(1)$ & $5375(1)$ & $7313(1)$ & $7072(1)$ & $12(1)$ \\
$\mathrm{C}(1)$ & $7331(3)$ & $6420(3)$ & $5952(3)$ & $14(1)$ \\
$\mathrm{Cl}(1)$ & $6184(1)$ & $7450(1)$ & $8329(1)$ & $21(1)$ \\
$\mathrm{S}(1)$ & $4911(1)$ & $6778(1)$ & $5745(1)$ & $19(1)$ \\
$\mathrm{P}(1)$ & $6797(1)$ & $5563(1)$ & $5443(1)$ & $15(1)$ \\
$\mathrm{Si}(1)$ & $7929(1)$ & $7590(1)$ & $5086(1)$ & $19(1)$ \\
$\mathrm{C}(14)$ & $9635(4)$ & $6751(4)$ & $4064(3)$ & $25(1)$ \\
$\mathrm{P}(2)$ & $3208(1)$ & $8306(1)$ & $8253(1)$ & $13(1)$ \\
$\mathrm{C}(2)$ & $7654(4)$ & $5083(3)$ & $4036(3)$ & $18(1)$ \\
$\mathrm{C}(15)$ & $8022(4)$ & $8602(4)$ & $5945(3)$ & $29(1)$ \\
$\mathrm{C}(16)$ & $6812(4)$ & $8575(4)$ & $4382(3)$ & $31(1)$ \\
$\mathrm{C}(3)$ & $8998(4)$ & $4321(3)$ & $3665(3)$ & $22(1)$ \\
$\mathrm{C}(7)$ & $7015(4)$ & $5516(4)$ & $3327(3)$ & $24(1)$ \\
$\mathrm{C}(6)$ & $7712(4)$ & $5182(4)$ & $2243(3)$ & $29(1)$ \\
$\mathrm{C}(5)$ & $9045(4)$ & $4429(4)$ & $1874(3)$ & $29(1)$ \\
$\mathrm{C}(4)$ & $9693(4)$ & $3997(4)$ & $2580(3)$ & $26(1)$ \\
$\mathrm{C}(8)$ & $6843(3)$ & $4174(3)$ & $6202(3)$ & $14(1)$ \\
\hline
\end{tabular}




\begin{tabular}{rrrrr}
$\mathrm{C}(9)$ & $6741(4)$ & $4108(4)$ & $7266(3)$ & $21(1)$ \\
$\mathrm{C}(10)$ & $6628(4)$ & $3099(4)$ & $7874(3)$ & $25(1)$ \\
$\mathrm{C}(11)$ & $6624(4)$ & $2139(4)$ & $7414(3)$ & $25(1)$ \\
$\mathrm{C}(12)$ & $6736(4)$ & $2191(4)$ & $6361(4)$ & $26(1)$ \\
$\mathrm{C}(13)$ & $6848(4)$ & $3200(4)$ & $5750(3)$ & $21(1)$ \\
$\mathrm{C}(17)$ & $2623(4)$ & $7273(3)$ & $9264(3)$ & $15(1)$ \\
$\mathrm{C}(18)$ & $3552(4)$ & $6154(3)$ & $9389(3)$ & $20(1)$ \\
$\mathrm{C}(19)$ & $3135(4)$ & $5327(4)$ & $10131(3)$ & $23(1)$ \\
$\mathrm{C}(20)$ & $1814(4)$ & $5622(4)$ & $10738(3)$ & $25(1)$ \\
$\mathrm{C}(21)$ & $886(4)$ & $6735(4)$ & $10600(3)$ & $24(1)$ \\
$\mathrm{C}(22)$ & $1289(4)$ & $7552(4)$ & $9857(3)$ & $23(1)$ \\
$\mathrm{C}(23)$ & $3033(4)$ & $9582(3)$ & $8954(3)$ & $16(1)$ \\
$\mathrm{C}(24)$ & $2339(4)$ & $9814(4)$ & $10048(3)$ & $25(1)$ \\
$\mathrm{C}(25)$ & $2225(5)$ & $10823(4)$ & $10523(4)$ & $35(1)$ \\
$\mathrm{C}(26)$ & $2782(5)$ & $11621(4)$ & $9903(4)$ & $34(1)$ \\
$\mathrm{C}(27)$ & $3465(4)$ & $11400(4)$ & $8813(4)$ & $28(1)$ \\
$\mathrm{C}(28)$ & $3605(4)$ & $10373(4)$ & $8341(3)$ & $22(1)$ \\
$\mathrm{C}(29)$ & $1903(4)$ & $8999(3)$ & $7713(3)$ & $15(1)$ \\
$\mathrm{C}(30)$ & $1033(4)$ & $10230(3)$ & $7898(3)$ & $18(1)$ \\
$\mathrm{C}(31)$ & $64(4)$ & $10720(4)$ & $7470(3)$ & $21(1)$ \\
$\mathrm{C}(32)$ & $-43(4)$ & $10000(4)$ & $6836(3)$ & $23(1)$ \\
$\mathrm{C}(33)$ & $804(4)$ & $8772(4)$ & $6650(3)$ & $22(1)$ \\
$\mathrm{C}(34)$ & $1754(4)$ & $8264(3)$ & $7103(3)$ & $19(1)$ \\
\hline & & & &
\end{tabular}

Table S17. Anisotropic displacement parameters $\left(\AA^{2} \times 10^{3}\right)$ for palladium complex 2c. The anisotropic displacement factor exponent takes the form: $-2 \pi^{2}\left[\mathrm{~h}^{2} \mathrm{a}^{2} \mathrm{U}^{11}+\ldots+2 \mathrm{~h} \mathrm{k} \mathrm{a} \cdot \mathrm{b} \cdot \mathrm{U}^{12}\right]$.

\begin{tabular}{ccccccc}
\hline & $\mathrm{U}^{11}$ & $\mathrm{U}^{22}$ & $\mathrm{U}^{22}$ & $\mathrm{U}^{23}$ & $\mathrm{U}^{13}$ & $\mathrm{U}^{12}$ \\
\hline $\mathrm{Pd}(1)$ & $12(1)$ & $12(1)$ & $13(1)$ & $0(1)$ & $-5(1)$ & $-4(1)$ \\
$\mathrm{C}(1)$ & $10(2)$ & $14(2)$ & $15(2)$ & $-2(2)$ & $-4(2)$ & $-1(2)$ \\
$\mathrm{Cl}(1)$ & $24(1)$ & $24(1)$ & $20(1)$ & $1(1)$ & $-14(1)$ & $-9(1)$ \\
$\mathrm{S}(1)$ & $15(1)$ & $22(1)$ & $19(1)$ & $-3(1)$ & $-9(1)$ & $-3(1)$ \\
$\mathrm{P}(1)$ & $13(1)$ & $16(1)$ & $14(1)$ & $-1(1)$ & $-4(1)$ & $-4(1)$ \\
$\mathrm{Si}(1)$ & $15(1)$ & $15(1)$ & $22(1)$ & $5(1)$ & $-5(1)$ & $-5(1)$ \\
$\mathrm{C}(14)$ & $23(2)$ & $23(2)$ & $26(2)$ & $5(2)$ & $-6(2)$ & $-11(2)$ \\
$\mathrm{P}(2)$ & $13(1)$ & $12(1)$ & $13(1)$ & $1(1)$ & $-5(1)$ & $-5(1)$ \\
$\mathrm{C}(2)$ & $22(2)$ & $16(2)$ & $16(2)$ & $-3(2)$ & $-5(2)$ & $-8(2)$ \\
$\mathrm{C}(15)$ & $29(3)$ & $20(2)$ & $39(3)$ & $2(2)$ & $-8(2)$ & $-15(2)$ \\
$\mathrm{C}(16)$ & $22(2)$ & $30(3)$ & $33(3)$ & $16(2)$ & $-9(2)$ & $-10(2)$ \\
$\mathrm{C}(3)$ & $23(2)$ & $20(2)$ & $21(2)$ & $-1(2)$ & $-7(2)$ & $-7(2)$
\end{tabular}




\begin{tabular}{|c|c|c|c|c|c|c|}
\hline $\mathrm{C}(7)$ & $25(2)$ & $28(3)$ & $20(2)$ & $-3(2)$ & $-8(2)$ & $-10(2)$ \\
\hline $\mathrm{C}(6)$ & 41(3) & $31(3)$ & $25(2)$ & 1(2) & $-15(2)$ & $-21(2)$ \\
\hline $\mathrm{C}(5)$ & 41(3) & 27(3) & $17(2)$ & $-6(2)$ & $-1(2)$ & $-19(2)$ \\
\hline $\mathrm{C}(4)$ & $26(2)$ & $20(2)$ & $25(2)$ & $-5(2)$ & $1(2)$ & $-12(2)$ \\
\hline $\mathrm{C}(8)$ & $8(2)$ & $18(2)$ & $14(2)$ & $-1(2)$ & $-3(2)$ & $-5(2)$ \\
\hline $\mathrm{C}(9)$ & $20(2)$ & $19(2)$ & $21(2)$ & $1(2)$ & $-7(2)$ & $-6(2)$ \\
\hline$C(10)$ & $23(2)$ & $24(2)$ & 21(2) & $4(2)$ & $-5(2)$ & $-7(2)$ \\
\hline $\mathrm{C}(11)$ & $19(2)$ & $16(2)$ & $38(3)$ & $9(2)$ & $-9(2)$ & $-9(2)$ \\
\hline$C(12)$ & $25(2)$ & $15(2)$ & $42(3)$ & $-2(2)$ & $-16(2)$ & $-7(2)$ \\
\hline$C(13)$ & $16(2)$ & $24(2)$ & $27(2)$ & $-2(2)$ & $-13(2)$ & $-7(2)$ \\
\hline$C(17)$ & $20(2)$ & $16(2)$ & 11(2) & $3(2)$ & $-5(2)$ & $-9(2)$ \\
\hline$C(18)$ & $23(2)$ & $20(2)$ & 19(2) & $6(2)$ & $-10(2)$ & $-11(2)$ \\
\hline $\mathrm{C}(19)$ & $34(3)$ & $17(2)$ & $25(2)$ & $10(2)$ & $-18(2)$ & $-13(2)$ \\
\hline$C(20)$ & $36(3)$ & 29(3) & $18(2)$ & $9(2)$ & $-9(2)$ & $-24(2)$ \\
\hline $\mathrm{C}(21)$ & $19(2)$ & $30(3)$ & $19(2)$ & $-1(2)$ & $4(2)$ & $-15(2)$ \\
\hline$C(22)$ & $23(2)$ & 21(2) & $23(2)$ & 1(2) & $-7(2)$ & $-10(2)$ \\
\hline$C(23)$ & $12(2)$ & $20(2)$ & $18(2)$ & $-1(2)$ & $-8(2)$ & $-6(2)$ \\
\hline$C(24)$ & $29(2)$ & $19(2)$ & $24(2)$ & $-2(2)$ & $-4(2)$ & $-11(2)$ \\
\hline$C(25)$ & $50(3)$ & $36(3)$ & $20(2)$ & $-4(2)$ & $-9(2)$ & $-19(2)$ \\
\hline$C(26)$ & $43(3)$ & $25(3)$ & $42(3)$ & $-10(2)$ & $-19(3)$ & $-15(2)$ \\
\hline$C(27)$ & 28(3) & $22(2)$ & $38(3)$ & 2(2) & $-12(2)$ & $-16(2)$ \\
\hline$C(28)$ & $23(2)$ & $24(2)$ & $19(2)$ & 1(2) & $-5(2)$ & $-12(2)$ \\
\hline$C(29)$ & $13(2)$ & $17(2)$ & 12(2) & $4(2)$ & $0(2)$ & $-8(2)$ \\
\hline$C(30)$ & $15(2)$ & $17(2)$ & $16(2)$ & 1(2) & $-3(2)$ & $-5(2)$ \\
\hline $\mathrm{C}(31)$ & $14(2)$ & $15(2)$ & $25(2)$ & $1(2)$ & $-2(2)$ & $-4(2)$ \\
\hline$C(32)$ & $12(2)$ & $23(2)$ & $24(2)$ & $7(2)$ & $-5(2)$ & $-2(2)$ \\
\hline$C(33)$ & 22(2) & $29(2)$ & 21(2) & $0(2)$ & $-10(2)$ & $-13(2)$ \\
\hline $\mathrm{C}(34)$ & $16(2)$ & $14(2)$ & $22(2)$ & $-2(2)$ & $-5(2)$ & $-5(2)$ \\
\hline
\end{tabular}




\subsection{Crystal Structure Determination of $\mathbf{2 d}$}

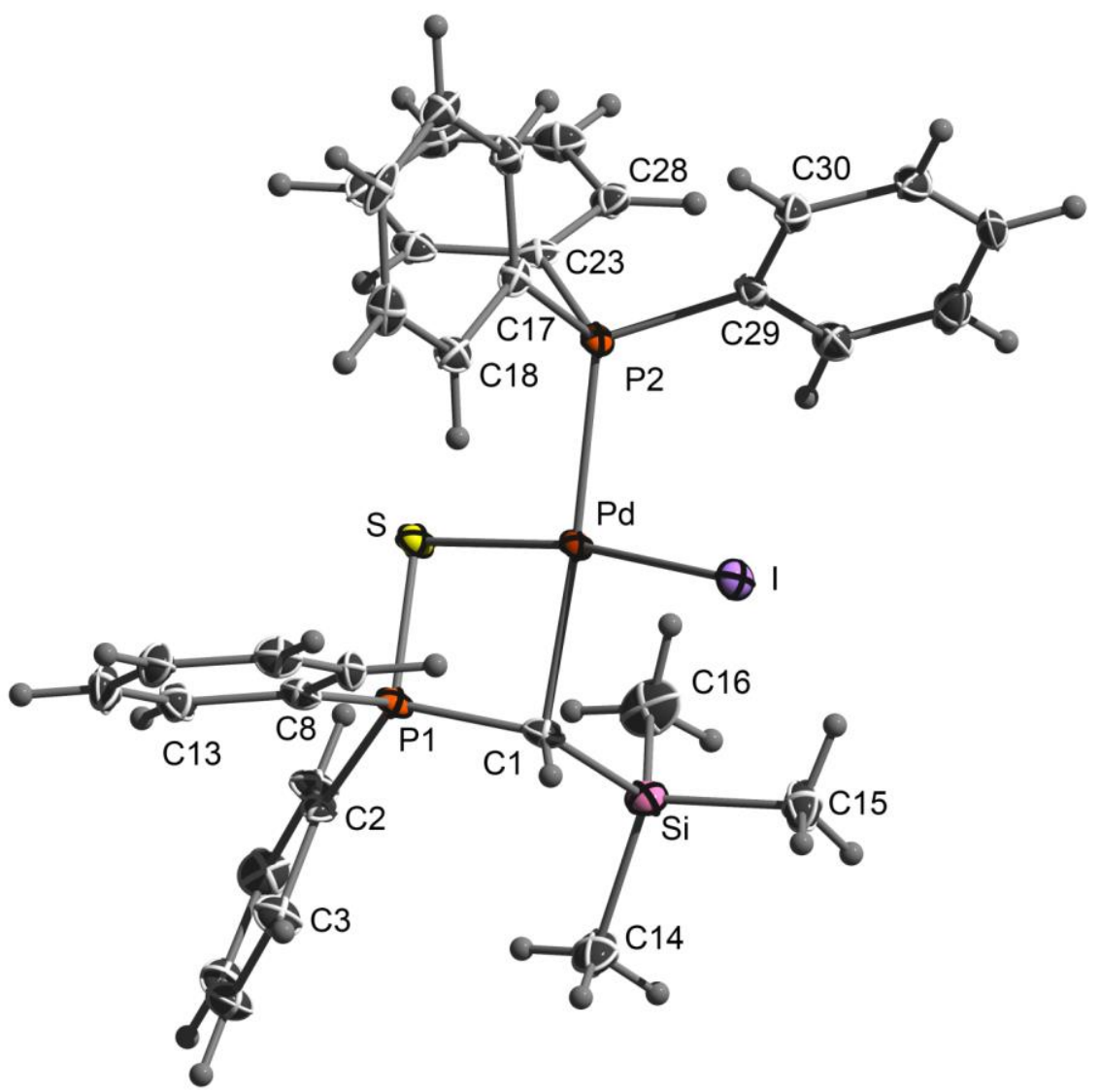

Figure S51. ORTEP Plot of palladium complex 2d. Ellipsoids are drawn at the 50\% probability level

Table S18. Atomic coordinates $\left(\mathrm{x} 10^{4}\right)$ and equivalent isotropic displacement parameters $\left(\AA^{2} \times 10^{3}\right)$ for palladium complex 2d. U(eq) is defined as one third of the trace of the orthogonalized $U^{i j}$ tensor.

\begin{tabular}{crrrr}
\hline & $\mathrm{X}$ & $\mathrm{y}$ & $\mathrm{Z}$ & $\mathrm{U}(\mathrm{eq})$ \\
\hline $\mathrm{Pd}(1)$ & $7772(1)$ & $2551(1)$ & $2791(1)$ & $11(1)$ \\
$\mathrm{S}(1)$ & $9077(1)$ & $3625(1)$ & $3034(1)$ & $15(1)$ \\
$\mathrm{P}(1)$ & $10039(1)$ & $2131(1)$ & $3557(1)$ & $13(1)$ \\
$\mathrm{C}(1)$ & $8607(3)$ & $1683(3)$ & $3942(2)$ & $13(1)$ \\
$\mathrm{I}(2)$ & $6678(1)$ & $1059(1)$ & $2569(1)$ & $16(1)$ \\
$\mathrm{C}(2)$ & $10835(3)$ & $2337(3)$ & $4500(2)$ & $13(1)$ \\
$\mathrm{P}(2)$ & $6990(1)$ & $3544(1)$ & $1530(1)$ & $12(1)$ \\
$\mathrm{C}(16)$ & $6517(4)$ & $3841(3)$ & $5376(3)$ & $34(1)$ \\
$\mathrm{C}(15)$ & $5790(4)$ & $1763(3)$ & $5361(3)$ & $26(1)$ \\
$\mathrm{C}(14)$ & $8166(4)$ & $1717(3)$ & $6197(3)$ & $29(1)$ \\
$\mathrm{C}(3)$ & $11761(4)$ & $1394(3)$ & $4815(3)$ & $20(1)$ \\
$\mathrm{C}(13)$ & $12832(3)$ & $1162(3)$ & $2360(2)$ & $18(1)$ \\
$\mathrm{C}(4)$ & $12291(4)$ & $1528(3)$ & $5586(3)$ & $23(1)$ \\
$\mathrm{Si}(6)$ & $7284(1)$ & $2254(1)$ & $5201(1)$ & $18(1)$ \\
& & & &
\end{tabular}




\begin{tabular}{|c|c|c|c|c|}
\hline$C(6)$ & 10981(4) & $3530(3)$ & $5755(3)$ & $24(1)$ \\
\hline $\mathrm{C}(7)$ & $10439(4)$ & $3403(3)$ & $4979(2)$ & $19(1)$ \\
\hline$C(5)$ & 11909(4) & $2598(3)$ & $6049(2)$ & $22(1)$ \\
\hline $\mathrm{C}(8)$ & $11480(3)$ & 1133(3) & $2579(2)$ & $14(1)$ \\
\hline $\mathrm{C}(9)$ & $11230(3)$ & $378(3)$ & $2005(2)$ & $15(1)$ \\
\hline$C(10)$ & $12320(3)$ & $-331(3)$ & $1222(2)$ & $18(1)$ \\
\hline$C(12)$ & $13910(4)$ & $446(3)$ & $1575(3)$ & $22(1)$ \\
\hline $\mathrm{C}(11)$ & $13656(4)$ & $-294(3)$ & $1003(3)$ & $21(1)$ \\
\hline $\mathrm{C}(17)$ & $8131(3)$ & $2884(3)$ & 292(2) & $12(1)$ \\
\hline $\mathrm{C}(18)$ & $9281(3)$ & $1866(3)$ & $207(2)$ & $15(1)$ \\
\hline $\mathrm{C}(19)$ & 10202(3) & $1355(3)$ & $-723(2)$ & $18(1)$ \\
\hline $\mathrm{C}(20)$ & $9975(3)$ & $1855(3)$ & $-1571(3)$ & $18(1)$ \\
\hline $\mathrm{C}(21)$ & $8842(4)$ & $2879(3)$ & $-1500(2)$ & $19(1)$ \\
\hline $\mathrm{C}(22)$ & $7926(3)$ & $3394(3)$ & $-571(2)$ & $15(1)$ \\
\hline$C(23)$ & $6977(3)$ & $5001(3)$ & $1492(2)$ & $13(1)$ \\
\hline$C(24)$ & $8273(3)$ & $5128(3)$ & 1193(2) & $17(1)$ \\
\hline$C(25)$ & $8335(4)$ & $6200(3)$ & $1203(2)$ & $18(1)$ \\
\hline$C(26)$ & 7093(4) & $7163(3)$ & $1519(2)$ & $21(1)$ \\
\hline $\mathrm{C}(27)$ & $5814(4)$ & $7042(3)$ & $1805(3)$ & $22(1)$ \\
\hline$C(28)$ & $5743(3)$ & $5966(3)$ & $1797(2)$ & $17(1)$ \\
\hline $\mathrm{C}(29)$ & $5195(3)$ & $3710(3)$ & $1576(2)$ & $13(1)$ \\
\hline $\mathrm{C}(30)$ & 4799(4) & $3452(3)$ & $765(3)$ & $19(1)$ \\
\hline $\mathrm{C}(31)$ & $3410(4)$ & $3584(3)$ & $881(3)$ & $24(1)$ \\
\hline $\mathrm{C}(32)$ & $2405(4)$ & $3982(3)$ & $1785(3)$ & $28(1)$ \\
\hline $\mathrm{C}(33)$ & $2792(4)$ & $4229(3)$ & 2601(3) & $25(1)$ \\
\hline $\mathrm{C}(34)$ & $4181(3)$ & $4078(3)$ & $2498(3)$ & 19(1) \\
\hline
\end{tabular}

Table S19. Anisotropic displacement parameters $\left(\AA^{2} \times 10^{3}\right)$ for palladium complex 2d. The anisotropic displacement factor exponent takes the form: $-2 \pi^{2}\left[\mathrm{~h}^{2} \mathrm{a} \cdot{ }^{2} \mathrm{U}^{11}+\ldots+2 \mathrm{~h} \mathrm{k} \mathrm{a} \cdot \mathrm{b} \cdot \mathrm{U}^{12}\right]$.

\begin{tabular}{ccccccc}
\hline & $\mathrm{U}^{11}$ & $\mathrm{U}^{22}$ & $\mathrm{U}^{22}$ & $\mathrm{U}^{23}$ & $\mathrm{U}^{13}$ & $\mathrm{U}^{12}$ \\
\hline $\mathrm{Pd}(1)$ & $12(1)$ & $13(1)$ & $10(1)$ & $0(1)$ & $-5(1)$ & $-5(1)$ \\
$\mathrm{S}(1)$ & $18(1)$ & $15(1)$ & $18(1)$ & $3(1)$ & $-10(1)$ & $-8(1)$ \\
$\mathrm{P}(1)$ & $15(1)$ & $13(1)$ & $14(1)$ & $1(1)$ & $-7(1)$ & $-5(1)$ \\
$\mathrm{C}(1)$ & $16(2)$ & $12(2)$ & $13(2)$ & $2(1)$ & $-8(1)$ & $-3(1)$ \\
$\mathrm{I}(2)$ & $19(1)$ & $15(1)$ & $18(1)$ & $1(1)$ & $-8(1)$ & $-8(1)$ \\
$\mathrm{C}(2)$ & $17(2)$ & $17(2)$ & $9(2)$ & $2(1)$ & $-6(1)$ & $-9(1)$ \\
$\mathrm{P}(2)$ & $12(1)$ & $14(1)$ & $10(1)$ & $-1(1)$ & $-4(1)$ & $-5(1)$ \\
$\mathrm{C}(16)$ & $36(2)$ & $25(2)$ & $24(2)$ & $-7(2)$ & $4(2)$ & $-2(2)$
\end{tabular}




\begin{tabular}{|c|c|c|c|c|c|c|}
\hline $\mathrm{C}(15)$ & $20(2)$ & $39(2)$ & $18(2)$ & $-2(2)$ & $-2(2)$ & $-13(2)$ \\
\hline $\mathrm{C}(14)$ & $31(2)$ & $43(3)$ & $16(2)$ & $4(2)$ & $-6(2)$ & $-20(2)$ \\
\hline$C(3)$ & $25(2)$ & $18(2)$ & $20(2)$ & $-1(2)$ & $-11(2)$ & $-7(2)$ \\
\hline $\mathrm{C}(13)$ & $18(2)$ & $20(2)$ & $20(2)$ & 2(2) & $-9(2)$ & $-8(2)$ \\
\hline $\mathrm{C}(4)$ & $23(2)$ & $23(2)$ & $24(2)$ & $6(2)$ & $-13(2)$ & $-6(2)$ \\
\hline $\mathrm{Si}(6)$ & $19(1)$ & $21(1)$ & $13(1)$ & $0(1)$ & $-4(1)$ & $-7(1)$ \\
\hline$C(6)$ & $33(2)$ & $21(2)$ & $18(2)$ & $-4(2)$ & $-7(2)$ & $-12(2)$ \\
\hline $\mathrm{C}(7)$ & $24(2)$ & $16(2)$ & 19(2) & 1(1) & $-12(2)$ & $-7(2)$ \\
\hline$C(5)$ & $26(2)$ & $35(2)$ & $12(2)$ & 1(2) & $-9(2)$ & $-18(2)$ \\
\hline $\mathrm{C}(8)$ & $15(2)$ & $13(2)$ & $14(2)$ & 2(1) & $-6(1)$ & $-2(1)$ \\
\hline $\mathrm{C}(9)$ & $13(2)$ & $17(2)$ & $18(2)$ & $3(1)$ & $-5(1)$ & $-8(1)$ \\
\hline $\mathrm{C}(10)$ & $23(2)$ & $15(2)$ & $15(2)$ & $-2(1)$ & $-7(2)$ & $-7(2)$ \\
\hline $\mathrm{C}(12)$ & $18(2)$ & $31(2)$ & $23(2)$ & 1(2) & $-6(2)$ & $-16(2)$ \\
\hline $\mathrm{C}(11)$ & $18(2)$ & $23(2)$ & $17(2)$ & $1(2)$ & $-1(2)$ & $-6(2)$ \\
\hline $\mathrm{C}(17)$ & $14(2)$ & $15(2)$ & $9(2)$ & $-2(1)$ & $-2(1)$ & $-9(1)$ \\
\hline $\mathrm{C}(18)$ & $18(2)$ & $17(2)$ & $14(2)$ & $0(1)$ & $-6(1)$ & $-9(2)$ \\
\hline $\mathrm{C}(19)$ & $16(2)$ & $10(2)$ & $24(2)$ & $-6(1)$ & $0(2)$ & $-4(1)$ \\
\hline$C(20)$ & $22(2)$ & $20(2)$ & $15(2)$ & $-4(1)$ & $3(2)$ & $-16(2)$ \\
\hline $\mathrm{C}(21)$ & $27(2)$ & $23(2)$ & $13(2)$ & 2(1) & $-5(2)$ & $-17(2)$ \\
\hline $\mathrm{C}(22)$ & $15(2)$ & $14(2)$ & $18(2)$ & 1(1) & $-5(1)$ & $-7(1)$ \\
\hline $\mathrm{C}(23)$ & $20(2)$ & $15(2)$ & $5(2)$ & $0(1)$ & $-6(1)$ & $-6(1)$ \\
\hline$C(24)$ & $20(2)$ & $18(2)$ & 11(2) & $0(1)$ & $-6(1)$ & $-4(2)$ \\
\hline$C(25)$ & $24(2)$ & $26(2)$ & $16(2)$ & $6(2)$ & $-11(2)$ & $-17(2)$ \\
\hline$C(26)$ & $32(2)$ & $17(2)$ & $19(2)$ & 2(1) & $-9(2)$ & $-14(2)$ \\
\hline $\mathrm{C}(27)$ & $27(2)$ & $14(2)$ & $19(2)$ & $0(1)$ & $-6(2)$ & $-3(2)$ \\
\hline C(28) & $18(2)$ & $19(2)$ & $12(2)$ & 2(1) & $-2(1)$ & $-7(2)$ \\
\hline C(29) & $13(2)$ & $10(2)$ & $19(2)$ & $3(1)$ & $-8(1)$ & $-4(1)$ \\
\hline $\mathrm{C}(30)$ & $19(2)$ & $25(2)$ & $18(2)$ & $5(2)$ & $-7(2)$ & $-13(2)$ \\
\hline $\mathrm{C}(31)$ & $26(2)$ & $35(2)$ & $24(2)$ & $14(2)$ & $-15(2)$ & $-20(2)$ \\
\hline $\mathrm{C}(32)$ & $15(2)$ & $33(2)$ & $42(3)$ & 21(2) & $-14(2)$ & $-14(2)$ \\
\hline $\mathrm{C}(33)$ & $17(2)$ & $20(2)$ & $30(2)$ & $4(2)$ & $1(2)$ & $-4(2)$ \\
\hline $\mathrm{C}(34)$ & $17(2)$ & $19(2)$ & $20(2)$ & 2(2) & $-7(2)$ & $-5(2)$ \\
\hline
\end{tabular}




\subsection{Crystal Structure Determination of $\mathbf{2 e}$}

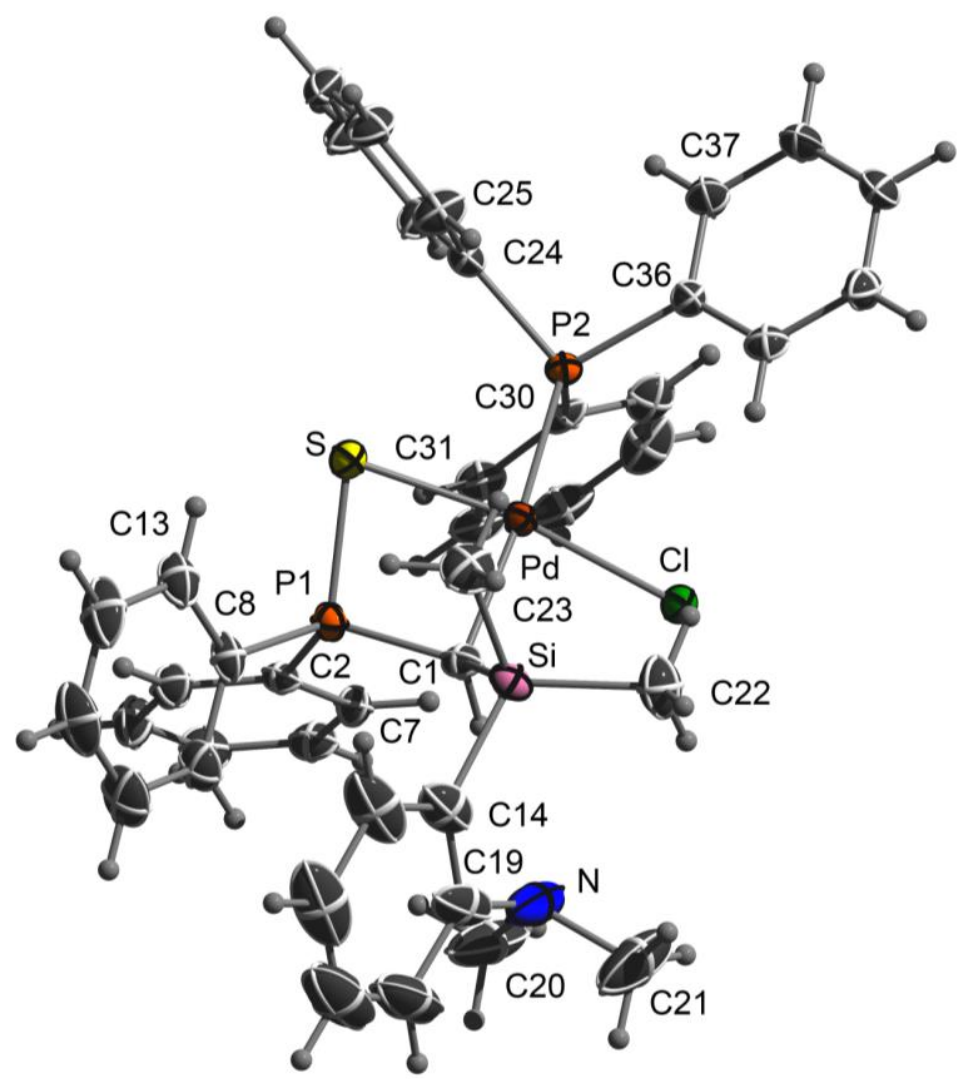

Figure S52. ORTEP Plot of palladium complex 2e. Ellipsoids are drawn at the 50\% probability level

Table S20. Atomic coordinates $\left(\mathrm{x} 10^{4}\right)$ and equivalent isotropic displacement parameters $\left(\AA^{2} \times 10^{3}\right)$ for palladium complex $2 \mathbf{e}$. $U(\mathrm{eq})$ is defined as one third of the trace of the orthogonalized $\mathrm{U}^{\mathrm{ij}}$ tensor.

\begin{tabular}{lllll}
\hline & $\mathrm{X}$ & $\mathrm{y}$ & $\mathrm{Z}$ & $\mathrm{U}(\mathrm{eq})$ \\
\hline $\mathrm{Pd}(1)$ & $7766(1)$ & $4749(1)$ & $2279(1)$ & $17(1)$ \\
$\mathrm{C}(1)$ & $7248(2)$ & $4138(2)$ & $3148(2)$ & $22(1)$ \\
$\mathrm{Cl}(1)$ & $9199(1)$ & $5194(1)$ & $3011(1)$ & $24(1)$ \\
$\mathrm{S}(1)$ & $6374(1)$ & $4083(1)$ & $1652(1)$ & $25(1)$ \\
$\mathrm{P}(1)$ & $6562(1)$ & $3342(1)$ & $2572(1)$ & $20(1)$ \\
$\mathrm{Si}(1)$ & $6668(1)$ & $4978(1)$ & $3680(1)$ & $24(1)$ \\
$\mathrm{N}(1)$ & $7840(3)$ & $3844(2)$ & $4829(2)$ & $51(1)$ \\
$\mathrm{P}(2)$ & $8349(1)$ & $5200(1)$ & $1288(1)$ & $18(1)$ \\
$\mathrm{C}(2)$ & $7216(2)$ & $2259(2)$ & $2516(2)$ & $20(1)$ \\
$\mathrm{C}(3)$ & $6724(2)$ & $1438(2)$ & $2285(2)$ & $28(1)$ \\
$\mathrm{C}(4)$ & $7205(2)$ & $618(2)$ & $2194(2)$ & $32(1)$ \\
$\mathrm{C}(5)$ & $8190(2)$ & $617(2)$ & $2331(2)$ & $31(1)$ \\
$\mathrm{C}(6)$ & $8682(2)$ & $1421(2)$ & $2564(2)$ & $27(1)$ \\
$\mathrm{C}(7)$ & $8212(2)$ & $2244(2)$ & $2661(2)$ & $24(1)$ \\
$\mathrm{C}(8)$ & $5434(2)$ & $2967(2)$ & $2766(2)$ & $24(1)$ \\
$\mathrm{C}(9)$ & $5424(2)$ & $2412(2)$ & $3361(2)$ & $33(1)$ \\
& & & &
\end{tabular}




\begin{tabular}{|c|c|c|c|c|}
\hline $\mathrm{C}(10)$ & $4576(3)$ & 2141(3) & $3539(2)$ & $45(1)$ \\
\hline $\mathrm{C}(11)$ & $3730(3)$ & $2420(3)$ & $3125(2)$ & $49(1)$ \\
\hline$C(13)$ & $4578(2)$ & $3241(2)$ & $2351(2)$ & $34(1)$ \\
\hline $\mathrm{C}(12)$ & $3718(2)$ & 2968(3) & $2539(2)$ & $46(1)$ \\
\hline$C(14)$ & $6208(3)$ & $4287(3)$ & $4382(2)$ & $38(1)$ \\
\hline$C(15)$ & $5246(3)$ & $4219(3)$ & $4413(2)$ & $55(1)$ \\
\hline$C(16)$ & $4945(4)$ & $3612(4)$ & 4918(3) & $72(2)$ \\
\hline$C(17)$ & $5615(6)$ & $3090(4)$ & $5369(3)$ & $84(2)$ \\
\hline$C(18)$ & $6548(5)$ & $3153(3)$ & $5348(2)$ & $77(2)$ \\
\hline$C(19)$ & $6859(4)$ & $3752(3)$ & $4866(2)$ & $51(1)$ \\
\hline $\mathrm{C}(20)$ & $8290(4)$ & 2944(3) & $4726(2)$ & $75(2)$ \\
\hline $\mathrm{C}(21)$ & $8379(4)$ & $4355(4)$ & $5440(3)$ & $84(2)$ \\
\hline $\mathrm{C}(22)$ & $7500(3)$ & $5937(3)$ & $4049(2)$ & $40(1)$ \\
\hline $\mathrm{C}(23)$ & $5619(2)$ & $5550(3)$ & $3122(2)$ & $38(1)$ \\
\hline $\mathrm{C}(24)$ & $7565(2)$ & $5027(2)$ & $429(2)$ & $22(1)$ \\
\hline$C(25)$ & $6690(2)$ & $5468(2)$ & $312(2)$ & $31(1)$ \\
\hline$C(26)$ & $6068(2)$ & $5388(3)$ & $-331(2)$ & $37(1)$ \\
\hline $\mathrm{C}(27)$ & $6321(3)$ & $4857(2)$ & $-863(2)$ & $36(1)$ \\
\hline $\mathrm{C}(28)$ & $7192(3)$ & $4413(3)$ & $-759(2)$ & $41(1)$ \\
\hline $\mathrm{C}(29)$ & $7813(2)$ & $4492(2)$ & $-111(2)$ & $32(1)$ \\
\hline $\mathrm{C}(30)$ & $9356(2)$ & $4420(2)$ & $1264(2)$ & $21(1)$ \\
\hline $\mathrm{C}(31)$ & $9217(2)$ & $3465(2)$ & $1373(2)$ & $26(1)$ \\
\hline $\mathrm{C}(32)$ & $9948(3)$ & $2822(3)$ & $1396(2)$ & $33(1)$ \\
\hline $\mathrm{C}(33)$ & $10833(3)$ & $3130(3)$ & $1326(2)$ & $38(1)$ \\
\hline $\mathrm{C}(34)$ & $10989(2)$ & $4071(3)$ & $1225(2)$ & $38(1)$ \\
\hline$C(35)$ & $10251(2)$ & $4722(3)$ & 1190(2) & $30(1)$ \\
\hline$C(36)$ & $8743(2)$ & $6408(2)$ & $1212(2)$ & $20(1)$ \\
\hline$C(37)$ & $8917(3)$ & $6749(3)$ & $572(2)$ & $38(1)$ \\
\hline $\mathrm{C}(38)$ & $9206(3)$ & $7670(3)$ & $511(2)$ & $45(1)$ \\
\hline C(39) & $9335(3)$ & $8261(2)$ & $1090(2)$ & $37(1)$ \\
\hline$C(40)$ & $9164(2)$ & $7938(2)$ & $1729(2)$ & $26(1)$ \\
\hline$C(41)$ & $8861(2)$ & $7013(2)$ & $1789(2)$ & $21(1)$ \\
\hline
\end{tabular}

Table S21. Anisotropic displacement parameters $\left(\AA^{2} \times 10^{3}\right)$ for palladium complex 2e. The anisotropic displacement factor exponent takes the form: $-2 \pi^{2}\left[\mathrm{~h}^{2} \mathrm{a}^{2} \mathrm{U}^{11}+\ldots+2 \mathrm{~h} \mathrm{k} \mathrm{a} \cdot \mathrm{b} \cdot \mathrm{U}^{12}\right]$.

\begin{tabular}{ccccccc}
\hline & $\mathrm{U}^{11}$ & $\mathrm{U}^{22}$ & $\mathrm{U}^{22}$ & $\mathrm{U}^{23}$ & $\mathrm{U}^{13}$ & $\mathrm{U}^{12}$ \\
\hline $\mathrm{Pd}(1)$ & $17(1)$ & $19(1)$ & $16(1)$ & $3(1)$ & $4(1)$ & $-1(1)$ \\
$\mathrm{C}(1)$ & $19(2)$ & $26(2)$ & $21(2)$ & $6(1)$ & $5(1)$ & $1(1)$ \\
$\mathrm{Cl}(1)$ & $22(1)$ & $27(1)$ & $22(1)$ & $4(1)$ & $1(1)$ & $-4(1)$
\end{tabular}




\begin{tabular}{|c|c|c|c|c|c|c|}
\hline$S(1)$ & $22(1)$ & $27(1)$ & $23(1)$ & $5(1)$ & $-1(1)$ & $-4(1)$ \\
\hline $\mathrm{P}(1)$ & $16(1)$ & $22(1)$ & $22(1)$ & $2(1)$ & $4(1)$ & $-1(1)$ \\
\hline $\operatorname{Si}(1)$ & $26(1)$ & $23(1)$ & $24(1)$ & $2(1)$ & $8(1)$ & $1(1)$ \\
\hline $\mathrm{N}(1)$ & $74(3)$ & $41(2)$ & $30(2)$ & $-6(2)$ & $-11(2)$ & $11(2)$ \\
\hline $\mathrm{P}(2)$ & $20(1)$ & $20(1)$ & $16(1)$ & $0(1)$ & $4(1)$ & $-2(1)$ \\
\hline$C(2)$ & $21(2)$ & $22(2)$ & $19(2)$ & $5(1)$ & $6(1)$ & $0(1)$ \\
\hline$C(3)$ & $24(2)$ & $33(2)$ & $27(2)$ & $1(2)$ & $5(1)$ & $-2(2)$ \\
\hline$C(4)$ & $34(2)$ & $24(2)$ & $37(2)$ & $-6(2)$ & $4(2)$ & $-3(2)$ \\
\hline$C(5)$ & $34(2)$ & $29(2)$ & $30(2)$ & $0(2)$ & $9(2)$ & $7(2)$ \\
\hline$C(6)$ & $20(2)$ & $34(2)$ & $28(2)$ & $6(2)$ & $5(1)$ & $5(1)$ \\
\hline$C(7)$ & $19(2)$ & $27(2)$ & $24(2)$ & $2(1)$ & $2(1)$ & $-2(1)$ \\
\hline $\mathrm{C}(8)$ & $18(2)$ & $20(2)$ & $35(2)$ & $-3(2)$ & $7(1)$ & $-2(1)$ \\
\hline$C(9)$ & $27(2)$ & $39(2)$ & $34(2)$ & $-1(2)$ & $11(2)$ & $-6(2)$ \\
\hline$C(10)$ & $35(2)$ & $55(3)$ & $50(3)$ & $-1(2)$ & $20(2)$ & $-15(2)$ \\
\hline $\mathrm{C}(11)$ & $34(2)$ & $47(3)$ & $74(3)$ & $-7(2)$ & $29(2)$ & $-13(2)$ \\
\hline $\mathrm{C}(13)$ & $25(2)$ & $26(2)$ & $50(2)$ & $1(2)$ & $3(2)$ & $-1(2)$ \\
\hline$C(12)$ & $19(2)$ & $39(2)$ & $78(3)$ & $-4(2)$ & $4(2)$ & $0(2)$ \\
\hline $\mathrm{C}(14)$ & $60(3)$ & $29(2)$ & $33(2)$ & $-5(2)$ & $24(2)$ & $-2(2)$ \\
\hline$C(15)$ & $77(3)$ & $41(2)$ & $63(3)$ & $-14(2)$ & $54(3)$ & $-11(2)$ \\
\hline$C(16)$ & $103(4)$ & $56(3)$ & $77(4)$ & $-32(3)$ & $68(3)$ & $-29(3)$ \\
\hline$C(17)$ & 159(7) & $54(3)$ & $52(3)$ & $-7(3)$ & $55(4)$ & $-28(4)$ \\
\hline$C(18)$ & $156(6)$ & $46(3)$ & $34(3)$ & $5(2)$ & $28(3)$ & $-15(3)$ \\
\hline$C(19)$ & $108(4)$ & $27(2)$ & $20(2)$ & $-6(2)$ & $14(2)$ & $-3(2)$ \\
\hline $\mathrm{C}(20)$ & $128(5)$ & $50(3)$ & $36(3)$ & $0(2)$ & $-14(3)$ & $35(3)$ \\
\hline $\mathrm{C}(21)$ & $105(4)$ & $78(4)$ & $51(3)$ & $-29(3)$ & $-29(3)$ & $24(3)$ \\
\hline $\mathrm{C}(22)$ & $40(2)$ & $33(2)$ & $50(2)$ & $-11(2)$ & $13(2)$ & $-4(2)$ \\
\hline $\mathrm{C}(23)$ & $36(2)$ & $33(2)$ & $43(2)$ & $1(2)$ & $6(2)$ & $11(2)$ \\
\hline $\mathrm{C}(24)$ & $24(2)$ & $20(2)$ & $20(2)$ & $3(1)$ & $3(1)$ & $-4(1)$ \\
\hline$C(25)$ & $30(2)$ & $39(2)$ & $25(2)$ & $-3(2)$ & $2(2)$ & $7(2)$ \\
\hline $\mathrm{C}(26)$ & $30(2)$ & $42(2)$ & $34(2)$ & $9(2)$ & $-3(2)$ & $5(2)$ \\
\hline $\mathrm{C}(27)$ & $41(2)$ & $31(2)$ & $27(2)$ & $2(2)$ & $-14(2)$ & $-6(2)$ \\
\hline $\mathrm{C}(28)$ & $51(2)$ & $36(2)$ & $29(2)$ & $-11(2)$ & $-8(2)$ & $6(2)$ \\
\hline $\mathrm{C}(29)$ & $34(2)$ & $31(2)$ & $27(2)$ & $-6(2)$ & $-3(2)$ & $4(2)$ \\
\hline $\mathrm{C}(30)$ & $24(2)$ & $29(2)$ & $10(2)$ & $-3(1)$ & $2(1)$ & 1(1) \\
\hline $\mathrm{C}(31)$ & $27(2)$ & $30(2)$ & $22(2)$ & $2(2)$ & $4(1)$ & $5(1)$ \\
\hline$C(32)$ & $42(2)$ & $35(2)$ & $20(2)$ & $1(2)$ & $-1(2)$ & $13(2)$ \\
\hline $\mathrm{C}(33)$ & $37(2)$ & $52(3)$ & $23(2)$ & $-8(2)$ & $3(2)$ & $19(2)$ \\
\hline $\mathrm{C}(34)$ & $22(2)$ & $62(3)$ & $32(2)$ & $-14(2)$ & $11(2)$ & $-1(2)$ \\
\hline $\mathrm{C}(35)$ & $30(2)$ & $37(2)$ & $23(2)$ & $-8(2)$ & $10(1)$ & $-1(2)$ \\
\hline
\end{tabular}




\begin{tabular}{lllllll}
$\mathrm{C}(36)$ & $20(2)$ & $21(2)$ & $18(2)$ & $2(1)$ & $1(1)$ & $-3(1)$ \\
$\mathrm{C}(37)$ & $57(2)$ & $37(2)$ & $19(2)$ & $-2(2)$ & $8(2)$ & $-19(2)$ \\
$\mathrm{C}(38)$ & $74(3)$ & $39(2)$ & $22(2)$ & $6(2)$ & $4(2)$ & $-25(2)$ \\
$\mathrm{C}(39)$ & $52(2)$ & $24(2)$ & $30(2)$ & $8(2)$ & $-3(2)$ & $-12(2)$ \\
$\mathrm{C}(40)$ & $31(2)$ & $20(2)$ & $23(2)$ & $-2(1)$ & $0(1)$ & $1(1)$ \\
$\mathrm{C}(41)$ & $20(2)$ & $24(2)$ & $21(2)$ & $2(1)$ & $4(1)$ & $4(1)$ \\
\hline
\end{tabular}

\subsection{Crystal Structure Determination of $\mathbf{2 f}$}

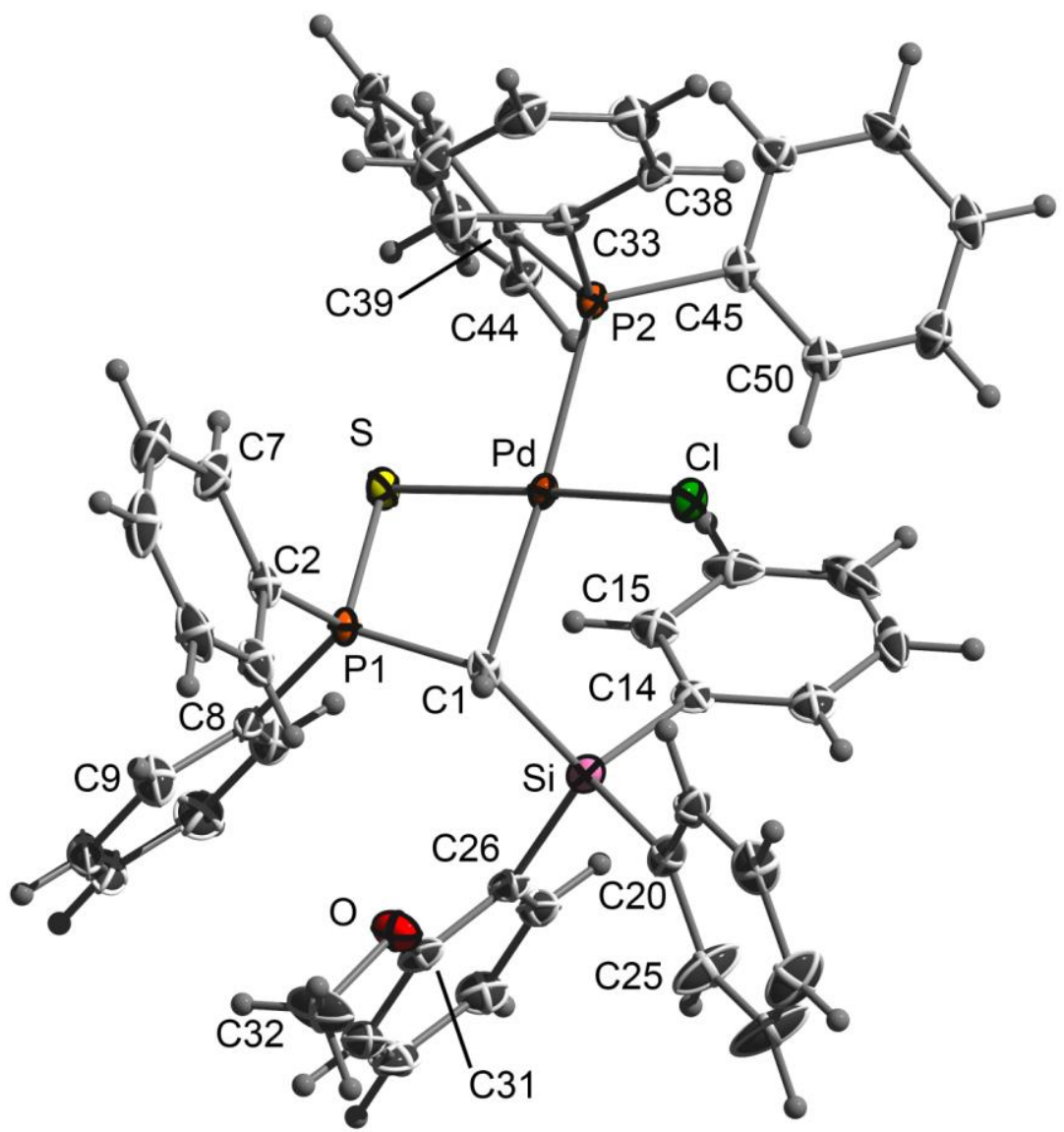

Figure S53. ORTEP Plot of palladium complex 2f. Ellipsoids are drawn at the 50\% probability level

Table S22. Atomic coordinates $\left(\mathrm{x} 10^{4}\right)$ and equivalent isotropic displacement parameters $\left(\AA^{2} \times 10^{3}\right)$ for palladium complex $\mathbf{2 f}$. $U(e q)$ is defined as one third of the trace of the orthogonalized $\mathrm{U}^{\mathrm{ij}}$ tensor.

\begin{tabular}{ccccc}
\hline & $\mathrm{X}$ & $\mathrm{y}$ & $\mathrm{z}$ & $\mathrm{U}(\mathrm{eq})$ \\
\hline $\mathrm{Pd}(1)$ & $7282(1)$ & $9610(1)$ & $7423(1)$ & $11(1)$ \\
$\mathrm{P}(1)$ & $8413(1)$ & $8399(1)$ & $6435(1)$ & $13(1)$ \\
$\mathrm{Cl}(1)$ & $5038(1)$ & $9929(1)$ & $7851(1)$ & $17(1)$ \\
$\mathrm{S}(1)$ & $9426(1)$ & $9288(1)$ & $6859(1)$ & $16(1)$
\end{tabular}




\begin{tabular}{|c|c|c|c|c|}
\hline $\mathrm{Si}(1)$ & $7096(1)$ & $7082(1)$ & $8169(1)$ & $13(1)$ \\
\hline $\mathrm{O}(1)$ & $7216(2)$ & $5942(2)$ & $6948(1)$ & $22(1)$ \\
\hline $\mathrm{C}(1)$ & $7097(3)$ & $8169(2)$ & $7184(2)$ & 11(1) \\
\hline $\mathrm{C}(2)$ & $7640(3)$ & $9110(2)$ & $5459(2)$ & $14(1)$ \\
\hline $\mathrm{P}(2)$ & $7594(1)$ & $11146(1)$ & $7611(1)$ & $12(1)$ \\
\hline $\mathrm{C}(3)$ & $6645(3)$ & $8707(3)$ & $5181(2)$ & $20(1)$ \\
\hline $\mathrm{C}(4)$ & $6054(3)$ & $9235(3)$ & $4436(2)$ & $27(1)$ \\
\hline$C(5)$ & $6433(3)$ & $10179(3)$ & $3967(2)$ & $29(1)$ \\
\hline$C(6)$ & $7400(3)$ & $10592(3)$ & $4244(2)$ & $27(1)$ \\
\hline$C(7)$ & $8009(3)$ & $10059(3)$ & 4988(2) & $22(1)$ \\
\hline $\mathrm{C}(8)$ & $9612(3)$ & $7346(2)$ & $6231(2)$ & $14(1)$ \\
\hline $\mathrm{C}(9)$ & $9540(3)$ & $7006(3)$ & $5545(2)$ & $22(1)$ \\
\hline$C(10)$ & $10474(3)$ & $6197(3)$ & $5424(2)$ & $26(1)$ \\
\hline $\mathrm{C}(11)$ & 11492(3) & $5728(3)$ & $5974(2)$ & $27(1)$ \\
\hline$C(12)$ & 11587(3) & $6070(3)$ & $6649(2)$ & $25(1)$ \\
\hline$C(13)$ & $10647(3)$ & $6869(2)$ & $6779(2)$ & $18(1)$ \\
\hline $\mathrm{C}(14)$ & $7575(3)$ & $7473(2)$ & $9065(2)$ & $15(1)$ \\
\hline$C(15)$ & $8829(3)$ & $7793(2)$ & $9075(2)$ & 19(1) \\
\hline$C(16)$ & $9175(4)$ & $8100(2)$ & $9727(2)$ & $25(1)$ \\
\hline$C(17)$ & $8279(4)$ & $8092(3)$ & $10387(2)$ & $30(1)$ \\
\hline $\mathrm{C}(18)$ & $7038(4)$ & $7780(3)$ & $10396(2)$ & $29(1)$ \\
\hline$C(19)$ & $6697(3)$ & $7467(2)$ & $9743(2)$ & $21(1)$ \\
\hline$C(20)$ & $5346(3)$ & $6715(2)$ & $8363(2)$ & $17(1)$ \\
\hline $\mathrm{C}(21)$ & 4199(3) & $7426(3)$ & $8100(2)$ & $18(1)$ \\
\hline $\mathrm{C}(22)$ & $2922(3)$ & $7146(3)$ & $8273(2)$ & $26(1)$ \\
\hline$C(23)$ & 2759(4) & $6139(3)$ & $8712(2)$ & $36(1)$ \\
\hline$C(24)$ & $3873(4)$ & $5412(3)$ & $8975(3)$ & $49(1)$ \\
\hline$C(25)$ & $5154(4)$ & $5696(3)$ & $8806(2)$ & $36(1)$ \\
\hline$C(26)$ & $8281(3)$ & $5877(2)$ & $8139(2)$ & $15(1)$ \\
\hline $\mathrm{C}(27)$ & $9229(3)$ & $5369(2)$ & $8736(2)$ & $19(1)$ \\
\hline$C(28)$ & $10076(3)$ & $4465(3)$ & $8708(2)$ & $22(1)$ \\
\hline C(29) & $9967(3)$ & $4047(3)$ & $8079(2)$ & $25(1)$ \\
\hline $\mathrm{C}(30)$ & $9025(3)$ & $4521(2)$ & $7475(2)$ & $22(1)$ \\
\hline $\mathrm{C}(31)$ & $8192(3)$ & $5419(2)$ & $7514(2)$ & $18(1)$ \\
\hline$C(32)$ & $6934(4)$ & $5443(3)$ & $6370(2)$ & $36(1)$ \\
\hline$C(33)$ & $6535(3)$ & $12206(2)$ & $6904(2)$ & $13(1)$ \\
\hline$C(34)$ & $6714(3)$ & $12351(3)$ & $6056(2)$ & $21(1)$ \\
\hline$C(35)$ & $5858(3)$ & 13094(3) & $5502(2)$ & $24(1)$ \\
\hline$C(36)$ & $4787(3)$ & $13683(3)$ & $5790(2)$ & $24(1)$ \\
\hline
\end{tabular}




\begin{tabular}{rrrrr}
$\mathrm{C}(37)$ & $4583(3)$ & $13536(3)$ & $6624(2)$ & $23(1)$ \\
$\mathrm{C}(38)$ & $5451(3)$ & $12805(2)$ & $7182(2)$ & $18(1)$ \\
$\mathrm{C}(39)$ & $9316(3)$ & $11435(2)$ & $7404(2)$ & $13(1)$ \\
$\mathrm{C}(40)$ & $9725(3)$ & $12267(2)$ & $6783(2)$ & $15(1)$ \\
$\mathrm{C}(41)$ & $11082(3)$ & $12390(3)$ & $6677(2)$ & $20(1)$ \\
$\mathrm{C}(42)$ & $12030(3)$ & $11687(3)$ & $7180(2)$ & $20(1)$ \\
$\mathrm{C}(43)$ & $11630(3)$ & $10844(2)$ & $7800(2)$ & $19(1)$ \\
$\mathrm{C}(44)$ & $10287(3)$ & $10721(2)$ & $7914(2)$ & $16(1)$ \\
$\mathrm{C}(45)$ & $7225(3)$ & $11300(2)$ & $8631(2)$ & $14(1)$ \\
$\mathrm{C}(46)$ & $7476(3)$ & $12197(2)$ & $8798(2)$ & $19(1)$ \\
$\mathrm{C}(47)$ & $7232(3)$ & $12294(3)$ & $9583(2)$ & $22(1)$ \\
$\mathrm{C}(48)$ & $6757(3)$ & $11501(3)$ & $10213(2)$ & $22(1)$ \\
$\mathrm{C}(49)$ & $6515(3)$ & $10617(3)$ & $10048(2)$ & $20(1)$ \\
$\mathrm{C}(50)$ & $6748(3)$ & $10511(2)$ & $9266(2)$ & $14(1)$ \\
\hline
\end{tabular}

Table S23. Anisotropic displacement parameters $\left(\AA^{2} \times 10^{3}\right)$ for palladium complex 2f. The anisotropic displacement factor exponent takes the form: $-2 \pi^{2}\left[\mathrm{~h}^{2} \mathrm{a}^{2} \mathrm{U}^{11}+\ldots+2 \mathrm{~h} \mathrm{k} \mathrm{a} \cdot \mathrm{b} \cdot \mathrm{U}^{12}\right]$.

\begin{tabular}{ccccccc}
\hline & $\mathrm{U}^{11}$ & $\mathrm{U}^{22}$ & $\mathrm{U}^{22}$ & $\mathrm{U}^{23}$ & $\mathrm{U}^{13}$ & $\mathrm{U}^{12}$ \\
\hline $\mathrm{Pd}(1)$ & $8(1)$ & $13(1)$ & $12(1)$ & $-3(1)$ & $0(1)$ & $-2(1)$ \\
$\mathrm{P}(1)$ & $11(1)$ & $15(1)$ & $13(1)$ & $-4(1)$ & $0(1)$ & $-1(1)$ \\
$\mathrm{Cl}(1)$ & $8(1)$ & $21(1)$ & $21(1)$ & $-7(1)$ & $2(1)$ & $-2(1)$ \\
$\mathrm{S}(1)$ & $10(1)$ & $21(1)$ & $20(1)$ & $-10(1)$ & $2(1)$ & $-3(1)$ \\
$\mathrm{Si}(1)$ & $11(1)$ & $15(1)$ & $13(1)$ & $-3(1)$ & $-2(1)$ & $-2(1)$ \\
$\mathrm{O}(1)$ & $27(1)$ & $20(1)$ & $21(1)$ & $-7(1)$ & $-10(1)$ & $-3(1)$ \\
$\mathrm{C}(1)$ & $6(2)$ & $15(2)$ & $14(2)$ & $-7(1)$ & $-2(1)$ & $0(1)$ \\
$\mathrm{C}(2)$ & $12(2)$ & $18(2)$ & $11(2)$ & $-4(1)$ & $-1(1)$ & $3(1)$ \\
$\mathrm{P}(2)$ & $10(1)$ & $13(1)$ & $12(1)$ & $-3(1)$ & $0(1)$ & $-1(1)$ \\
$\mathrm{C}(3)$ & $21(2)$ & $21(2)$ & $18(2)$ & $-8(2)$ & $-3(2)$ & $3(1)$ \\
$\mathrm{C}(4)$ & $24(2)$ & $38(2)$ & $21(2)$ & $-16(2)$ & $-5(2)$ & $2(2)$ \\
$\mathrm{C}(5)$ & $22(2)$ & $41(3)$ & $15(2)$ & $-4(2)$ & $-1(2)$ & $9(2)$ \\
$\mathrm{C}(6)$ & $21(2)$ & $28(2)$ & $22(2)$ & $2(2)$ & $5(2)$ & $2(2)$ \\
$\mathrm{C}(7)$ & $14(2)$ & $24(2)$ & $22(2)$ & $-2(2)$ & $2(2)$ & $0(1)$ \\
$\mathrm{C}(8)$ & $15(2)$ & $13(2)$ & $12(2)$ & $-2(1)$ & $5(1)$ & $-2(1)$ \\
$\mathrm{C}(9)$ & $22(2)$ & $24(2)$ & $18(2)$ & $-5(2)$ & $-3(2)$ & $5(2)$ \\
$\mathrm{C}(10)$ & $34(2)$ & $23(2)$ & $17(2)$ & $-5(2)$ & $5(2)$ & $3(2)$ \\
$\mathrm{C}(11)$ & $28(2)$ & $18(2)$ & $27(2)$ & $-4(2)$ & $8(2)$ & $7(2)$ \\
$\mathrm{C}(12)$ & $17(2)$ & $23(2)$ & $30(2)$ & $-5(2)$ & $-3(2)$ & $6(2)$ \\
$\mathrm{C}(13)$ & $15(2)$ & $21(2)$ & $18(2)$ & $-5(2)$ & $-1(1)$ & $-2(1)$ \\
$\mathrm{C}(14)$ & $19(2)$ & $9(2)$ & $14(2)$ & $0(1)$ & $-5(1)$ & $4(1)$ \\
& & & & & &
\end{tabular}




\begin{tabular}{|c|c|c|c|c|c|c|}
\hline $\mathrm{C}(15)$ & $19(2)$ & $16(2)$ & $21(2)$ & $-4(2)$ & $-6(2)$ & $1(1)$ \\
\hline$C(16)$ & $32(2)$ & $14(2)$ & $28(2)$ & $-2(2)$ & $-16(2)$ & $-1(2)$ \\
\hline$C(17)$ & $50(3)$ & $17(2)$ & $23(2)$ & $-8(2)$ & $-19(2)$ & $7(2)$ \\
\hline $\mathrm{C}(18)$ & $44(2)$ & $24(2)$ & $13(2)$ & $-4(2)$ & $0(2)$ & $8(2)$ \\
\hline $\mathrm{C}(19)$ & $22(2)$ & $15(2)$ & $21(2)$ & $-1(2)$ & $-4(2)$ & $3(1)$ \\
\hline $\mathrm{C}(20)$ & $16(2)$ & $23(2)$ & $13(2)$ & $-4(1)$ & $-2(1)$ & $-9(1)$ \\
\hline $\mathrm{C}(21)$ & $18(2)$ & $21(2)$ & $17(2)$ & $-6(2)$ & $1(2)$ & $-6(1)$ \\
\hline $\mathrm{C}(22)$ & $16(2)$ & $37(2)$ & $28(2)$ & $-15(2)$ & $-3(2)$ & $-5(2)$ \\
\hline$C(23)$ & 21(2) & $48(3)$ & $38(2)$ & $-4(2)$ & $-1(2)$ & $-18(2)$ \\
\hline$C(24)$ & $39(3)$ & $38(3)$ & $59(3)$ & $12(2)$ & $-5(2)$ & $-25(2)$ \\
\hline$C(25)$ & $20(2)$ & $31(2)$ & $44(3)$ & 11(2) & $-6(2)$ & $-9(2)$ \\
\hline$C(26)$ & $15(2)$ & $13(2)$ & $18(2)$ & $-3(1)$ & $0(1)$ & $-5(1)$ \\
\hline $\mathrm{C}(27)$ & $21(2)$ & $16(2)$ & $20(2)$ & $-5(2)$ & $-1(2)$ & $-5(1)$ \\
\hline$C(28)$ & $18(2)$ & $19(2)$ & $27(2)$ & $-3(2)$ & $-5(2)$ & $-1(1)$ \\
\hline $\mathrm{C}(29)$ & $24(2)$ & $13(2)$ & $33(2)$ & $-3(2)$ & $1(2)$ & $1(1)$ \\
\hline$C(30)$ & $28(2)$ & $17(2)$ & $22(2)$ & $-6(2)$ & $1(2)$ & $-7(2)$ \\
\hline $\mathrm{C}(31)$ & $18(2)$ & $15(2)$ & $20(2)$ & $-2(2)$ & $-2(2)$ & $-8(1)$ \\
\hline $\mathrm{C}(32)$ & 49(3) & $30(2)$ & $35(2)$ & $-14(2)$ & $-21(2)$ & $-6(2)$ \\
\hline $\mathrm{C}(33)$ & $9(2)$ & $12(2)$ & $18(2)$ & $-1(1)$ & $-5(1)$ & $-3(1)$ \\
\hline $\mathrm{C}(34)$ & $14(2)$ & $27(2)$ & $21(2)$ & $-7(2)$ & $-5(2)$ & 1(1) \\
\hline$C(35)$ & $24(2)$ & $30(2)$ & $16(2)$ & $-1(2)$ & $-4(2)$ & $-6(2)$ \\
\hline$C(36)$ & $20(2)$ & $22(2)$ & $26(2)$ & $1(2)$ & $-7(2)$ & $-1(2)$ \\
\hline $\mathrm{C}(37)$ & $17(2)$ & $20(2)$ & $30(2)$ & $-6(2)$ & $-3(2)$ & $3(1)$ \\
\hline $\mathrm{C}(38)$ & $14(2)$ & $19(2)$ & $17(2)$ & $-2(1)$ & $1(1)$ & $-2(1)$ \\
\hline C(39) & $12(2)$ & $16(2)$ & $14(2)$ & $-9(1)$ & $0(1)$ & $-4(1)$ \\
\hline $\mathrm{C}(40)$ & $13(2)$ & $18(2)$ & $16(2)$ & $-5(1)$ & $-3(1)$ & $-3(1)$ \\
\hline $\mathrm{C}(41)$ & $23(2)$ & $21(2)$ & $16(2)$ & $-5(2)$ & $5(2)$ & $-13(2)$ \\
\hline $\mathrm{C}(42)$ & 11(2) & $29(2)$ & $23(2)$ & $-13(2)$ & $1(2)$ & $-6(1)$ \\
\hline $\mathrm{C}(43)$ & $14(2)$ & $22(2)$ & $21(2)$ & $-7(2)$ & $-1(2)$ & $1(1)$ \\
\hline C(44) & $12(2)$ & $17(2)$ & $18(2)$ & $-2(1)$ & $-2(1)$ & $-3(1)$ \\
\hline $\mathrm{C}(45)$ & $7(2)$ & $19(2)$ & $14(2)$ & $-5(1)$ & $-2(1)$ & $2(1)$ \\
\hline $\mathrm{C}(46)$ & $17(2)$ & $19(2)$ & $21(2)$ & $-8(2)$ & $-1(2)$ & $-3(1)$ \\
\hline $\mathrm{C}(47)$ & $19(2)$ & $23(2)$ & $30(2)$ & $-16(2)$ & $-4(2)$ & $-1(2)$ \\
\hline $\mathrm{C}(48)$ & $18(2)$ & $30(2)$ & $17(2)$ & $-10(2)$ & $-2(2)$ & $2(2)$ \\
\hline C(49) & $19(2)$ & $25(2)$ & $15(2)$ & $-3(2)$ & $-1(2)$ & $-4(1)$ \\
\hline $\mathrm{C}(50)$ & $9(2)$ & $18(2)$ & $16(2)$ & $-5(1)$ & $-3(1)$ & $0(1)$ \\
\hline
\end{tabular}




\subsection{Crystal Structure Determination of 3}

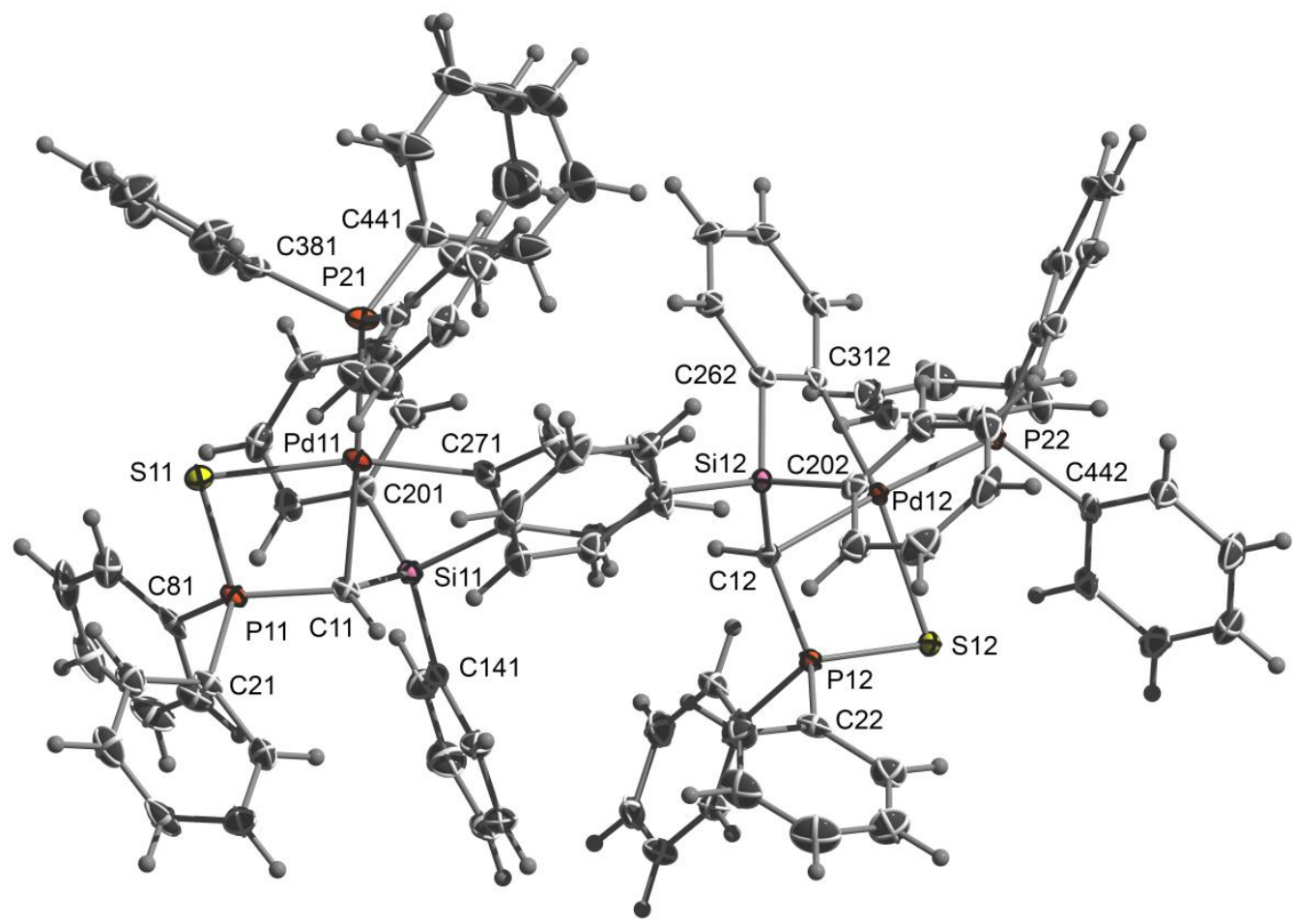

Figure S54. ORTEP Plot of palladium complex 3. Ellipsoids are drawn at the $50 \%$ probability level

Table S24. Atomic coordinates $\left(\mathrm{x} 10^{4}\right)$ and equivalent isotropic displacement parameters $\left(\AA^{2} \times 10^{3}\right)$ for palladium complex 3 . $U(e q)$ is defined as one third of the trace of the orthogonalized $U^{i j}$ tensor.

\begin{tabular}{llrrr}
\hline & $\mathrm{X}$ & $\mathrm{y}$ & $\mathrm{Z}$ & $\mathrm{U}(\mathrm{eq})$ \\
\hline Pd11 & $6502(1)$ & $484(1)$ & $9056(1)$ & $13(1)$ \\
S11 & $6788(1)$ & $-489(1)$ & $9823(1)$ & $18(1)$ \\
P11 & $6400(1)$ & $894(1)$ & $10073(1)$ & $12(1)$ \\
P21 & $6861(1)$ & $-855(1)$ & $8549(1)$ & $16(1)$ \\
Si11 & $6473(1)$ & $3033(1)$ & $9288(1)$ & $12(1)$ \\
C11 & $6198(1)$ & $1700(2)$ & $9555(1)$ & $12(1)$ \\
C21 & $5844(1)$ & $460(2)$ & $10402(1)$ & $14(1)$ \\
C31 & $5387(1)$ & $1104(2)$ & $10371(1)$ & $23(1)$ \\
C41 & $7974(1)$ & $775(3)$ & $10642(1)$ & $28(1)$ \\
C51 & $5018(1)$ & $-185(2)$ & $10945(1)$ & $25(1)$ \\
C61 & $5468(1)$ & $-831(2)$ & $10976(1)$ & $25(1)$ \\
C71 & $5881(1)$ & $-519(2)$ & $10706(1)$ & $21(1)$ \\
C81 & $6809(1)$ & $1716(2)$ & $10512(1)$ & $16(1)$ \\
C91 & $6638(1)$ & $2798(2)$ & $10681(1)$ & $22(1)$ \\
C101 & $6966(1)$ & $3462(3)$ & $10990(1)$ & $30(1)$ \\
C111 & $7464(1)$ & $3068(3)$ & $11124(1)$ & $32(1)$ \\
C121 & $7630(1)$ & $1997(3)$ & $10965(1)$ & $30(1)$ \\
\hline
\end{tabular}




\begin{tabular}{|c|c|c|c|c|}
\hline C131 & $7304(1)$ & $1314(2)$ & $10659(1)$ & $23(1)$ \\
\hline C141 & $6227(1)$ & $4457(2)$ & $9537(1)$ & $15(1)$ \\
\hline $\mathrm{C} 151$ & $5696(1)$ & $4655(2)$ & $9547(1)$ & $21(1)$ \\
\hline C161 & $5501(1)$ & $5717(2)$ & $9705(1)$ & $27(1)$ \\
\hline $\mathrm{C} 171$ & $5836(1)$ & $6599(2)$ & $9865(1)$ & $28(1)$ \\
\hline C181 & $6363(1)$ & $6421(2)$ & $9867(1)$ & $29(1)$ \\
\hline C191 & $6555(1)$ & $5367(2)$ & $9701(1)$ & $24(1)$ \\
\hline C201 & $7202(1)$ & $3100(2)$ & $9308(1)$ & $14(1)$ \\
\hline $\mathrm{C} 211$ & $7441(1)$ & $3038(2)$ & $8871(1)$ & $23(1)$ \\
\hline $\mathrm{C} 221$ & $7975(1)$ & $3139(3)$ & $8853(1)$ & $30(1)$ \\
\hline $\mathrm{C} 231$ & $8284(1)$ & $3282(2)$ & $9278(1)$ & $24(1)$ \\
\hline C241 & $8059(1)$ & $3333(2)$ & $9718(1)$ & $21(1)$ \\
\hline $\mathrm{C} 251$ & $7524(1)$ & $3267(2)$ & $9732(1)$ & $18(1)$ \\
\hline C261 & $6212(1)$ & $2777(2)$ & $8646(1)$ & $15(1)$ \\
\hline $\mathrm{C} 271$ & $6190(1)$ & $1566(2)$ & $8529(1)$ & $17(1)$ \\
\hline $\mathrm{C} 281$ & $5953(1)$ & $1248(3)$ & $8077(1)$ & $28(1)$ \\
\hline C291 & $5767(1)$ & 2089(3) & $7744(1)$ & $30(1)$ \\
\hline $\mathrm{C} 301$ & $5811(1)$ & $3267(2)$ & $7853(1)$ & $23(1)$ \\
\hline C311 & $6029(1)$ & $3614(2)$ & $8306(1)$ & $18(1)$ \\
\hline C321 & $6368(1)$ & $-1877(2)$ & $8297(1)$ & $16(1)$ \\
\hline C331 & $6306(1)$ & $-2194(2)$ & $7811(1)$ & $26(1)$ \\
\hline C341 & $5909(1)$ & $-2942(2)$ & $7652(1)$ & $29(1)$ \\
\hline C351 & $5567(1)$ & $-3364(2)$ & $7966(1)$ & $26(1)$ \\
\hline C361 & $5622(1)$ & $-3050(3)$ & $8453(1)$ & $29(1)$ \\
\hline C371 & $6020(1)$ & $-2311(2)$ & $8619(1)$ & $26(1)$ \\
\hline C381 & $7351(1)$ & $-1781(2)$ & $8873(1)$ & $18(1)$ \\
\hline C391 & $7802(1)$ & $-1239(3)$ & $9059(1)$ & $34(1)$ \\
\hline $\mathrm{C} 401$ & $8210(1)$ & $-1891(3)$ & $9268(1)$ & $38(1)$ \\
\hline C411 & $8167(1)$ & $-3087(3)$ & 9313(1) & $30(1)$ \\
\hline C421 & $7720(1)$ & $-3633(3)$ & $9137(1)$ & $38(1)$ \\
\hline $\mathrm{C} 431$ & $7311(1)$ & $-2983(2)$ & $8917(1)$ & $31(1)$ \\
\hline $\mathrm{C} 441$ & $7223(1)$ & $-429(2)$ & $8026(1)$ & $23(1)$ \\
\hline C45B1 & $7644(5)$ & $-954(11)$ & $7867(6)$ & $27(3)$ \\
\hline C451 & $7520(5)$ & $-1321(9)$ & $7816(5)$ & $37(3)$ \\
\hline $\mathrm{C} 461$ & $7850(1)$ & $-894(3)$ & $7443(1)$ & $43(1)$ \\
\hline C471 & $7875(3)$ & $145(6)$ & $7314(2)$ & $38(1)$ \\
\hline C481 & $7602(3)$ & $980(6)$ & $7546(3)$ & $58(2)$ \\
\hline C491 & $7287(2)$ & $689(5)$ & $7919(2)$ & $39(1)$ \\
\hline C47B1 & $7647(4)$ & $60(9)$ & 7111(3) & $38(1)$ \\
\hline
\end{tabular}




\begin{tabular}{|c|c|c|c|c|}
\hline C48B 1 & $7198(3)$ & $612(6)$ & $7209(3)$ & $38(2)$ \\
\hline C49B1 & $6974(3)$ & $396(6)$ & $7642(3)$ & $39(1)$ \\
\hline $\operatorname{Pd} 12$ & 4481(1) & 2196(1) & $6667(1)$ & $10(1)$ \\
\hline S22 & $3760(1)$ & $3401(1)$ & $6908(1)$ & $14(1)$ \\
\hline $\mathrm{P} 12$ & $3803(1)$ & $2232(1)$ & $7460(1)$ & $11(1)$ \\
\hline $\mathrm{P} 22$ & $4680(1)$ & $3326(1)$ & $6014(1)$ & $12(1)$ \\
\hline Si12 & $4248(1)$ & $-240(1)$ & $7032(1)$ & $12(1)$ \\
\hline $\mathrm{C} 12$ & $4283(1)$ & $1239(2)$ & $7306(1)$ & $12(1)$ \\
\hline $\mathrm{C} 22$ & $3178(1)$ & $1617(2)$ & $7569(1)$ & $15(1)$ \\
\hline C32 & $2728(1)$ & $2111(2)$ & $7359(1)$ & $23(1)$ \\
\hline $\mathrm{C} 42$ & $2247(1)$ & $1681(3)$ & $7469(1)$ & $32(1)$ \\
\hline C52 & $2219(1)$ & $762(3)$ & 7791(1) & $36(1)$ \\
\hline C62 & 2664(1) & $259(3)$ & $8006(1)$ & $32(1)$ \\
\hline C72 & $3148(1)$ & $683(2)$ & $7895(1)$ & $25(1)$ \\
\hline $\mathrm{C} 82$ & $4007(1)$ & $2955(2)$ & $8031(1)$ & $14(1)$ \\
\hline C92 & $3672(1)$ & $3091(2)$ & $8403(1)$ & $22(1)$ \\
\hline C102 & $3845(1)$ & $3644(2)$ & $8836(1)$ & $26(1)$ \\
\hline $\mathrm{C} 112$ & $4346(1)$ & $4043(2)$ & 8901(1) & $23(1)$ \\
\hline $\mathrm{C} 122$ & 4681(1) & $3922(2)$ & $8528(1)$ & $23(1)$ \\
\hline C132 & $4509(1)$ & $3383(2)$ & $8096(1)$ & $17(1)$ \\
\hline C142 & $4219(1)$ & $-1475(2)$ & $7483(1)$ & $13(1)$ \\
\hline C152 & $4151(1)$ & $-2632(2)$ & $7322(1)$ & $18(1)$ \\
\hline C162 & $4125(1)$ & $-3554(2)$ & $7647(1)$ & $25(1)$ \\
\hline $\mathrm{C} 172$ & $4153(1)$ & $-3323(3)$ & $8143(1)$ & $30(1)$ \\
\hline C182 & $4225(1)$ & $-2199(3)$ & $8310(1)$ & $28(1)$ \\
\hline C192 & $4267(1)$ & $-1287(2)$ & 7984(1) & $20(1)$ \\
\hline C202 & $3693(1)$ & $-502(2)$ & $6561(1)$ & $15(1)$ \\
\hline C212 & $3769(1)$ & $-438(2)$ & $6063(1)$ & $20(1)$ \\
\hline $\mathrm{C} 222$ & $3373(1)$ & $-701(2)$ & $5714(1)$ & $27(1)$ \\
\hline $\mathrm{C} 232$ & 2893(1) & $-1031(2)$ & $5852(1)$ & $30(1)$ \\
\hline C242 & 2811(1) & $-1116(3)$ & $6343(1)$ & $32(1)$ \\
\hline $\mathrm{C} 252$ & $3206(1)$ & $-854(2)$ & $6693(1)$ & $23(1)$ \\
\hline C262 & $4885(1)$ & $-206(2)$ & $6748(1)$ & $13(1)$ \\
\hline $\mathrm{C} 272$ & $5236(1)$ & $-1135(2)$ & $6741(1)$ & $18(1)$ \\
\hline C282 & $5724(1)$ & $-969(2)$ & $6568(1)$ & $21(1)$ \\
\hline C292 & $5853(1)$ & $117(2)$ & $6386(1)$ & $17(1)$ \\
\hline C302 & $5504(1)$ & $1034(2)$ & $6377(1)$ & $14(1)$ \\
\hline C312 & $5016(1)$ & $912(2)$ & $6569(1)$ & $12(1)$ \\
\hline C322 & $4824(1)$ & $2555(2)$ & $5457(1)$ & $13(1)$ \\
\hline
\end{tabular}




\begin{tabular}{lllll} 
C332 & $4487(1)$ & $1657(2)$ & $5298(1)$ & $17(1)$ \\
C342 & $4554(1)$ & $1065(2)$ & $4869(1)$ & $21(1)$ \\
C352 & $4966(1)$ & $1341(2)$ & $4594(1)$ & $22(1)$ \\
C362 & $5313(1)$ & $2207(2)$ & $4751(1)$ & $23(1)$ \\
C372 & $5244(1)$ & $2817(2)$ & $5180(1)$ & $18(1)$ \\
C382 & $5233(1)$ & $4295(2)$ & $6148(1)$ & $14(1)$ \\
C392 & $5571(1)$ & $4072(2)$ & $6550(1)$ & $18(1)$ \\
C402 & $6010(1)$ & $4767(2)$ & $6642(1)$ & $25(1)$ \\
C412 & $6106(1)$ & $5688(2)$ & $6338(1)$ & $27(1)$ \\
C422 & $5765(1)$ & $5938(2)$ & $5943(1)$ & $26(1)$ \\
C432 & $5331(1)$ & $5249(2)$ & $5846(1)$ & $23(1)$ \\
C442 & $4166(1)$ & $4355(2)$ & $5809(1)$ & $14(1)$ \\
C452 & $4062(1)$ & $5313(2)$ & $6102(1)$ & $17(1)$ \\
C462 & $3660(1)$ & $6082(2)$ & $5973(1)$ & $22(1)$ \\
C472 & $3354(1)$ & $5902(2)$ & $5547(1)$ & $26(1)$ \\
C482 & $3450(1)$ & $4952(3)$ & $5256(1)$ & $30(1)$ \\
C492 & $3853(1)$ & $4180(2)$ & $5382(1)$ & $23(1)$ \\
\hline
\end{tabular}

Table S25. Anisotropic displacement parameters $\left(\AA^{2} \times 10^{3}\right)$ for palladium complex 3. The anisotropic displacement factor exponent takes the form: $-2 \pi^{2}\left[\mathrm{~h}^{2} \mathrm{a}^{\cdot 2} \mathrm{U}^{11}+\ldots+2 \mathrm{~h} \mathrm{ka} \cdot \mathrm{b} \cdot \mathrm{U}^{12}\right]$.

\begin{tabular}{ccccccc}
\hline & $\mathrm{U}^{11}$ & $\mathrm{U}^{22}$ & $\mathrm{U}^{22}$ & $\mathrm{U}^{23}$ & $\mathrm{U}^{13}$ & $\mathrm{U}^{12}$ \\
\hline $\mathrm{Pd} 11$ & $14(1)$ & $13(1)$ & $11(1)$ & $-3(1)$ & $3(1)$ & $-2(1)$ \\
$\mathrm{S} 11$ & $23(1)$ & $17(1)$ & $16(1)$ & $-1(1)$ & $2(1)$ & $6(1)$ \\
$\mathrm{P} 11$ & $14(1)$ & $11(1)$ & $11(1)$ & $0(1)$ & $2(1)$ & $-2(1)$ \\
P21 & $15(1)$ & $16(1)$ & $16(1)$ & $-5(1)$ & $4(1)$ & $-1(1)$ \\
$\mathrm{S} 111$ & $13(1)$ & $11(1)$ & $11(1)$ & $0(1)$ & $1(1)$ & $0(1)$ \\
$\mathrm{C} 11$ & $12(1)$ & $12(1)$ & $12(1)$ & $-2(1)$ & $1(1)$ & $-2(1)$ \\
C21 & $15(1)$ & $15(1)$ & $12(1)$ & $-2(1)$ & $2(1)$ & $-4(1)$ \\
C31 & $25(2)$ & $28(2)$ & $17(1)$ & $7(1)$ & $4(1)$ & $4(1)$ \\
C41 & $18(1)$ & $44(2)$ & $24(2)$ & $5(1)$ & $4(1)$ & $5(1)$ \\
C51 & $22(2)$ & $39(2)$ & $14(1)$ & $-2(1)$ & $4(1)$ & $-14(1)$ \\
C61 & $35(2)$ & $19(1)$ & $21(1)$ & $6(1)$ & $4(1)$ & $-9(1)$ \\
C71 & $24(2)$ & $19(1)$ & $19(1)$ & $2(1)$ & $3(1)$ & $-2(1)$ \\
C81 & $21(1)$ & $20(1)$ & $8(1)$ & $1(1)$ & $3(1)$ & $-8(1)$ \\
C91 & $24(2)$ & $25(2)$ & $19(1)$ & $-4(1)$ & $7(1)$ & $-4(1)$ \\
C101 & $39(2)$ & $28(2)$ & $24(2)$ & $-9(1)$ & $8(1)$ & $-13(1)$ \\
C111 & $42(2)$ & $35(2)$ & $18(2)$ & $1(1)$ & $-8(1)$ & $-21(1)$ \\
C121 & $29(2)$ & $35(2)$ & $24(2)$ & $10(1)$ & $-12(1)$ & $-10(1)$ \\
C131 & $23(2)$ & $24(2)$ & $22(1)$ & $5(1)$ & $-5(1)$ & $-3(1)$
\end{tabular}




\begin{tabular}{|c|c|c|c|c|c|c|}
\hline C141 & $22(1)$ & $14(1)$ & $9(1)$ & 1(1) & $2(1)$ & $2(1)$ \\
\hline C151 & $23(1)$ & $20(1)$ & $18(1)$ & $-2(1)$ & 1(1) & $4(1)$ \\
\hline C161 & $28(2)$ & $31(2)$ & $21(2)$ & $-2(1)$ & $5(1)$ & $13(1)$ \\
\hline C171 & $50(2)$ & $19(2)$ & $17(1)$ & $-1(1)$ & $6(1)$ & $13(1)$ \\
\hline C181 & $45(2)$ & $16(1)$ & $26(2)$ & $-5(1)$ & $2(1)$ & $-1(1)$ \\
\hline C191 & $28(2)$ & $19(1)$ & $25(2)$ & $-1(1)$ & $2(1)$ & $1(1)$ \\
\hline C201 & $15(1)$ & $9(1)$ & $19(1)$ & $3(1)$ & $1(1)$ & $-2(1)$ \\
\hline C211 & $18(1)$ & $31(2)$ & $20(1)$ & $-3(1)$ & $2(1)$ & $-1(1)$ \\
\hline $\mathrm{C} 221$ & $21(2)$ & $43(2)$ & $28(2)$ & $-5(1)$ & $10(1)$ & $0(1)$ \\
\hline $\mathrm{C} 231$ & $13(1)$ & $21(1)$ & $40(2)$ & $0(1)$ & $4(1)$ & $1(1)$ \\
\hline C241 & $17(1)$ & $18(1)$ & $26(1)$ & $5(1)$ & $-5(1)$ & $-2(1)$ \\
\hline C251 & $18(1)$ & $19(1)$ & $18(1)$ & $3(1)$ & $2(1)$ & $-3(1)$ \\
\hline C261 & 11(1) & $20(1)$ & $13(1)$ & $0(1)$ & $3(1)$ & $0(1)$ \\
\hline $\mathrm{C} 271$ & $18(1)$ & $21(1)$ & $12(1)$ & 1(1) & $4(1)$ & $-2(1)$ \\
\hline C281 & $43(2)$ & $25(2)$ & $16(1)$ & $-2(1)$ & $-2(1)$ & $-11(1)$ \\
\hline C291 & $38(2)$ & $41(2)$ & $11(1)$ & $-1(1)$ & $-5(1)$ & $-8(1)$ \\
\hline C301 & $24(2)$ & $33(2)$ & $12(1)$ & $7(1)$ & $0(1)$ & $2(1)$ \\
\hline C311 & $17(1)$ & $20(1)$ & $17(1)$ & $2(1)$ & $4(1)$ & $3(1)$ \\
\hline C321 & $18(1)$ & $14(1)$ & $17(1)$ & $-1(1)$ & $2(1)$ & $0(1)$ \\
\hline C331 & $30(2)$ & $28(2)$ & $20(1)$ & $-4(1)$ & $5(1)$ & $-4(1)$ \\
\hline C341 & $34(2)$ & $32(2)$ & $20(2)$ & $-5(1)$ & $-5(1)$ & $-1(1)$ \\
\hline C351 & $21(2)$ & $25(2)$ & $32(2)$ & $0(1)$ & $-10(1)$ & $-5(1)$ \\
\hline C361 & $25(2)$ & $32(2)$ & $30(2)$ & $-1(1)$ & $5(1)$ & $-9(1)$ \\
\hline C371 & $26(2)$ & $34(2)$ & $19(1)$ & $-6(1)$ & $6(1)$ & $-7(1)$ \\
\hline C381 & $18(1)$ & $22(1)$ & $15(1)$ & $-5(1)$ & $5(1)$ & $0(1)$ \\
\hline C391 & $30(2)$ & $28(2)$ & $42(2)$ & $2(1)$ & $-7(1)$ & $-5(1)$ \\
\hline $\mathrm{C} 401$ & $26(2)$ & $47(2)$ & $39(2)$ & $-2(2)$ & $-12(1)$ & $-3(1)$ \\
\hline C411 & $26(2)$ & $38(2)$ & $26(2)$ & $-2(1)$ & $3(1)$ & $14(1)$ \\
\hline C421 & $32(2)$ & $25(2)$ & $58(2)$ & $2(1)$ & $11(2)$ & $8(1)$ \\
\hline $\mathrm{C} 431$ & $21(2)$ & $25(2)$ & $45(2)$ & $-5(1)$ & $4(1)$ & $1(1)$ \\
\hline C441 & $24(2)$ & $21(1)$ & $25(2)$ & $-5(1)$ & $10(1)$ & $-4(1)$ \\
\hline $\mathrm{C} 45 \mathrm{~B} 1$ & $23(6)$ & $31(8)$ & $26(5)$ & $13(6)$ & $5(4)$ & $9(5)$ \\
\hline $\mathrm{C} 451$ & $48(7)$ & $23(5)$ & $43(5)$ & $0(4)$ & $28(4)$ & $-3(4)$ \\
\hline C461 & $33(2)$ & $72(3)$ & $25(2)$ & $-5(2)$ & 11(1) & $13(2)$ \\
\hline C471 & $36(4)$ & $47(3)$ & $33(4)$ & $9(3)$ & $21(2)$ & 1(3) \\
\hline C481 & $59(2)$ & $56(2)$ & $59(2)$ & $2(1)$ & $9(1)$ & $0(1)$ \\
\hline C491 & $44(3)$ & $24(2)$ & $52(3)$ & $3(2)$ & $31(2)$ & $1(2)$ \\
\hline C47B1 & $36(4)$ & $47(3)$ & $33(4)$ & $9(3)$ & $21(2)$ & 1(3) \\
\hline C48B1 & $50(5)$ & $32(4)$ & $36(4)$ & $20(3)$ & $20(4)$ & $4(3)$ \\
\hline
\end{tabular}




\begin{tabular}{|c|c|c|c|c|c|c|}
\hline C49B1 & $44(3)$ & $24(2)$ & $52(3)$ & $3(2)$ & $31(2)$ & $1(2)$ \\
\hline $\mathrm{Pd} 12$ & $11(1)$ & $9(1)$ & $9(1)$ & $2(1)$ & $3(1)$ & $1(1)$ \\
\hline S22 & $15(1)$ & $13(1)$ & $13(1)$ & $3(1)$ & $4(1)$ & $4(1)$ \\
\hline $\mathrm{P} 12$ & $13(1)$ & $11(1)$ & $10(1)$ & $1(1)$ & $3(1)$ & $1(1)$ \\
\hline $\mathrm{P} 22$ & $13(1)$ & $11(1)$ & $10(1)$ & $2(1)$ & $2(1)$ & $0(1)$ \\
\hline Si12 & $13(1)$ & $10(1)$ & $12(1)$ & $1(1)$ & $3(1)$ & $1(1)$ \\
\hline $\mathrm{C} 12$ & $13(1)$ & $12(1)$ & $12(1)$ & $2(1)$ & $2(1)$ & $2(1)$ \\
\hline $\mathrm{C} 22$ & $17(1)$ & $15(1)$ & $15(1)$ & $-3(1)$ & $7(1)$ & $-3(1)$ \\
\hline C32 & $20(1)$ & $25(2)$ & $24(2)$ & $0(1)$ & $3(1)$ & $-3(1)$ \\
\hline $\mathrm{C} 42$ & $17(2)$ & $41(2)$ & $36(2)$ & $1(1)$ & $2(1)$ & $-4(1)$ \\
\hline C52 & $25(2)$ & $38(2)$ & $47(2)$ & $0(2)$ & $14(1)$ & $-12(1)$ \\
\hline C62 & $38(2)$ & $24(2)$ & $35(2)$ & $6(1)$ & $16(1)$ & $-9(1)$ \\
\hline $\mathrm{C} 72$ & $25(2)$ & $21(2)$ & $30(2)$ & $6(1)$ & $10(1)$ & $1(1)$ \\
\hline $\mathrm{C} 82$ & $21(1)$ & $12(1)$ & $10(1)$ & $2(1)$ & $1(1)$ & $4(1)$ \\
\hline $\mathrm{C} 92$ & $20(1)$ & $29(2)$ & $17(1)$ & $-3(1)$ & $2(1)$ & $5(1)$ \\
\hline C102 & $31(2)$ & $31(2)$ & $17(1)$ & $-2(1)$ & $7(1)$ & $8(1)$ \\
\hline C112 & $37(2)$ & $21(1)$ & $12(1)$ & $-1(1)$ & $-4(1)$ & $5(1)$ \\
\hline C122 & $28(2)$ & $19(1)$ & $21(1)$ & $1(1)$ & $0(1)$ & $-1(1)$ \\
\hline C132 & $23(1)$ & $14(1)$ & $14(1)$ & $-1(1)$ & $6(1)$ & $-2(1)$ \\
\hline C142 & $11(1)$ & $12(1)$ & $16(1)$ & $2(1)$ & $3(1)$ & $1(1)$ \\
\hline C152 & $17(1)$ & $16(1)$ & $21(1)$ & $0(1)$ & $0(1)$ & $3(1)$ \\
\hline C162 & $20(1)$ & 11(1) & $44(2)$ & $5(1)$ & $0(1)$ & $-1(1)$ \\
\hline C172 & $22(2)$ & $28(2)$ & $40(2)$ & $23(1)$ & $4(1)$ & $4(1)$ \\
\hline C182 & $31(2)$ & $35(2)$ & $19(1)$ & $10(1)$ & $3(1)$ & $7(1)$ \\
\hline C192 & $24(1)$ & $18(1)$ & $19(1)$ & $2(1)$ & $1(1)$ & $3(1)$ \\
\hline C202 & $17(1)$ & $10(1)$ & $18(1)$ & $1(1)$ & $1(1)$ & $2(1)$ \\
\hline C212 & $24(1)$ & $17(1)$ & $20(1)$ & $1(1)$ & $2(1)$ & $0(1)$ \\
\hline C222 & $39(2)$ & $22(2)$ & $18(1)$ & $0(1)$ & $-6(1)$ & $4(1)$ \\
\hline C232 & $29(2)$ & $23(2)$ & $36(2)$ & $-3(1)$ & $-15(1)$ & $4(1)$ \\
\hline C242 & $17(2)$ & $34(2)$ & $45(2)$ & $1(1)$ & $-2(1)$ & $-3(1)$ \\
\hline C252 & $20(1)$ & $24(2)$ & $25(2)$ & $2(1)$ & $2(1)$ & $1(1)$ \\
\hline C262 & $15(1)$ & $14(1)$ & 11(1) & $-2(1)$ & $2(1)$ & $2(1)$ \\
\hline C272 & $21(1)$ & $15(1)$ & $18(1)$ & $1(1)$ & $5(1)$ & $2(1)$ \\
\hline C282 & $18(1)$ & $20(1)$ & $23(1)$ & $0(1)$ & $7(1)$ & $7(1)$ \\
\hline C292 & $13(1)$ & $22(1)$ & $19(1)$ & $0(1)$ & $7(1)$ & $1(1)$ \\
\hline C302 & $19(1)$ & $14(1)$ & $10(1)$ & $0(1)$ & $2(1)$ & $-1(1)$ \\
\hline C312 & $15(1)$ & $14(1)$ & $7(1)$ & $-1(1)$ & $-1(1)$ & $0(1)$ \\
\hline C322 & $17(1)$ & $14(1)$ & $9(1)$ & $3(1)$ & $1(1)$ & $5(1)$ \\
\hline C332 & $17(1)$ & $15(1)$ & $20(1)$ & $4(1)$ & $1(1)$ & $1(1)$ \\
\hline
\end{tabular}




\begin{tabular}{lllllll}
$\mathrm{C} 342$ & $25(2)$ & $17(1)$ & $20(1)$ & $-1(1)$ & $-3(1)$ & $6(1)$ \\
$\mathrm{C} 352$ & $32(2)$ & $24(2)$ & $11(1)$ & $-1(1)$ & $2(1)$ & $12(1)$ \\
$\mathrm{C} 362$ & $23(2)$ & $32(2)$ & $15(1)$ & $7(1)$ & $9(1)$ & $8(1)$ \\
$\mathrm{C} 372$ & $19(1)$ & $20(1)$ & $15(1)$ & $6(1)$ & $0(1)$ & $2(1)$ \\
$\mathrm{C} 382$ & $16(1)$ & $12(1)$ & $16(1)$ & $-1(1)$ & $4(1)$ & $0(1)$ \\
$\mathrm{C} 392$ & $22(1)$ & $15(1)$ & $15(1)$ & $0(1)$ & $1(1)$ & $0(1)$ \\
$\mathrm{C} 402$ & $25(2)$ & $26(2)$ & $22(1)$ & $-5(1)$ & $-5(1)$ & $-1(1)$ \\
$\mathrm{C} 412$ & $26(2)$ & $22(2)$ & $34(2)$ & $-5(1)$ & $4(1)$ & $-9(1)$ \\
$\mathrm{C} 422$ & $31(2)$ & $20(2)$ & $29(2)$ & $7(1)$ & $4(1)$ & $-8(1)$ \\
$\mathrm{C} 432$ & $26(2)$ & $20(1)$ & $21(1)$ & $5(1)$ & $1(1)$ & $-2(1)$ \\
$\mathrm{C} 442$ & $15(1)$ & $16(1)$ & $13(1)$ & $7(1)$ & $2(1)$ & $-1(1)$ \\
$\mathrm{C} 452$ & $21(1)$ & $16(1)$ & $14(1)$ & $5(1)$ & $1(1)$ & $0(1)$ \\
$\mathrm{C} 462$ & $25(2)$ & $15(1)$ & $26(2)$ & $3(1)$ & $4(1)$ & $3(1)$ \\
$\mathrm{C} 472$ & $21(2)$ & $23(2)$ & $34(2)$ & $7(1)$ & $-3(1)$ & $7(1)$ \\
$\mathrm{C} 482$ & $28(2)$ & $31(2)$ & $29(2)$ & $1(1)$ & $-13(1)$ & $7(1)$ \\
$\mathrm{C} 492$ & $23(2)$ & $22(1)$ & $22(1)$ & $-2(1)$ & $-4(1)$ & $5(1)$ \\
\hline
\end{tabular}

\subsection{Crystal Structure Determination of 5}

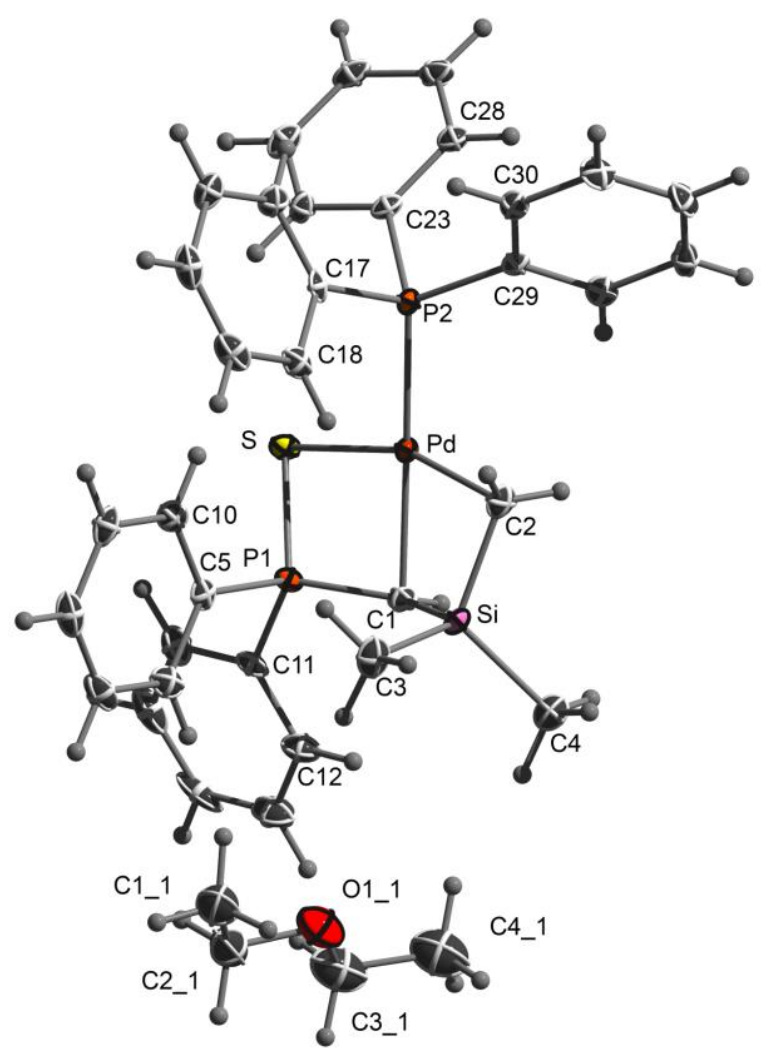

Figure S55. ORTEP Plot of palladium complex 5. Ellipsoids are drawn at the 50\% probability level 
Table S26. Atomic coordinates $\left(\mathrm{x} 10^{4}\right)$ and equivalent isotropic displacement parameters $\left(\AA^{2} \times 10^{3}\right)$ for palladium complex $\mathbf{5}$. U(eq) is defined as one third of the trace of the orthogonalized $\mathrm{U}^{\mathrm{ij}}$ tensor.

\begin{tabular}{|c|c|c|c|c|}
\hline & $\mathrm{X}$ & $\mathrm{y}$ & $\mathrm{Z}$ & $\mathrm{U}(\mathrm{eq})$ \\
\hline $\operatorname{Pd}(1)$ & $8272(1)$ & $7043(1)$ & $6557(1)$ & $10(1)$ \\
\hline $\mathrm{S}(1)$ & $6027(1)$ & $6755(1)$ & $7121(1)$ & $13(1)$ \\
\hline $\mathrm{P}(1)$ & $6702(1)$ & $7306(1)$ & $8162(1)$ & $11(1)$ \\
\hline $\operatorname{Si}(1)$ & $9776(1)$ & $7553(1)$ & $7789(1)$ & $14(1)$ \\
\hline $\mathrm{C}(1)$ & $8359(3)$ & $6833(2)$ & $8046(2)$ & $14(1)$ \\
\hline $\mathrm{P}(2)$ & $8321(1)$ & $7528(1)$ & $4985(1)$ & 11(1) \\
\hline $\mathrm{C}(2)$ & $10139(3)$ & $7319(3)$ & $6562(2)$ & $20(1)$ \\
\hline $\mathrm{C}(3)$ & $9428(3)$ & $9131(2)$ & $7916(2)$ & $23(1)$ \\
\hline $\mathrm{C}(4)$ & $11067(3)$ & $6794(3)$ & $8528(2)$ & $25(1)$ \\
\hline$C(5)$ & $6271(2)$ & $8881(2)$ & $8092(2)$ & $12(1)$ \\
\hline$C(6)$ & $6177(3)$ & $9433(2)$ & $8883(2)$ & $18(1)$ \\
\hline $\mathrm{C}(7)$ & $5833(3)$ & $10634(2)$ & $8826(2)$ & $21(1)$ \\
\hline $\mathrm{C}(8)$ & $5584(3)$ & $11297(2)$ & $7988(2)$ & $21(1)$ \\
\hline $\mathrm{C}(9)$ & $5690(3)$ & $10766(2)$ & $7198(2)$ & $20(1)$ \\
\hline$C(10)$ & $6029(2)$ & $9560(2)$ & $7253(2)$ & $15(1)$ \\
\hline $\mathrm{C}(11)$ & $5926(3)$ & $6787(2)$ & $9285(2)$ & $14(1)$ \\
\hline $\mathrm{C}(12)$ & $6644(3)$ & $6367(2)$ & $10021(2)$ & $22(1)$ \\
\hline $\mathrm{C}(13)$ & $6054(3)$ & $5954(3)$ & $10875(2)$ & $29(1)$ \\
\hline $\mathrm{C}(14)$ & $4755(3)$ & $5952(3)$ & 10992(2) & $27(1)$ \\
\hline$C(15)$ & $4029(3)$ & $6388(3)$ & $10271(2)$ & $26(1)$ \\
\hline$C(16)$ & $4612(3)$ & $6800(2)$ & $9414(2)$ & $21(1)$ \\
\hline$C(17)$ & $7741(2)$ & $9088(2)$ & $4660(2)$ & $12(1)$ \\
\hline $\mathrm{C}(18)$ & $8027(2)$ & $9868(2)$ & $5194(2)$ & $16(1)$ \\
\hline$C(19)$ & $7671(3)$ & $11064(2)$ & $4942(2)$ & $19(1)$ \\
\hline $\mathrm{C}(20)$ & $7043(3)$ & $11492(2)$ & $4171(2)$ & $19(1)$ \\
\hline $\mathrm{C}(21)$ & $6748(3)$ & $10724(2)$ & $3644(2)$ & $19(1)$ \\
\hline$C(22)$ & $7095(2)$ & $9530(2)$ & $3889(2)$ & $16(1)$ \\
\hline $\mathrm{C}(23)$ & $7322(2)$ & $6822(2)$ & $4422(2)$ & $12(1)$ \\
\hline $\mathrm{C}(24)$ & $6002(2)$ & $6999(2)$ & $4708(2)$ & $14(1)$ \\
\hline $\mathrm{C}(25)$ & $5213(3)$ & $6420(2)$ & $4358(2)$ & $17(1)$ \\
\hline$C(26)$ & $5732(3)$ & $5647(2)$ & $3721(2)$ & $19(1)$ \\
\hline $\mathrm{C}(27)$ & $7043(3)$ & $5461(2)$ & $3437(2)$ & $17(1)$ \\
\hline $\mathrm{C}(28)$ & $7831(3)$ & $6056(2)$ & $3775(2)$ & $15(1)$ \\
\hline $\mathrm{C}(29)$ & $9885(2)$ & $7251(2)$ & $4286(2)$ & $12(1)$ \\
\hline $\mathrm{C}(30)$ & $10211(3)$ & $7986(2)$ & $3485(2)$ & $15(1)$ \\
\hline $\mathrm{C}(31)$ & $11378(3)$ & $7698(2)$ & $2949(2)$ & $19(1)$ \\
\hline
\end{tabular}




\begin{tabular}{rrrrr}
$\mathrm{C}(32)$ & $12226(3)$ & $6680(2)$ & $3200(2)$ & $19(1)$ \\
$\mathrm{C}(33)$ & $11926(3)$ & $5955(2)$ & $4000(2)$ & $18(1)$ \\
$\mathrm{C}(34)$ & $10758(2)$ & $6248(2)$ & $4543(2)$ & $14(1)$ \\
$\mathrm{O} 11$ & $8749(2)$ & $8637(2)$ & $10843(2)$ & $39(1)$ \\
$\mathrm{C} 11$ & $8258(3)$ & $10668(3)$ & $10349(3)$ & $43(1)$ \\
$\mathrm{C} 21$ & $7891(3)$ & $9677(3)$ & $11043(2)$ & $39(1)$ \\
$\mathrm{C} 31$ & $8524(4)$ & $7659(3)$ & $11502(2)$ & $44(1)$ \\
$\mathrm{C} 41$ & $9561(4)$ & $6627(3)$ & $11300(3)$ & $52(1)$ \\
\hline
\end{tabular}

Table S27. Anisotropic displacement parameters $\left(\AA^{2} \times 10^{3}\right)$ for palladium complex 5. The anisotropic displacement factor exponent takes the form: $-2 \pi^{2}\left[\mathrm{~h}^{2} \mathrm{a}^{\cdot 2} \mathrm{U}^{11}+\ldots+2 \mathrm{~h} \mathrm{ka} \cdot \mathrm{b} \cdot \mathrm{U}^{12}\right]$.

\begin{tabular}{|c|c|c|c|c|c|c|}
\hline & $\mathrm{U}^{11}$ & $\mathrm{U}^{22}$ & $\mathrm{U}^{22}$ & $\mathrm{U}^{23}$ & $\mathrm{U}^{13}$ & $\mathrm{U}^{12}$ \\
\hline $\operatorname{Pd}(1)$ & $9(1)$ & $12(1)$ & 11(1) & $-2(1)$ & $-1(1)$ & $-3(1)$ \\
\hline $\mathrm{S}(1)$ & $13(1)$ & $16(1)$ & $13(1)$ & $-4(1)$ & $-1(1)$ & $-6(1)$ \\
\hline $\mathrm{P}(1)$ & $13(1)$ & 11(1) & 11(1) & $-2(1)$ & $-1(1)$ & $-4(1)$ \\
\hline $\mathrm{Si}(1)$ & 11(1) & $17(1)$ & $16(1)$ & $-3(1)$ & $-3(1)$ & $-3(1)$ \\
\hline $\mathrm{C}(1)$ & $17(1)$ & $12(2)$ & 11(1) & $0(1)$ & $-2(1)$ & $-2(1)$ \\
\hline $\mathrm{P}(2)$ & $9(1)$ & $13(1)$ & $12(1)$ & $-2(1)$ & $-1(1)$ & $-3(1)$ \\
\hline $\mathrm{C}(2)$ & $9(1)$ & $30(2)$ & 21(2) & $-5(1)$ & $-2(1)$ & $-6(1)$ \\
\hline$C(3)$ & $16(1)$ & $20(2)$ & $33(2)$ & $-5(1)$ & $-3(1)$ & $-6(1)$ \\
\hline $\mathrm{C}(4)$ & $21(2)$ & $29(2)$ & $26(2)$ & $-4(1)$ & $-7(1)$ & $-1(1)$ \\
\hline$C(5)$ & $7(1)$ & $14(1)$ & $17(1)$ & $-3(1)$ & $1(1)$ & $-5(1)$ \\
\hline$C(6)$ & $17(1)$ & $20(2)$ & $16(2)$ & $-3(1)$ & $1(1)$ & $-7(1)$ \\
\hline$C(7)$ & $19(2)$ & $20(2)$ & $26(2)$ & $-12(1)$ & $3(1)$ & $-7(1)$ \\
\hline $\mathrm{C}(8)$ & $14(1)$ & $14(2)$ & $33(2)$ & $-6(1)$ & $4(1)$ & $-4(1)$ \\
\hline $\mathrm{C}(9)$ & $15(1)$ & $15(2)$ & $27(2)$ & $2(1)$ & $-3(1)$ & $-3(1)$ \\
\hline$C(10)$ & $14(1)$ & $16(2)$ & $17(1)$ & $-3(1)$ & $-1(1)$ & $-6(1)$ \\
\hline $\mathrm{C}(11)$ & $19(1)$ & $12(1)$ & 12(1) & $-4(1)$ & $1(1)$ & $-5(1)$ \\
\hline $\mathrm{C}(12)$ & $27(2)$ & $23(2)$ & $14(2)$ & $-2(1)$ & $-1(1)$ & $-7(1)$ \\
\hline $\mathrm{C}(13)$ & $42(2)$ & $25(2)$ & $17(2)$ & 1(1) & $-3(2)$ & $-5(2)$ \\
\hline $\mathrm{C}(14)$ & $46(2)$ & $18(2)$ & $16(2)$ & $-6(1)$ & $15(2)$ & $-12(2)$ \\
\hline$C(15)$ & $25(2)$ & $20(2)$ & $32(2)$ & $-10(1)$ & $13(2)$ & $-7(1)$ \\
\hline$C(16)$ & $20(2)$ & $17(2)$ & $23(2)$ & $-3(1)$ & $4(1)$ & $-4(1)$ \\
\hline $\mathrm{C}(17)$ & $7(1)$ & $14(1)$ & $15(1)$ & $-3(1)$ & $3(1)$ & $-1(1)$ \\
\hline $\mathrm{C}(18)$ & $14(1)$ & $18(2)$ & $17(1)$ & $-5(1)$ & $2(1)$ & $-6(1)$ \\
\hline $\mathrm{C}(19)$ & $20(2)$ & $16(2)$ & $24(2)$ & $-8(1)$ & $4(1)$ & $-9(1)$ \\
\hline$C(20)$ & $14(1)$ & $12(1)$ & $30(2)$ & $-3(1)$ & $4(1)$ & $-3(1)$ \\
\hline $\mathrm{C}(21)$ & $14(1)$ & $20(2)$ & $24(2)$ & $0(1)$ & $-5(1)$ & $-2(1)$ \\
\hline
\end{tabular}




\begin{tabular}{lllllll}
$\mathrm{C}(22)$ & $14(1)$ & $15(2)$ & $18(2)$ & $-3(1)$ & $-2(1)$ & $-4(1)$ \\
$\mathrm{C}(23)$ & $13(1)$ & $12(1)$ & $11(1)$ & $2(1)$ & $-4(1)$ & $-5(1)$ \\
$\mathrm{C}(24)$ & $14(1)$ & $12(1)$ & $17(1)$ & $-2(1)$ & $-2(1)$ & $-2(1)$ \\
$\mathrm{C}(25)$ & $14(1)$ & $17(2)$ & $21(2)$ & $3(1)$ & $-5(1)$ & $-7(1)$ \\
$\mathrm{C}(26)$ & $20(1)$ & $18(2)$ & $21(2)$ & $-1(1)$ & $-9(1)$ & $-8(1)$ \\
$\mathrm{C}(27)$ & $22(2)$ & $14(2)$ & $18(2)$ & $-4(1)$ & $-8(1)$ & $-3(1)$ \\
$\mathrm{C}(28)$ & $16(1)$ & $15(2)$ & $12(1)$ & $1(1)$ & $-4(1)$ & $-3(1)$ \\
$\mathrm{C}(29)$ & $9(1)$ & $15(1)$ & $14(1)$ & $-5(1)$ & $-2(1)$ & $-5(1)$ \\
$\mathrm{C}(30)$ & $14(1)$ & $15(2)$ & $16(1)$ & $-2(1)$ & $-3(1)$ & $-2(1)$ \\
$\mathrm{C}(31)$ & $16(1)$ & $25(2)$ & $14(1)$ & $0(1)$ & $4(1)$ & $-9(1)$ \\
$\mathrm{C}(32)$ & $13(1)$ & $23(2)$ & $21(2)$ & $-7(1)$ & $5(1)$ & $-3(1)$ \\
$\mathrm{C}(33)$ & $15(1)$ & $15(2)$ & $25(2)$ & $-7(1)$ & $-2(1)$ & $-1(1)$ \\
$\mathrm{C}(34)$ & $14(1)$ & $14(1)$ & $15(1)$ & $-2(1)$ & $-1(1)$ & $-6(1)$ \\
$\mathrm{O} 11$ & $40(1)$ & $43(2)$ & $35(1)$ & $-8(1)$ & $5(1)$ & $-12(1)$ \\
$\mathrm{C} 11$ & $40(2)$ & $46(2)$ & $45(2)$ & $-18(2)$ & $-10(2)$ & $0(2)$ \\
$\mathrm{C} 21$ & $25(2)$ & $55(2)$ & $43(2)$ & $-25(2)$ & $-3(2)$ & $-6(2)$ \\
$\mathrm{C} 31$ & $48(2)$ & $53(3)$ & $36(2)$ & $-11(2)$ & $4(2)$ & $-27(2)$ \\
$\mathrm{C} 41$ & $68(3)$ & $48(3)$ & $43(2)$ & $-8(2)$ & $0(2)$ & $-20(2)$ \\
\hline
\end{tabular}

\section{Transition states for the formation of 6}

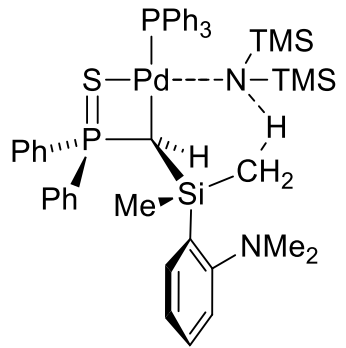

Transition state for the formation of $\left(R_{C}, S_{S i}\right)-6$

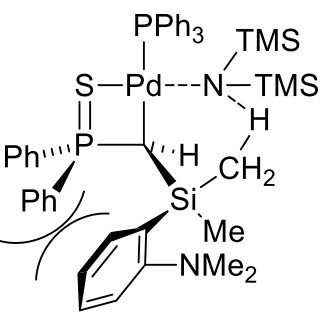

Transition state for the formation of $\left(R_{C}, R_{S i}\right)-6$

Figure S56. Possible diastereotopic transition states for the formation of $\mathbf{6}$. 


\section{High resolution Mass Spectra}

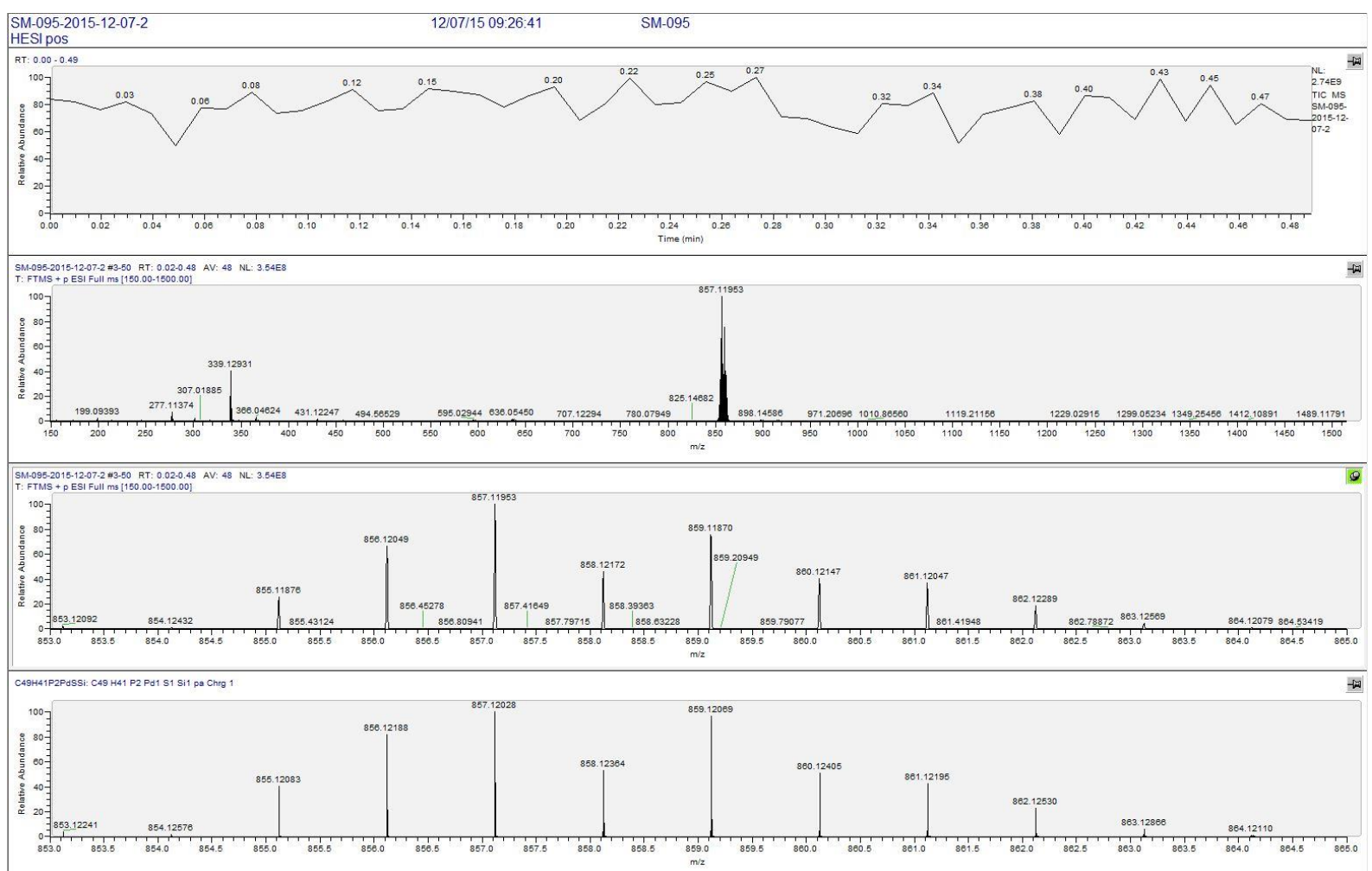

Figure S57. HRMS (ESI) spectrum of 2a; Calc. for $\mathrm{C}_{49} \mathrm{H}_{41} \mathrm{P}_{2} \mathrm{PdSSi}\left([\mathrm{M}-\mathrm{Cl}]^{+\cdot}\right): \mathrm{m} / \mathrm{z}=857.12028$; found: $\mathrm{m} / \mathrm{z}=$ 857.11951; observed and calculated peak profile.

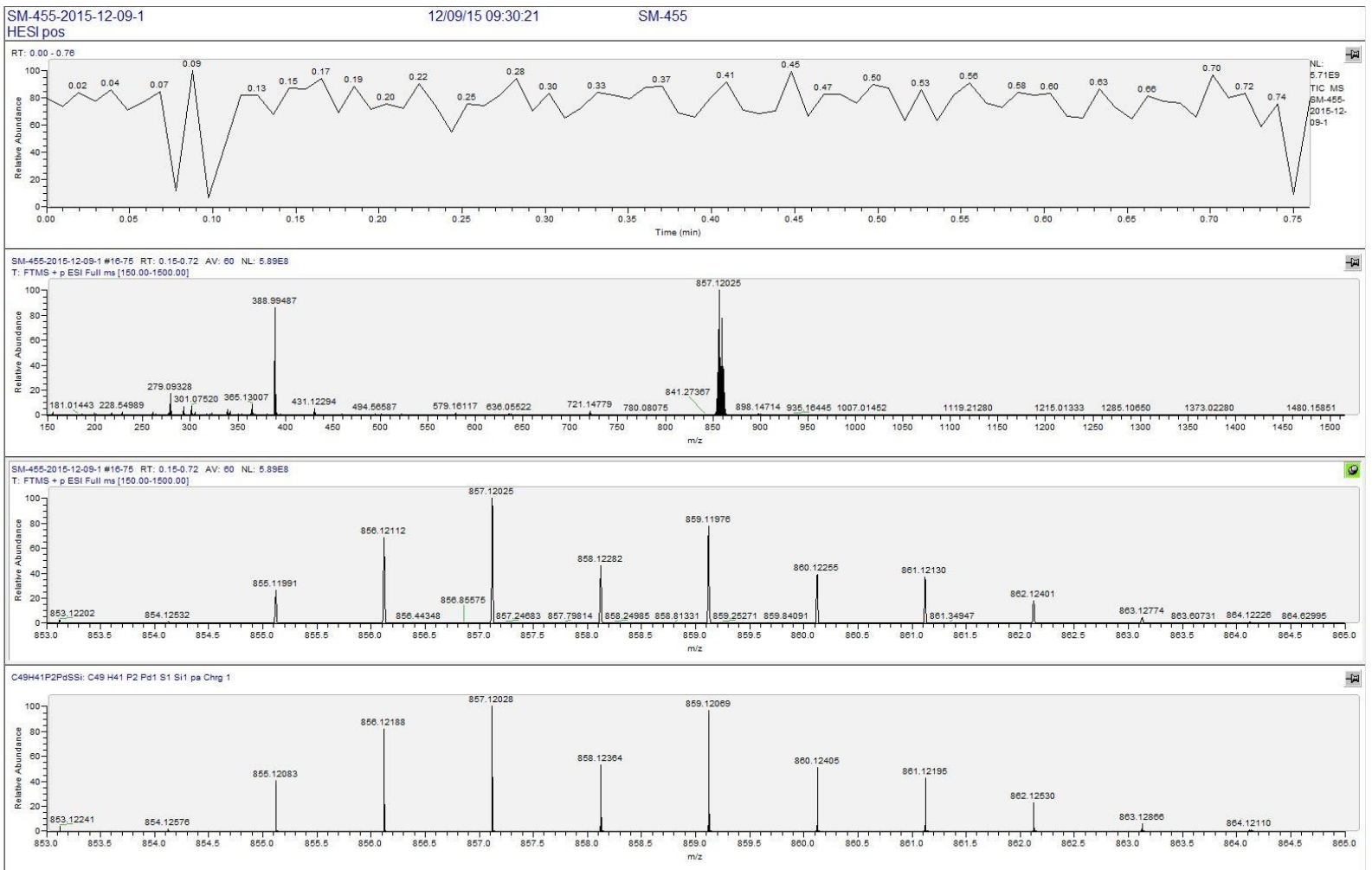

Figure S58. HRMS (ESI) spectrum of 2b. Calc. for $\mathrm{C}_{49} \mathrm{H}_{41} \mathrm{P}_{2} \mathrm{PdSSi}\left([\mathrm{M}-\mathrm{I}]^{+\cdot}\right): \mathrm{m} / \mathrm{z}=857.12028$; found: $\mathrm{m} / \mathrm{z}=$ 857.12025; observed and calculated peak profile. 


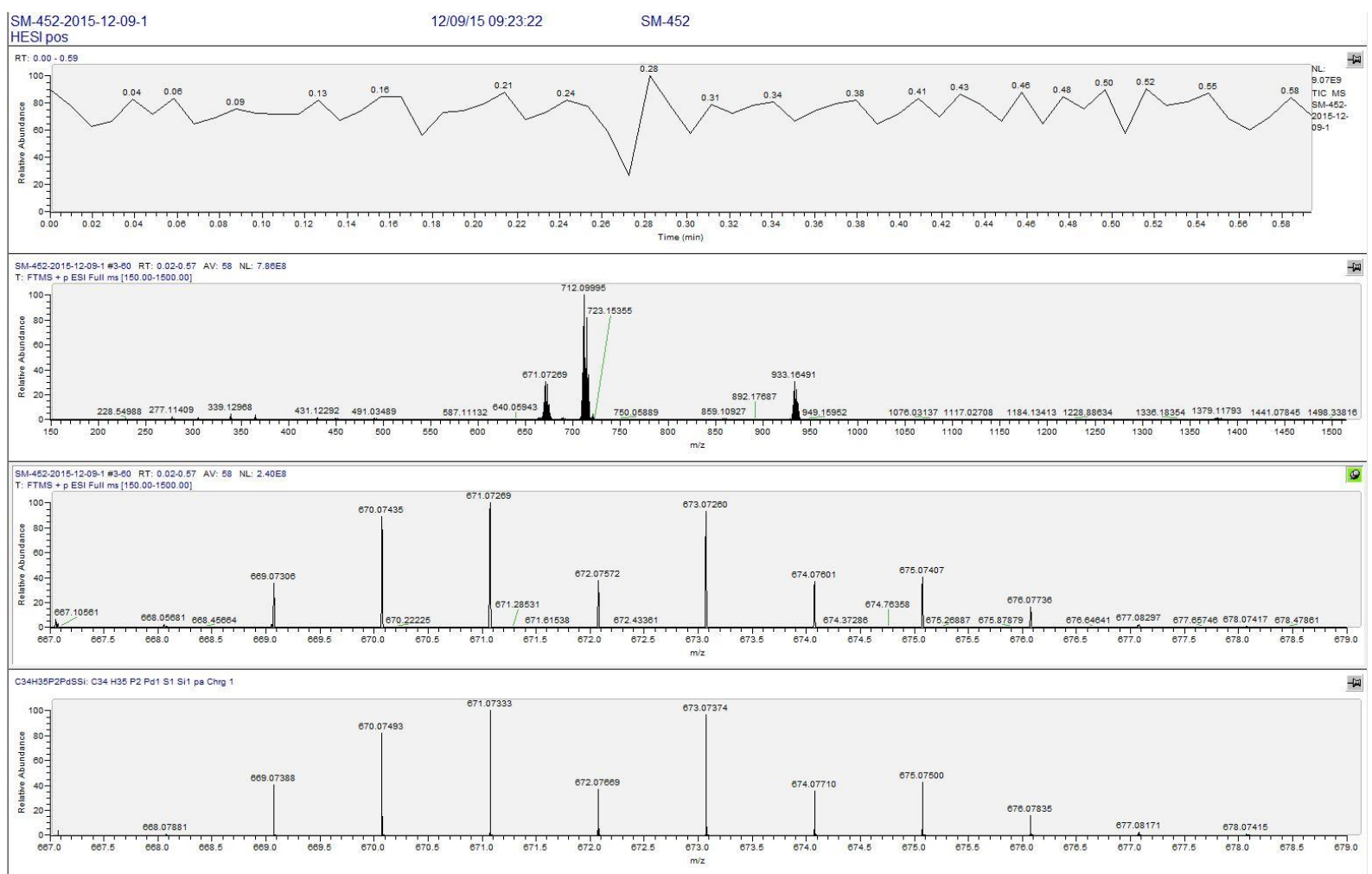

Figure S59. HRMS (ESI) spectrum of 2c. Calc. for $\mathrm{C}_{34} \mathrm{H}_{35} \mathrm{P}_{2} \mathrm{PdSSi}\left([\mathrm{M}-\mathrm{Cl}]^{+\cdot}\right): \mathrm{m} / \mathrm{z}=671.07333$; found: $\mathrm{m} / \mathrm{z}=$ 671.07270; observed and calculated peak profile.

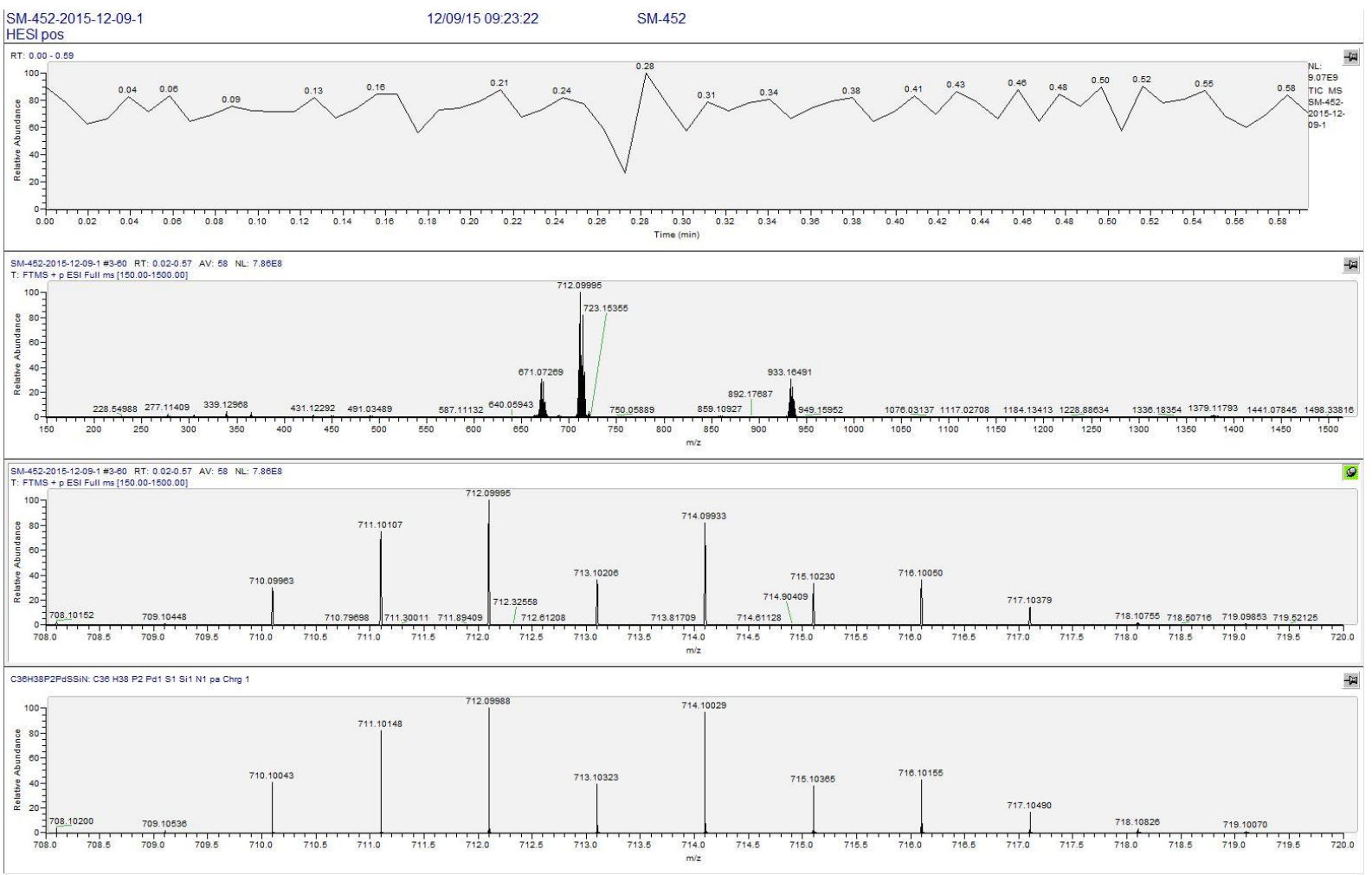

Figure S60. HRMS (ESI) spectrum of 2c. Calc. for $\mathrm{C}_{36} \mathrm{H}_{38} \mathrm{NP}_{2} \mathrm{PdSSi}\left(\left[\mathrm{M}-\mathrm{Cl}+\mathrm{H}_{3} \mathrm{CCN}\right]^{+\cdot}\right): \mathrm{m} / \mathrm{z}=712.09988$; found: $\mathrm{m} / \mathrm{z}=712.09995$; observed and calculated peak profile. 


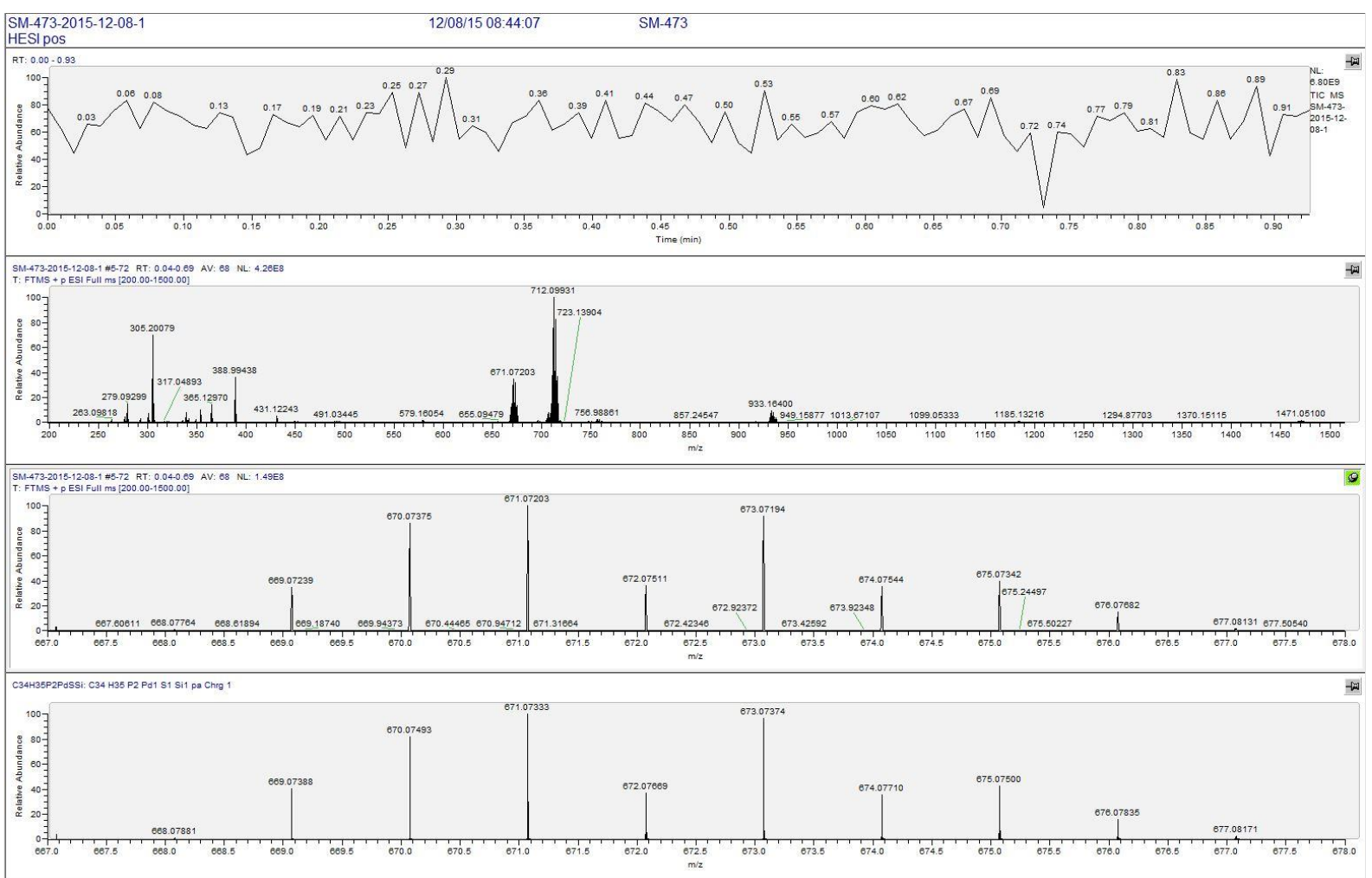

Figure S61. HRMS (ESI) spectrum of 2 d. Calc. for $\mathrm{C}_{34} \mathrm{H}_{35} \mathrm{P}{ }_{2} \mathrm{PdSSi}\left([\mathrm{M}-\mathrm{I}]^{+}\right.$): $\mathrm{m} / \mathrm{z}=671.07333$; found: $\mathrm{m} / \mathrm{z}=$ 671.07203; observed and calculated peak profile.

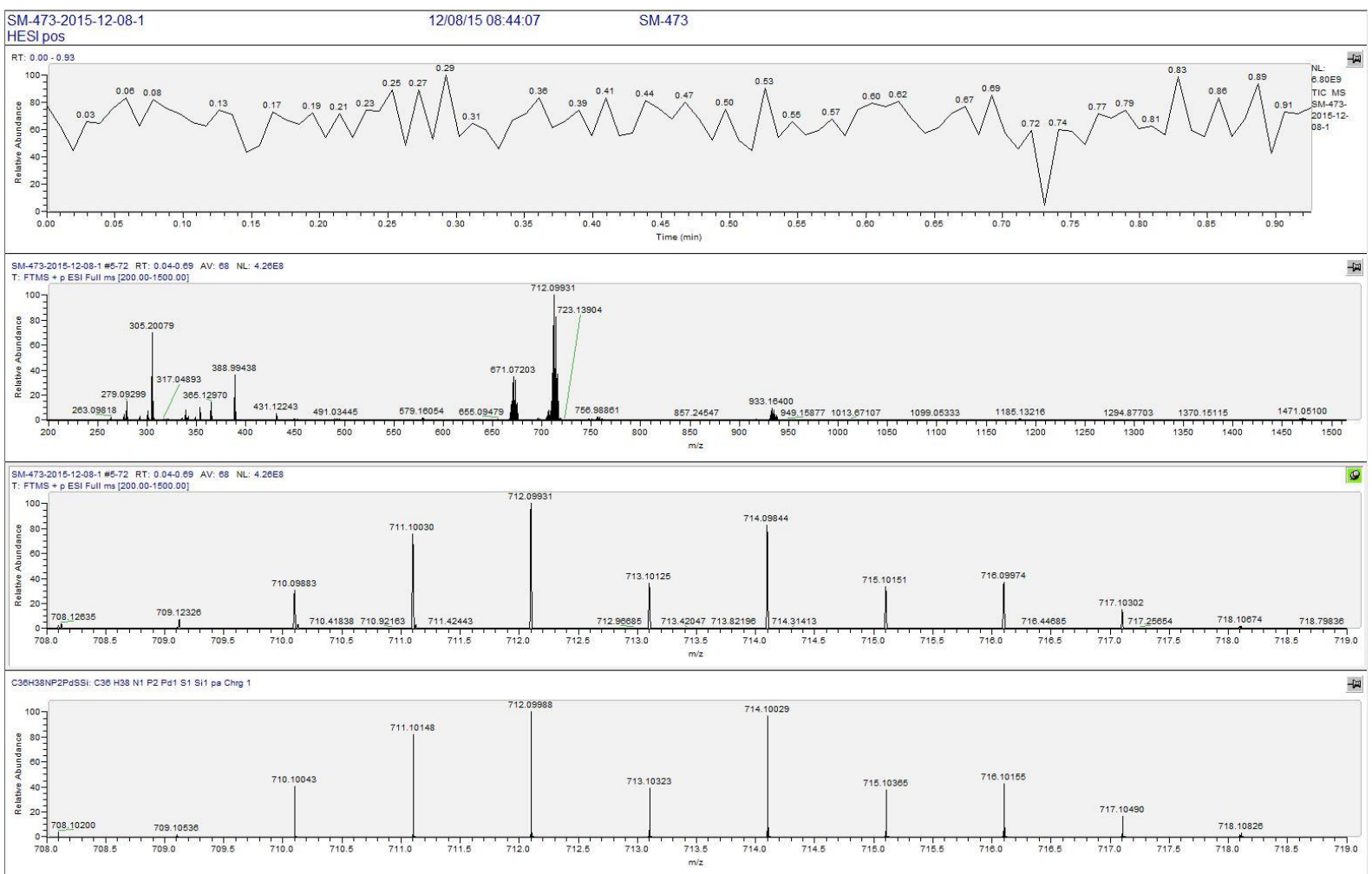

Figure S62. HRMS (ESI) spectrum of 2d. Calc. for $\mathrm{C}_{36} \mathrm{H}_{38} \mathrm{NP}_{2} \mathrm{PdSSi}\left(\left[\mathrm{M}-\mathrm{I}+\mathrm{H}_{3} \mathrm{CCN}\right]^{+}\right)$: $\mathrm{m} / \mathrm{z}=712.09988$; found: $\mathrm{m} / \mathrm{z}=712.09932$; observed and calculated peak profile. 


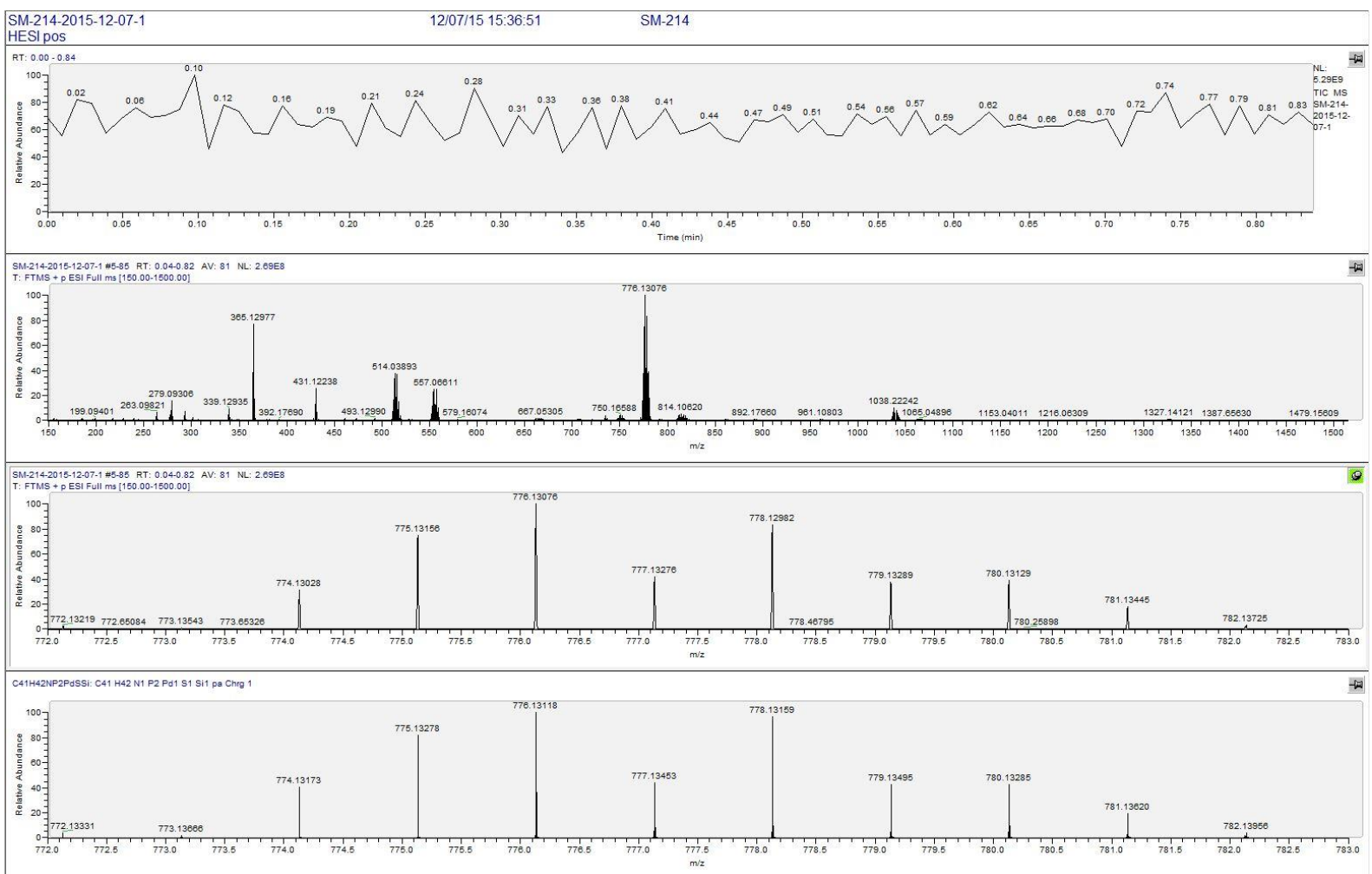

Figure S63. HRMS (ESI) spectrum of 2e. Calc. for $\mathrm{C}_{41} \mathrm{H}_{42} \mathrm{NP}_{2} \mathrm{PdSSi}\left([\mathrm{M}-\mathrm{Cl}]^{+}\right)$: $\mathrm{m} / \mathrm{z}=776.13118$; found: $\mathrm{m} / \mathrm{z}=$ 776.13077, $514.03893\left(\left[\mathrm{M}-\mathrm{Cl}-\mathrm{PPh}_{3}\right]^{+}\right)$; observed and calculated peak profile.

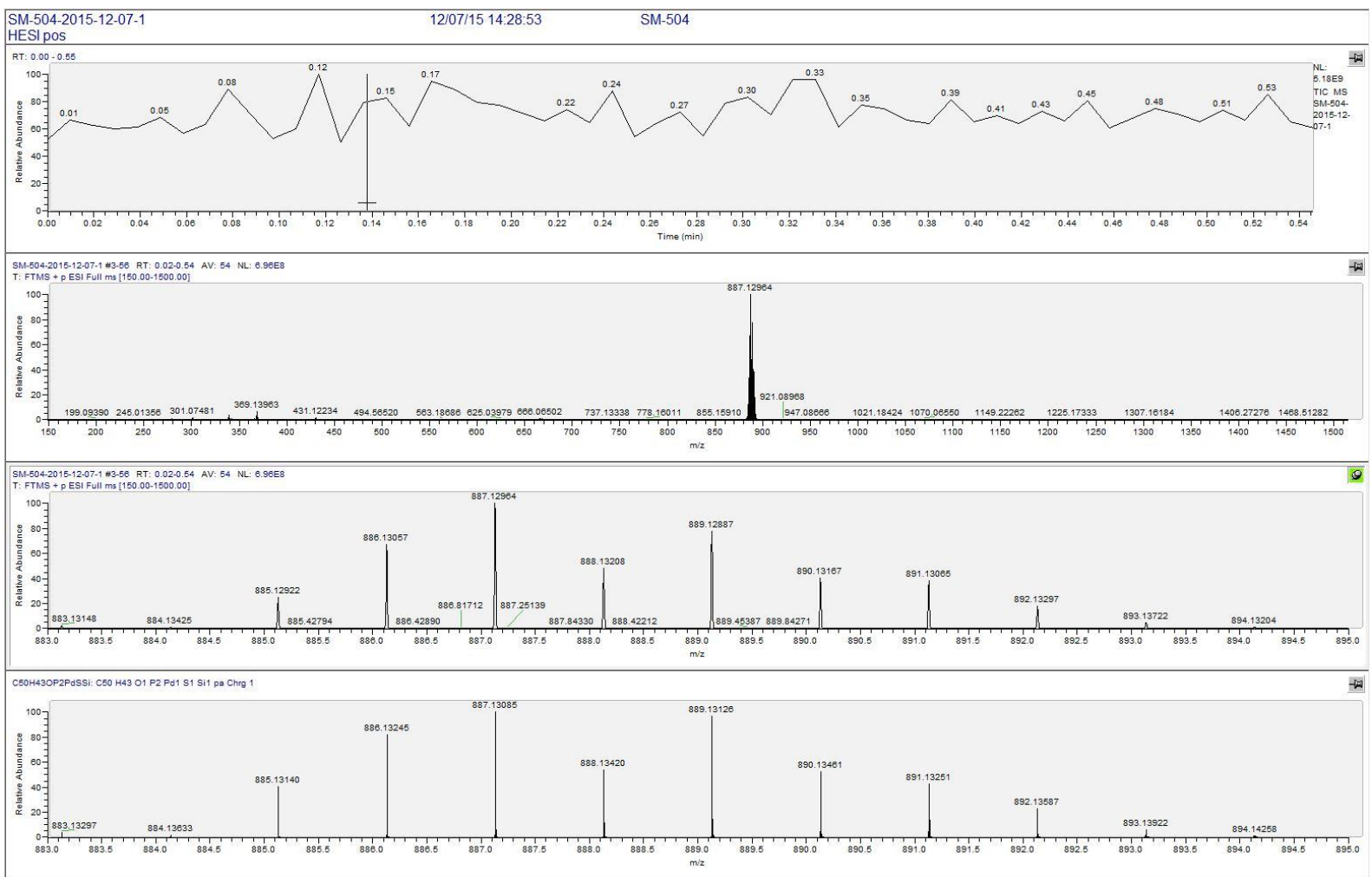

Figure S64. HRMS (ESI) spectrum of 2f. Calc. for $\mathrm{C}_{50} \mathrm{H}_{43} \mathrm{OP}_{2} \mathrm{PdSSi}\left([\mathrm{M}-\mathrm{Cl}]^{+}\right): \mathrm{m} / \mathrm{z}=887.13085 ; \mathrm{m} / \mathrm{z}=$ found: 887.12964; observed and calculated peak profile. 


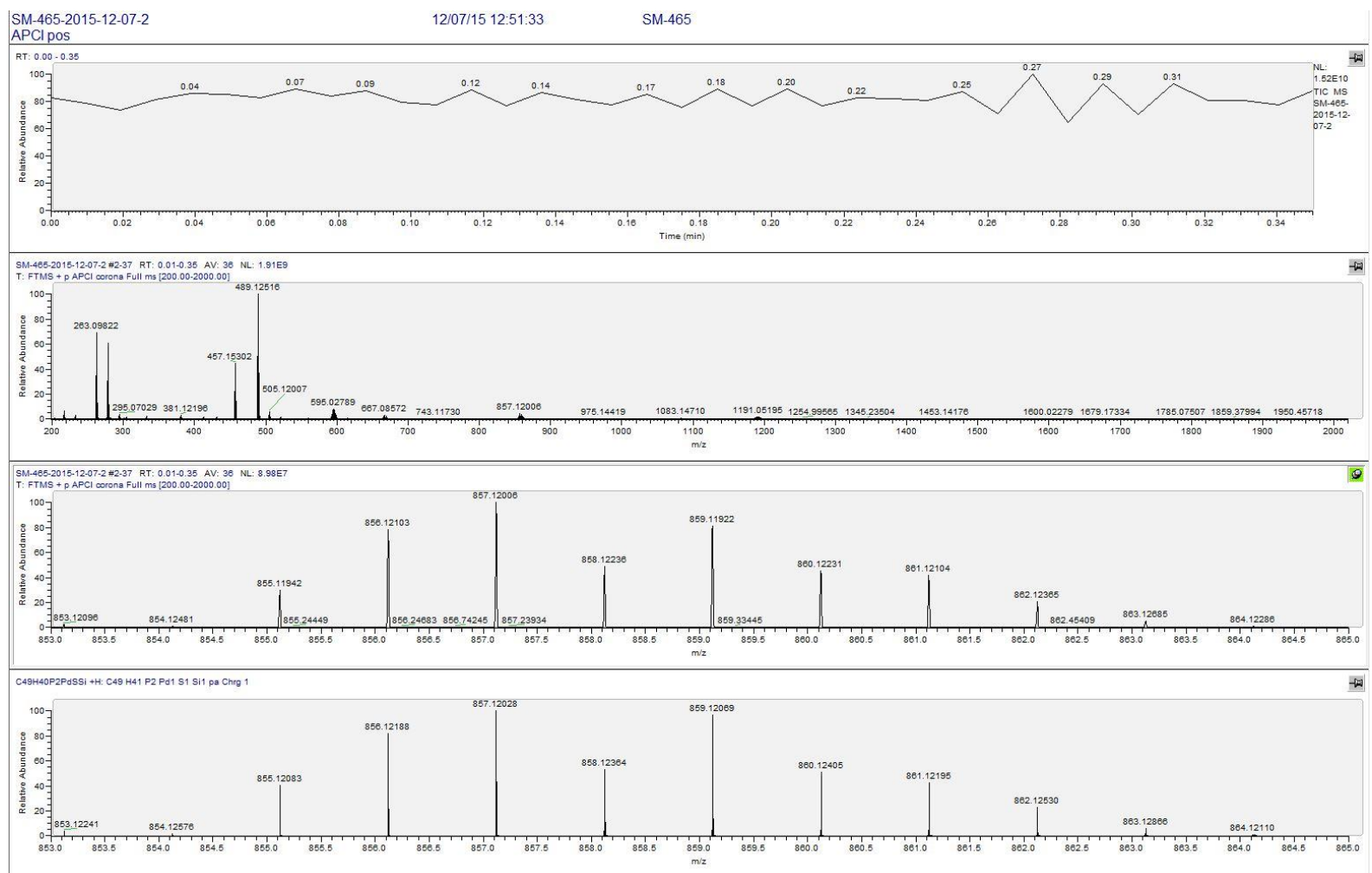

Figure S65. HRMS (APCI) spectrum of 3. Calc. for $\mathrm{C}_{49} \mathrm{H}_{41} \mathrm{P}_{2} \mathrm{PdSSi}\left([\mathrm{M}+\mathrm{H}]^{++}\right): \mathrm{m} / \mathrm{z}=857.12028$; found: $\mathrm{m} / \mathrm{z}=$ 857.12007, $595.02789\left(\left[\mathrm{M}+\mathrm{H}-\mathrm{PPh}_{3}\right]^{++}\right), 489.12517\left(\left[\mathrm{M}+\mathrm{H}-\mathrm{PdPPh}_{3}\right]^{+}\right), 457.15303\left(\left[\mathrm{M}+\mathrm{H}-\mathrm{SPdPPh}_{3}\right]^{++}\right)$; observed and calculated peak profile.

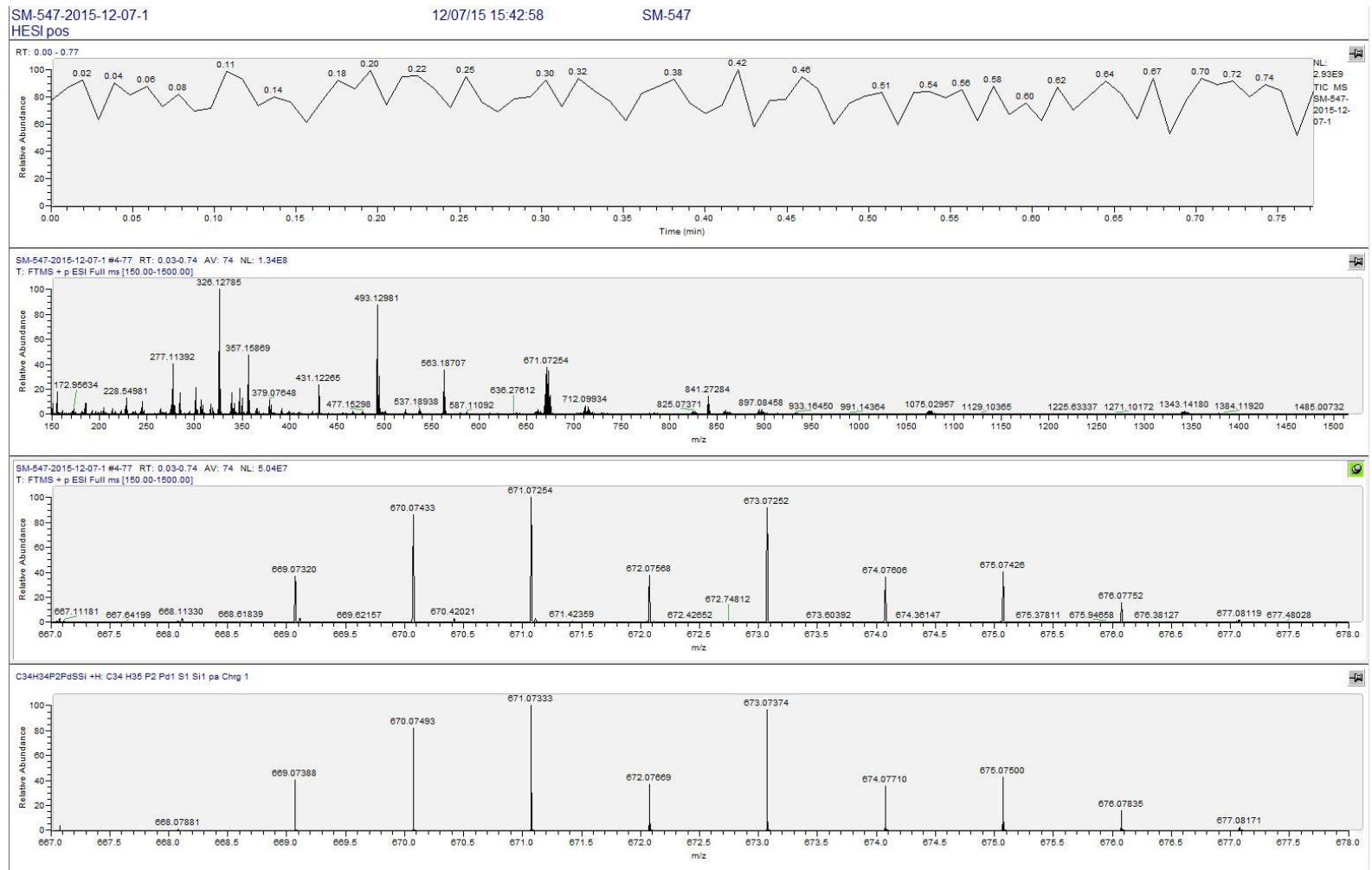

Figure S66. HRMS (ESI) spectrum of 5. Calc. for $\mathrm{C}_{34} \mathrm{H}_{35} \mathrm{P}_{2} \mathrm{PdSSi}\left([\mathrm{M}+\mathrm{H}]^{+\cdot}\right): \mathrm{m} / \mathrm{z}=671.07333$; found: $\mathrm{m} / \mathrm{z}=$ 671.07254; observed and calculated peak profile. 


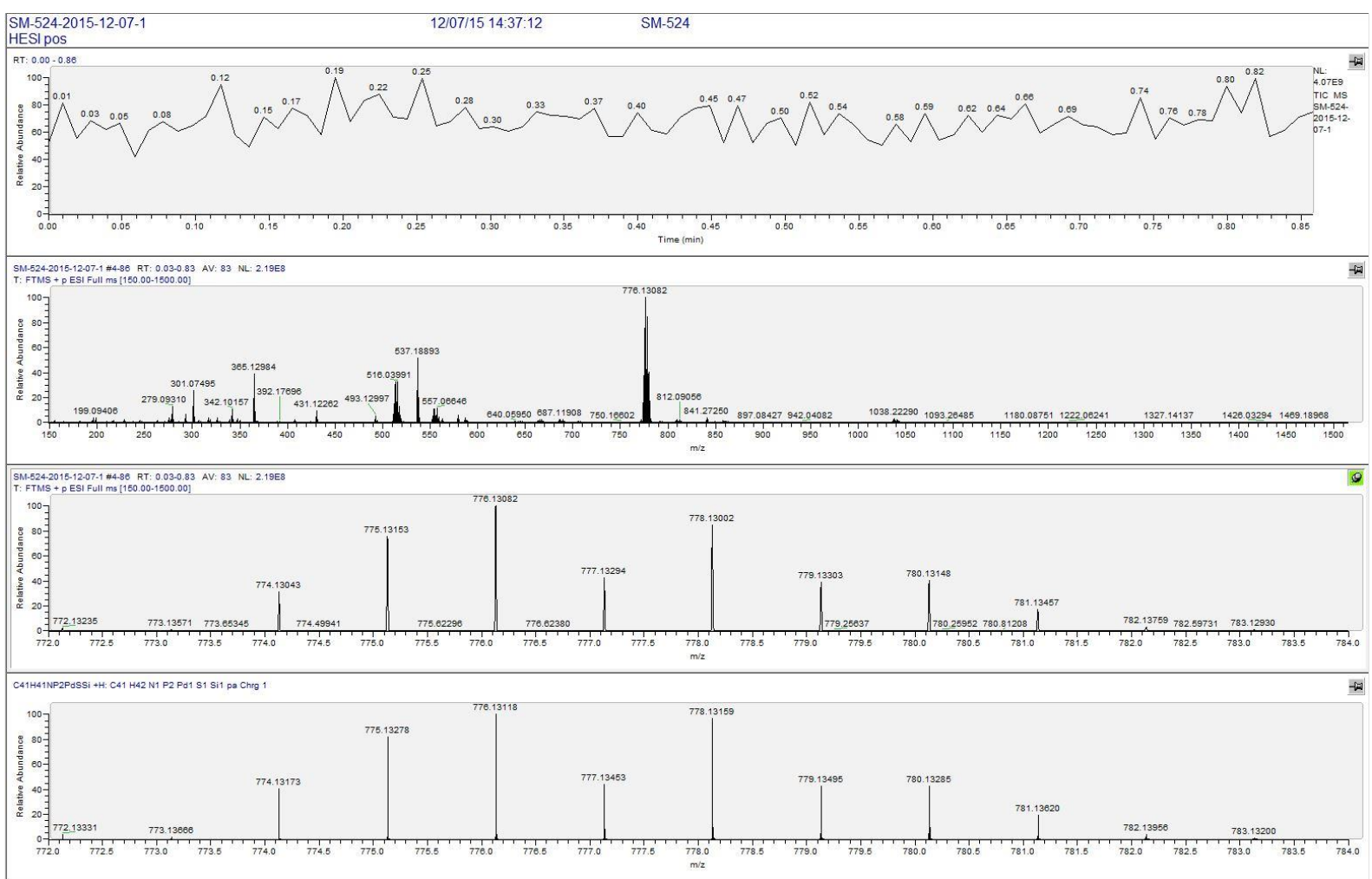

Figure S67. HRMS (ESI) spectrum of 6. Calc. for $\mathrm{C}_{41} \mathrm{H}_{42} \mathrm{NP}_{2} \mathrm{PdSSi}\left(\left[\mathrm{M}+\mathrm{H}+\mathrm{H}_{3} \mathrm{CCN}\right]^{+\cdot}\right): \mathrm{m} / \mathrm{z}=776.13118$; found: $\mathrm{m} / \mathrm{z}=776.13082$; observed and calculated peak profile.

\section{References}

[1] (a) Gessner, V. H. Organometallics 2011, 30, 4228. (b) Molitor, S.; Feichtner, K.-S.; Kupper, C.; Gessner, V. H. Chem. Eur. J. 2014, 20, 10752.

[2] G. M. Sheldrick, Acta Cryst. 2008, A64, 112-122. 